Innsbrucker Beiträge zur Fachdidaktik 8

Suzanne Kapelari (Hg.)

\title{
Vierte "Tagung der Fachdidaktik“ 2019 Interdisziplinäre fachdidaktische Diskurse zur Bildung für nachhaltige Entwicklung
}




\section{Innsbrucker Beiträge zur Fachdidaktik 8}

Series Editors: Andrea Brait, Barbara Hinger, Suzanne Kapelari 

Suzanne Kapelari (Hg.)

\section{Vierte „Tagung der Fachdidaktik“ 2019 Interdisziplinäre fachdidaktische Diskurse zur Bildung für nachhaltige Entwicklung}


Suzanne Kapelari

Institut für Fachdidaktik, Universität Innsbruck

Eine Veranstaltung der Universität Innsbruck und der Pädagogischen Hochschule Tirol

\section{pht}

PÄDAGOGISCHE
HOCHSCHULE TIROL

Review-Board:

Univ.-Prof. Dr. Thomas Hoffmann, Universität Innsbruck

Prof.in Dr.in Kerstin Kremer, Universität Hannover

Univ.-Prof.in Dr.in Anja Lembens, Universität Wien

Prof.in Dr.in Steffi Morkötter, Universität Rostock

Univ.-Prof. Dr. Thomas Rankin, Universität Linz

Univ.-Prof. Dr. Wolfgang Stadler, Universität Innsbruck

Sollte diese Publikation Links auf Webseiten Dritter enthalten, so übernehmen wir für deren Inhalt keine Haftung, da wir uns diese nicht zu eigen machen, sondern lediglich auf deren Stand zum Zeitpunkt der Erstveröffentlichung verweisen.

(C) innsbruck university press, 2020

Universität Innsbruck

1. Auflage

Alle Rechte vorbehalten.

www.uibk.ac.at/iup

ISBN 978-3-99106-019-2

DOI 10.15203/99106-019-2 


\section{Inhaltsverzeichnis}

Vorwort

S. Kapelari

\section{Plenarvortrag}

Bildung für nachhaltige Entwicklung -

Von Projekten zum Whole-Institution Approach

M. Rieckmann

\section{Vorträge}

Ein Donut, der alle satt macht?

Durch visuelle Datenanalyse mit GeoGebra und

Gapminder nachhaltige Entwicklung greifbar machen

M. Andre, A. Oberrauch, M. Zöttl

Vom Auswahlmodus zur Bewertungskompetenz

C. Wogowitsch

Transformative Bildung im naturwissenschaftlichen Unterricht

T. Kosler 
Eine interdisziplinäre Studie zum Einfluss von TBLT auf die Scientific Interlanguage von SchülerInnen im Biologie- und Physikunterricht

J. Taglieber, C. Pieber, S. Kapelari, W. Dür, B. Hinger

Literaturadaptionen in der Graphic Novel.

Eine weitere Axt für das gefrorene Meer in uns?

C. Bader

Implizite Bedeutung in sozialen Netzwerken Stärkung des gesellschaftlich-demokratischen Bewusstseins im Französisch als Fremdspracheunterricht

N. Brocca

Kulturelle Vielfalt in Österreichs Klassenzimmern: Welchen Beitrag kann eine Didaktik der Mehrkulturalität zur langfristig lernwirksamen Förderung von Lernenden im Englischunterricht der österreichischen Sekundarstufe I leisten? 206 J. Peskoller 


\section{Vorwort}

„Bildung für nachhaltige Entwicklung ist eines der zentralen Themen des 21. Jahrhunderts. Sie eröffnet allen Menschen die Chance zur Aneignung von Wissen, Werten und Kompetenzen um informierte Entscheidungen zu treffen und verantwortungsbewusst zum Schutz der Umwelt, für eine beständige Wirtschaft und einer gerechten Gesellschaft für aktuelle und zukünftige Generationen zu handeln und dabei die kulturelle Vielfalt zu respektieren" (Österreichische UNESCO-Kommission, 2020).

Im schulischen, außerschulischen und universitären Kontext ist nachhaltiges Denken und Handeln ein Bildungsziel, das nicht nur in den naturwissenschaftlichen oder technischen Unterrichtsfächern und Forschungsfeldern Niederschlag finden sollte. Als Querschnittsthema, das alle Unterrichts- wie auch Studienfächer miteinander vereint, sind alle Fachdidaktiken aufgerufen ihren Beitrag zur Bildung für eine nachhaltige Entwicklung zu leisten.

Es ist Aufgabe aller Fächer, die Lernenden beim Erwerb von Fähigkeiten und Kenntnissen zu unterstützen, die ihnen helfen die Qualität von Informationen einzuschätzen, ihre eigenen Entscheidungen zu prüfen und jenes Rüstzeug zur erwerben, das nachhaltiges Handeln ermöglicht.

Die globale Gesellschaft steht vor zahlreichen Herausforderungen, die das Individuum genauso wie das soziale Zusammenleben verschiedener Kulturen und Religionen, regionale, nationale und globale Wirtschaftssysteme und nicht zuletzt die Wissenschaft betreffen. Die vierte interdisziplinäre Tagung für Fachdidaktik 2019 verfolgte das Ziel, den interdisziplinären, fachdidaktischen Diskurs zu Fragen im Kontext der nachhaltigen Bildung nicht nur an der Pädagogischen Hochschule Tirol und der Fakultät für LehrerInnenbildung, sondern auch mit anderen Fakultäten an der Universität Innsbruck zu eröffnen. Der vorliegende Band umfasst eine Auswahl an Beiträgen dieser Tagung und zeigt, wie multiperspektivisch Bildung für eine Nachhaltige Entwicklung (BNE) betrachtet und wie die Auseinandersetzung mit ihren Zielen erfolgen kann. Innovation, Kreativität und Heterogenität, die nachhaltiges Denken erst

Kapelari, Suzanne (Hg.), Vierte „Tagung der Fachdidaktik“ 2019:

„Interdisziplinäre fachdidaktische Diskurse zur Bildung für nachhaltige Entwicklung“

(C) 2020 innsbruck university press, ISBN 978-3-99106-019-2, DOI 10.15203/99106-019-2 
ermöglichen, werden nicht nur in den thematischen Zugängen, sondern auch in den Forschungsmethoden sichtbar.

Einen Einstieg in das Thema bietet der Plenarvortrag von Professor Dr. Marco Rieckmann (Universität Vechta, Deutschland), der den Rahmen für die Tagung im Kontext der Hochschulentwicklung absteckt. ,Bildung für nachhaltige Entwicklung - Von Projekten zum Whole-Institution Approach' zeigte Möglichkeiten auf, wie die von den Vereinten Nationen verabschiedeten Nachhaltigen Entwicklungsziele (Sustainable Development Goals, SDGs) interpretiert und der Whole-Institution Approach als eine Leitlinie für eine umfassende Integration von BNE für eine nachhaltige Transformation von Schulen, Hochschulen und Bildungssystemen genützt werden kann.

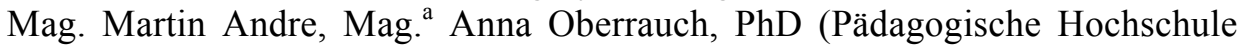
Tirol) und Melanie Zöttl (Universität Innsbruck) beschreiben eine interdisziplinäre Zusammenarbeit von Schule, Hochschule und Weiterbildung. ,Ein Donut, der alle satt macht? Durch visuelle Datenanalyse mit GeoGebra und Gapminder nachhaltige Entwicklung greifbar machen' gibt Einblick in eine interdisziplinäre Forschungs- und Praxisarbeit. Die Didaktik der Mathematik sowie der Geographie und Wirtschaftskunde schaffen die theoretischen Grundlagen zur Entwicklung und Evaluation einer fächerübergreifenden Lernumgebung.

Mag. ${ }^{a}$ Christine Wogowitsch (Universität Innsbruck), geht in ihrem Beitrag ,Vom Auswahlmodus zur Bewertungskompetenz' der Frage nach, wie Lernende zur Verantwortungsübernahme und zur Entwicklung von Werthaltungen im Sinne einer emanzipatorischen BNE angeleitet werden können.

Hochschulprofessor Dr. Thorsten Kosler, (Pädagogische Hochschule Tirol), analysiert in seinem Betrag $\mathrm{zu}$,Transformative Bildung im naturwissenschaftlichen Unterricht ${ }^{\star}$ drei Theorieansätze zur transformativen Bildung und beschreibt auf Basis dieser Analyse beispielhaft eine Möglichkeit, wie individuelles Lernen im naturwissenschaftlichen Unterricht gestaltet werden kann, damit dieser einen Beitrag $\mathrm{zu}$ einer gesellschaftlichen Transformation im Kontext einer nachhaltigen Entwicklung leisten kann.

Mag. ${ }^{a}$ Johanna Taglieber und Mag. ${ }^{a}$ Corinna Pieber (Universität Innsbruck) und weitere AutorInnen geben Einblick in ,Eine interdisziplinäre Studie zum Einfluss von TBLT auf die Scientific Interlanguage von SchülerInnen im Biologie- und Physikunterricht ${ }^{6}$. Eine Methode der Sprachdidaktik wird in den

Kapelari, Suzanne (Hg.), Vierte „Tagung der Fachdidaktik“ 2019:

„Interdisziplinäre fachdidaktische Diskurse zur Bildung für nachhaltige Entwicklung“

(C) 2020 innsbruck university press, ISBN 978-3-99106-019-2, DOI 10.15203/99106-019-2 
naturwissenschaftlichen Unterricht integriert, um die bildungssprachlichen Kompetenzen von Schülern und Schülerinnen zu fördern.

Literatur als Medium, das das Potential hat Menschen tief in ihrem Inneren zu berühren, beschreibt Mag. ${ }^{a}$ Caroline Bader (Universität Innsbruck) in ihrem Beitrag ,Literaturadaptionen in der Graphic Novel. Eine weitere Axt für das gefrorene Meer in uns?'. Für die Autorin kann Literaturunterricht auf einer tiefergreifenden Ebene für Nachhaltigkeit sorgen, indem die außerschulische literarische Realität in den Unterricht integriert wird, um so ein nachhaltiges literarisches Lernen zu ermöglichen.

Dr. Nicola Brocca (Universität Innsbruck) beschäftigt sich mit der ,Impliziten Bedeutung in sozialen Netzwerken - Stärkung des gesellschaftlichdemokratischen Bewusstseins im Französisch als Fremdspracheunterricht ${ }^{\star}$ und zeigt, welchen Beitrag die Sprachwissenschaft und die Fremdsprachendidaktik leisten können, um Ziele in der Medienbildung $\mathrm{zu}$ verfolgen, die eine demokratische und integrative Gesellschaft fördern.

Abschließend beschreibt Mag. ${ }^{a}$ Jasmin Peskoller (Universität Innsbruck) die ,Kulturelle Vielfalt in Österreichs Klassenzimmern' und stellt sich die Frage ,Welchen Beitrag kann eine Didaktik der Mehrkulturalität zur langfristig lernwirksamen Förderung von Lernenden im Englischunterricht der österreichischen Sekundarstufe I leisten'.

,Bildung für nachhaltige Entwicklung hat eine wichtige Bedeutung für die Förderung einer nachhaltigen Entwicklung der Gesellschaft. Sie ermöglicht Lernenden, nachhaltigkeitsrelevante Kompetenzen $\mathrm{zu}$ entwickeln und sich kritisch mit gesellschaftlichen Werten auseinanderzusetzen. Viele Bildungseinrichtungen widmen sich bereits einer Bildung für nachhaltige Entwicklung, oft aber eher noch in Projekten. Der Whole-Institution Approach kann als eine Leitlinie für eine umfassende Integration von BNE dienen und muss mit einem umfassenden Nachhaltigkeitsprozess einhergehen. (Rieckmann, in diesem Band).

Für eine tatsächliche Transformation der beteiligten Bildungseinrichtungen sind noch viele Schritte zu gehen. Die Ziele, die die Agenda einer Nachhaltigen Entwicklung der Vereinten Nationen verfolgt, werden aber in absehbarer Zukunft nicht an Bedeutung verlieren. Im Rahmen der, Vierten Tagung der Fachdidaktik' der Universität Innsbruck und der Pädagogischen Hochschule Tirol wurden die ersten Schritte unternommen, einen interdisziplinären, wissenschaftlichen Diskurs $\mathrm{zu}$ Themen der Bildung für eine Nachhaltige

Kapelari, Suzanne (Hg.), Vierte „Tagung der Fachdidaktik“ 2019:

„Interdisziplinäre fachdidaktische Diskurse zur Bildung für nachhaltige Entwicklung“

(C) 2020 innsbruck university press, ISBN 978-3-99106-019-2, DOI 10.15203/99106-019-2 
Entwicklung über Hochschulen, Fakultäts- und Fachgrenzen hinweg zu führen. Es liegt nun an allen beteiligten Akteuren diesen Diskurs weiterzuführen. Gedankt sei an dieser Stelle den Sponsoren der Tagung - dem Land Tirol, der Pädagogischen Hochschule Tirol, dem Regional Educational Competence Centre Mathematik \& Geometrie, dem Dekan der Fakultät für LehrerInnenbildung sowie der Vizerektorin für Forschung der Universität Innsbruck. Für die Unterstützung bei der Edition des Tagungsbands gilt der Dank insbesondere Julia Nagy, Susanne Rafolt, Konstantin Sagmeister, Thomas Schnabl und Johanna Taglieber, alle Institut für Fachdidaktik. Gedankt sei auch dem Team der ,,innsbruck university press“ für die gute Zusammenarbeit.

Innsbruck, im Juli 2020 


\title{
Bildung für nachhaltige Entwicklung - Von Projekten zum Whole-Institution Approach
}

\author{
Prof. Dr. Marco Rieckmann, Universität Vechta
}

\section{Einführung}

Der Globale Erdüberlastungstag 2019 (Earth Overshoot Day) war am 29. Juli. An diesem Tag waren die gesamten nachhaltig nutzbaren Ressourcen der Erde für das Jahr 2019 verbraucht, die der Weltbevölkerung rechnerisch zur Verfügung stünden. Jedes Jahr rückt dieses Datum im Kalender weiter nach vorn; die ökologische Krise auf dem Planeten spitzt sich also weiterhin zu. Die anthropogenen Treibhausgasemissionen steigen weltweit immer noch; ein großes Artensterben findet statt, das die Integrität des Planeten und die Fähigkeit der Erde gefährdet, die Bedürfnisse des Menschen zu befriedigen (UN Environment, 2019).

Vor diesem Hintergrund wird die Dringlichkeit einer nachhaltigen Entwicklung deutlich. Wesentliche Elemente einer nachhaltigen Entwicklung sind (Heinrichs, 2007; Kopfmüller et al., 2001; Michelsen, 2007; Ott \& Döring, 2004):

- Gerechtigkeit: Eine nachhaltige Entwicklung strebt nach intragenerationeller Gerechtigkeit (Verteilungsgerechtigkeit zwischen Nord und Süd, Reich und Arm) und intergenerationeller Gerechtigkeit (Ausgleich zwischen heutigen und künftigen Generationen). 
- Ökologische Grenzen: Alle unsere Aktivitäten und Güter haben natürliche Grundlagen (Retinität ${ }^{1}$ ). Die ökologische Tragfähigkeit beschreibt die Grenzen unserer wirtschaftlichen Aktivitäten und unserer gesellschaftlichen Entwicklung.

- Globale Orientierung: Die Analyse von Problemen der Nicht-Nachhaltigkeit und deren Lösung verlangen nach einer globalen Orientierung.

- Partizipation: Eine nachhaltige Entwicklung ist ein gesellschaftlicher Lern-, Verständigungs- und Gestaltungsprozess, der erst durch die Beteiligung möglichst Vieler mit Ideen und Visionen gefüllt und vorangetrieben werden kann.

Gerade mit Bezug auf den letzten Punkt der Partizipation ist es eine gute Nachricht, dass insbesondere junge Menschen sich der ökologischen Krise zunehmend bewusst sind und u.a. im Kontext der ,Fridays for Future'Bewegung eine grundlegende Transformation vehement einfordern.

Leitlinien für diese notwendige, globale Transformation zeigen die Sustainable Development Goals (SDGs) auf. Diese 17 Nachhaltigkeitsziele bilden den Kern der am 25. September 2015 von der UN-Vollversammlung verabschiedeten Agenda 2030 für Nachhaltige Entwicklung (Vereinte Nationen, 2015). Dieses neue globale Rahmenwerk zur nachhaltigen Entwicklung der Weltgesellschaft wurde im Anschluss an die UN-Konferenz über nachhaltige Entwicklung $($ Rio+20) in Rio de Janeiro (Brasilien) im Juni 2012 in einem dreijährigen Prozess entwickelt, an dem neben den UN-Mitgliedstaaten, NGOs und anderen Akteuren auch Millionen von Menschen aus der ganzen Welt beteiligt waren (Kercher, 2015; Scholz, 2015, 2017).

Die universalen, transformativen und inklusiven SDGs beschreiben wichtige Entwicklungsherausforderungen für die Menschheit. Das Ziel der 17 SDGs ist es, für alle, jetzt und in Zukunft, ein nachhaltiges, friedliches, wohlhabendes und gerechtes Leben zu ermöglichen. Die SDGs beziehen sich auf globale Herausforderungen, die für das Überleben der Menschheit entscheidend sind. Sie setzen ökologische Grenzen fest und definieren kritische Schwellenwerte

1 Der Rat von Sachverständigen für Umweltfragen (SRU) hebt in seinem Umweltgutachten 1994 das Schlüsselprinzip ,Retinität' hervor, womit die Gesamtvernetzung aller menschlichen Tätigkeiten und Erzeugnisse mit der sie tragenden Natur gemeint ist (Rat von Sachverständigen für Umweltfragen [SRU], 1994).

Kapelari, Suzanne (Hg.), Vierte „Tagung der Fachdidaktik“ 2019:

„Interdisziplinäre fachdidaktische Diskurse zur Bildung für nachhaltige Entwicklung“

(C) 2020 innsbruck university press, ISBN 978-3-99106-019-2, DOI 10.15203/99106-019-2 
für den Einsatz natürlicher Ressourcen. Sie erkennen an, dass Armutsbekämpfung Hand in Hand gehen muss mit Strategien, die die wirtschaftliche Entwicklung befördern. Sie widmen sich einer Reihe von sozialen Bedürfnissen, einschließlich Bildung, Gesundheit, sozialer Absicherung und Beschäftigungsmöglichkeiten, während sie gleichzeitig auf die Bewältigung des Klimawandels und Umweltschutz abzielen. Die SDGs adressieren die wichtigsten systemischen Barrieren für eine nachhaltige Entwicklung wie Ungleichheit, nicht-nachhaltige Konsummuster, schwache institutionelle Kapazitäten und Umweltzerstörung (Messner \& Scholz, 2015; Scholz, 2015, 2017).

Ein wesentliches Merkmal der Agenda 2030 für Nachhaltige Entwicklung ist ihre Universalität und Unteilbarkeit. Sie richtet sich an alle Länder - aus dem Globalen Süden und dem Globalen Norden. Alle Länder, die sich der Agenda 2030 verschreiben, müssen ihre eigenen Entwicklungsbemühungen an dem Ziel ausrichten, den Wohlstand zu fördern und gleichzeitig den Planeten $\mathrm{zu}$ schützen, um eine nachhaltige Entwicklung zu erreichen (Messner \& Scholz, 2015). Insofern können in Bezug auf die SDGs alle Länder als Entwicklungsländer betrachtet werden, und alle Länder müssen dringende Maßnahmen zur Förderung einer nachhaltigen Entwicklung ergreifen.

Seit Ende der 1990er Jahre wird in der bildungswissenschaftlichen Diskussion sowie der Bildungspraxis verstärkt Bezug auf eine nachhaltige Entwicklung genommen. In diesem Kontext ist das Konzept einer Bildung für nachhaltige Entwicklung (BNE) entwickelt worden. Bildung für nachhaltige Entwicklung zielt darauf ab, Menschen zu befähigen, sich an den gesellschaftlichen Lernund Verständigungsprozessen für eine nachhaltige Entwicklung, der Umsetzung der SDGs und damit der Förderung einer "Großen Transformation“ (Wissenschaftlicher Beirat der Bundesregierung Globale Umweltveränderungen [WBGU], 2011) zu beteiligen.

Die UN-Dekade ,Bildung für nachhaltige Entwicklung' hat mit dem innovativen Konzept einer BNE dazu beigetragen, dass Bildung in den unterschiedlichen Bildungsbereichen eine neue Bedeutung bekommen hat. Die Aktivitäten der UN-Dekade wurden seit 2015 im Weltaktionsprogramm (WAP) ,Bildung für nachhaltige Entwicklung، (2015-2019) fortgeführt. Dessen Ziel war es, BNE in alle Bildungsbereiche zu integrieren, um damit eine nachhaltige

Kapelari, Suzanne (Hg.), Vierte „Tagung der Fachdidaktik“ 2019:

„Interdisziplinäre fachdidaktische Diskurse zur Bildung für nachhaltige Entwicklung“

(C) 2020 innsbruck university press, ISBN 978-3-99106-019-2, DOI 10.15203/99106-019-2 
Entwicklung der Weltgesellschaft zu fördern. ${ }^{2}$ Denn bisher ist BNE in den verschiedenen Bildungsbereichen in sehr unterschiedlichem Ausmaß verankert worden (Rieckmann, 2016).

Während aktuell die Bildungslandschaft immer noch eher durch einzelne BNEProjekte gekennzeichnet ist, geht es nun um die Verankerung in den Strukturen. Eines der fünf Handlungsfelder des WAP war daher die ganzheitliche Transformation von Lern- und Lehrumgebungen. Bildungseinrichtungen sollten sich als Orte des Lernens und der Erfahrung für eine nachhaltige Entwicklung verstehen und sollten daher alle ihre Prozesse an Prinzipien der Nachhaltigkeit ausrichten. Damit BNE wirksamer ist, muss jede Bildungseinrichtung als Ganzes verändert werden. Ein solches ganzheitliches Konzept (WholeInstitution Approach) zielt darauf ab, Nachhaltigkeit in alle Aspekte der jeweiligen Bildungseinrichtung zu integrieren. Es geht darum, das Curriculum, den Betrieb, die Organisationskultur, die Beteiligung der Lernenden, die Leitung und das Management, die Beziehungen zur Kommune und die Forschung neu zu denken. Auf diese Weise fungiert die Institution selbst als Vorbild für die Lernenden (United Nations Educational, Scientific and Cultural Organization [UNESCO], 2014).

Dieser Beitrag gibt zunächst einen Überblick über das Konzept der Bildung für nachhaltige Entwicklung. Anschließend wird auf die Rolle von BNE für das Erreichen der SDGs eingegangen, und es wird der ganzheitliche BNE-Ansatz (Whole-Institution Approach) dargestellt. Am Beispiel der Universität Vechta wird abschließend veranschaulicht, wie der Whole-Institution Approach im Rahmen eines universitären Nachhaltigkeitsprozesses Anwendung finden kann.

2 Mit Beginn des Jahres 2020 hat die UNESCO das neue Programm, Education for Sustainable Development: Towards achieving the SDGs ${ }^{\star}$ - kurz ,ESD for $2030^{`}$ - aufgelegt.

Kapelari, Suzanne (Hg.), Vierte „Tagung der Fachdidaktik“ 2019:

„Interdisziplinäre fachdidaktische Diskurse zur Bildung für nachhaltige Entwicklung“

(C) 2020 innsbruck university press, ISBN 978-3-99106-019-2, DOI 10.15203/99106-019-2 


\section{Das Konzept einer Bildung für nachhaltige Entwicklung}

\subsection{Unterschiedliche Ansätze einer Bildung für nachhaltige Entwicklung}

Ohne Lernprozesse wird eine nachhaltige Entwicklung nicht möglich sein (Vare \& Scott, 2007). Daher soll Bildung für nachhaltige Entwicklung dazu beitragen, eine nachhaltige Entwicklung der Gesellschaft zu fördern. Wie Bildung für nachhaltige Entwicklung dies aber am besten erreichen kann, dazu gibt es im diesbezüglichen Diskurs unterschiedliche Auffassungen. Vare \& Scott (2007) unterscheiden zwei Strömungen der Bildung für nachhaltige Entwicklung (Education for Sustainable Development - ESD): ESD 1 und ESD 2. Diese Unterscheidung findet sich auch bei Wals (2011), der von einem ,instrumental approach“ und einem „emancipatory approach“ spricht.

ESD 1 geht von der Überzeugung aus, dass es bestimmte Werte und Verhaltensweisen gibt, die eindeutig mit einer nachhaltigen Entwicklung verbunden sind. ExpertInnen können diese identifizieren. Ziel von BNE ist es dementsprechend, Bewusstsein für eine nachhaltige Entwicklung zu schaffen, mit Nachhaltigkeit verbundene Werte zu vermitteln und als nachhaltig erkannte Verhaltensweisen zu fördern. Es sollen durch die Bildungsprozesse bestimmte Verhaltensweisen wie die Trennung von Müll oder das Sparen von Energie befördert werden (Vare \& Scott, 2007). Unter Bezug auf Ansätze der Umweltpsychologie (Wals, 2011) werden z.B. Anreize gegeben, um ein bestimmtes, vermeintlich nachhaltiges Verhalten attraktiv zu machen.

Dieser Ansatz einer Bildung für nachhaltige Entwicklung im Sinne von ESD 1 wird z.B. in den Zielsetzungen der UN-Dekade, Bildung für nachhaltige Entwicklung' (2005-2014) und des nachfolgenden Weltaktionsprogramms (WAP) deutlich:

„Der [sic] WAP trägt dazu bei, jene Vision zu erreichen, für die sich bereits die UN-Dekade eingesetzt hat: ,eine Welt, in der alle von Bildung profitieren können und die Werte, Verhaltensweisen und Lebensstile erlernen, die für eine nachhaltige Zukunft und für eine positive gesellschaftliche Transformation nötig sind"." (UNESCO, 2014)

Kapelari, Suzanne (Hg.), Vierte ,Tagung der Fachdidaktik“ 2019:

„Interdisziplinäre fachdidaktische Diskurse zur Bildung für nachhaltige Entwicklung“

(C) 2020 innsbruck university press, ISBN 978-3-99106-019-2, DOI 10.15203/99106-019-2 
Aber auch in Stellungnahmen von Regierungen und Nicht-Regierungsorganisationen wird häufig Bezug darauf genommen, dass von einer Bildung für nachhaltige Entwicklung direkte Beiträge zu einer nachhaltigeren Lebensweise erwartet werden.

ESD 2 hingegen geht von einem reflexiveren Ansatz aus. Hier geht es weniger darum, Denk- oder Verhaltensweisen vorzugeben, sondern Individuen sollen in die Lage versetzt werden, selbst über Fragen einer nachhaltigen Entwicklung nachzudenken und ihre eigenen Antworten zu finden (Vare \& Scott, 2007). In diesem Zusammenhang wird nachhaltige Entwicklung nicht als ein geschlossener Experten-Diskurs, sondern als ein offener gesellschaftlicher (Lern-) Prozess gesehen. Leitend ist dabei die Erkenntnis, dass oft gar nicht sicher ist, welche Verhaltensweisen effektiv die nachhaltigeren sind (Wals, 2011).

„In ESD 2, we can't measure success in terms of environmental impacts because this is an open-ended process; outcomes will depend on people's unforeseen decisions in future, unforeseeable circumstances. But we can research the extent to which people have been informed and motivated, and been enabled to think critically and feel empowered to take responsibility." (Vare \& Scott, 2007)

Bildung für nachhaltige Entwicklung im Sinne von ESD 2 befähigt zu einer kritischen Auseinandersetzung mit einer nachhaltigen Entwicklung und mit der Komplexität, der Unsicherheit sowie den Widersprüchen, die mit ihr verbunden sind. Hier wird eine nachhaltige Entwicklung selbst als ein Lernprozess verstanden (Vare \& Scott, 2007). Im Fokus stehen Kompetenzentwicklung und kritisches Denken als Voraussetzungen für das Treffen von eigenen Entscheidungen im Sinne einer nachhaltigen Entwicklung (Mulder, 2014; Wals, 2011). Tabelle 1 gibt einen Überblick über die beiden Strömungen. 
Tab. 1: ESD 1 und ESD 2 (Vare \& Scott, 2007, S. 193f.)

\begin{tabular}{|l|ll|}
\hline ESD 1 & $\bullet$ & Promoting/facilitating changes in what we do \\
\cline { 2 - 3 } & $\begin{array}{l}\text { - } \\
\text { Promoting (informed, skilled) behaviours and ways of thinking, } \\
\text { where the need for this is clearly identified and agreed }\end{array}$ \\
\cline { 2 - 3 } & $\bullet$ & Learning for sustainable development \\
\hline
\end{tabular}

\begin{tabular}{|l|ll|}
\hline ESD 2 & $\begin{array}{l}\text { Building capacity to think critically about (and beyond) what } \\
\text { experts say and to test sustainable development ideas }\end{array}$ \\
\cline { 2 - 3 } & $\bullet$ & Exploring the contradictions inherent in sustainable living \\
\cline { 2 - 3 } & $\bullet$ & Learning as sustainable development \\
\hline
\end{tabular}

Auch wenn Vare \& Scott (2007) beide Ansätze als komplementär betrachten, favorisieren sie aus pädagogischer Perspektive doch ESD 2 - so wie Wals (2011) sich für den von ihm als ,emancipatory approach“ bezeichneten Ansatz ausspricht.

Diese Argumentation findet sich auch bei de Haan: Bildung für nachhaltige Entwicklung beziehe sich nicht auf die Ziele, Zwecke und Absichten des Handelns von Individuen, sondern auf die Wirkungen. Sie soll Individuen in die Lage versetzen, ,wenn sie entsprechende Ziele, Zwecke oder Absichten haben“, im Sinne einer nachhaltigen Entwicklung handeln zu können (de Haan et al., 2008, S. 117). Es geht mithin um die „Eröffnung von Möglichkeiten“ (de Haan et al., 2008, S. 123) und nicht darum, zu einem bestimmten vermeintlich nachhaltigkeitskonformen Verhalten $\mathrm{zu}$ erziehen. Lernende sollen - als „NachhaltigkeitsbürgerInnen“ (Rieckmann \& Schank, 2016; Wals, 2015; Wals \& Lenglet, 2016) - befähigt werden, „eine nachhaltige Entwicklung mitzugestalten und ihre eigenen Handlungen diesbezüglich kritisch zu reflektieren“ (Künzli David, 2007, S. 35).

Dieser emanzipatorische Ansatz einer Bildung für nachhaltige Entwicklung sieht als das wesentliche Bildungsziel die Entwicklung von Schlüsselkompetenzen an, die Individuen $\mathrm{zu}$ einer aktiven Gestaltung des Prozesses einer nachhaltigen Entwicklung befähigen. 


\subsection{Kompetenzorientierung einer Bildung für nachhaltige Entwicklung}

Im Diskurs über eine Bildung für nachhaltige Entwicklung wird seit einigen Jahren intensiv die Frage diskutiert, über welche Schlüsselkompetenzen ${ }^{3}$ Individuen verfügen sollten, um ihr eigenes Leben sowie das gesellschaftliche Umfeld aktiv im Sinne einer nachhaltigen Entwicklung mitgestalten zu können (Lozano, Merrill, Sammalisto, Ceulemans, \& Lozano, 2017; Overwien, 2013; Rieckmann, 2013; 2018; Wiek, Withycombe, \& Redman, 2011). Dabei wird in Deutschland häufig Bezug auf das Konzept der Gestaltungskompetenz genommen: „Gestaltungskompetenz bezeichnet die Fähigkeit, Probleme nicht nachhaltiger Entwicklungen erkennen und Wissen über nachhaltige Entwicklung wirksam anwenden zu können“ (de Haan, 2008, S. 12). Sie umfasst zwölf Teilkompetenzen (ebd.):

- Kompetenz zur Perspektivübernahme,

- Kompetenz zur Antizipation,

- Kompetenz zur Disziplinen übergreifenden Erkenntnisgewinnung,

- Kompetenz zum Umgang mit unvollständigen und überkomplexen Informationen,

- Kompetenz zur Kooperation,

- Kompetenz zur Bewältigung individueller Entscheidungsdilemmata,

- Kompetenz zur Partizipation,

- Kompetenz zur Motivation,

- Kompetenz zur Reflexion auf Leitbilder,

- Kompetenz zum moralischen Handeln,

- Kompetenz zum eigenständigen Handeln,

- Kompetenz zur Unterstützung anderer.

Das Konzept der Gestaltungskompetenz zeichnet sich also durch Kompetenzen aus, „die eine zukunftsweisende und eigenverantwortliche Mitgestaltung einer

3 Kompetenzen sind individuelle Selbstorganisationsdispositionen (Erpenbeck \& Heyse, 2007), die sich aus Wissen, Fähigkeiten und Fertigkeiten, Motiven und emotionalen Elementen zusammensetzen (Heil, 2007; Klieme \& Hartig, 2007; Weinert, 2001). Schlüsselkompetenzen sind transversale, multifunktionale und kontextübergreifende Kompetenzen, die zentral für die Erreichung wichtiger gesellschaftlicher Ziele und daher für alle Individuen von Bedeutung sind (Chur, 2012; Michelsen \& Rieckmann, 2014; Rieckmann, 2013; Rychen, 2003; Weinert, 2001).

Kapelari, Suzanne (Hg.), Vierte „Tagung der Fachdidaktik“ 2019:

„Interdisziplinäre fachdidaktische Diskurse zur Bildung für nachhaltige Entwicklung“

(C) 2020 innsbruck university press, ISBN 978-3-99106-019-2, DOI 10.15203/99106-019-2 
nachhaltigen Entwicklung ermöglichen“ (Michelsen, 2009, S. 84). Ergänzend zur Gestaltungskompetenz werden auch das Konzept der Bewertungskompetenz (Bögeholz, 2007) und dasjenige der Transformative Literacy (Schneidewind, 2013) diskutiert und angewendet.

Zudem findet insbesondere im Globalen Lernen der, Orientierungsrahmen für den Lernbereich Globale Entwicklung im Rahmen einer Bildung für nachhaltige Entwicklung' (Kultusministerkonferenz [KMK] \& Bundesministerium für wirtschaftliche Zusammenarbeit und Entwicklung [BMZ], 2015) breite Anwendung. Er formuliert Kernkompetenzen des Lernbereichs Globale Entwicklung in den drei Bereichen Erkennen, Bewerten und Handeln (Tabelle 2).

Tab. 2: Kernkompetenzen des Lernbereichs Globale Entwicklung (KMK \& BMZ, 2015, S. 96f.)

\begin{tabular}{|c|c|c|}
\hline Erkennen & Bewerten & Handeln \\
\hline $\begin{array}{ll}\text { - } & \text { Informationsbeschaffung } \\
\text { - } & \text { End -verarbeitung } \\
\text { - } & \text { Analyse des Globalen } \\
& \text { Wandels } \\
\text { - } & \text { Unterscheidung von } \\
& \text { Handlungsebenen }\end{array}$ & $\begin{array}{ll}\text { - } & \text { Perspektivenwechsel } \\
\text { und Empathie } \\
\text { - } \\
\text { Kritische Reflexion } \\
\text { und Stellungnahme } \\
\text { - Beurteilen von } \\
\text { Entwicklungs- } \\
\text { maßnahmen }\end{array}$ & $\begin{array}{ll}\text { - } & \text { Solidarität und } \\
\text { Mitverantwortung } \\
\text { - } & \text { Verständigung und } \\
\text { Konfliktlösung } \\
\text { - } \\
\text { Handlungsfähigkeit im } \\
\text { globalen Wandel } \\
\text { - } \\
\text { Partizipation und } \\
\text { Mitgestaltung }\end{array}$ \\
\hline
\end{tabular}

Auf internationaler Ebene hat sich die OECD in ihrem Projekt, Definition and Selection of Competencies' (DeSeCo) damit befasst, grundlegende Kompetenzen für das Leben in einer interdisziplinären und internationalen Wissensgesellschaft $\mathrm{zu}$ formulieren (Rychen, 2003). Die Ergebnisse des DeSeCo-Projekts haben Eingang in den deutschen BNE-Diskurs gefunden, so dass Gestaltungskompetenz in Beziehung $\mathrm{zu}$ den DeSeCoSchlüsselkompetenzen gesetzt wird (de Haan, 2008). Zudem findet auch auf der internationalen Ebene eine explizite Auseinandersetzung mit der Kompetenzförderung durch Bildung für nachhaltige Entwicklung statt (Dlouhá, Heras, Mulà, Perez Salgado, \& Henderson, 2019; Lozano et al., 2017; Mogensen \& Schnack, 2010; Rieckmann, 2018; Thomas, Barth, \& Day, 2013; UNESCO, 2017; Wals, 2010; 
Wiek et al., 2011, 2016). In einer Delphi-Studie mit ExpertInnen für Bildung für nachhaltige Entwicklung aus Deutschland, England, Ecuador, Chile und Mexiko (Rieckmann, 2010, 2011) konnte gezeigt werden, dass die in den unterschiedlichen (nationalen) Diskursen diskutierten Schlüsselkompetenzen international vergleichbar sind. Länderübergreifend wurden die Kompetenz zum vernetzten Denken und Umgang mit Komplexität, die Kompetenz zum vorausschauenden Denken und die Kompetenz zum kritischen Denken als die wichtigsten Schlüsselkompetenzen identifiziert, die Individuen für das Verstehen zentraler Probleme der Weltgesellschaft und deren nachhaltigen Gestaltung benötigen.

Mit ihren Key Competencies in Sustainability führen Wiek et al. (2011, 2016) verschiedene international diskutierte Konzepte zusammen und unterscheiden mit einem besonderen Fokus auf nachhaltigkeitswissenschaftliche Studiengänge - fünf Schlüsselkompetenzen: Systems Thinking Competence, Anticipatory Competence, Normative Competence, Strategic Competence, Interpersonal Competence.

Neben diesen von Wiek et al. $(2011,2016)$ benannten Kompetenzen werden immer wieder auch die Kompetenz zum Kritischen Denken sowie Selbstkompetenz als besonders wichtig für eine nachhaltige Entwicklung angesehen, sodass die folgenden acht Nachhaltigkeitskompetenzen derzeit im internationalen BNE-Diskurs als besonders relevant angesehen werden (Rieckmann, 2018; UNESCO, 2017):

- Kompetenz zum Vernetzten Denken: die Fähigkeiten, Zusammenhänge zu erkennen und $\mathrm{zu}$ verstehen; komplexe Systeme $\mathrm{zu}$ analysieren; $\mathrm{zu}$ überlegen, wie Systeme in verschiedene Domänen und verschiedene Skalen eingebettet sind; und mit Unsicherheit umzugehen

- Kompetenz zum Vorausschauenden Denken: die Fähigkeiten, multiple (mögliche, wahrscheinliche und wünschenswerte) Zukünfte zu verstehen und $\mathrm{zu}$ bewerten; eigene Visionen für die Zukunft $\mathrm{zu}$ schaffen; das Vorsorgeprinzip anzuwenden; die Konsequenzen von Handlungen zu beurteilen; und mit Risiken und Veränderungen umzugehen

- Normative Kompetenz: die Fähigkeiten, die Normen und Werte zu verstehen und zu reflektieren, die den eigenen Handlungen zugrunde liegen; und Nachhaltigkeitswerte, Prinzipien und Ziele im Kontext von Interessenkonflikten und Trade-Offs, unsicheren Kenntnissen und Widersprüchen zu verhandeln

Kapelari, Suzanne (Hg.), Vierte „Tagung der Fachdidaktik“ 2019:

„Interdisziplinäre fachdidaktische Diskurse zur Bildung für nachhaltige Entwicklung“

(C) 2020 innsbruck university press, ISBN 978-3-99106-019-2, DOI 10.15203/99106-019-2 
- Strategische Kompetenz: die Fähigkeiten zur kollektiven Entwicklung und Umsetzung innovativer Maßnahmen, die Nachhaltigkeit auf lokaler Ebene und darüber hinaus voranbringen

- Kooperationskompetenz: die Fähigkeiten, von anderen zu lernen; die Bedürfnisse, Perspektiven und Handlungen anderer $\mathrm{zu}$ verstehen und $\mathrm{zu}$ respektieren (Empathie), andere $\mathrm{zu}$ verstehen, eine Beziehung $\mathrm{zu}$ ihnen aufzubauen und für sie empfindsam zu sein (empathische Führung); mit Konflikten in einer Gruppe umzugehen; und eine kollaborative und partizipative Problemlösung zu ermöglichen

- Kompetenz zum Kritischen Denken: die Fähigkeit, Normen, Praktiken und Meinungen $\mathrm{zu}$ hinterfragen; die eigenen Werte, Wahrnehmungen und Handlungen zu reflektieren; und sich im Nachhaltigkeitsdiskurs zu positionieren

- Selbstkompetenz: die Fähigkeit, über die eigene Rolle in der lokalen Gemeinschaft und (globalen) Gesellschaft nachzudenken; kontinuierlich seine Handlungen zu bewerten und sich weiter zu motivieren; und sich mit den eigenen Gefühlen und Wünschen auseinanderzusetzen

- Integrierte Problemlösekompetenz: die übergreifende Fähigkeit, unterschiedliche Problemlösungsrahmen für komplexe Nachhaltigkeitsprobleme anzuwenden und passfähige, inklusive und gerechte Lösungsmöglichkeiten $\mathrm{zu}$ entwickeln, die eine nachhaltige Entwicklung fördern und die oben genannten Kompetenzen integrieren

Es wird deutlich, dass die nachhaltigkeitsrelevanten Schlüsselkompetenzen aktuell diskutierte gesellschaftliche Handlungs- und Gestaltungsfelder widerspiegeln. Hiermit ist ein Bezug zu Klafkis Konzept der Allgemeinbildung und seinen epochaltypischen Schlüsselproblemen (Klafki, 2007) sowie Krügers Begriff der reflexiven Erziehungswissenschaft (Krüger, 2012) gegeben. Es geht darum, Lernenden eine Auseinandersetzung mit gesellschaftlichen Schlüsselthemen $\mathrm{zu}$ ermöglichen und sie damit auf heutige und zukünftige Problemlösungen vorzubereiten (Gräsel et al., 2012; Overwien, 2013). Der Erwerb von nachhaltigkeitsrelevanten Schlüsselkompetenzen soll Lernende befähigen, mit „prototypischen Handlungshemmnissen und -störungen“ (de Haan et al., 2008, S. 125) umzugehen, die bei der Förderung und Gestaltung einer nachhaltigen Entwicklung häufig auftreten. Dabei geht es vor allem um das Entscheiden und Handeln im Kontext individueller Entscheidungs-

Kapelari, Suzanne (Hg.), Vierte „Tagung der Fachdidaktik“ 2019:

„Interdisziplinäre fachdidaktische Diskurse zur Bildung für nachhaltige Entwicklung“

(C) 2020 innsbruck university press, ISBN 978-3-99106-019-2, DOI 10.15203/99106-019-2 
dilemmata, aber auch um Entscheiden und Handeln bei unvollständiger oder überkomplexer Information, in kollektiven Entscheidungsdilemmata sowie in Langfristperspektive (ebd.).

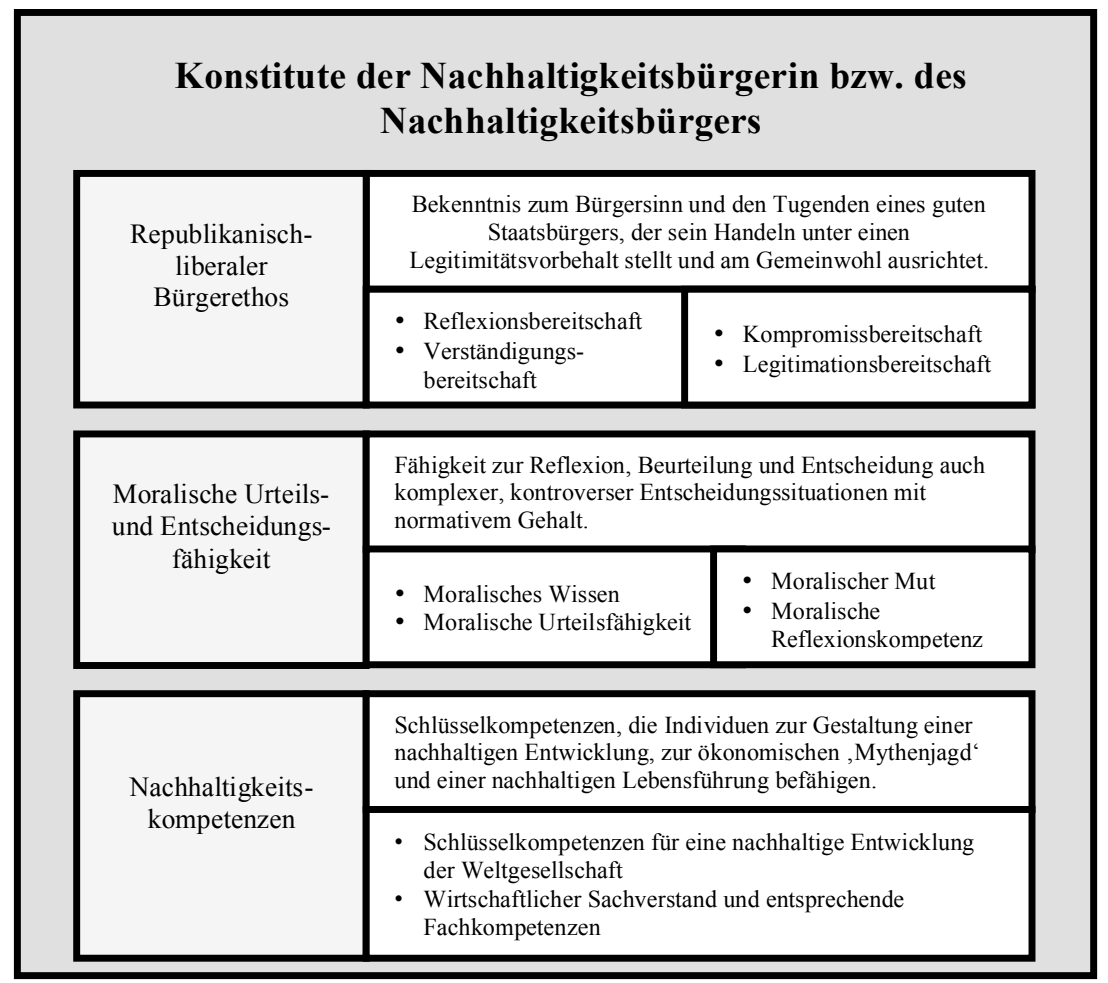

Abb. 1: Konstitute der Nachhaltigkeitsbürgerin bzw. des Nachhaltigkeitsbürgers (Rieckmann \& Schank, 2016)

Das Vorhandensein von nachhaltigkeitsrelevanten Schlüsselkompetenzen allein reicht aber nicht aus; es braucht auch guten Willen und Urteilsfähigkeit. Denn wenn es dem Individuum an der Bereitschaft mangelt, sein Handeln an Bürgersinn und Bürgertugenden auszurichten und einen Legitimitätsvorbehalt gelten zu lassen, würden die Nachhaltigkeitskompetenzen einen rein instrumentell-strategischen Charakter erhalten. Ein Handeln im Sinne einer nachhaltigen Entwicklung würde dann lediglich aus nüchtern kalkulierendem

Kapelari, Suzanne (Hg.), Vierte „Tagung der Fachdidaktik“ 2019:

„Interdisziplinäre fachdidaktische Diskurse zur Bildung für nachhaltige Entwicklung“

(C) 2020 innsbruck university press, ISBN 978-3-99106-019-2, DOI 10.15203/99106-019-2 
Eigeninteresse, unter Zwang oder aus Gründen der sozialen Erwünschtheit verfolgt. Vor diesem Hintergrund erscheint es daher sinnvoll, die Entwicklung von Nachhaltigkeitskompetenzen nicht isoliert zu betrachten und zu verfolgen, sondern sie in einem Gesamtkonstrukt einer / eines ,NachhaltigkeitsbürgerIn“ zu verorten (Rieckmann \& Schank, 2016; Wals, 2015; Wals \& Lenglet, 2016) (Abbildung 1).

Bildung für nachhaltige Entwicklung erschöpft sich also nicht in der Kompetenzentwicklung, sondern ist auch ein Bildungskonzept, das einen kritischen Wertediskurs eröffnet.

\subsection{Werteorientierung einer Bildung für nachhaltige Entwicklung}

Wenn man eine nachhaltige Entwicklung als einen gesellschaftlichen Lern-, Verständigungs- und Gestaltungsprozess begreift (Michelsen, 2007; WBGU, 2011), kann es nicht darum gehen, durch Bildungsprozesse Lernenden bestimmte Verhaltensweisen oder Werteorientierungen antrainieren zu wollen. Denn es ist Teil des Prozesses selbst, sich auf eben solche zu verständigen (Wals, 2011). Allerdings ist auch klar, dass Nachhaltigkeit keine ,theory about everything“" sein kann (Ott \& Döring, 2004) und somit auch Bildung für nachhaltige Entwicklung nicht beliebig ist.

In der Debatte über eine Wertebildung ist Bildung für nachhaltige Entwicklung in ihrer deutschsprachigen Ausprägung als ein Ansatz zu verorten, dem es nicht darum geht, vorgegebene Werte $\mathrm{zu}$ vermitteln. Gleichwohl orientiert sich Bildung für nachhaltige Entwicklung an den Ideen der intra- und intergenerationellen Gerechtigkeit (de Haan, 2008). Sie verfolgt vor diesem Hintergrund stets das wertbezogene Ziel, zu einer „Sensibilisierung für eine Überlebensverantwortung“ (Mokrosch, 2008, S. 38) beizutragen. Zugleich ist es ein pädagogisches Ziel einer Bildung für nachhaltige Entwicklung, eine Auseinandersetzung mit Werthaltungen im Sinne einer „Werteklärung“ (ebd., S. 36) $\mathrm{zu}$ ermöglichen, die mit dem Leitbild einer nachhaltigen Entwicklung verbunden sind: insbesondere in Bezug auf den Erhalt der natürlichen Lebensgrundlagen, Menschenwürde und Gerechtigkeit (Stoltenberg, 2009). Eine so verstandene Bildung für nachhaltige Entwicklung kann zu einem „Wertewandel zur Nachhaltigkeit“" (WBGU, 2011) beitragen, ohne Lernende zu

Kapelari, Suzanne (Hg.), Vierte ,Tagung der Fachdidaktik“ 2019:

„Interdisziplinäre fachdidaktische Diskurse zur Bildung für nachhaltige Entwicklung“

(C) 2020 innsbruck university press, ISBN 978-3-99106-019-2, DOI 10.15203/99106-019-2 
bevormunden oder zu überwältigen. Im Gegenteil unterstützt sie die Entwicklung einer reflexiven Kompetenz.

Von Bildung für nachhaltige Entwicklung ist also ein Beitrag zu einem kritischen Wertediskurs gleichermaßen zu erwarten und einzufordern (Rieckmann, Fischer, \& Richter, 2014; Rieckmann \& Schank, 2016). Sie kann und soll Anregungen geben, die eigenen Werte zu reflektieren und Stellung zu nehmen in der Wertedebatte auf dem Weg zu einer nachhaltigen Entwicklung (Barth, 2012). Ihr Potenzial liegt somit auch darin, den Wertehorizont der Lernenden zu erweitern. So können z.B. die lateinamerikanischen Diskurse zum Buen Vivir (Gutes Leben) und zu den Rechten der Natur in die Wertedebatte einbezogen werden (Kuhn \& Rieckmann, 2010; Rieckmann, Adomßent, Härdtle, \& Aguirre, 2011; Rieckmann, 2017). Damit können eurozentrische Denkweisen erweitert und kritisch reflektiert werden.

Für eine Transformation im Sinne einer nachhaltigen Entwicklung bedarf es aber auch struktureller und institutioneller Veränderungen (WBGU, 2011). Daher sollte Bildung für nachhaltige Entwicklung nicht nur die Ebene individuellen Verhaltens - und den dafür nötigen Wissens- und Kompetenzerwerb sowie eine entsprechende Werteorientierung - fokussieren, sondern auch die Frage nach den Strukturen, nach der „Großen Transformation“ (ebd.) aufwerfen. Bildung für nachhaltige Entwicklung sollte zur Bildung von ,NachhaltigkeitsbürgerInnen' beitragen, die die bestehenden Strukturen in Frage stellen, über diese hinausdenken und somit zur strukturellen und institutionellen Transformation beitragen (Rieckmann \& Schank, 2016; Rieckmann, 2017).

2.4 Inhalte, didaktische Prinzipien und Methoden einer Bildung für nachhaltige Entwicklung

Auch wenn im Rahmen des Konzepts einer Bildung für nachhaltige Entwicklung dem Erwerb von Kompetenzen eine zentrale Bedeutung zukommt, so sind die Themen und Inhalte, an denen sich Nachhaltigkeitskompetenzen entwickeln lassen, nicht beliebig. Themen im Rahmen einer Bildung für nachhaltige Entwicklung sollten (de Haan, 2002, S. 16f.):

- zentral für nachhaltige Entwicklungsprozesse, lokal oder global sein,

- längerfristige Bedeutung haben, 
- interdisziplinär bearbeitbar sein (d.h. es muss differenziertes Wissen aus verschiedenen Bereichen vorliegen),

- Handlungspotential aufweisen.

Wissenschaftliche Studien, gesellschaftliche Erfahrungen und die damit verbundenen Diskurse haben Handlungsfelder identifiziert, die für die Förderung einer nachhaltigen Entwicklung entscheidend sind. Diese Handlungsfelder können als wesentliche thematische Prioritäten für eine Bildung für nachhaltige Entwicklung angesehen werden. In den Zielen für nachhaltige Entwicklung (SDGs) werden 17 relevante Handlungsfelder identifiziert: Armut; Hunger; Ernährung und nachhaltige Landwirtschaft; Gesundheit und Wohlbefinden; qualitativ hochwertige Bildung; Gleichstellung der Geschlechter; sauberes Wasser und sanitäre Einrichtungen; erschwingliche und saubere Energie; menschenwürdige Arbeit und Wirtschaftswachstum; Industrie, Innovation und Infrastruktur; Verringerung der Ungleichheiten; nachhaltige Städte und Gemeinden; verantwortungsvoller Konsum und Produktion; Klimawandel; biologische Vielfalt (unter Wasser und an Land); Frieden, Gerechtigkeit und starke Institutionen; Partnerschaften für die Ziele (UNESCO, 2017). Die Roadmap zur Umsetzung des Weltaktionsprogramms ,Bildung für nachhaltige Entwicklung' listet die folgenden Schlüsselbereiche einer nachhaltigen Entwicklung auf: Klimawandel, biologische Vielfalt, Katastrophenvorsorge sowie nachhaltiger Konsum und nachhaltige Produktion (UNESCO, 2014, S. 12).

Abkommen und Programme wie die Agenda 21, das Rahmenübereinkommen der Vereinten Nationen über Klimaänderungen, das Übereinkommen über die biologische Vielfalt, der Hyogo-Aktionsplan 2005-2015 ,Building the Resilience of Nations and Communities to Disasters' und das ,Sustainable Lifestyles and Education Programme' des ,10 Year Framework of Programmes on Sustainable Consumption and Production 2012-2021' bieten weitere Informationen $\mathrm{zu}$ wichtigen Themen einer Bildung für nachhaltige Entwicklung.

Es geht also um Themen, die unseren Alltag bestimmen - Menschen jeden Alters und in unterschiedlichen Lebensbedingungen können sich mit ihnen identifizieren oder Erfahrungen in diesen Bereichen haben. Diese Themen sind komplex und vielfältig in ihren Zusammenhängen. Es ist eine Gelegenheit, sich mit ihnen in einer Art und Weise zu beschäftigen, dass das

Kapelari, Suzanne (Hg.), Vierte „Tagung der Fachdidaktik“ 2019:

„Interdisziplinäre fachdidaktische Diskurse zur Bildung für nachhaltige Entwicklung“

(C) 2020 innsbruck university press, ISBN 978-3-99106-019-2, DOI 10.15203/99106-019-2 
Transformationspotenzial im Sinne einer nachhaltigen Entwicklung zu einem zentralen Gegenstand von Bildung wird.

Bildung für nachhaltige Entwicklung geht davon aus, dass Lernprozesse selbst als Partizipationsprozesse $\mathrm{zu}$ gestalten sind und sich an Partizipation $\mathrm{zu}$ orientieren haben (Rieckmann \& Stoltenberg, 2011). Denn Kompetenzen können nicht einfach gelehrt oder unterrichtet werden; sie müssen selbst entwickelt werden (Weinert, 2001). Bildung für nachhaltige Entwicklung bedarf daher einer handlungsorientierten, transformativen Pädagogik (Rieckmann, 2018; UNESCO, 2017), die sich durch folgende didaktische Prinzipien auszeichnet (Künzli David, 2007; Littledyke \& Manolas, 2011; Rieckmann, 2018; UNESCO, 2017):

- Lernendenzentrierung und Zugänglichkeit,

- Handlungs- und Reflexionsorientierung,

- Transformatives und transgressives Lernen, ${ }^{4}$

- Partizipationsorientierung,

- Entdeckendes Lernen,

- Vernetzendes Lernen,

- Visionsorientierung,

- Verbindung von sozialem, selbstbezogenem und methodenorientiertem mit sachbezogenem Lernen.

Diese didaktischen Prinzipien stellen allgemeine Leitlinien für die Gestaltung von Lernprozessen in der Bildung für nachhaltige Entwicklung dar. Bildung für nachhaltige Entwicklung setzt eine neue Lehr- und damit auch eine Lernkultur voraus und muss auf eine große Vielfalt unterschiedlicher didaktischer Ansätze und Methoden zurückgreifen. Es bedarf Lehr- und Lernmethoden, die den genannten didaktischen Prinzipien entsprechen und somit die Entwicklung von Kompetenzen befördern. Bestimmte Methoden können für BNE besonders empfohlen werden (Rieckmann, 2018; UNESCO, 2017):

4 Transformatives Lernen zielt darauf ab, die Lernenden dazu zu befähigen, die Art und Weise zu hinterfragen und zu verändern, wie sie die Welt sehen und über sie denken (Slavich \& Zimbardo, 2012; Mezirow, 2000). Das verwandte Konzept des transgressiven Lernens (LotzSisitka, Wals, Kronlid, \& McGarry, 2015) geht noch einen Schritt weiter: Es betont, dass das Lernen in der BNE den Status quo überwinden und die Lernenden für disruptives (störendes) Denken und die Ko-Kreation neuen Wissens vorbereiten sollte.

Kapelari, Suzanne (Hg.), Vierte „Tagung der Fachdidaktik“ 2019:

„Interdisziplinäre fachdidaktische Diskurse zur Bildung für nachhaltige Entwicklung“

(C) 2020 innsbruck university press, ISBN 978-3-99106-019-2, DOI 10.15203/99106-019-2 
- Kollaborative Projekte in Ernstsituationen wie Service-Learning-Projekte und Kampagnen für verschiedene SDGs

- Methoden zur Entwicklung von Zukunftsentwürfen wie Zukunftswerkstätten, Szenarioanalysen, utopische/dystopische Erzählungen, Science-Fiction-Denken sowie Prognosen und Backcasting

- Analysen komplexer Systeme durch partizipative Forschungsprojekte, Fallstudien, Stakeholder-Analysen, Akteursanalysen, Modellierung, Systemspiele etc.

- Kritisches und reflektierendes Denken durch Fisch-Bowl-Diskussionen, Lerntagebücher etc.

Diese Lehr- und Lernmethoden ermöglichen, dass die Lernenden zu (Mit-) GestalterInnen des eigenen Lernprozesses werden und damit einen direkten Einfluss auf ihre eigene Kompetenzentwicklung haben. Zudem befähigen sie die Lernenden, Maßnahmen für eine nachhaltige Entwicklung zu ergreifen.

Um vielfältige und grenzüberschreitende Lernumgebungen zur Verfügung zu stellen, sollten Bildungseinrichtungen und Lehrende Partnerschaften auf lokaler, nationaler und internationaler Ebene fördern. Es ist wichtig zu erkennen, dass adäquate Reaktionen auf Nachhaltigkeitsprobleme nicht auf einzelne Perspektiven, Disziplinen oder Formen des Wissens beschränkt werden können. Partnerschaften, die eine Reihe von gesellschaftlichen Akteuren wie Unternehmen, NRO, öffentliche Institutionen, politische EntscheidungsträgerInnen und/oder Einzelpersonen einbeziehen, eröffnen neue Lernmöglichkeiten und werden zu einer Quelle der Kreativität und Innovation. In einem Dialog oder einem Projekt, das die Zusammenarbeit mit einem Partner in der Praxis beinhaltet, können die Lernenden etwas über realweltliche Herausforderungen erfahren und von den Kompetenzen und Erfahrungen der PartnerInnen profitieren. Partnerschaften zwischen Lernenden aus der ganzen Welt fördern den Austausch unterschiedlicher Perspektiven und Kenntnisse zu demselben Thema (UNESCO, 2017).

Diese Innovationen im Lehren und Lernen setzen allerdings bei den Lehrenden neue Lehr-Lernkompetenzen voraus (Bertschy, Künzli, \& Lehmann, 2013). Die Lehrenden müssen idealerweise selbst Nachhaltigkeitskompetenzen besitzen und diese bei ihren Lernenden entwickeln können. Das bedeutet, dass sie ein

Kapelari, Suzanne (Hg.), Vierte „Tagung der Fachdidaktik“ 2019:

„Interdisziplinäre fachdidaktische Diskurse zur Bildung für nachhaltige Entwicklung“

(C) 2020 innsbruck university press, ISBN 978-3-99106-019-2, DOI 10.15203/99106-019-2 
kritisches Verständnis von nachhaltiger Entwicklung auf der einen Seite und des pädagogischen Ansatzes von Bildung für nachhaltige Entwicklung auf der anderen Seite haben müssen. Sie benötigen Wissen über innovative LehrLernmethoden, aber auch Fähigkeiten zu deren Anwendung. Sie brauchen zudem Fähigkeiten zur Begleitung der Lernenden - z.B. in Projekten, wozu auch gehört, die eigene Rolle als Lehrende/r kritisch zu reflektieren und sich selbst eher als LernbegleiterIn zu verstehen. Im EU-Projekt, A Rounder Sense of Purpose“ - seit September 2018 in der Phase II (unter Beteiligung der Universität Vechta) - ist ein Modell für solche BNE-Kompetenzen entwickelt worden (Vare et al., 2019). ${ }^{5}$

\section{Die Rolle von Bildung für nachhaltige Entwicklung für das Erreichen der Sustainable Development Goals}

Bildung für nachhaltige Entwicklung wird in den Sustainable Development Goals (SDGs) ausdrücklich als Teil des Ziels 4.7 des SDG zu hochwertiger Bildung anerkannt - zusammen mit Global Citizenship Education (GCED), die die UNESCO als einen komplementären Ansatz fördert (UNESCO, 2015):

„Bis 2030 sicherstellen, dass alle Lernenden die notwendigen Kenntnisse und Qualifikationen zur Förderung nachhaltiger Entwicklung erwerben, unter anderem durch Bildung für nachhaltige Entwicklung und nachhaltige Lebensweisen, Menschenrechte, Geschlechtergleichstellung, eine Kultur des Friedens und der Gewaltlosigkeit, Weltbürgerschaft und die Wertschätzung kultureller Vielfalt und des Beitrags der Kultur zu nachhaltiger Entwicklung." (Vereinte Nationen, 2015, S. 19)

Gleichzeitig ist es wichtig, die zentrale Bedeutung von BNE für alle anderen 16 SDGs zu betonen. Mit ihrem Ziel, übergreifende Nachhaltigkeitskompetenzen bei den Lernenden zu entwickeln, leistet BNE einen wesentlichen Beitrag für alle Bemühungen zum Erreichen der SDGs. Denn BNE befähigt Individuen, an

5 Eine detaillierte Beschreibung dieser BNE-Kompetenzen findet sich unter: https://aroundersenseofpurpose.eu/framework/themodel/

Kapelari, Suzanne (Hg.), Vierte „Tagung der Fachdidaktik“ 2019:

„Interdisziplinäre fachdidaktische Diskurse zur Bildung für nachhaltige Entwicklung“

(C) 2020 innsbruck university press, ISBN 978-3-99106-019-2, DOI 10.15203/99106-019-2 
einer nachhaltigen Entwicklung mitzuwirken, indem sie den gesellschaftlichen, wirtschaftlichen und politischen Wandel fördern und ihr eigenes Verhalten ändern. Die Nachhaltigkeitskompetenzen machen deutlich, was ,NachhaltigkeitsbürgerInnen' insbesondere benötigen, um sich mit den heutigen komplexen Herausforderungen auseinanderzusetzen. Sie sind für alle SDGs relevant und ermöglichen es den Individuen, die verschiedenen SDGs in Beziehung zueinander zu setzen, um das große Ganze der Agenda 2030 für Nachhaltige Entwicklung zu erkennen (UNESCO, 2017).

Während die Nachhaltigkeitskompetenzen dazu befähigen, insgesamt mit den SDGs umzugehen, lässt sich für jedes einzelne SDG die Frage stellen, wie damit verbundene, spezifische Lernprozesse gestaltet werden können. BNE kann spezifische kognitive, sozioemotionale und verhaltensbezogene Lernergebnisse erzielen, die es Individuen ermöglichen, mit den besonderen Herausforderungen jedes SDG umzugehen und so dessen Umsetzung zu unterstützen (ebd.):

- Die kognitive Domäne umfasst Wissen und kognitive Fähigkeiten, die notwendig sind, um das jeweilige SDG und die mit seinem Erreichen verbundenen Herausforderungen besser zu verstehen.

- Die sozio-emotionale Domäne umfasst soziale Kompetenzen, die es Lernenden ermöglichen, zusammenzuarbeiten, $\mathrm{zu}$ verhandeln und $\mathrm{zu}$ kommunizieren, um das SDG voranzubringen, sowie Selbstreflexionsfähigkeiten, Werte, Einstellungen und Motivationen, die es den Lernenden ermöglichen, sich selbst weiterzuentwickeln.

- Die verhaltensbezogene Domäne beschreibt Handlungskompetenzen.

BNE ermöglicht es allen Individuen, zur Erreichung der SDGs beizutragen, indem sie mit den notwendigen Kenntnissen und Kompetenzen ausgestattet werden, die sie nicht nur brauchen, um zu verstehen, worum es bei den SDGs geht, sondern auch um sich als informierte BürgerInnen für die notwendige Transformation einzusetzen (ebd.). 


\section{Ganzheitlicher BNE-Ansatz}

Bildung für nachhaltige Entwicklung geht es nicht nur darum, eine nachhaltige Entwicklung in die Lehre $\mathrm{zu}$ integrieren und z.B. Schulfächern oder Studiengängen neue Inhalte hinzuzufügen. Schulen und Hochschulen sowie auch andere Bildungseinrichtungen sollten sich als Orte des Lernens und der Erfahrung für eine nachhaltige Entwicklung verstehen und daher alle ihre Prozesse an Prinzipien der Nachhaltigkeit ausrichten. Damit BNE wirksamer ist, muss die Bildungseinrichtung als Ganzes verändert werden. Ein solches ganzheitliches Konzept (Whole-Institution Approach) zielt darauf ab, Nachhaltigkeit in alle Aspekte der Bildungseinrichtung zu integrieren. Es geht darum, das Curriculum, den Betrieb, die Organisationskultur, die Beteiligung der Lernenden, die Leitung und das Management, die Beziehungen zur Kommune und die Forschung neu zu denken. Auf diese Weise fungiert die Institution selbst als Vorbild für die Lernenden. Nachhaltige Lernumgebungen wie Öko-Schulen oder grüne Campus ermöglichen es Lehrenden und Lernenden, Nachhaltigkeitsprinzipien in ihre täglichen Praktiken zu integrieren, und fördern auf umfassende Weise Kompetenzentwicklung und Wertebildung (vgl. UNESCO, 2017).

Angesichts der Bedeutung der Transformation der gesamten Bildungseinrichtung fordert das Weltaktionsprogramm (WAP) ,Bildung für nachhaltige Entwicklung' im Handlungsfeld 2 (Ganzheitliche Transformation von Lern- und Lehrumgebungen: Integration von Nachhaltigkeitsprinzipien in Bildungs- und Ausbildungskontexte) „die Förderung ganzheitlicher BNEAnsätze in Schulen und allen anderen Lern- und Lehrumgebungen“" (UNESCO, 2014, S. 18):

„BNE bedeutet viel mehr als nur eine nachhaltige Entwicklung zu predigen. Es geht darum, nachhaltige Entwicklung zu praktizieren. Nachhaltige Lernumgebungen wie Öko-Schulen oder das Prinzip ,Green Campus' geben Lehrenden und Lernenden gleichermaßen die Möglichkeit, Nachhaltigkeitsprinzipien in ihren Alltag zu integrieren. Bei der Transformation von Lern- und Lehrumgebungen geht es nicht nur darum, Einrichtungen nachhaltiger zu verwalten, sondern auch Werte und Strukturen der gesamten Institution zu verändern.“

Kapelari, Suzanne (Hg.), Vierte „Tagung der Fachdidaktik“ 2019:

„Interdisziplinäre fachdidaktische Diskurse zur Bildung für nachhaltige Entwicklung“

(C) 2020 innsbruck university press, ISBN 978-3-99106-019-2, DOI 10.15203/99106-019-2 
„Zu den Maßnahmen auf diesem Handlungsfeld gehört die Entwicklung einer Vision und eines Plans zur Umsetzung von BNE in der entsprechenden Lern- und Lehrumgebung in Zusammenarbeit mit der Gesellschaft. Leiterinnen und Leiter von Bildungsinstitutionen sind dazu aufgerufen, BNE ganzheitlich zu sehen und sich nicht nur darauf zu konzentrieren, Inhalte über nachhaltige Entwicklung zu vermitteln, sondern Nachhaltigkeit vorzuleben [...] Dabei ist die Zusammenarbeit zwischen Bildungsinstitutionen und Gemeinden sehr wichtig." (ebd.)

Ganzheitliche BNE-Ansätze sollen auf allen Ebenen und in allen Umgebungen gefördert werden. Schulen und andere Bildungseinrichtungen sowie Organisationen des öffentlichen und privaten Sektors werden ermutigt, Nachhaltigkeitspläne oder -strategien umzusetzen. Die bereits vorhandenen Erfahrungen mit ganzheitlichen BNE-Ansätzen in den Bereichen der Hochschulbildung und der Sekundarstufe sollen erweitert und auf andere Ebenen und Bildungsformen, wie z.B. frühkindliche Bildung, berufliche Bildung und non-formale Bildung für Jugendliche und Erwachsene übertragen werden. Wesentliche Elemente eines ganzheitlichen BNE-Ansatzes sind (UNESCO, 2014):

- Ein institutioneller Prozess, der es allen Stakeholdern - Leitung, Lehrenden, Lernenden, Verwaltung - ermöglicht, gemeinsam eine Vision und einen Plan zur Umsetzung von BNE in der gesamten Institution zu entwickeln.

- Technische und finanzielle Unterstützung der Institution zur Förderung ihrer Neuorientierung, einschließlich z.B. der Bereitstellung relevanter Beispiele guter Praxis, Schulungen für die Leitung und Verwaltung, die Entwicklung von Leitlinien sowie damit verbundene Forschung.

- Interinstitutionelle Netzwerke, die die gegenseitige Unterstützung wie Peerto-Peer-Lernen zu einem ganzheitlichen BNE-Ansatz fördern und die Sichtbarkeit des Ansatzes erhöhen, um ihn als Modell für die Anpassung zu verbreiten. 


\section{$5 \quad$ Nachhaltigkeitsprozess der Universität Vechta}

Die Universität Vechta ist bestrebt, das Konzept einer nachhaltigen Entwicklung in alle ihre Funktionsbereiche - Forschung, Lehre, Verwaltung, Campusgestaltung und Transfer $-\mathrm{zu}$ integrieren. Sie verfolgt somit einen Whole-Institution Approach.

Grundlage für den Nachhaltigkeitsprozess sind die Nachhaltigkeitsleitlinien der Universität Vechta, die am 21. Juni 2017 vom Senat der Universität einstimmig verabschiedet wurden. In der Präambel der Nachhaltigkeitsleitlinien heißt es:

„Die Universität Vechta verankert die Prinzipien der Nachhaltigkeit in allen ihren Bereichen - Lehre, Forschung und Campus. Aufbauend auf den Ideen der Brundtland-Kommission der Vereinten Nationen (1987) verstehen wir nachhaltige Entwicklung als eine Entwicklung, ,die den Bedürfnissen der heutigen Generation entspricht, ohne die Möglichkeiten künftiger Generationen zu gefährden, ihre eigenen Bedürfnisse zu befriedigen'. Dafür ist der Schutz der globalen Umwelt die Grundlage, was einen schonenden Umgang mit natürlichen Ressourcen sowie ein Leben und Arbeiten mit und nicht gegen die Natur voraussetzt. Neben dieser ökologischen Dimension einer nachhaltigen Entwicklung gilt es gleichermaßen, die sozialen, kulturellen, ökonomischen und politischen Perspektiven einer nachhaltigen Entwicklung $\mathrm{zu}$ berücksichtigen. Alle diese fünf Nachhaltigkeitsdimensionen müssen Beachtung finden, um innovative Nachhaltigkeitsprozesse $\mathrm{zu}$ initiieren und langfristig aufrechterhalten zu können. Die Universität Vechta schafft geeignete Rahmenbedingungen, um - im Sinne der Sustainable Development Goals (SDGs) der Vereinten Nationen - Beiträge zu einer nachhaltigen Entwicklung zu leisten. Die Nachhaltigkeitsprozesse an der Universität Vechta sind als kontinuierliche Weiterentwicklung im Sinne einer ,lernenden Organisation“ zu verstehen [...]“. (Universität Vechta, 2017)

Für diesen universitären Nachhaltigkeitsprozess werden in den Nachhaltigkeitsleitlinien fünf Handlungsbereiche benannt: Bildung für eine nachhaltige Entwicklung, Forschung für eine nachhaltige Entwicklung, Nachhaltigkeit auf 
dem Campus leben, Gemeinsames Engagement für eine nachhaltige Entwicklung, Kooperationen und Vernetzung für eine nachhaltige Entwicklung (ebd.).

In den aktuellen Zielvereinbarungen der Universität Vechta (2019-2021) mit dem Land Niedersachsen heißt es: „Die Universität verpflichtet sich, sich im Sinne der 25. Mitgliederversammlung der HRK am 06. November 2018 in Lüneburg ,Für eine Kultur der Nachhaltigkeit" einzusetzen“ (Universität Vechta, 2019, S. 5). Zudem ist Nachhaltigkeit ein wesentliches Element im aktuellen Hochschulentwicklungsplan der Universität Vechta.

Mit der AG Nachhaltige Hochschule wurde eine Arbeitsgruppe innerhalb der Universität Vechta gegründet. Diese für alle Angehörige der Universität offene Arbeitsgruppe - bestehend aus Studierenden, wissenschaftlichen MitarbeiterInnen, MitarbeiterInnen in Technik und Verwaltung und ProfessorInnen - nimmt die Aufgabe wahr, Impulse für eine nachhaltige Entwicklung an der Universität zu setzen und Empfehlungen auszusprechen.

Die Universität Vechta sieht Studium und Lehre als zentrale Mittel zur Förderung einer nachhaltigen Entwicklung an. Im Sinne des Weltaktionsprogramms ,Bildung für nachhaltige Entwicklung' sowie des neuen UNESCO-Programms ,ESD for 2030` sollen die Prinzipien einer Bildung für nachhaltige Entwicklung verstärkt in Lehre und Studium verankert werden, um Studierende auch diesbezüglich zu verantwortungsvollem Denken und Handeln zu befähigen. Den Studierenden wird die Möglichkeit gegeben, unterschiedliche Lehrveranstaltungen mit Bezug zu Nachhaltigkeitsthemen zu wählen. So gibt es z.B. im Profilierungsbereich (PB) der Universität mehrere Module zum Konzept Nachhaltige Entwicklung bzw. BNE (z.B. PB-14 Nachhaltige Entwicklung; PB15 Nachhaltige Entwicklung in der gesellschaftlichen und politischen Praxis; PBM-44 Nachhaltigkeit lernen im Geographieunterricht). Der Profilierungsbereich ist ein studiengangsübergreifendes Lehrangebot, das den Erwerb insbesondere überfachlicher Kompetenzen aus verschiedenen Bereichen und einen interdisziplinären Austausch ermöglicht. Den Studierenden wird dabei die freie Wahl gegeben, Lehrveranstaltungen nach ihren Interessen zu wählen, um so ein individuelles Kompetenzprofil zu entwickeln. Aber auch in den eigentlichen Studienfächern werden nachhaltige Entwicklung und BNE mit unterschiedlichen Schwerpunkten zum Thema gemacht. So können z.B. im Studienfach Erziehungswissenschaften im Modul ,EW-9 Professionalität durch

Kapelari, Suzanne (Hg.), Vierte „Tagung der Fachdidaktik“ 2019:

„Interdisziplinäre fachdidaktische Diskurse zur Bildung für nachhaltige Entwicklung“

(C) 2020 innsbruck university press, ISBN 978-3-99106-019-2, DOI 10.15203/99106-019-2 
erziehungswissenschaftliche Forschungs- und Methodenkompetenz Forschungsprojekte zu BNE durchgeführt werden; und im Master of Education gibt es das Modul ,BWM-6 Bildung für nachhaltige Entwicklung'. Nicht zuletzt koordiniert die Universität Vechta das ,Regional Centre of Expertise in ESD Oldenburger Münsterland “6 , und das ,Kompetenzzentrum Regionales Lernen ${ }^{7}$ der Universität Vechta beschäftigt sich ebenfalls intensiv mit BNE.

Im November 2018 hat die Universität Vechta zudem erstmals eine Aktionswoche BNE durchgeführt, die von der AG Nachhaltige Hochschule und der Vizepräsidentin für Lehre und Studium ausgerufen wurde. Alle Lehrenden der Universität waren dazu aufgerufen, während der Aktionswoche Themen und Methoden des Konzepts Bildung für nachhaltige Entwicklung in ihre reguläre Lehre zu integrieren. Dazu konnten externe ReferentInnen (mit einer Kostenübernahme durch die Universität) eingeladen werden, und es wurde eine Beratung durch BNE-erfahrene Lehrende angeboten. Zudem waren die Nutzung von bereitgestellten BNE-Materialien sowie der Austausch mit anderen Lehrenden über eine StudIP-Gruppe möglich. Die Aktionswoche BNE und eine damit verknüpfte Fortbildungsreihe zu BNE für Lehrende der Universität sollen die weitere Etablierung von BNE an der Universität fördern. Im November 2019 hat die Aktionswoche erneut stattgefunden.

Die Universität Vechta unterstützt Forschung zu nachhaltigkeitsrelevanten Fragestellungen, die der Generierung von Systemwissen (Wissen über Zusammenhänge und Mechanismen in ökologischen und sozio-ökonomischen Systemen), Zielwissen (Wissen über wünschenswerte Ziele) und Transformationswissen (Wissen zur Auslösung konkreter Veränderungsprozesse) dient. Zudem sollen sich Forschungsvorhaben in der Zielsetzung und Durchführung an Aspekten einer nachhaltigen Entwicklung orientieren. Unterschiedliche Fachbereiche an der Universität Vechta widmen sich der nachhaltigkeitsrelevanten Forschung. So ist die Universität derzeit u.a. an den Projekten ,SDGs Labs - Make the SDGs our business ${ }^{6}$, , TeRRIFICA Territorial RRI Fostering Innovative Climate Action “9 , ,A Rounder Sense of

\footnotetext{
6 http://rce-om.de/

7 https://www.uni-vechta.de/einrichtungen-von-a-z/kompetenzzentrum-regionaleslernen/startseite/

8 https://sdgs-labs.eu/

9 https://terrifica.eu/
}

Kapelari, Suzanne (Hg.), Vierte „Tagung der Fachdidaktik“ 2019:

„Interdisziplinäre fachdidaktische Diskurse zur Bildung für nachhaltige Entwicklung“

(C) 2020 innsbruck university press, ISBN 978-3-99106-019-2, DOI 10.15203/99106-019-2 
Purpose II - RSPII ${ }^{10}$ und ,Nachhaltigkeit an Hochschulen (HOCH-N): entwickeln - vernetzen - berichten ${ }^{11}$ beteiligt.

Die Universität Vechta hat zum Ziel, den Campus nachhaltig zu gestalten und Rahmenbedingungen für nachhaltiges Handeln $\mathrm{zu}$ schaffen. Es wird ein umweltschützender, ressourceneffizienter, familien- und geschlechtergerechter, inklusiver, gesundheitsförderlicher und vielfältiger Campusbetrieb angestrebt. Damit möchte die Universität eine Vorbildfunktion wahrnehmen, in der eine Nachhaltigkeitskultur und ein Bewusstsein für nachhaltiges Handeln zum Ausdruck kommen. So verwendet die Universität z.B. zu 100\% durch den Blauen Engel zertifiziertes Recyclingpapier. Die Initiative Pro Recyclingpapier, in Kooperation mit der Kompetenzstelle für nachhaltige Beschaffung des Bundesinnenministeriums sowie dem Umweltbundesamt, hat deshalb die Universität Vechta im Jahr 2015 erstmals dafür ausgezeichnet. Im Jahr werden über 2,5 Millionen Blatt Papier an der Universität Vechta verbraucht. Durch die Verwendung von Recyclingpapier anstatt von Frischfaserpapier können im Herstellungsprozess dieser Papiermenge über 395.000 Liter Wasser gespart werden. Zudem achtet die Universität Vechta bei der Beschaffung neuer ITGeräte auf energieeffiziente Kriterien, und mit der Unterstützung der Oldenburgischen Landesbank AG (OLB) konnte die Universität ein erstes $E$ Bike anschaffen, das der Auftakt für eine E-Flotte sein soll. Bereits seit 2012 beteiligt sich die Universität Vechta am Verfahren ,audit familiengerechte hochschule'. Hiermit verpflichtet sich die Universität, die Vereinbarkeit von Studium, Beruf und Familie zu gewährleisten und mit Aktivitäten und Maßnahmen zu erleichtern. Auch gibt es an der Universität studentische Initiativen, die eine nachhaltige Entwicklung befördern - z.B. die FoodSharing-Initiative ,Studentenfutter ${ }^{12}$ und ein studentischer Gemüsegarten. Derzeit ist die Universität damit befasst, einen ersten Nachhaltigkeitsbericht zu erstellen, der sich am Deutschen Nachhaltigkeitskodex für Hochschulen $(\text { Hochschul-DNK) })^{13}$ orientieren wird und im Jahr 2020 veröffentlicht werden soll.

$10 \mathrm{https}: / / \mathrm{www}$. aroundersenseofpurpose.eu/

11 https://hoch-n.org/

12 https://www.instagram.com/studentenfutter uni vechta

13 https://www.deutscher-nachhaltigkeitskodex.de/de-DE/Home/DNK/Hochschul-DNK

Kapelari, Suzanne (Hg.), Vierte „Tagung der Fachdidaktik“ 2019:

„Interdisziplinäre fachdidaktische Diskurse zur Bildung für nachhaltige Entwicklung“

(C) 2020 innsbruck university press, ISBN 978-3-99106-019-2, DOI 10.15203/99106-019-2 
Deutlich befördert wird der Nachhaltigkeitsprozess der Universität Vechta durch die Mitwirkung im Projekt, Nachhaltigkeit an Hochschulen (HOCH-N): entwickeln - vernetzen - berichten' $(2016-2020)^{14}$. Dieses Projekt widmet sich der Verankerung von Prozessen zur Entwicklung (Implementierung und Etablierung) von Nachhaltigkeit an deutschen Hochschulen. HOCH-N verbindet praktische und wissenschaftliche Ansprüche. Es bietet neben einem begleitenden Rahmenprogramm für einen gezielten, methodisch gestützten Erfahrungs- und Wissensaustausch auch ein systematisches Vernetzungsangebot unter den Hochschulen. Das Projekt verfolgt als übergeordnetes Ziel, Expertenwissen und Prozesskompetenzen zu bündeln, aufzubereiten sowie weiter auszubauen, um diese für Universitäten und Fachhochschulen nutzbar machen zu können.

Am vom Bundesministerium für Bildung und Forschung (BMBF) geförderten Projekt sind Teams aus elf deutschen Hochschulen beteiligt, die in sich in sechs Arbeitspaketen der Untersuchung und Förderung von hochschulischen Nachhaltigkeitsprozessen in den Bereichen Forschung, Lehre, Betrieb, Nachhaltigkeitsberichterstattung, Governance und Transfer widmen. Das Vechtaer Team um Prof. Dr. Marco Rieckmann bearbeitet gemeinsam mit Prof.in Dr.in Inka Bormann (Freie Universität Berlin) und ihrem Team das Arbeitspaket ,Governance', in dem die Strukturen und Prozesse untersucht werden, die der Implementierung von Nachhaltigkeit in den unterschiedlichen Handlungsfeldern einer Hochschule dienen (Bauer et al., 2018, 2020; Niedlich et al., 2019, 2020). Praxisorientierte Ergebnisse des Arbeitspakets ,Governance', aber auch der anderen Arbeitspakete sind in Leitfäden veröffentlicht worden. ${ }^{15}$

\section{Fazit}

Bildung für nachhaltige Entwicklung hat eine wichtige Bedeutung für die Förderung einer nachhaltigen Entwicklung der Gesellschaft. Sie ermöglicht Lernenden, nachhaltigkeitsrelevante Kompetenzen $\mathrm{zu}$ entwickeln und sich kritisch mit gesellschaftlichen Werten auseinanderzusetzen. Viele Bildungs-

14 https://hoch-n.org/

15 https://www.hochn.uni-hamburg.de/leitfaeden.html

Kapelari, Suzanne (Hg.), Vierte „Tagung der Fachdidaktik“ 2019:

„Interdisziplinäre fachdidaktische Diskurse zur Bildung für nachhaltige Entwicklung“

(C) 2020 innsbruck university press, ISBN 978-3-99106-019-2, DOI 10.15203/99106-019-2 
einrichtungen widmen sich bereits einer Bildung für nachhaltige Entwicklung, oft aber eher noch in Projekten. Eine Verankerung in den Strukturen steht häufig noch aus.

Der Whole-Institution Approach kann als eine Leitlinie für eine umfassende Integration von BNE dienen und muss mit einem umfassenden Nachhaltigkeitsprozess einhergehen. Das Beispiel der Universität Vechta zeigt, wie ein Whole-Institution Approach in der Praxis umgesetzt werden kann. Netzwerke wie HOCH-N haben eine wichtige Funktion für den Austausch und die Dissemination von Beispielen Guter Praxis.

Auf dem Weg zu einer tatsächlichen Transformation des Bildungssystems im Sinne des SDG 4 sind noch viele Schritte zurückzuzulegen. Das neue UNESCO-Programm ,ESD for 2030` wird dafür hoffentlich weitere Impulse setzen.

\section{Literatur}

Barth, M. (2012): Social Learning Instead of Educating the Other, GAIA 19(3), S. 9194.

Barth, M., Godemann, J., Rieckmann, M., \& Stoltenberg, U. (2007): Developing Key Competencies for Sustainable Development in Higher Education, International Journal of Sustainability in Higher Education 8(4), S. 416-430.

Bauer, M., Bormann, I., Kummer, B., Niedlich, S., \& Rieckmann, M. (2018): Sustainability Governance at Universities: Using a Governance Equalizer as a Research Heuristic, Higher Education Policy 31(4), S. 491-511.

Bauer, M., Niedlich, S., Rieckmann, M., Bormann, I., \& Jaeger, L. (2020): Interdependencies of Culture and Functions of Sustainability Governance at Higher Education Institutions, Sustainability 12(7), S. 2780.

Bertschy, F., Künzli, C., \& Lehmann, M. (2013): Teachers' Competencies for the Implementation of Educational Offers in the Field of Education for Sustainable Development, Sustainability 5(12), S. 5067-5080.

Bögeholz, S. (2007): Bewertungskompetenz für systematisches Entscheiden in komplexen Gestaltungssituationen Nachhaltiger Entwicklung. In: Krüger, D., \& Vogt, H. (Eds.): Theorien in der biologiedidaktischen Forschung, Ein Handbuch für Lehramtsstudenten und Doktoranden, Berlin, Heidelberg: Spinger, S. 209-220.

Chur, D. (2012): Kompetenzorientierung im Studium und der Erwerb von Schlüsselkompetenzen. In: Kossek, B., \& Zwiauer, C. (Eds.): Universität in Zeiten

Kapelari, Suzanne (Hg.), Vierte „Tagung der Fachdidaktik“ 2019:

„Interdisziplinäre fachdidaktische Diskurse zur Bildung für nachhaltige Entwicklung“

(C) 2020 innsbruck university press, ISBN 978-3-99106-019-2, DOI 10.15203/99106-019-2 
von Bologna, Zur Theorie und Praxis von Lehr- und Lernkulturen, Göttingen: V\& R unipress, S. 289-314.

de Haan, G. (2002): Die Kernthemen der Bildung für eine nachhaltige Entwicklung, ZEP - Zeitschrift für internationale Bildungsforschung und Entwicklungspädagogik 25(1), S. 13-20.

de Haan, G. (2008): Gestaltungskompetenz als Kompetenzkonzept der Bildung für nachhaltige Entwicklung. In: Bormann, I., \& de Haan, G. (Eds.): Kompetenzen der Bildung für nachhaltige Entwicklung: Operationalisierung, Messung, Rahmenbedingungen, Befunde, Wiesbaden: VS Verlag für Sozialwissenschaften, S. 23-43.

de Haan, G., Kamp, G., Lerch, A., Martignon, L., Müller-Christ, G., \& Nutzinger, H. G. (Eds.) (2008): Nachhaltigkeit und Gerechtigkeit, Grundlagen und schulpraktische Konsequenzen. Berlin, Heidelberg: Springer.

Dlouhá, J., Heras, R., Mulà, I., Perez Salgado, F., \& Henderson, L. (2019): Competences to Address SDGs in Higher Education - A Reflection on the Equilibrium between Systemic and Personal Approaches to Achieve Transformative Action, Sustainability 11(13), doi:10.3390/su11133664.

Erpenbeck, J., \& Heyse, V. (2007): Die Kompetenzbiographie, Wege der Kompetenzentwicklung. Münster, New York, München, Berlin: Waxmann Verlag.

Gräsel, C., Bormann, I., Schütte, K., Trempler, K., Fischbach, R., \& Asseburg, R. (2012): Perspektiven der Forschung im Bereich Bildung für nachhaltige Entwicklung. In: BMBF - Bildungsministerium für Bildung und Forschung (Eds.): Bildungsforschung Band 39: Beiträge der Bildungsforschung, Bonn, Berlin: BMBF, S. 7-24.

Heil, F. (2007): Der Kompetenzbegriff in der Pädagogik, Ein Ansatz zur Klärung eines strapazierten Begriffs. In: Heffels, W., Streffer, D., \& Häusler, B. (Eds.): Macht Bildung kompetent? Handeln aus Kompetenz - pädagogische Perspektiven. Opladen, Farmington Hills: Budrich, 43-79.

Heinrichs, H. (2007): Kultur-Evolution: Partizipation und Nachhaltigkeit. In: Michelsen, G. (Ed.): Handbuch Nachhaltigkeitskommunikation. Grundlagen und Praxis. 2. Auflage. München: Oekom-Verlag, 715-726.

Kercher, J. (2015): Wie die Nachhaltigkeitsziele in die Welt kamen. Von den MDGs zu den SDGs. In: oekom e.V. - Verein für ökologische Kommunikation (Hrsg.): Nachhaltige Entwicklungsziele. Agenda für eine bessere Welt?. München: Oekom, 27-33.

Klafki, W. (2007): Neue Studien zur Bildungstheorie und Didaktik, Zeitgemäße Allgemeinbildung und kritisch-konstruktive Didaktik, Weinheim, Basel: Beltz.

Klieme, E., \& Hartig, J. (2007): Kompetenzkonzepte in den Sozialwissenschaften und im erziehungswissenschaftlichen Diskurs. In: Prenzel, M., Gogolin, I., \& Krüger,

Kapelari, Suzanne (Hg.), Vierte „Tagung der Fachdidaktik“ 2019:

„Interdisziplinäre fachdidaktische Diskurse zur Bildung für nachhaltige Entwicklung“

(C) 2020 innsbruck university press, ISBN 978-3-99106-019-2, DOI 10.15203/99106-019-2 
H. H. (Eds.): Kompetenzdiagnostik. Zeitschrift für Erziehungswissenschaft, Sonderheft 8, Wiesbaden: Springer, 11-29.

KMK - Kultusministerkonferenz, \& BMZ - Bundesministerium für wirtschaftliche Zusammenarbeit und Entwicklung (2015): Orientierungsrahmen für den Lernbereich Globale Entwicklung im Rahmen einer Bildung für nachhaltige Entwicklung, http://www.kmk.org/fileadmin/veroeffentlichungen beschluesse/2015/2015 060 0-Orientierungsrahmen-Globale-Entwicklung.pdf, abgerufen: 5.08.2019.

Kopfmüller, J., Brandl, V., Jörissen, J., Paetau, M., Banse, G., Coenen, R., \& Grunwald, A. (2001): Nachhaltige Entwicklung integrativ betrachtet: konstitutive Elemente, Regeln, Indikatoren. Berlin: edition sigma.

Krüger, H.-H. (2012): Einführung in Theorien und Methoden der Erziehungswissenschaft, Opladen, Toronto: Budrich.

Kuhn, K., \& Rieckmann, M. (2010): Der lateinamerikanische Nachhaltigkeitsdiskurs von der Kapitalismuskritik zum „Guten Leben“. In: ITB infoservice. Berichterstattung zur Forschungs-, Technologie- und Innovationspolitik weltweit, 2. Schwerpunktausgabe 7/10, S. 8-10.

Künzli David, C. (2007): Zukunft mitgestalten. Bildung für eine nachhaltige Entwicklung - Didaktisches Konzept und Umsetzung in der Grundschule, Bern: Haupt-Verlag.

Littledyke, M., \& Manolas, E. (2011): Education for Sustainability Pedagogy: Ideological and Epistemological Barriers and Drivers. In: Leal Filho, W. (Ed.): World trends in education for sustainable development, Frankfurt am Main: Springer, S. 77-104.

Lotz-Sisitka, H., Wals, A. E., Kronlid, D., \& McGarry, D., (2015): Transformative, transgressive social learning: rethinking higher education pedagogy in times of systemic global dysfunction, Current Opinion in Environmental Sustainability 16, S. 73-80.

Lozano, R., Merrill, M., Sammalisto, K., Ceulemans, K., \& Lozano, F. (2017): Connecting Competences and Pedagogical Approaches for Sustainable Development in Higher Education. A Literature Review and Framework Proposal. Sustainability, 9 (11).

Messner, D., \& Scholz, I. (2015): Gemeinsam für das Wohlergehen aller. Agenda 2030. In: oekom e.V. - Verein für ökologische Kommunikation (Hrsg.): Nachhaltige Entwicklungsziele. Agenda für eine bessere Welt?. München: Oekom, 18-26.

Mezirow, J. (2000): Learning as transformation: critical perspectives on a theory in progress. San Francisco: Jossey-Bass.

Michelsen, G. (2007): Nachhaltigkeitskommunikation: Verständnis - Entwicklung Perspektiven. In: Michelsen, G. (Ed.): Handbuch Nachhaltigkeitskommunikation. Grundlagen und Praxis. 2. Auflage. München: Oekom-Verlag, 25-41.

Kapelari, Suzanne (Hg.), Vierte „Tagung der Fachdidaktik“ 2019:

„Interdisziplinäre fachdidaktische Diskurse zur Bildung für nachhaltige Entwicklung“

(C) 2020 innsbruck university press, ISBN 978-3-99106-019-2, DOI 10.15203/99106-019-2 
Michelsen, G. (2009): Kompetenzen und Bildung für nachhaltige Entwicklung. In: Overwien, B., \& Rathenow, H.-F. (Eds.): Globalisierung fordert politische Bildung, Politisches Lernen im globalen Kontext, Opladen, Farmington Hills: Budrich, S. 75-86.

Michelsen, G., \& Rieckmann, M. (2014): Kompetenzorientiertes Lehren und Lernen an Hochschulen, Veränderte Anforderungen und Bedingungen für Lehrende und Studierende. In: Keuper, F., \& Arnold, H. (Eds): Campus Transformation, Education, Qualification \& Digitalization, Berlin: Logos Verlag, S. 45-65.

Mogensen, F., \& Schnack, K. (2010): The action competence approach and the 'new' discourses of education for sustainable development, competence and quality criteria, Environmental Education Research 16 (1), S. 59-74.

Mokrosch, R. (2008): Zum Verständnis von Werte-Erziehung: Aktuelle Modelle für die Schule. In: Mokrosch, R., \& Regenbogen, A. (Eds.): Werte-Erziehung und Schule. Ein Handbuch für Unterrichtende, Göttingen: Vandenhoeck \& Ruprecht, S. 32-40.

Mulder, K. (2014): Strategic competencies, critically important for Sustainable Development, Journal of Cleaner Production 78, 243-248.

Niedlich, S., Kummer, B., Bauer, M., Rieckmann, M., \& Bormann, I. (2019): Cultures of sustainability governance in higher education institutions: A multi-case study of dimensions and implications, Higher Education Quarterly 00 (First published: 16 December 2019), S. 1-18.

Niedlich, S., Bauer, M., Doneliene, M., Jaeger, L., Rieckmann, M., \& Bormann, I. (2020): Assessment of Sustainability Governance in Higher Education Institutions - a Systemic Tool using a Governance Equalizer, Sustainability 12(5), S. 1816.

Ott, K., \& Döring, R. (2004): Theorie und Praxis starker Nachhaltigkeit. Marburg: Metropolis Verlag.

Overwien, B. (2013): Kompetenzmodelle im Lernbereich „Globale Entwicklung“ Bildung für nachhaltige Entwicklung. In: Overwien, B., \& Rode, H. (Eds.): Bildung für nachhaltige Entwicklung: Lebenslanges Lernen, Kompetenz und gesellschaftliche Teilhabe. Leverkusen, Opladen: Budrich, 13-34.

Rieckmann, M. (2010): Die globale Perspektive der Bildung für eine nachhaltige Entwicklung, Eine europäisch-lateinamerikanische Studie zu Schlüsselkompetenzen für Denken und Handeln in der Weltgesellschaft, Berlin: ökom.

Rieckmann, M. (2011): Schlüsselkompetenzen für eine nachhaltige Entwicklung der Weltgesellschaft, Ergebnisse einer europäisch-lateinamerikanischen DelphiStudie, GAIA 20(1), S. 48-56.

Rieckmann, M. (2013): Schlüsselkompetenzen für eine nachhaltige Entwicklung. POLIS Heft, 4, 11-14.

Rieckmann, M. (2016): Bildung für nachhaltige Entwicklung - Konzeptionelle Grundlagen und Stand der Implementierung. In: Schweer, M. (Ed.): Bildung für

Kapelari, Suzanne (Hg.), Vierte „Tagung der Fachdidaktik“ 2019:

„Interdisziplinäre fachdidaktische Diskurse zur Bildung für nachhaltige Entwicklung“

(C) 2020 innsbruck university press, ISBN 978-3-99106-019-2, DOI 10.15203/99106-019-2 
nachhaltige Entwicklung in pädagogischen Handlungsfeldern. Grundlagen, Verankerung und Methodik in ausgewählten Lehr-Lern-Kontexten. Frankfurt am Main, 11-32.

Rieckmann, M. (2017): Bildung für nachhaltige Entwicklung in der Großen Transformation - Neue Perspektiven aus den Buen Vivir- und Postwachstumsdiskursen. In: Emde, O., Jakubczyk, U., Kappes, B., \& Overwien, B. (Eds.): Mit Bildung die Welt verändern? Globales Lernen für eine nachhaltige Entwicklung. Opladen, Berlin, Toronto: Budrich, S. 147-159.

Rieckmann, M. (2018): Chapter 2 - Learning to transform the world: key competencies in ESD. In: Leicht, A., Heiss, J., \& Byun, W. J. (Eds.): Issues and trends in Education for Sustainable Development, Paris: UNESCO, http://unesdoc.unesco.org/images/0026/002614/261445E.pdf, abgerufen: 5.08.2018, S. 39-59.

Rieckmann, M., Adomßent, M., Härdtle, W., \& Aguirre, P. (2011): Sustainable Development and Conservation of Biodiversity Hotspots in Latin America, The Case of Ecuador. In: Zachos, F. E., \& Habel, J. C. (Eds.): Biodiversity Hotspots, Distribution and Protection of Conservation Priority Areas, Berlin, Heidelberg: Springer, S. 435-452.

Rieckmann, M., Fischer, D., \& Richter, S. (2014): Nachhaltige Ernährung im Wertediskurs - Beiträge einer Hochschulbildung für nachhaltige Entwicklung. In: Schank, C., Vorbohle, K., \& Quandt, J. H. (Hrsg.): Perspektive Nahrungsmittelethik. München, Mering: Rainer Hampp Verlag, S. 29-58.

Rieckmann, M., \& Schank, C. (2016): Sozioökonomisch fundierte Bildung für nachhaltige Entwicklung - Kompetenzentwicklung und Werteorientierungen zwischen individueller Verantwortung und struktureller Transformation, SOCIENCE, 1 (1), 65-79.

Rieckmann, M., \& Stoltenberg, U. (2011): Partizipation als zentrales Element von Bildung für eine nachhaltige Entwicklung. In: Kuhn, K., Newig, J., \& Heinrichs, H. (Eds.), Nachhaltige Entwicklung? Welche Rolle für Partizipation und Kooperation? Wiesbaden: VS Verlag, S. 119-131.

Rychen, D. (2003): Key competencies, Meeting important challenges in life. In: Rychen, D., \& Salganik, L. (Eds.): Key competencies for a successful life and well-functioning society, Cambridge, Toronto, Bern, Göttingen: Hogrefe und Huber, S. 63-107.

Schneidewind, U. (2013): Transformative Literacy. Gesellschaftliche Veränderungsprozesse verstehen und gestalten, GAIA 22(2), S. 82-86.

Scholz, I. (2015): Universelle Ziele für eine nachhaltige Entwicklung. In: Simonis, U. E., Leitschuh, H., Michelsen, G., Sommer, J., \& von Weizsäcker, E. U. (Eds.): Gesucht: Weltumweltpolitik. Herausforderungen im Anthropozän. Stuttgart: Hirzel, 116-125.

Kapelari, Suzanne (Hg.), Vierte „Tagung der Fachdidaktik“ 2019:

„Interdisziplinäre fachdidaktische Diskurse zur Bildung für nachhaltige Entwicklung“

(C) 2020 innsbruck university press, ISBN 978-3-99106-019-2, DOI 10.15203/99106-019-2 
Scholz, I. (2017): Herausforderung Sustainable Development Goals. In: Michelsen, G. (Ed.): Die deutsche Nachhaltigkeitsstrategie. Wegweiser für eine Politik der Nachhaltigkeit. Wiesbaden: Hessische Landeszentrale für politische Bildung, 2339.

Slavich, G. M., \& Zimbardo, P. G. (2012): Transformational Teaching: Theoretical Underpinnings. Basic Principles, and Core Methods. Educational Psychology Review 24(4), S. 569-608.

SRU - Rat von Sachverständigen für Umweltfragen (1994): Umweltgutachten 1994. Für eine dauerhaft-umweltgerechte Entwicklung. Bundestagsdrucksache 12/6995 vom 8.3.1994. Bonn: Heger,

https://www.umweltrat.de/SharedDocs/Downloads/DE/01 Umweltgutachten/1994 2000/1994 Umweltgutachten Bundestagsdrucksache.pdf;jsessionid=7EC95EB2 1779199607E98D6CE1F92B92.1 cid292? blob=publicationFile\&v=4, abgerufen 5.08.2019.

Stoltenberg, U. (2009): Mensch und Wald. Theorie und Praxis einer Bildung für nachhaltige Entwicklung am Beispiel des Themenfeldes Wald, München: ökom.

Thomas, I., Barth, M., \& Day, T. (2013): Education for Sustainability, Graduate Capabilities, Professional Employment: How They All Connect, Australian Journal of Environmental Education 29 (1), S. 33-51.

UN Environment (2019): Global Environment Outlook GEO-6: Healthy Planet, Healthy People. Cambridge: Cambridge University Press, https://www.unenvironment.org/resources/global-environment-outlook-6, abgerufen 5.08.2019.

UNESCO - United Nations Educational, Scientific and Cultural Organization (2009): Bonner Erklärung, Paris: UNESCO, https://www.unesco.de/sites/default/files/2018-03/2009 Bonner Erklaerung.pdf, abgerufen 5.08.2019.

UNESCO - United Nations Educational, Scientific and Cultural Organization (2014): UNESCO Roadmap zur Umsetzung des Weltaktionsprogramms „Bildung für nachhaltige Entwicklung“, Paris: UNESCO, https://www.bmbf.de/files/2015_Roadmap_deutsch.pdf, abgerufen 5.08.2019.

UNESCO - United Nations Educational, Scientific and Cultural Organization (2015): Global Citizenship Education: Topics and learning objectives. Paris: UNESCO, http://unesdoc.unesco.org/images/0023/002329/232993e.pdf, abgerufen 5.08.2019.

UNESCO - United Nations Educational, Scientific and Cultural Organization (2017). Education for Sustainable Development Goals. Learning Objectives. Paris: UNESCO, http://unesdoc.unesco.org/images/0024/002474/247444e.pdf, abgerufen 5.08.2019. 
Universität Vechta (2017): Nachhaltigkeitsleitlinien der Universität Vechta, https://www.uni-vechta.de/uni/nachhaltigehochschule/home/nachhaltigkeitsleitlinien, abgerufen: 5.08.2019.

Universität Vechta (2019): Zielvereinbarungen der Universität Vechta (2019 - 2021), https://www.univechta.de/fileadmin/user_upload/Praesidium/Dokumente/Zielvereinbarung_20192021.pdf, abgerufen: 5.08.2019.

Vare, P., Arro, G., de Hamer, A., Del Gobbo, G., de Vries, G., Farioli, F., ... \& Nijdam, C. (2019): Devising a Competence-Based Training Program for Educators of Sustainable Development: Lessons Learned, Sustainability 11(7), DOI: 10.3390/su11071890.

Vare, P., \& Scott, W. (2007): Learning for a Change: Exploring the Relationship between Education and Sustainable Development, Journal of Education for Sustainable Development, 1 (2), 191-198.

Vereinte Nationen (2015): Transformation unserer Welt: die Agenda 2030 für nachhaltige Entwicklung, http://www.un.org/depts/german/gv-70/band1/ar70001.pdf, abgerufen: 5.08.2019.

Wals, A. E. (2010): Mirroring, Gestaltswitching and transformative social learning, stepping stones for developing sustainability competence, International Journal of Sustainability in Higher Education 11 (4), S. 380-390.

Wals, A. E. (2011): Learning Our Way to Sustainability, Journal of Education for Sustainable Development, 5 (2), 177-186.

Wals, A. E. (2015): Beyond unreasonable doubt. Education and learning for socioecological sustainability in the Anthropocene. Wageningen, Wageningen University, https://arjenwals.files.wordpress.com/2016/02/8412100972 rvb inauguratiewals oratieboekje v02.pdf, abgerufen 5.08.2019.

Wals, A. E., \& Lenglet, F. (2016): Sustainability citizens: Collaborative and disruptive social learning. In: Horne, R., Fien, J., Beza, B. B., \& Nelson, A. (Eds.): Sustainability Citizenship in Cities: Theory and Practice, London: Routledge, 5266.

WBGU - Wissenschaftlicher Beirat der Bundesregierung Globale Umweltveränderungen (2011): Welt im Wandel: Gesellschaftsvertrag für eine große Transformation, Berlin: WBGU.

Weinert, F. E. (2001): Concept of Competence: A Conceptual Clarification. In: Rychen, D. S., \& Salganik, L. H. (Eds.): Defining and Selecting Key Competencies. Seattle, Toronto, Bern, Göttingen: Hogrefe und Huber, 45-65.

Wiek, A., Withycombe, L., \& Redman, C. (2011): Key competencies in sustainability: a reference framework for academic program development, Sustainability Science 6(2), S. 203-218.

Kapelari, Suzanne (Hg.), Vierte „Tagung der Fachdidaktik“ 2019:

„Interdisziplinäre fachdidaktische Diskurse zur Bildung für nachhaltige Entwicklung“

(C) 2020 innsbruck university press, ISBN 978-3-99106-019-2, DOI 10.15203/99106-019-2 
Wiek, A., Bernstein, M., Foley, R., Cohen, M., Forrest, N., Kuzdas, C., Kay, B., \& Withycombe Keeler, L. (2016): Operationalising competencies in higher education for sustainable development: In: Barth, M., Michelsen, G., Thomas, I., \& Rieckmann, M. (Eds.): Routledge Handbook of Higher Education for Sustainable Development, London: Routledge, S. 241-260. 


\title{
Ein Donut, der alle satt macht? Durch visuelle Datenanalyse mit GeoGebra und Gapminder nachhaltige Entwicklung greifbar machen
}

\author{
Mag. Martin Andre, Pädagogische Hochschule Tirol

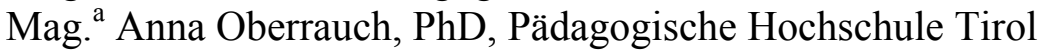 \\ Melanie Zöttl, Universität Innsbruck
}

\section{Einleitung}

Die Tagung verweist in ihrem Titel „Interdisziplinäre fachdidaktische Diskurse zu Bildung für nachhaltige Entwicklung" auf die besondere Rolle der Fachdidaktiken im Anliegen um die Gestaltung von Bildung für nachhaltige Entwicklung (BNE). Dabei sehen sich Fachdidaktiken zunehmend mit der Frage konfrontiert, wie diese Aufgabe in Schule, Hochschule und Weiterbildung nicht nur in den einzelnen Disziplinen, sondern auch interdisziplinär konzeptualisiert, erforscht und in der Praxis implementiert werden kann. (siehe Vorwort in diesem Band). Damit wird einerseits die Bedeutung der einzelnen Schulfächer, die einen Beitrag zur BNE leisten, hervorgehoben. Andererseits wird vorrangig auch der Wert von interdisziplinären Formen des Lernens betont. Aufgrund des interdisziplinären Charakters, der den Fragen rund um nachhaltige Enwicklung inhärent ist, wird dieser fächerverbindenden Perspektive große Bedeutung zu teil. Ein ähnliches Verständnis davon ist dem österreichischen Grundsatzerlass „Umweltbildung für nachhaltige Entwicklung“ (Bundesministerium für Bildung und Frauen $[\mathrm{BMBF}], 2014) \mathrm{zu}$ entnehmen, in welchem BNE ein Unterrichtsprinzip bildet, das auf die Förderung überfachlicher Kompetenzen in mehreren oder allen (Schul-)Fächern bzw. allgemein in Unterricht und Schule als Ganzes zielt. Ähnlich wurde bereits 2007 von der ständigen 
Kultusministerkonferenz der Länder in der Bundesrepublik Deutschland (KMK) und der deutschen UNESCO-Kommission (DUK) empfohlen, ,[...] im Sekundarbereich neben einer fächerverbindenden oder übergreifenden Thematisierung eine verstärkte Integration von BNE-Themen in den jeweiligen Fächern anzustreben" (KMK \& DUK, 2007, S. 4). Es wird also schon zu diesem Zeitpunkt ein gleichzeitiges Streben nach fachorientierten und fächerübergreifenden bzw. projektorientierten Implementierungsstrategien erkennbar. Für beide dieser Herangehensweisen ist es relevant, dass Fachdidaktiken ihre domänenspezifischen Zugänge und Beiträge zur BNE definieren und Unterrichtsansätze und -konzepte entwickeln, in denen die Beiträge sowohl $\mathrm{zu}$ fachspezifischem Gegenstandswissen als auch zur Förderung von Schlüsselkompetenzen für BNE (Bagoly-Simó, 2014) sichtbar werden $^{1}$. Fächer, die vermeintlich stärker mit Themen der Nachhaltigkeit verbunden sind, wie etwa der Sachunterricht in der Primarstufe, Geographie und Wirtschaftskunde, Chemie und Biologie und Umweltkunde in der Sekundarstufe, oder auch Sozialkunde, Politik und Ethik nehmen hier eine Vorreiterposition ein. Trotzdem fehlt auch im Lehrplan dieser Fächer bislang eine breite und tiefe Verankerung von Inhalten der BNE (Bagoly-Simó, 2014; Overwien, 2016).

Für andere Schulfächer, wie z.B. für die Mathematik, lässt sich bislang weder auf curricularer (Bagoly-Simó, 2014) noch auf unterrichtspraktischer Ebene feststellen, dass BNE eine relevante Leitperspektive darstellt. Dabei zeigen Untersuchungen, dass eine Integration von Themen rund um die BNE in Fächern wie etwa Mathematik und Geschichte auch ohne Verletzung curricularer Vorgaben möglich und sinnvoll ist (Niebert, 2016; Taylor et al., 2019).

1 Die AutorInnen sind sich bewusst, dass BNE nicht allein durch die Suche nach Anknüpfungspunkten in den Fächern umgesetzt werden kann, sondern die Leitidee vielmehr auch neue Perspektiven auf Inhalte und Arbeitsweisen fordert, die nur aus einer Auseinandersetzung mit dem Konzept selbst gewonnen werden können (Stoltenberg \& Fischer, 2018). Dabei werden auch nicht selten Forderungen zur Auflösung der Schulfächer und Stundentafel zugunsten von Themen gefordert, die eine inter- und transdisziplinäre Auseinandersetzung mit globalen Herausforderungen ermöglichen (Schreiner, Henriksen \& Kirkeby Hansen, 2005; Costanza et al., 2007; Hodson, 2003).

Kapelari, Suzanne (Hg.), Vierte „Tagung der Fachdidaktik“ 2019:

„Interdisziplinäre fachdidaktische Diskurse zur Bildung für nachhaltige Entwicklung“

(C) 2020 innsbruck university press, ISBN 978-3-99106-019-2, DOI 10.15203/99106-019-2 
Das im Folgenden vorgestellte Projekt One Donut for all, leistet einen Beitrag zur Einbindung der BNE in den Mathematik-Unterricht, indem eine fächerübergreifende und durch digitale Medien unterstützte Lernumgebung für den Unterricht in Mathematik und Geographie und Wirtschaftskunde (GW) entwickelt und evaluiert wird. Dabei treffen sich Mathematik- und Geographiedidaktik im Bereich der Statistik, die als jüngstes Teilgebiet der Mathematik erst in der zweiten Hälfte des 20. Jahrhunderts zusammen mit der Wahrscheinlichkeitsrechnung als das Teilgebiet ,Stochastik' Eingang in die österreichischen Lehrpläne gefunden hat. Traditionell wird im Vergleich zu anglo-amerikanischen Ländern speziell in Österreich, Deutschland oder Frankreich ein wahrscheinlichkeitstheoretischer Zugang zur Stochastik gepflegt und nur in geringem Ausmaß datenbasierte Statistik gelehrt. Die technologischen Entwicklungen seit der Jahrtausendwende hatten auch zur Folge, dass neue Konzepte für datenbasierten Statistikunterricht entwickelt und erforscht bzw. international in den Curricula verankert wurden (Garfield \& BenZvi, 2008). Streng der Tradition der Stochastik folgend, steht diese Entwicklung im deutschsprachigen Raum größtenteils noch aus.

Im Gegensatz dazu setzt sich das Fach GW im Kern mit globalen Herausforderungen nachhaltiger Entwicklung auseinander. Der Rückgriff auf umfangreiche räumliche oder raumzeitliche Daten zu ökologischen, sozialen, wirtschaftlichen Verhältnissen in der Welt ermöglicht durch Analyse, Interpretation oder (Geo-)Visualisierung der Daten statistische Perspektiven auf die Thematik, was auch für die BNE einen Mehrwert bildet.

Im vorliegenden Beitrag werden aus den Didaktiken der Mathematik sowie der Geographie und Wirtschaftskunde theoretische Grundlagen zur Entwicklung und Evaluation der fächerübergreifenden Lernumgebung vorgestellt. Nach der Einführung alternativer relevanter Nachhaltigkeitskonzeptionen wird das Modell der Donut-Ökonomie erklärt, das für das vorgestellte Unterrichtskonzept maßgebend ist. Mithilfe dieses Modells werden SchülerInnen angeleitet, statistische Fragen zu formulieren, entsprechende Daten zu sammeln und über deren Visualisierung und Interpretation die Daten auf unterschiedlichen Maßstabs- bzw. Handlungsebenen zu erkunden. Damit sollen einerseits das statistische Denken und andererseits das Denken im Sinne der nachhaltigen Entwicklung gefördert werden. Die Lernumgebung wird in ihren wesentlichen

Kapelari, Suzanne (Hg.), Vierte „Tagung der Fachdidaktik“ 2019:

„Interdisziplinäre fachdidaktische Diskurse zur Bildung für nachhaltige Entwicklung“

(C) 2020 innsbruck university press, ISBN 978-3-99106-019-2, DOI 10.15203/99106-019-2 
Phasen dargestellt und erste Eindrücke aus der Erprobung in der Sekundarstufe I werden skizziert. Der Schluss gibt einen Ausblick auf die weiteren geplanten Forschungsarbeiten.

\section{Fachdidaktische Hintergründe zum Projekt}

Neben der Darstellung der fachdidaktischen Hintergründe der Fächer Mathematik und GW und mit letzterem eng verbunden die interdisziplinären Schlüsselkompetenzen der BNE, die in den Abschnitten 2.1. und 2.2. ausgeführt werden, werden mit diesem Unterrichtsentwurf auch gezielt andere überfachliche Kompetenzen angesprochen. Besondere Erwähnung sollen hier vorab Konzepte der 21st century skills und Zugänge zur ,Digitalen Grundbildung' finden, während Erläuterungen zur BNE in Kap. 2.2. integriert werden.

In einer umfassenden Analyse verschiedener theoretischer Netzwerke zu den 21st century skills, wie etwa jenes der Partnership for 21st Century Skills (P21) oder auch jenes der Organisation für wirtschaftliche Zusammenarbeit und Entwicklung (OECD), hält Dede (2010) fest, dass alle untersuchten Konzepte größtenteils ähnliche Kompetenzen ansprechen. Diese umfassen Kompetenzen, die auf 21st century content fokussiert sind, wie etwa globales Bewusstsein oder zivil-politische Bildung und Kompetenzen, die auf Lern- und Denkprozesse abzielen, bspw. kritisches Denken und Problemlösekompetenz oder auch die Kompetenz des kreativen und innovativen Denkens. Auch Ethik, soziale Verantwortung und Selbstverantwortung werden vom Autor in den verschiedenen theoretischen Netzwerken identifiziert. Eindeutig erkennbar ist die Überschneidung der Konzepte auch im Bereich der Informations- und Kommunikationstechnologien, die von den federführenden Organisationen wie der Organisation für wirtschaftliche Zusammenarbeit und Entwicklung (OECD) als wesentlich hervorgehoben werden.

In Österreich ist Digitale Grundbildung (BGBl. II Nr. 71/2018) seit dem Schuljahr 2018/19 flächendeckend im Lehrplan der Sekundarstufe I verankert. SchülerInnen erwerben dabei in einem Ausmaß von zwei bis vier Wochenstunden innerhalb von vier Jahren meist aufgeteilt auf verschiedene

Kapelari, Suzanne (Hg.), Vierte „Tagung der Fachdidaktik“ 2019:

„Interdisziplinäre fachdidaktische Diskurse zur Bildung für nachhaltige Entwicklung“

(C) 2020 innsbruck university press, ISBN 978-3-99106-019-2, DOI 10.15203/99106-019-2 
Schulfächer Kompetenzen u.a. aus den Bereichen Mediengestaltung oder Informations-, Daten- und Medienkompetenz. Fächerverbindende Zugänge erscheinen in diesem Zusammenhang besonders geeignet, um zur Entwicklung überfachlicher Kompetenzen beizutragen. Auch der in diesem Beitrag dargestellte Unterrichtsentwurf leistet auf verschiedenen Ebenen neben der Kompetenzentwicklung in den Fächern Mathematik und GW einen Beitrag zur Entwicklung dieser überfachlichen Kompetenzen.

\subsection{Fachdidaktische Hintergründe - Mathematik und Statistik}

Im österreichischen Lehrplan der Sekundarstufe I ist Statistik in der 8. Schulstufe über das „Untersuchen und Darstellen von Datenmengen unter Verwendung statistischer Kennzahlen (z.B. Mittelwert, Median, Quartil, relative Häufigkeit, Streudiagramm)“ (BGBl. II Nr. 133/2000, Anl. A, S. 1055) verankert. Im Gegensatz zu anderen Curricula wie etwa jenem für ,Mathematik und Statistik' aus Neuseeland (Ministry of Education, 2007) oder den anerkannten Leitfäden für viele US-amerikanische Curricula (Franklin et al., 2007) sind in den österreichischen Lehrplänen und Leitfäden keine expliziten Hinweise auf prozess- und handlungsorientierte Arbeitsweisen im Bereich der Statistik zu finden, wie sie etwa aus Untersuchungen zur statistischen Arbeit (Wild \& Pfannkuch, 1999) ableitbar sind. Der dort beschriebene statistische Forschungskreislauf bietet mit den Stufen - 1) Identifizierung einer statistischen Fragestellung, 2) Datensammlung, 3) Datenanalyse und 4) Interpretation der Ergebnisse - auch im Schulunterricht der Sekundarstufe I die Möglichkeit, SchülerInnen in statistische Arbeitsweisen einzuführen, was auch prinzipiell unter dem österreichischen Lehrplan in Mathematik möglich wäre.

Einigkeit herrscht in der internationalen Forschung über die Tatsache, dass statistische Lernprozesse und die Entwicklung von Kompetenzen durch das Arbeiten in realen Kontexten befördert werden (Makar \& Ben-Zvi, 2011). Dabei wandern die Lernenden in einem durchgehenden Interpretationsprozess gedanklich zwischen ihrer Lebenswelt und der Welt der Daten hin und her und entwickeln somit tiefere Einsichten in die Beschaffenheit beider Welten, wobei diese Denkprozesse zumeist ihren Ausganspunkt im realen Kontext haben (BenZvi \& Aridor-Berger, 2016). Weitreichende Forschungsarbeiten zur

Kapelari, Suzanne (Hg.), Vierte „Tagung der Fachdidaktik“ 2019:

„Interdisziplinäre fachdidaktische Diskurse zur Bildung für nachhaltige Entwicklung“

(C) 2020 innsbruck university press, ISBN 978-3-99106-019-2, DOI 10.15203/99106-019-2 
Beschaffenheit und zu den Eigenschaften von realen Kontexten, die wesentlich dafür sind, dass SchülerInnen eine Verbindung zwischen den beiden Welten, den Daten und ihre erlebte Wirklichkeit, herstellen können, stehen aber noch aus und sind Untersuchungsgegenstände dieses Unterrichtsprojekts.

Ein weiterer aktueller Untersuchungsgegenstand in der internationalen Forschung zum Statistikunterricht ist die Bildung mit und über neue Technologien, was im direkten Zusammenhang mit der Forderung der Verwendung realer Kontexte und Daten steht. Zahlreiche Forschungsarbeiten befassen sich unter verschiedensten Blickwinkeln mit dem sinnvollen Technologieeinsatz im Statistikunterricht (Biehler et al., 2012; Wassong et al., 2014). Eindeutig daraus abzuleiten ist die Aussage, dass moderner Statistikunterricht ohne den gezielten Einsatz von Technologie nicht möglich ist. Es zeigt sich aber auch, dass der sinnvolle Einsatz von Technologie stark an die unterrichtenden LehrerInnen gebunden ist, sodass sich vorrangig auch die LehrerInnenausbildung mit diesem Thema befassen muss (Pfannkuch, 2018; Pratt et al., 2011).

Neue Technologien eröffnen neue Zugänge zur statistischen Bildung. Gal (2002) betont in seinem theoretischen Konzept der statistical literacy die Wichtigkeit der Teilhabe an zivilgesellschaftlichen und politischen Prozessen, die oft durch mediale Darstellungen von Informationen, Statistiken und Daten, die in einer großen Zahl frei zugänglich sind, kommuniziert werden. Aktuell können vermehrt Unterrichtsentwürfe gefunden werden, durch die das Thema Zivilstatistik aus den verschiedenen Blickwinkeln für den Stochastikunterricht an Schulen aufgearbeitet werden (Biehler et al., 2019), sodass diese zur Kompetenzentwicklung für mündiges Bürgertum (Gal, 2002) beitragen können. Hinsichtlich der zivilgesellschaftlichen und politischen Teilhabe sind auch Konzepte wie das statistical storytelling (Skranefjell \& Tønnessen, 2003) oder die Erstellung von Infographiken (Taspolat et al., 2017) eine Chance, den fachlichen und überfachlichen Kompetenzerwerb durch die Suche, Analyse, Interpretation und verbale oder graphische Darstellung von Informationen und Daten im Statistikunterricht zu fördern. Speziell den Infografiken wird ein großes Potenzial hinsichtlich ihres Einsatzes für den Unterricht zugesprochen (Kennedy et al., 2015).

Kapelari, Suzanne (Hg.), Vierte „Tagung der Fachdidaktik“ 2019:

„Interdisziplinäre fachdidaktische Diskurse zur Bildung für nachhaltige Entwicklung“

(C) 2020 innsbruck university press, ISBN 978-3-99106-019-2, DOI 10.15203/99106-019-2 
Fischbein (1987) untersuchte die Qualität von Intuitionen und unterstreicht deren Wert für Naturwissenschaften und Mathematik - insbesondere im Bereich der Stochastik. Diese Theorien haben schon damals zusammen mit Untersuchungen $\mathrm{zu}$ Heuristiken und systematischen Fehlern (Tversky \& Kahneman, 1974) bei Prozessen des (statistischen) Urteilens und Schlussfolgerns Eingang in Forschungsarbeiten zum Stochastikunterricht gefunden. Visualisierungen und vielfältige weitere Möglichkeit zur Arbeit mit Daten können über den Einsatz von neuen Technologien dazu beitragen, diese intuitiven Zugänge zur Statistik zu fördern (Andre et al., 2019). Auch dahingehend versucht das hier dargestellte Projekt einen Beitrag zur Forschung zu leisten, indem die intuitiven Zugänge der SchülerInnen zur Statistik unter der Verwendung neuer Technologien beachtet und analysiert werden.

Prodromou (2014) entwickelte den visuell-explorativen Forschungskreislauf (vgl. Abb. 1), der die Entwicklung von intuitiven Zugängen zu statistischen Ideen (Garfield \& Ben-Zvi, 2008), wie etwa die Idee von Zentralwerten, Streuung oder Korrelation, ermöglicht (Andre et al., 2019) und die SchülerInnen somit statistische Prozesse auf einer einfachen, visuellen Ebene selbständig verfolgen können. Die Schritte dieses Kreislaufs beginnen - wie auch in dem von Wild und Pfannkuch (1999) beschriebenen, statistischen Arbeitsablauf - mit einer statistischen Fragestellung, gehen über die Sammlung von vorhandenen Daten und das Erstellen und Interpretieren passender Grafiken bis zur Bewertung der Ergebnisse. Die Abfolge wird durch im Prozess gewonnene Einsichten oftmals auch abgeändert und wiederholt. 


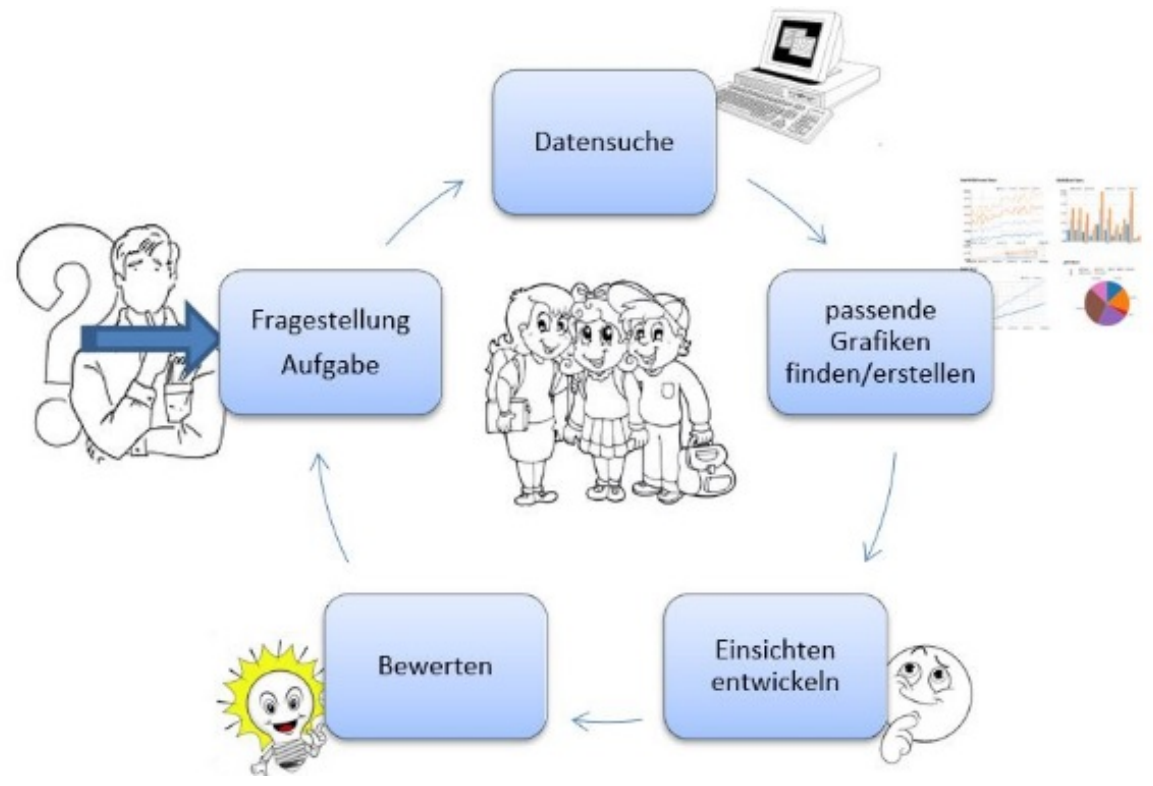

Abb. 1: Kreislauf der visuell-explorativen Datenanalyse (Eigene Darstellung A.M. nach Prodromou, 2014, Andre et al., 2019, S. 142)

Für das hier beschriebene Unterrichtsprojekt sind dieser visuell-explorative Forschungskreislauf, die Software Gapminder (https:/www.gapminder.org/ tools/) und die daran angeschlossene Bilderdatenbank Dollarstreet (https://www.gapminder.org/dollar-street/) essentiell. Die SchülerInnen beantworten statistische Forschungsfragen im Kontext der BNE, indem sie den Forschungskreislauf der visuell-explorativen Datenanalyse mit den von Gapminder frei zur Verfügung gestellten politischen, sozialen und wirtschaftlichen Daten verfolgen. Dabei nutzen die SchülerInnen ihre intuitiven Zugänge zu statistischen Ideen, um mittels nachfolgender Reflexion und Formalisierung der Lernprozesse Kompetenzen hinsichtlich des „Untersuchen[s] und Darstellen[s] von Datenmengen unter Verwendung statistischer Kennzahlen“ (BGBl. II Nr. 133/2000, Anl. A, S. 1055) zu erwerben. 
2.2 Fachdidaktische Hintergründe - Geographie und Wirtschaftskunde

Sich mit Fragen und entsprechend auch Modellen und Definitionen nachhaltiger Entwicklung auseinanderzusetzen, bildet einen wichtigen Inhalt im Fach Geographie, das oft auch als Kernfach der Nachhaltigkeitsbildung bezeichnet wird (Haubrich, Reinfried \& Schleicher, 2008; Reuschenbach \& Schockemöhle, 2011): SchülerInnen gewinnen Einsichten in komplexe Zusammenhänge von natürlichen Gegebenheiten und menschlichen Aktivitäten und entwickeln darauf aufbauend eine raumbezogene wertorientierte Handlungskompetenz. BNE bildet auch die zentrale Leitperspektive eines zukunftsorientierten GWUnterrichts, wie es im österreichischen Lehrplan der AHS-Oberstufe verdeutlicht wird:

Das Ziel der Bildung für Nachhaltige Entwicklung 'allen Menschen Bildungschancen (zu) eröffnen, die es ihnen ermöglichen, sich Wissen und Werte anzueignen sowie Verhaltensweisen und Lebensstile zu erlernen, die für eine lebenswerte Zukunft und eine positive Veränderung der Gesellschaft erforderlich sind' (UNESCO 2005, 6) sollte für gelingenden GW-Unterricht vorrangig sein. (BGB1. Nr. 219/2016, Teil II, S.62)

Gerade der Fächerkombination von Geographie und Wirtschaftskunde wohnt das Potenzial inne, Nachhaltigkeits-Themen in ihrer komplexen, systemischen Beschaffenheit bezüglich Interaktionen von Wirtschaft, Gesellschaft und Umwelt und daraus resultierender Kontroversität $\mathrm{zu}$ bearbeiten (Haubrich, Reinfried \& Schleicher, 2008; Ohl, 2013; Hemmer, 2016; Bedehäsing \& Padberg, 2017). Als Ziel der BNE wird es gesehen, Kompetenzen zu fördern, die ein bewusstes Handeln im Sinne einer nachhaltigen Entwicklung unterstützen. Individuen sollen dazu befähigt werden, an nachhaltiger Entwicklung zu partizipieren und eigene Handlungen diesbezüglich kritisch zu reflektieren (Barth, Godeman, Rieckmann \& Stoltenberg, 2007; de Haan, 2010; Rieckmann, 2011). Aus der Vielzahl vorhandener Kompetenzkonzepte für die BNE (Rychen \& Salganik, 2003; de Haan, 2010; Wiek, Withycombe \& Redman, 2011) leitet Rieckmann (2018) acht Schlüsselkompetenzen ab: 1) die Kompetenz, systemisch zu denken, 2) die Kompetenz, vorausschauend $\mathrm{zu}$ denken, 3) die normative Kompetenz, 4) die strategische Kompetenz, 5) die

Kapelari, Suzanne (Hg.), Vierte „Tagung der Fachdidaktik“ 2019:

„Interdisziplinäre fachdidaktische Diskurse zur Bildung für nachhaltige Entwicklung“

(C) 2020 innsbruck university press, ISBN 978-3-99106-019-2, DOI 10.15203/99106-019-2 
Kompetenz zu kollaborieren, 6) die Kompetenz, kritisch zu denken, 7) Selbstkompetenz und 8) die ganzheitliche Problemlösungskompetenz wurden dabei als wesentlich identifiziert.

Ein Vergleich dieses Modells mit aktuellen Kompetenzmodellen des GWUnterrichts in Österreich (BMBF, 2012) und des Geographieunterrichts für den mittleren Schulabschluss in Deutschland (Deutsche Gesellschaft für Geographie [DGFG], 2012) machen die vielfältigen Überschneidungen zum Konzept der BNE deutlich. So spielen im GW- bzw. Geographie-Unterricht Kompetenzbereiche der Multiperspektivität, Synthese/Bewertung/Beurteilung, Kommunikation, Handlung, Reflexion im Kontext nachhaltiger Entwicklung eine zentrale Rolle. Ebenso zeigen die fachdidaktischen Prinzipen der Lebenswelt- und Handlungsorientierung, Aktualitäts- und Zukunftsorientierung, Problemorientierung, Orientierung an inhaltlicher Mehrperspektivität sowie die Integration kritischer Zugänge (Fridrich \& Hofmann-Schneller, 2017) deutliche Überschneidungen in den Herangehensweisen an die BNE und an den GWUnterricht.

Noch konkreter kann eine fachdidaktische Begründung für die entwickelte Lernumgebung durch die für den GW-Unterricht relevanten Basiskonzepte (Jekel \& Pichler, 2017) gegeben werden. Als besonders relevant erachten wir dabei folgende Basiskonzepte.

- Lebensqualität und Nachhaltigkeit: Dieses Basiskonzept beinhaltet die Fähigkeit, Fragen nachhaltiger Entwicklung mit Fragen alltäglicher Lebensgestaltung und eigenen Vorstellungen guten Lebens zu verknüpfen. Damit bildet das Konzept eine tragende Säule des vorgestellten Unterrichtsentwurfs, da Lernende ihre Vorstellungen zur Auswahl von Faktoren für Lebensqualität in die Modellbildung nachhaltiger Entwicklung einbringen. Fachdidaktische Forschungsarbeiten zeigen, dass einerseits zwischen den Faktoren der Lebensqualität, die Jugendliche als relevant erachten, und andererseits den sozialen Mindeststandards, die im Modell der Donut-Ökonomie formuliert werden, zahlreiche Übereinstimmungen bestehen (Oberrauch, Keller, Sanin \& Riede, 2014). Der vorgestellte Unterrichtsentwurf orientiert sich vordergründig stark am Modell der DonutÖkonomie (Raworth, 2012), durch welches die Ebene der sozioökonomischen Grundbedürfnisse mit Fragen der ökologischen

Kapelari, Suzanne (Hg.), Vierte „Tagung der Fachdidaktik“ 2019:

„Interdisziplinäre fachdidaktische Diskurse zur Bildung für nachhaltige Entwicklung“

(C) 2020 innsbruck university press, ISBN 978-3-99106-019-2, DOI 10.15203/99106-019-2 
Belastungsgrenzen verbunden wird. Im Hintergrund kann Reflexion über Nachhaltigkeit im Sinne inter- und intragenerationeller Gerechtigkeit zum Aufbau von Vorstellungen allgemeiner und persönlicher Lebensqualität beitragen und damit wirkmächtig für BNE werden.

- Mensch-Umwelt-Beziehungen: Auch Fragen zu Mensch-UmweltBeziehungen sind im Modell der Donut-Ökonomie inhärent. Über die Bearbeitung der Frage, wie das fragile Mensch-Umwelt-System in Balance gebracht werden kann, erkennen die Lernenden das Erdsystem als ein komplexes System, das durch die Entwicklung verschiedenster Einflussfaktoren und Interaktionen zwischen diesen geprägt ist. Bei der eigenständigen Anwendung des Modells der Donut-Ökonomie können SchülerInnen ihre Fähigkeit, systemisch zu denken, weiterentwickeln, indem sie relevante Systemelemente und Wechselwirkungen zwischen diesen identifizieren und auf Basis eigener Modellierungen Prognosen und Maßnahmen zur Systemnutzung (Brockmüller et al., 2016) bzw. Handlungsoptionen ableiten (Mehren et al., 2018). Ebenso reflektieren die Lernenden über Systemgrenzen und die Relevanz von Kipppunkten und können mögliche Folgen bei deren Überschreitung bzw. die erfahrene Widerstandsfähigkeit des Erdsystems diskutieren.

- Maßstäblichkeit: Die SchülerInnen analysieren und interpretieren Daten und entwickeln Modelle vorwiegend auf ,Meso-Ebene“, also auf regionaler oder nationaler Maßstabsebene, abstrahieren und beziehen ihre Schlussfolgerungen aber auch auf weitere Handlungsebenen im Prozess nachhaltiger Entwicklung (KMK \& Bundesministerium für wirtschaftliche Zusammenarbeit und Entwicklung [BMZ], 2016). So leiten sie etwa begründete Handlungsoptionen auch auf der ,Makro-Ebene', also für die Welt als Ganzes oder ihre Kontinente sowie auf der ,Mikro-Ebene“ ihrer Lebenswelt, also für Gemeinden, Familien oder für das Individuum ab.

- Wahrnehmung und Darstellung und Interesse, Konflikte und Macht: Im GWUnterricht werden Bilder und Vorstellungen über die Welt entwickelt, die auf subjektiver bzw. intraindividueller Wahrnehmung und interessensgeleiteter Darstellung beruhen und dahingehend reflektiert werden. Dementsprechend soll das in der Lernumgebung zentrale Modell der Donut-Ökonomie nicht nur als vorgegebene Denk- und Interpretationshilfe hingenommen werden, sondern auch als verallgemeinerndes Modell, das die Wirklichkeit nicht als Gesamtes 
abbilden kann, erkannt und kritisch reflektiert werden. Indem SchülerInnen in der Anwendung des Donut-Modells etwa bei der Auswahl von Variablen und Faktoren oder bei der Definition von Schwellenwerten bewusst eigene Entscheidungen einbringen, wird die subjektive Konstruktion offenbar und gibt damit Anlass zur kritischen Reflexion des Modells und seiner Anwendung. In diesem Zusammenhang ergeben sich auch Potentiale für einen kritisch-emanzipatorischen Geographieunterricht, wie es im Konzept der Spatial-Citizenship-Education gefordert wird (Gryl \& Jekel, 2012; Jekel, Gryl \& Oberrauch, 2015). Die Verknüpfung zwischen geomedialer Visualisierung und kritischer Reflexion wird damit um die interessensgeleitete Kommunikation und Partizipation mittels digitaler Geomedien erweitert.

Neben den hier dargestellten Basiskonzepten könnten noch weitere wie Disparität und Diversität, Wachstum und Krise oder Kontingenz als fachdidaktische Begründung für den Unterrichtsentwurf herangezogen werden, auf deren Ausführung hier aber verzichtet wird.

\section{Nachhaltigkeits-Konzeptionen als Denk- und Analyse- instrumente im Unterricht}

Um statistisch-visuelle Datenanalysen zum Thema nachhaltige Entwicklung durchführen $\mathrm{zu}$ können, muss ein entsprechend geeignetes Modell zur Darstellung dieser bestimmt werden, welches die Analyse der räumlichen Daten steuert und eine altersadäquate Denk- und Interpretationshilfe zur problem- und lösungsorientierten Erarbeitung bietet.

Pufé (2017) betont, dass gerade beim komplexen Thema nachhaltiger Entwicklung verschiedene Aspekte und Themen in einen Gesamtzusammenhang gebracht werden müssen und Modelle dabei helfen können, diese Komplexität vereinfacht und verständlich darzustellen. Konkretisierungen der Idee nachhaltiger Entwicklung erfolgen dabei häufig in Form von wissenschaftlichen Nachhaltigkeitskonzeptionen (Grünwald \& Kopfmüller, 2012). Auch wenn diese Konzeptionen in den Nachhaltigkeitswissenschaften

Kapelari, Suzanne (Hg.), Vierte „Tagung der Fachdidaktik“ 2019:

„Interdisziplinäre fachdidaktische Diskurse zur Bildung für nachhaltige Entwicklung“

(C) 2020 innsbruck university press, ISBN 978-3-99106-019-2, DOI 10.15203/99106-019-2 
kontrovers diskutiert werden, haben sich mehrere Aspekte als Kern, Bezugspunkt und Grundprinzip jeglicher Nachhaltigkeitsdiskussion erwiesen. So bildet etwa der Gerechtigkeitsbegriff eine normative Grundlage oder die Dreidimensionalität von Ökonomie, Ökologie und Soziales (Pufé, 2017), ggf. ergänzt um die vierte Dimension der Politik und/oder Kultur (Grünwald \& Kopfmüller, 2012) eine grundlegende Perspektive. Uneinigkeit herrscht aber darüber, wie die Interaktion zwischen den Dimensionen darzustellen ist und wie die Gewichtung der unterschiedlichen Dimensionen zu erfolgen hat (Grünwald \& Kopfmüller, 2012). „Verschiedene Konzeptionen nachhaltiger Entwicklung sind nicht mehr oder weniger ,richtig', sondern mehr oder wenig nützlich für Reflektions- und Lernprozesse (sic!).“ (Emmrich \& Melzer, 2006, S. 173)

Im Folgenden werden jene Konzeptionen in ihren Grundzügen und einfachen, modellhaften Darstellungen beschrieben, die den wissenschaftlichen Diskurs der Nachhaltigkeit und BNE stark präg(t)en. Gegenüber der heute oftmals noch dominanten „Drei-Säulen-Metaphorik“ (Kap. 3.1.) erweist sich für das Anliegen der fächerübergreifenden Unterrichtskonzeption das Modell der DonutÖkonomie (Kap. 3.2.) als geeignet. Es vermittelt eine im aktuellen wissenschaftlichen Nachhaltigkeitsdiskurs adäquate Zugangsweise zu nachhaltiger Entwicklung, unterstützt die Erfassung, Analyse, Visualisierung und Interpretation von raumbezogenen Daten auf unterschiedlichen Maßstabsebenen und kann somit als Werkzeug zur Erkenntnisgewinnung im Unterricht genutzt werden.

\subsection{Modelle der Nachhaltigkeit}

In der historischen Betrachtung dominierten zunächst die prominent vertretenen Ein-Säulen-Modelle, die zumeist ökologischen Belangen Vorrang einräumten (Grünwald \& Kopfmüller, 2012). Die zunehmende Kritik am einseitigen Fokus dieser Modelle führte dazu, dass sich die Vorstellung einer weitestgehend gleichrangigen Bedeutung der Dimensionen Ökonomie, Ökologie und Soziales verbreitete. Im klassischen Drei-Säulen-Modell der Nachhaltigkeit (Abb. 2, links), das vielfach als Grundlage für methodisch geleitete Problembearbeitungen im Unterricht herangezogen wurde (Bundeländerkonferenz [BLK], 2003), stehen die drei Dimensionen Ökonomie, Ökologie und Soziales gleichberechtigt nebeneinander (Pufé, 2017). Nach diesem Modell ist

Kapelari, Suzanne (Hg.), Vierte ,Tagung der Fachdidaktik“ 2019:

„Interdisziplinäre fachdidaktische Diskurse zur Bildung für nachhaltige Entwicklung“

(C) 2020 innsbruck university press, ISBN 978-3-99106-019-2, DOI 10.15203/99106-019-2 
Entwicklung nachhaltig, wenn die ökologische, soziale und ökonomische Perspektive berücksichtigt wird. Dem Modell wurde jedoch eine Orientierungsfunktion abgesprochen, weil es der Vorstellung Vorschub leiste, „dass sich ökologische, ökonomische und soziale Nachhaltigkeit unabhängig voneinander realisieren ließen" (Grünwald \& Kopfmüller, 2012, S. 59). So wird auch für Bildungsprozesse die Gefahr gesehen, innerhalb der Säulenlogik verhaftet zu bleiben und mehrperspektivische Betrachtungsweisen unzureichend abzubilden (Emmrich \& Melzer, 2006). Demgegenüber versucht das DreiklangModell (Abb. 2, Mitte) den unauflösbaren Zusammenhang unter den Nachhaltigkeitsdimensionen sichtbar zu machen. Die Überlappungen zwischen ökonomischer, sozialer und ökologischer Dimension veranschaulichen die fließenden Grenzen zwischen den Dimensionen. Die Überlappungsbereiche benennen jeweils die Kernwerte der Überschneidungen (Pufé, 2017).
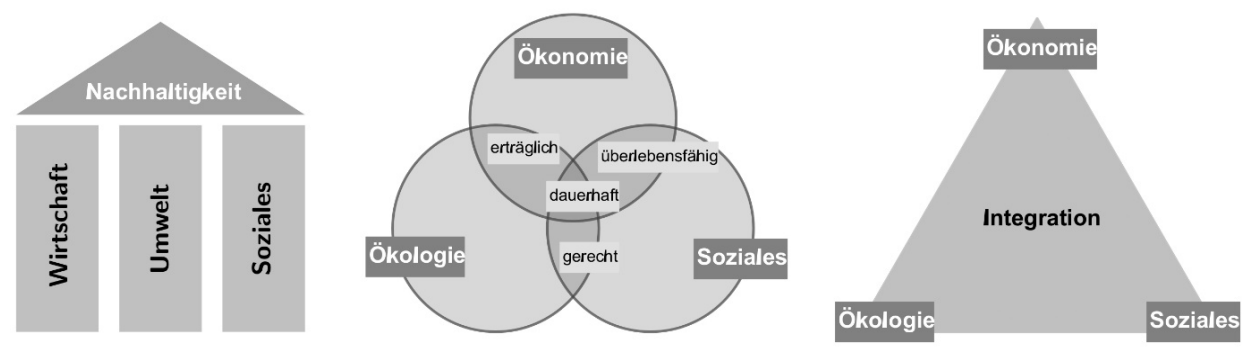

Abb. 2: Das Drei-Säulen-Modell, Dreiklang-Modell und integratives Nachhaltigkeitsdreieck im direkten Vergleich (Eigene Darstellung A. M. nach Pufé, 2017, S. 118, S.121-122)

Aus diesen Modellen ist schließlich das Modell des integrativen Nachhaltigkeitsdreiecks hervorgegangen (Abb. 2, rechts), das bis heute hohe Zustimmung erfährt und in der Literatur zur BNE eine dominante Rolle einnimmt (z.B. Künzli David, Bertschy, de Haan, \& Plesse, 2008). Innerhalb dieses Dreiecks gibt es keine voneinander getrennten Bereiche mehr, sondern sie werden als ein Ganzes betrachtet (Pufé, 2017). Damit werden Prinzipien, Ziele und Regeln verfolgt, die nicht auf eine bestimmte Dimension beschränkt sind (Grünwald \& Kopfmüller, 2012). Ein gleichschenkliges Dreieck veranschaulicht, dass allen drei Seiten eine gleich starke Bedeutung zukommt und ermöglicht es das Prinzip der Nachhaltigkeit relativ gut rechnerisch zu 
operationalisieren (von Hauff \& Kleine, 2009; Pufé, 2017). Eine Überbewertung eines Bereichs bringt das Dreieck aus seiner regelmäßigen Form, indem sich die entsprechende Ecke vom Zentrum entfernt. Damit bietet die Anwendung des Modells die Möglichkeit, nachhaltige Entwicklung durch ein Spinnwebdiagramm sichtbar $\mathrm{zu}$ machen, erlaubt differenzierte Interpretationen und Nachhaltigkeitsbewertung und kann daher auch der Definition von konkreten Handlungsfeldern dienen. Auch in aktuellen Veröffentlichungen, die sich mit Leitbildern nachhaltiger Entwicklung für BNE befassen, werden das integrative Nachhaltigkeitsdreieck oder, ergänzt um die vierte Dimension ,Politik' oder ,Kultur', das Nachhaltigkeitsviereck als zentrale Grundlage beibehalten (Fögele, 2016; KMK \& BMZ, 2016; Keil, 2019).

Kritik an diesen Modellen bezieht sich auf die Auffassung der Gleichwertigkeit aller drei bzw. vier Dimensionen, denen entsprechende Vorrangmodelle entgegengehalten werden (Grünwald \& Kopfmüller, 2012; Knaus \& Renn, 1998). Mit der Begründung, dass es letztlich die natürlichen Bedingungen sind, die die Basis für gesellschaftliches und wirtschaftliches Handeln bilden (Grünwald \& Kopfmüller, 2012; Knaus \& Renn, 1998, vgl. Abb. 3, links), wird in diesen meist der ökologischen Dimension Vorrang eingeräumt. Griggs et al. (2013) rücken in ihrer Nachhaltigkeitskonzeption die sozialen Ziele heutiger und künftiger Generationen ins Zentrum und betonen die Bedeutung fester ökologischer Außengrenzen für das Wirtschaften. Im Modell markieren die AutorInnen die ökologische Dimension als „Earth's life support system, on which the welfare of current and future generations depends" (Griggs et al., 2013, S. 306; vgl. Abb. 3, Mitte). Kritik an diesem Modell bezieht sich vor allem darauf, dass das Bestimmen von ökologischen Grenzen sehr schwierig ist und die Gerechtigkeitsfrage aufgrund der Dringlichkeit der Bearbeitung der ökologischen Krise in den Hintergrund rückt (Grünwald \& Kopfmüller, 2012).

Kapelari, Suzanne (Hg.), Vierte ,Tagung der Fachdidaktik“ 2019:

„Interdisziplinäre fachdidaktische Diskurse zur Bildung für nachhaltige Entwicklung“

(C) 2020 innsbruck university press, ISBN 978-3-99106-019-2, DOI 10.15203/99106-019-2 

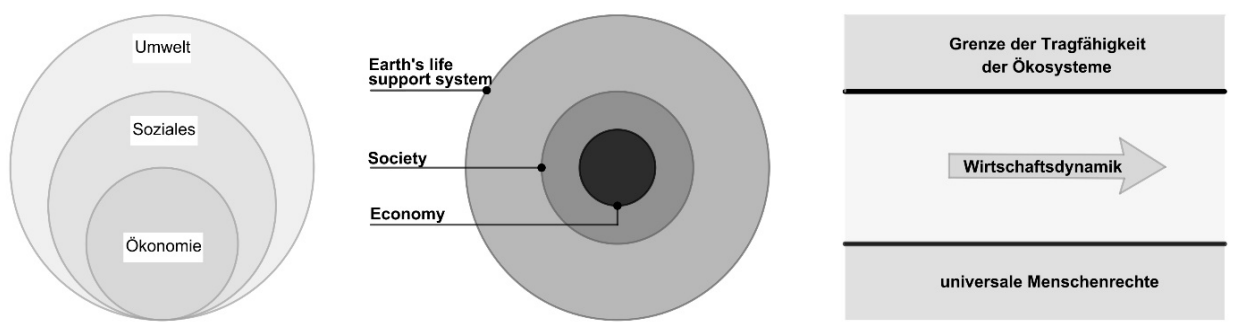

Abb. 3: Vorrang- und Leitplankenmodelle der Nachhaltigkeit (eigene Darstellungen A. M. nach Griggs et al., 2013 und Hoffmann, 2018)

Die ökologische Dimension ist dabei von sog. Leitplanken begrenzt, die die Belastbarkeit der menschlichen Umweltbeeinflussung markieren (Klemmer, Becker-Soest \& Wink, 1998; Grünwald \& Kopfmüller, 2012). Eine Erweiterung dieser Idee wurde im Leitplankenmodell in Abbildung 3 (rechts) dargestellt, indem die Grenze der Tragfähigkeit der Ökosysteme auf der einen Seite um die Grenze der Einhaltung universaler Menschenrechte auf der anderen Seite ergänzt wird (Hoffmann, 2018). Diese Grenzen bilden einen Korridor, in dem wirtschaftliches und soziales Handeln stattfinden darf. Werden die Grenzen überschritten, droht der Zusammenbruch des Systems. Im kritischen Vergleich $\mathrm{zu}$ Mehrdimensionen-Modellen der Nachhaltigkeit sehen auch BNEDidaktikerInnen Stärken in Vorrangmodellen und Leitplankenkonzepten (Niebert, 2016; Bedehäsing \& Padberg, 2017). So wird auch die Dringlichkeit radikaler Umbauten unserer Wirtschaftsweisen angemessener hervorgehoben, was eine wichtige und neue Perspektive darstellt, die aufgrund mangelnden Bezugs zu Degrowth-Konzepten und Suffizienz-Gedanken in der BNE bislang unterrepräsentiert scheint (Getzin \& Singer-Brodowski, 2016; Bedehäsing \& Padberg, 2017).

3.2 Die Donut-Ökonomie als neues Nachhaltigkeitsmodell für den Unterricht

Eine Bestimmung und Operationalisierung der äußeren ökologischen Leitplanke schafft das Stockholm Resilience Centre mit ihrem Konzept der Planetary Boundaries (Rockström et al., 2009; Steffen et al., 2015). Für neun ökologische 
Problemfelder werden Schwellenwerte definiert, die einen konkreten Bereich als sicheren Handlungsraum abstecken, in dem die Menschheit mit hoher Wahrscheinlichkeit langfristig lebensfähig bleibt. An den Grenzen werden Kipppunkte dargestellt, an denen die Menschheit in ökologische Risikobereiche eintritt, in denen mit gravierenden Folgen und irreversiblen Schäden gerechnet werden muss. Hinsichtlich vier von neun Bereichen - Klimawandel, Landnutzungsveränderungen, Biodiversitätsverlust sowie Stickstoff- und Phosphoreintrag in die Biosphäre - wurden die definierten Grenzen bereits überschritten (Rockström et al., 2009; Steffen et al., 2015).

Damit wäre das in Abbildung 3 skizzierte Leitplankenmodell zunächst lediglich im Hinblick auf die ökologische Dimension konkretisiert. Im Modell der DonutÖkonomie von Kate Raworth $(2012 ;$ 2018) werden diesen Leitplanken zusätzlich soziale Mindeststandards gegenübergestellt. Das in diesem Modell definierte soziale Fundament basiert auf zwölf Dimensionen, die aus den von den United Nations [UN] formulierten Zielen für eine nachhaltige Entwicklung (Sustainable Development Goals) (UN, 2015; siehe auch Rieckmann in diesem Band) abgeleitet sind (Raworth, 2018, vgl. Abb. 4 rechts). Zwischen der Abdeckung sozialer Mindeststandards und dem Erreichen der ökologischen Grenzen eröffnet sich ein Handlungsraum, innerhalb dessen ein sicherer und gerechter Lebensraum für alle besteht, ,jener Raum, in dem wir die Bedürfnisse aller mit den Mitteln des Planeten befriedigen können“"(Raworth, 2018, S. 20). Dieser Raum wird im Modell als Donut-förmiger Kreisring zwischen dem inneren Kreis des sozialen Fundaments und dem äußeren Ring der Leitplanken dargestellt. Mit dem Modell versucht die Ökonomin dem wirtschaftlichen Wachstumsnarrativ ein alternatives Denkmodell gegenüberzustellen. Nicht mehr die etablierten, althergebrachten ökonomischen Theorien sollen den Ausgangspunkt ökonomischen Denkens bilden, sondern die langfristigen Ziele der Menschheit. Im Vergleich $\mathrm{zu}$ anderen Konzeptionen nachhaltiger Entwicklung bildet das Donut-Modell ein einfaches Denkwerkzeug und damit einen Interpretationsrahmen, dem die Autorin die Macht zuschreibt, wirtschaftliches Denken im 21. Jahrhundert zu verändern (Raworth, 2018). Aus didaktischer Sicht liegen weitere Stärken in der Zielsetzung der Vermittlung einer positiven Zukunftsvision (Raworth, 2018) und der zentralen Rolle sozioökonomischer Grundbedürfnisse, die als Grundbedingungen menschlichen Wohlbefindens und Aspekte guten Lebens sichtbar werden (vgl. Basiskonzept

Kapelari, Suzanne (Hg.), Vierte „Tagung der Fachdidaktik“ 2019:

„Interdisziplinäre fachdidaktische Diskurse zur Bildung für nachhaltige Entwicklung“

(C) 2020 innsbruck university press, ISBN 978-3-99106-019-2, DOI 10.15203/99106-019-2 
Lebensqualität und Nachhaltigkeit in Kap. 2.2.). Die schlichte Donut-Metapher, die an ein Süßgebäck mit einem Loch in der Mitte erinnert, bildet zudem eine an die Lebenswelt der SchülerInnen anschließende einfache Denk- und Merkfigur (vgl. Abb. 4, links).
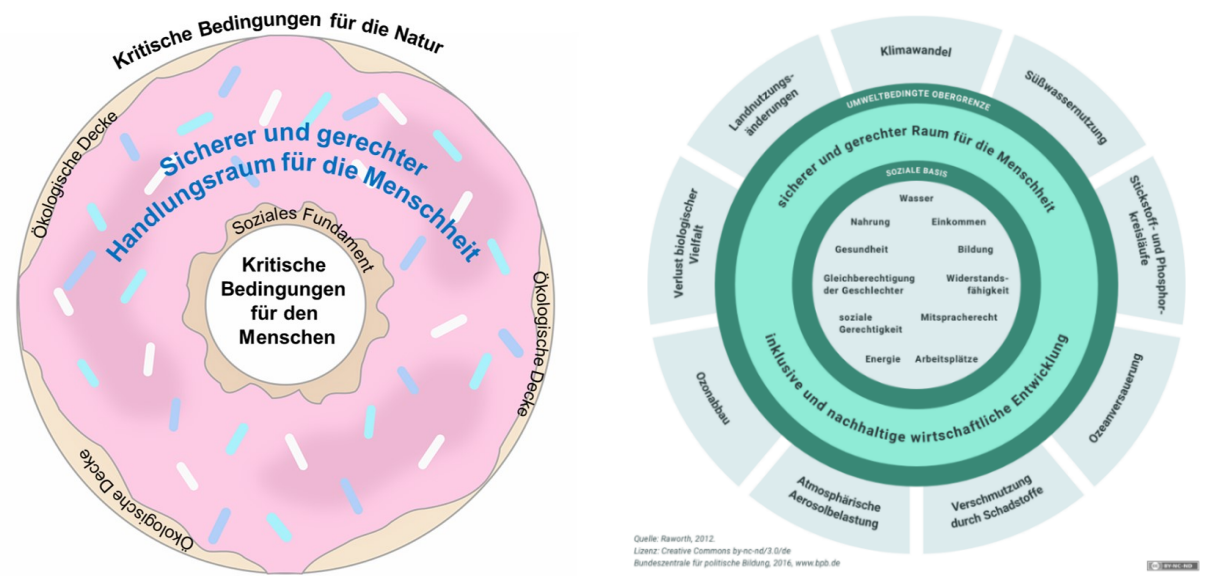

Abb. 4: Das Modell der Donut-Ökonomie (Raworth, 2018) in einfachen Grundzügen (links: eigene Darstellung O. A.) und im Detail (rechts: Bundeszentrale für politische Bildung, 2016)

In Kooperation mit der Universität Leeds (GB) wurde das Modell der DonutÖkonomie in abgewandelter Form $\mathrm{zu}$ einem Monitoring-Projekt weiterentwickelt. Für die Operationalisierung der ökologischen Decke wurden vier der planetaren Belastungsgrenzen herangezogen und um den ökologischen und materiellen Fußabdruck ergänzt. Für das soziale Fundament wurden die ursprünglichen Dimensionen um Indizes der Lebenszufriedenheit ergänzt (O’Neill, Fanning, Lamb, \& Steinberger, 2018). Aufbauend auf diesem Analysemodell wurden, nationale Donuts' für 150 Länder erstellt, die für Ländervergleiche online abgerufen werden können (https://goodlife.leeds.ac.uk/ countries/). Dieses Monitoring-Projekt bildete die Grundlage für die Entwicklung des GeoGebra-Applets, mit dem SchülerInnen das Donut-Modell eigenständig anwenden können (siehe Kap. 4.). Dabei setzen sich die Lernenden mit räumlichen Daten auseinander, bereiten sie mithilfe statistischer Konzepte für eine Integration und Visualisierung im Applet auf und können auf 
unterschiedlichen Maßstabsebenen Herausforderungen und Handlungswege nachhaltiger Entwicklung erkennen.

Die nationalen Donuts zeigen, dass Länder wie Österreich oder Deutschland Vorgaben bezüglich sozialer Absicherung oft zur Gänze erreichen, gleichzeitig aber die biophysischen Grenzen stark strapazieren. Ärmere Länder bleiben hingegen innerhalb dieser biophysischen Grenzen, erfüllen die sozialen Mindeststandards aber kaum (vgl. Abb. 5). Dass keines der 150 untersuchten Länder der Erde die Vorgaben der Donut-Ökonomie hinsichtlich nachhaltiger Entwicklung erfüllt, also alle sozialen Mindeststandards sichert, ohne die ökologischen Belastungsgrenzen zu überschreiten (O’Neill, Fanning, Lamb, \& Steinberger, 2018), demonstriert die dringende Notwendigkeit einer großen Transformation hin zu neuen, nachhaltigen Wirtschaftsweisen. In diesem Sinne dient das Analysieren und Visualisieren nachhaltiger Entwicklung mittels des Donut-Modells im Unterricht auch dazu, das westliche Entwicklungsideal in Frage zu stellen und zu erkennen, dass auch das eigene Land (z.B. Österreich) hinsichtlich des Erreichens nachhaltiger Entwicklungsziele ein Entwicklungsland ist (Holzinger, 2019; siehe auch Rieckmann in diesem Band).

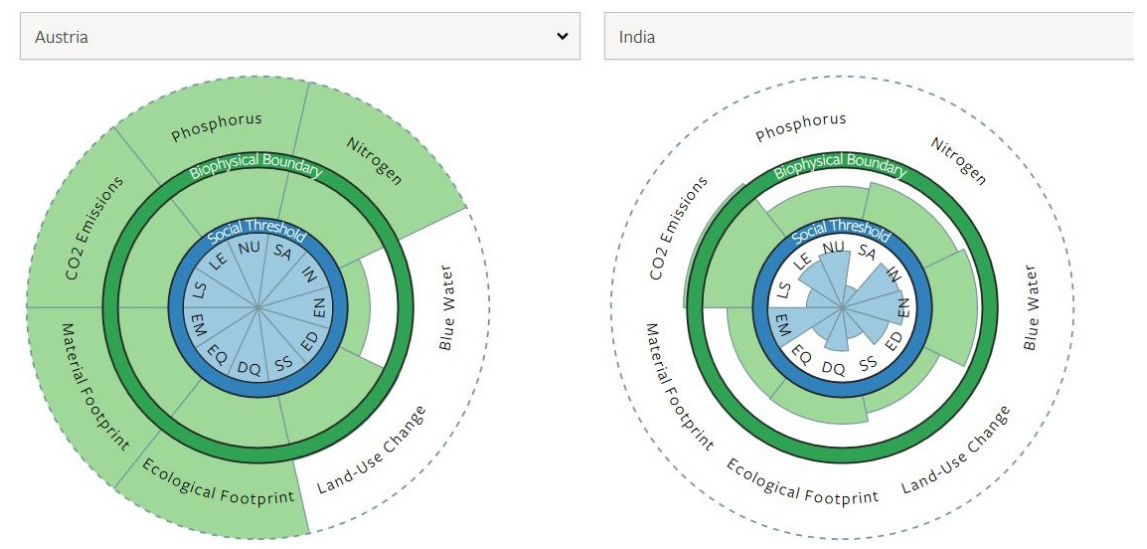

Abb. 5: Donuts von Österreich und Indien im direkten Vergleich (https://goodlife.leeds.ac.uk/countries/, 05.06.2019)

LS - Life Satisfaction/Lebenszufriedenheit, LE - Healthy Life Expectancy/Lebenserwartung, NU - Nutrition/Ernährung, SA - Sanitation/Wasser \& Hygiene, IN - Income/Einkommen, EN Access to Energy/Zugang zu Energie, ED - Education/Bildung, SS - Social Support/ Sozialleistungen, DQ - Democratic Quality/Demokratiequalität, EQ - Equality/Gleichheit, EM Employment/Beschäftigung. 
Das Modell eröffnet somit eine konstruktive, problem- und lösungsorientierte Möglichkeit zur Auseinandersetzung mit dem Leitbild nachhaltiger Entwicklung. Wie es Kompetenzen für emanzipatorische BNE vorsehen, kann damit nachhaltige Entwicklung als ergebnisoffener, gesellschaftlicher Lernprozess sichtbar werden (Vare \& Scott, 2007), in dem die Erarbeitung von Lösungen als Such- und Orientierungsbewegungen innerhalb des DonutRahmens transparent werden. Durch einen Transfer zwischen den Maßstabsbzw. Handlungsebenen können diese Orientierungsbewegungen von SchülerInnen auch auf individueller Ebene mit der eigenen Lebenswelt verbunden werden. Anhand des Donut-Modells können die Beziehungen zwischen den Vorstellungen von guter Lebensqualität und damit verbunden mit der Gestaltung des alltäglichen Lebens auf der einen Seite, zu den ökologischen, sozialen, wirtschaftlichen Bedingungen und Auswirkungen auf der anderen Seite, greifbar gemacht werden und dadurch die Entwicklung von Urteils-, Reflexions- und Handlungskompetenzen der SchülerInnen stärken.

\section{Beschreibung der Lernumgebung und projektbegleitende Evaluation}

Im Folgenden wird das Projekt ${ }^{2}$ One Donut for all über die einzelnen Projektphasen und die Erhebung von Daten näher beschrieben. Die grundlegende Idee des Projektes besteht darin, eine fächerübergreifende Lernumgebung für den Mathematik- und GW-Unterricht der Sekundarstufe $1 \mathrm{zu}$ entwickeln, welche mit einem datenbasierten Zugang zur BNE die Entwicklung von Kompetenzen in den Bereichen Statistik und GW sowie die Entwicklung überfachlicher Kompetenzen (vgl. Kap. 2.) fördert. Die Forschungsfragen, die den Leitfaden für die Evaluation und auch die Entwicklung des Unterrichtsentwurfs bilden, betreffen einerseits den Kontext der nachhaltigen Entwicklung im Sekundarstufenunterricht und andererseits die Kompetenzentwicklung bei SchülerInnen aus der Sicht der beiden Unterrichtsfächer. Die Durchführung in zwei Klassen der 8. Schulstufe fand im April und Mai des Schuljahres 2018/19 statt. Nach Absprache mit den

2 Das verwendete GeoGebra-Applet sowie die zugehörigen Arbeitsblätter sind als Open Educational Ressource unter https:/www.geogebra.org/m/tythjgw9 erhältlich.

Kapelari, Suzanne (Hg.), Vierte „Tagung der Fachdidaktik“ 2019:

„Interdisziplinäre fachdidaktische Diskurse zur Bildung für nachhaltige Entwicklung“

(C) 2020 innsbruck university press, ISBN 978-3-99106-019-2, DOI 10.15203/99106-019-2 
Klassenlehrern wurde die Erprobung des Unterrichtsentwurfs von den ProjektleiterInnen als Lehrpersonen durchgeführt. Die einzelnen Projektphasen fanden meist in heterogenen Kleingruppen statt, welche von den Klassenlehrern zusammengestellt wurden. Konkrete Ergebnisse der projektbegleitenden Evaluation liegen noch nicht vor. Aus ersten Auswertungen der durch die Arbeitsergebnisse der SchülerInnen und durch Fragebögen, Audio- und Videographie gewonnen Daten sowie aus den Erfahrungen in der Umsetzung lassen sich jedoch Eindrücke und erste Ergebnisse ableiten, die wir in die folgende Beschreibung des Projektablaufs einfließen lassen.

Das gesamte Projekt wurde über einen Zeitraum von zwei Wochen in Einzeloder Doppelstunden durchgeführt und in vier Phasen von der Einführung zum Thema und des Donut-Modells über die Anwendung des Modells bis hin zur Abschlussphase aufgebaut (vgl. Abb. 6).

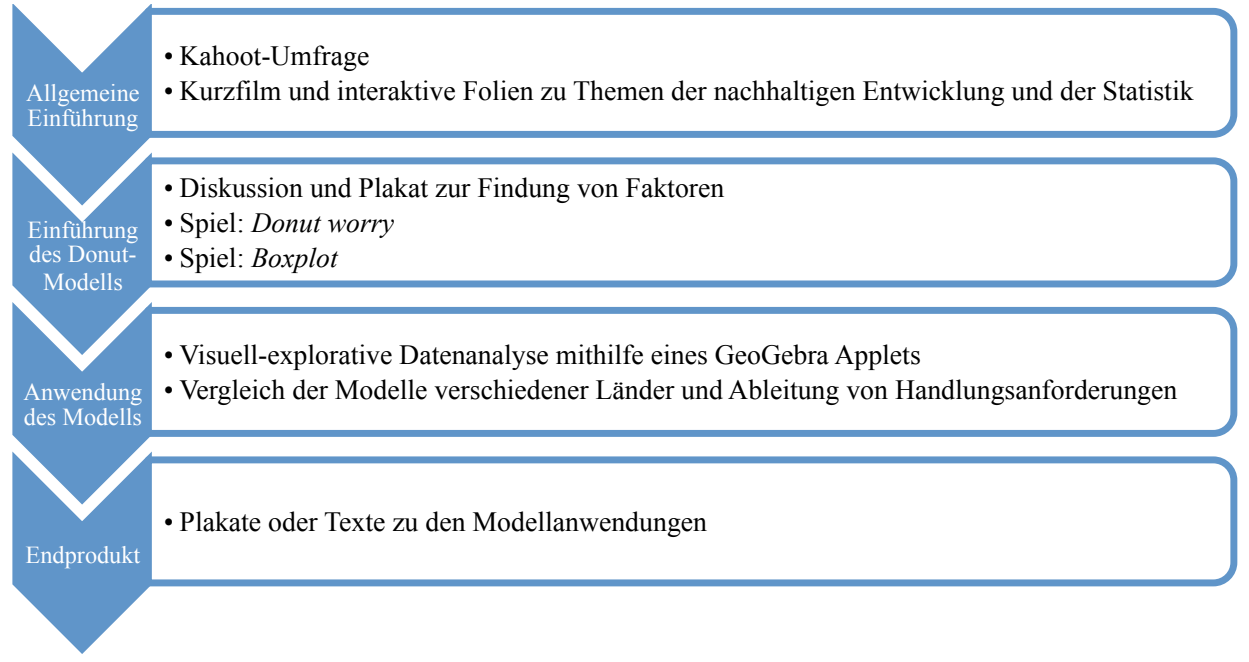

Abb. 6: Chronologische Darstellung der Projektphasen

Nach einer kurzen Information zum Ablauf und zur Evaluierung des Projekts nahmen die SchülerInnen als Einstieg an einer Umfrage nach dem Vorbild der Testfragen zu Gapminder's Ignorance Project (Rosling et al., 2018) teil. Die Umfrage umfasst Fragen zum Entwicklungsstand der Welt, wie etwa konkret 
zur weltweiten Masernimpfrate, zur Entwicklung extremer Armut oder zur weltweit durchschnittlichen Dauer des Schulbesuchs. Die SchülerInnen hatten jeweils eine Minute Zeit, die Frage in den heterogenen Kleingruppen zu diskutieren und die ausgewählte Antwort einzuloggen. Die (richtigen) Antworten sowie die Bearbeitungszeit wurden digital erfasst und mittels eines automatischen Rankings rückgemeldet. Die Ergebnisse dieser Umfrage zeigen, dass die SchülerInnen mit etwa einem Drittel richtiger Antworten rund um den Erwartungswert einer zufälligen Auswahl liegen und damit jedoch weniger vorurteilsbehaftet antworteten als der weltweite Durchschnitt bei dieser Umfrage (Rosling et al., 2018). Einzig die Fragen zum Artenschutz, der sich in den letzten Jahrzehnten verbessert hat, und $\mathrm{zu}$ den Sterbefällen durch Naturkatastrophen, die sich im letzten Jahrhundert halbiert haben, liegen aufgrund der nur einzeln oder gar nicht gegebenen, richtigen Antworten unter dem Durchschnitt.

Zur tiefergehenden Beantwortung der Fragen über die Entwicklung der Welt wurde anschließend an die Umfrage der 20-minütige Film „How not to be ignorant about the world“ (Rosling \& Rosling, 2014) gezeigt. Darin berichtet der schwedische Entwickler des Gapminder Projekts, Hans Rosling, anhand von Auswertungen der Antworten zu dieser Umfrage, die in verschiedenen Nationen mit unterschiedlichem Publikum abgehalten wurde, dass viele Menschen weltweit $\mathrm{zu}$ wenig über globale und nachhaltige Entwicklung wissen und informiert sind bzw. in ihren Antworten von Vorurteilen geleitet sind (Rosling et al., 2018). In Gruppendiskussionen wurde dann reflektiert, welche diesbezüglichen Vorstellungen und persönliche Vorurteile die SchülerInnen bei sich selbst sehen, wie diese entstehen und sie in ihrem Denken und Handeln beeinflussen.

Als letzter Teil in der Einführungsphase wurde den SchülerInnen mittels interaktiven Folien zur Entwicklung extremer Armut auf der Welt (https://www.gapminder.org/downloads/human-development-trends-2005/) sowie einem begleitenden Arbeitsblatt die Visualisierungssoftware von Gapminder nähergebracht. Nach der Bearbeitung des Arbeitsblatts in den Kleingruppen wurden die Ergebnisse im Plenum verglichen. Durch qualitative Analyse der Arbeitsblätter soll in einem weiteren Schritt erörtert werden, welchen Beitrag die interaktiven Folien als Hinführung zur Software und zu den

Kapelari, Suzanne (Hg.), Vierte „Tagung der Fachdidaktik“ 2019:

„Interdisziplinäre fachdidaktische Diskurse zur Bildung für nachhaltige Entwicklung“

(C) 2020 innsbruck university press, ISBN 978-3-99106-019-2, DOI 10.15203/99106-019-2 
statistischen Inhalten bei der Kompetenzentwicklung hinsichtlich der visuellexplorativen Datenanalyse leisten können.

Das Konzept der Donut-Ökonomie, welches als Grundlage für den weiteren Projektverlauf diente, wurde den SchülerInnen zunächst mit unterschiedlichen, meist spielerischen Methoden nähergebracht. Um jene Faktoren des Modells zu erarbeiten, die für die SchülerInnen von Relevanz sind (vgl. Kap. 2.2.), wurde zu Beginn gemeinsam erarbeitet, was für sie ein gutes Leben ausmacht, was in ihrem Alltag von großer Bedeutung ist und welche Auswirkungen dies auf Umwelt und Ressourcen der Erde hat (vgl. Abb. 7). Um die SchülerInnen für die Unterscheidung zwischen sozialen Grundbedürfnissen und biophysischen Grenzen zu sensibilisieren, wurden Post-its mit Stichworten zu den Ergebnissen dieser Arbeit auf einem Plakat mit einem skizzierten Donut platziert. In Abbildung 7 kann man erkennen, dass den SchülerInnen vor allem Handys, Autos, Wasser-, Nahrungsmittel- und Energieversorgung als unverzichtbare Aspekte ihres Alltages erscheinen. Auch wurden einige Auswirkungen auf die natürliche Umwelt der Erde, wie Abgase und Luftverschmutzung, Massentierhaltung oder Rodung genannt.

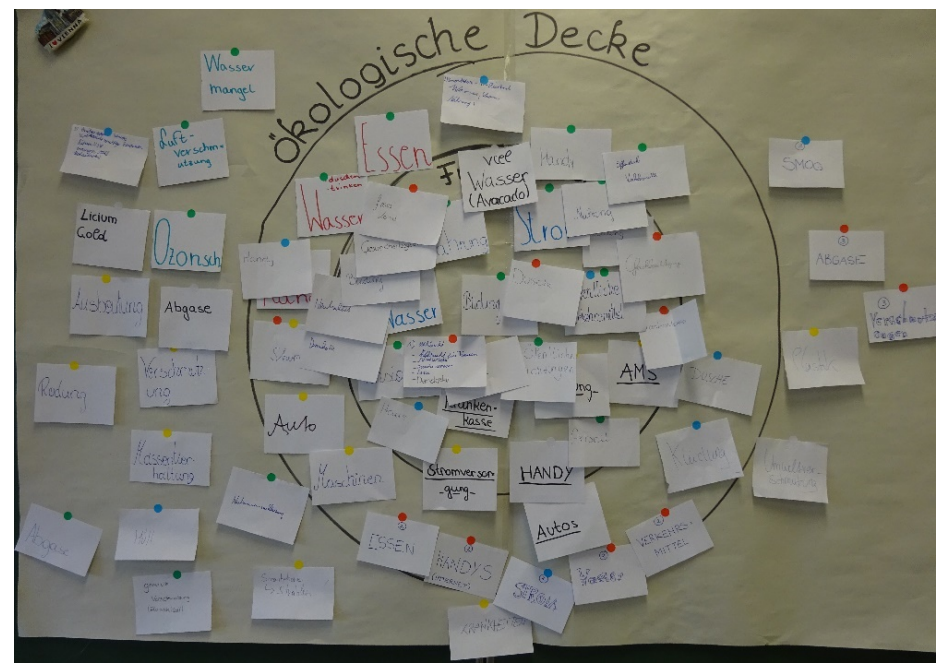

Abb. 7: Zuordnung von - für die Lernenden relevanten - Faktoren im Donut-Modell (Eigene Darstellung A. M.) 
Um das Konzept der Donut-Ökonomie weiter zu vertiefen und die Beziehungen der einzelnen Faktoren aufzuzeigen, wurde das vorab entwickelte Brettspiel Donut worry eingesetzt (siehe Abb. 8). Die Spielkarten beinhalten Informationen zu ausgewählten sozio-ökonomischen und ökologischen Faktoren. Ziel des Spiels ist es sich entsprechend der gewürfelten Augenzahl strategisch sinnvoll für einen sozioökonomischen oder ökologischen Faktor zu entscheiden, um in den grünen Ring zu gelangen bzw. sich möglichst wenig weit davon zu entfernen.

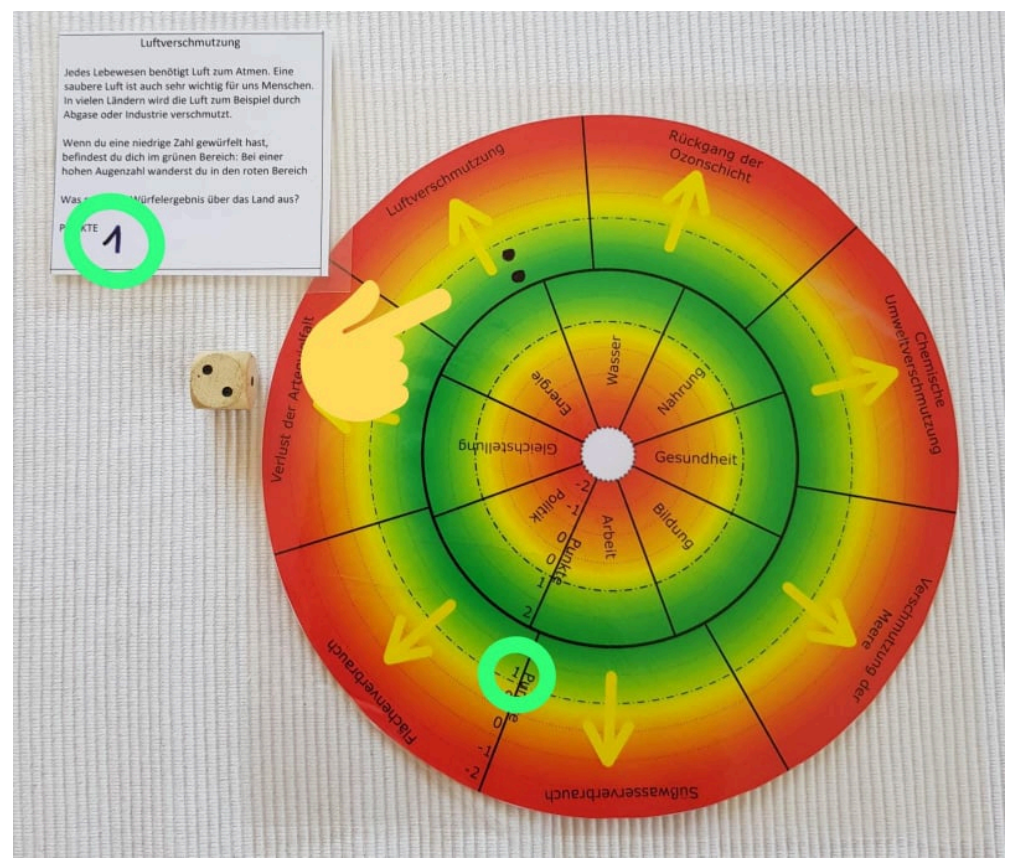

Abb. 8: Auszug aus der Spielanleitung zum Brettspiel Donut worry (Eigene Darstellung Z. M.)

Ein zweites Spiel, das Kartenspiel Duett, diente vor allem der Zuordnung zwischen Informationen, die einerseits in Textform und Boxplots und andererseits als Punktwolkendiagramme bzw. Bubblecharts gegeben sind (siehe Abb. 9). Das Lernziel hinter diesem Spiel ist, verschiedene Darstellungsformen von Informationen miteinander $\mathrm{zu}$ verbinden, indem das intuitive Wissen $\mathrm{zu}$ statistischen Ideen (Garfield \& Ben-Zvi, 2008) angewandt wird. Erste 
Ergebnisse lassen erkennen, dass die SchülerInnen durchwegs die im Text des Kartenspiels angesprochenen, statistischen Ideen, wie etwa die Idee von Zentralwerten, Streuung oder Korrelation, mit den graphischen Darstellungsformen verbinden konnten. Die Zuordnung der dargestellten Faktoren zu den sozialen Grundbedürfnissen bzw. den biophysischen Grenzen inspirierte mitunter auch spannende Diskussionen. So wurde z.B. der Faktor ,Kinder pro Frau' kontrovers interpretiert und in den Gruppen unterschiedlich zugeordnet. Dabei war die Vorstellung, dies als Faktor des sozialen Fundaments, etwa hinsichtlich der Selbstbestimmung von Frauen, zu sehen, dominanter als die Tendenz, den Faktor der ökologischen Dimension und Problemfeldern, die aus Bevölkerungswachstum und Überbevölkerung resultieren, zuzuordnen.
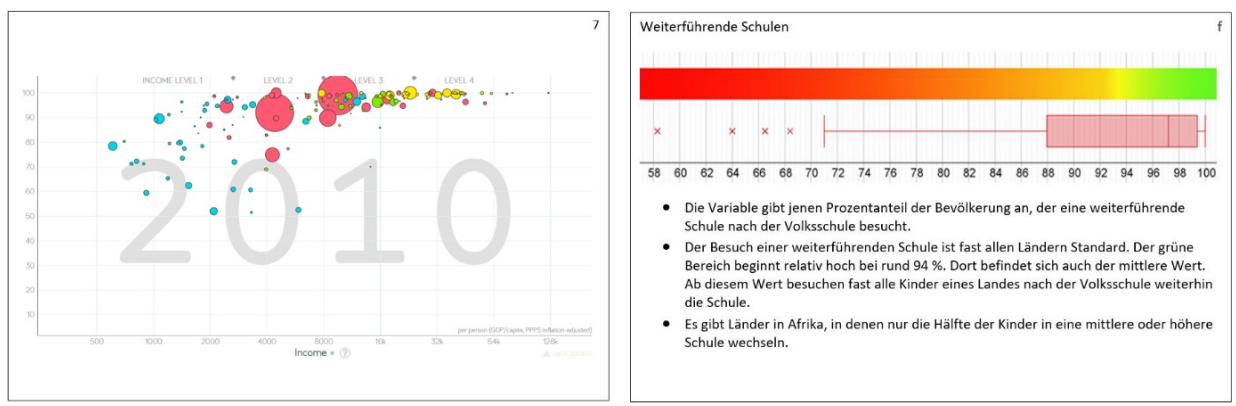

Abb. 9: Kartenpaar des Kartenspiels Duett mit Informationen in verschiedenen Darstellungsformen basierend auf den Abbildungen und Daten von www.gapminder.org (Eigene Darstellung A. M.)

In der nun im Folgenden beschriebenen, dritten Projektphase stand der visuellexplorative Forschungskreislauf im Zentrum. Die SchülerInnen arbeiteten dabei selbständig mit den Daten und Visualisierungen der Software Gapminder und einem speziell für dieses Projekt entwickelten GeoGebra-Applet (vgl. Abb. 10, rechts).

In Kleingruppen wandten die SchülerInnen das Donut-Modell auf ausgewählte Staaten an. Die zu bearbeitenden Faktoren wurden von den SchülerInnen anhand einer Liste der in Gapminder verfügbaren Daten selbst gewählt. Durch intuitives Erkennen und Anwenden von statistischen Ideen, die durch

Kapelari, Suzanne (Hg.), Vierte „Tagung der Fachdidaktik“ 2019:

„Interdisziplinäre fachdidaktische Diskurse zur Bildung für nachhaltige Entwicklung“

(C) 2020 innsbruck university press, ISBN 978-3-99106-019-2, DOI 10.15203/99106-019-2 
unterschiedliche Visualisierungsformen der Daten befördert werden (Andre et al., 2019), und das Durchlaufen mehrerer Kreisläufe der visuell-explorativen Datenanalyse (Prodromou, 2014) erstellten die SchülerInnen mithilfe des GeoGebra-Applets einen nationalen Donut. Dabei wurden die Werte aus den graphischen Darstellungen der Software Gapminder hinsichtlich ihrer Wertigkeit im Sinne der nachhaltigen Entwicklung interpretiert und mittels Schieberegler im Donut-Applet abgebildet. Um diesen Schritt zu erleichtern, wurde für einige Variablen eine Farbskala erstellt, die in Übereinstimmung mit den planetary boundaries (Steffen et al., 2015) Aufschluss über die Bedeutung der abgelesenen Werte für die nachhaltige Entwicklung gibt (vgl. Abb. 10, rechts). Bei Variablen ohne vorgegebene Farbskala diskutierten die SchülerInnen in den Kleingruppen über die Bedeutung des Faktors für die nachhaltige Entwicklung des Landes und legten selbst Grenzwerte fest. Dabei wurden u.a. die Ideen der Zentralwerte oder der Streuung als Begründung für die Anwendung und farbliche Zuordnung der Daten im Donut-Modell herangezogen. Die Ergebnisse dieser Arbeitsschritte wurden für jeden Faktor in einem Arbeitsblatt dokumentiert (vgl. Abb. 10, links).

Am Ende dieser Phase wurden die Ergebnisse der Arbeit der Kleingruppen im Plenum vorgestellt und die Übereinstimmungen und Abweichungen in den Visualisierungen der nationalen Donuts mit jenen nationalen Donuts, die von Forschenden der University of Leeds erstellt worden sind (vgl. Kap. 3.2., Abb. 5), analysiert. Erste Ergebnisse der Auswertungen des Projekts zeigen eine gute inhaltliche Qualität und Plausibilität der von den SchülerInnen begründet erstellten, nationalen Donuts. In der an das Projekt anschließenden Fragebogenerhebung zeigte die Mehrheit der SchülerInnen, dass sie durch die Arbeit mit dem Donut-Modell Zielsetzungen nachhaltiger Entwicklung verstanden haben. Sie konnten einen gegebenen, unbeschrifteten, nationalen Donut hinsichtlich der nachhaltigen Entwicklung eines (fiktiven) Staates interpretieren und generelle Aussagen zur Entwicklung des Landes ableiten. Weiters wurde von den SchülerInnen die Möglichkeit des selbstgesteuerten, explorativen Lernprozesses als positive Erfahrung hervorgehoben. 


\begin{tabular}{|l|l|l|l|}
\hline Variablen-name & $\begin{array}{l}\text { Sozialer } \\
\text { oder } \\
\text { okologischer } \\
\text { Faktor? }\end{array}$ & $\begin{array}{l}\text { Zahlenwert } \\
\text { der Variable }\end{array}$ & \multicolumn{1}{|c|}{ Begründung für die Klassifizierung (Farbwahll) } \\
\hline Oo ${ }^{2}$ & ÖU & $7,4 \mathrm{t}$ & Ist zu viel /person \\
\hline $\begin{array}{l}\text { Threatend } \\
\text { Species }\end{array}$ & Ö/U & $\begin{array}{l}100,3 \\
\text { Tiere }\end{array}$ & Zu hohe Anzahl \\
\hline $\begin{array}{l}\text { Threatend } \\
\text { plant }\end{array}$ & Ö/U & $\begin{array}{l}574 \\
\text { Pflanzen }\end{array}$ & Zu hohe Zahl \\
\hline $\begin{array}{l}\text { Babys } \\
\text { /woman }\end{array}$ & S/W & $\begin{array}{l}1,64 \\
\text { Babys }\end{array}$ & $\begin{array}{l}\text { Guter Durchschnitt (hält die } \\
\text { Bevölkerung) }\end{array}$ \\
\hline $\begin{array}{l}\text { Child } \\
\text { Mortality }\end{array}$ & S/W & $\begin{array}{l}9,95 \\
\text { Kinder }\end{array}$ & $\begin{array}{l}\text { Von 1000 Kindern 10 tote ist nicht } \\
\text { sehr viel }\end{array}$ \\
\hline $\begin{array}{l}\text { Democracy } \\
\text { Score }\end{array}$ & S/W & -7 & Die Regierung bestimmt viel zu viel \\
\hline $\begin{array}{l}\text { Working } \\
\text { Hours } \\
\text { /week }\end{array}$ & S/W & $46,2 \mathrm{~h}$ & $\begin{array}{l}\text { Zu viel, aber immer noch besser als } \\
\text { keine Arbeit }\end{array}$ \\
\hline $\begin{array}{l}\text { Water } \\
\text { Withdrawal }\end{array}$ & Ö/U & $430 \mathrm{~m}^{3}$ & $\begin{array}{l}\text { Bisschen unter dem Median, aber } \\
\text { trz. nicht zu viel }\end{array}$ \\
\hline $\begin{array}{l}\text { At least } \\
\text { basic water } \\
\text { source }\end{array}$ & O/U & $90,8 \%$ & $\begin{array}{l}\text { Das ca. } \text { 90\% der Leute zugang zu } \\
\text { Wasser haben ist nicht so schlecht. }\end{array}$ \\
\hline
\end{tabular}

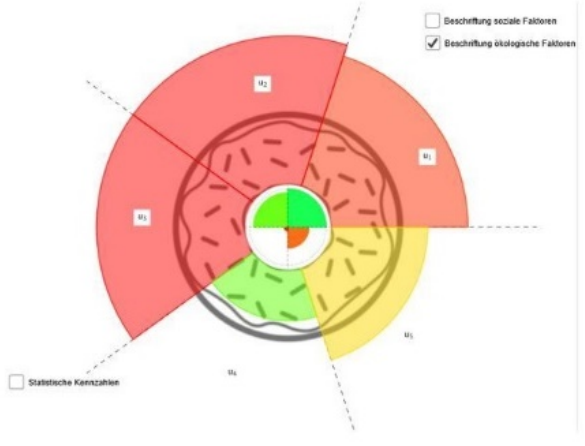

Abb. 10: Arbeitsblatt einer Kleingruppe zur Erstellung des nationalen Donuts und GeoGebraDonut

In der letzten Phase des Projekts wurden in beiden Klassen zwei unterschiedliche Methoden zur Synthese und Reflexion der Inhalte ausgewählt. In einer der beiden Klassen lautete der Arbeitsauftrag an die Kleingruppen, sich gedanklich in eine/n BewohnerIn des Landes, das zuvor bearbeitet wurde, hineinzuversetzen und anhand des entwickelten Donuts und von Bildern der Bilderdatenbank Dollarstreet einen Text über die Lebenssituation und den Alltag diesen/dieser Bewohners/Bewohnerin in Ich-Perspektive zu verfassen. Dabei griffen die SchülerInnen durchwegs die zuvor bearbeiteten Faktoren und Variablen auf und fokussierten in ihrer verbalen Bewertung auch auf jene Bereiche mit Entwicklungspotenzialen hinsichtlich der nachhaltigen Entwicklung dieser Länder. Die Ergebnisse geben Anlass zur Vermutung, dass die Umsetzung des statistical storytelling (Skranefjell \& Tønnessen, 2003) in Verbindung mit relevanten Kontexten (Makar \& Ben-Zvi, 2011) zu einem vertiefenden Kompetenzerwerb im Statistikunterricht der Sekundarstufe I beitragen kann.

In der zweiten der beiden Klassen lautete der Arbeitsauftrag an die Kleingruppen, ein Poster zum zuvor erarbeiteten Land zu erstellen, das über einen Faktor informiert, welcher hinsichtlich der nachhaltigen Entwicklung in diesem Land besonders bedeutend ist. Sie entwickelten dazu passende

Kapelari, Suzanne (Hg.), Vierte „Tagung der Fachdidaktik“ 2019:

„Interdisziplinäre fachdidaktische Diskurse zur Bildung für nachhaltige Entwicklung“

(C) 2020 innsbruck university press, ISBN 978-3-99106-019-2, DOI 10.15203/99106-019-2 
Handlungsanforderungen für die zukünftige Entwicklung und präsentierten diese in einem digital erstellten Poster (vgl. Abb. 11).

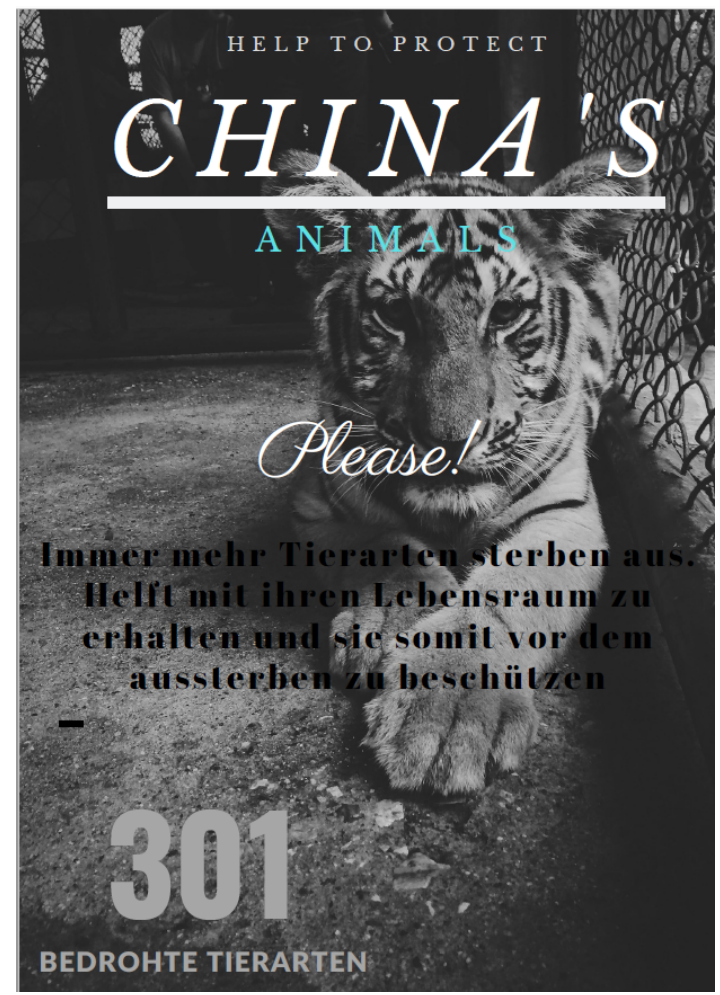

Abb. 11: Poster einer Kleingruppe über bedrohte Tierarten (Biodiversität) in China

Einerseits sollte mit der Erstellung und der Diskussion der nationalen Donuts und der Poster im Plenum die kritische Reflexion $\mathrm{zu}$ möglichen Handlungsfeldern hinsichtlich der nachhaltigen Entwicklung der bearbeiteten Staaten ermöglicht und ein Überblick über die unterschiedlichen Zielkonflikte und Anforderungen nachhaltiger Entwicklungswege gegeben werden. Dabei zeigte sich den SchülerInnen, dass auch Länder des globalen Nordens (z.B. Österreich) als Entwicklungsländer hin zu einer zukunftsfähigeren Entwicklung bezeichnet werden können. Andererseits könnte die komprimierte Darstellung von Informationen in einer vereinfachten, ersten Form einer Infografik die 
Entwicklung von statistischen Kompetenzen hinsichtlich der Darstellung und Bewertung von Information unterstützen.

Erste Auswertungen der erhobenen Projektdaten zeigen, dass der Prozess der Auswahl von Faktoren und passender Darstellungsformen hin zur digitalen Umsetzung von den Kleingruppen oft als schwierig empfunden wurde. Die Lernziele der kritischen Reflexion der dargestellten Entwicklungen und der Herstellung von Verbindungen zur eigenen Lebenswelt konnten weitgehend erreicht werden.

\section{$5 \quad$ Fazit und Ausblick}

Anhand der in diesem Beitrag dargestellten Lernumgebung soll SchülerInnen ein datenbasierter Zugang zur BNE eröffnet werden. Neben zentralen Inhalten und Kompetenzen in den Fächern Mathematik und GW werden auch weitere Kompetenzbereiche, wie jene der Digitalen Grundbildung, angesprochen. Das für dieses Projekt erstellte, interaktive Applet zusammen mit dem Arbeitsmaterial ermöglicht es SchülerInnen, in eigenständiger Arbeit die Qualität der nachhaltigen Entwicklung von Ländern, Staatengemeinschaften oder Kontinenten visuell $\mathrm{zu}$ erforschen. Intuitive Zugänge $\mathrm{zu}$ statistischen Grundideen (Andre et al., 2019) gebunden an den Kontext (Makar \& Ben-Zvi, 2011) der nachhaltigen Entwicklung und das Konzept der visuell-explorativen Datenanalyse (Prodromou, 2014) bilden die Grundidee hinter der Arbeit mit dieser Anwendung. Die dabei verwendeten und erstellten Grafiken bilden als Zwischenergebnis wiederum einen Ausgangspunkt für weitere Denk-, Reflexions- und Formalisierungsprozesse hinsichtlich der Lernziele in beiden Fächern.

Die anstehende Auswertung der erhobenen qualitativen Daten soll Aufschluss über die Qualität der Lernprozesse in den Bereichen der Statistik und der BNE geben und kann zur Generierung von tragfesten Hypothesen über die beschriebenen Lernprozesse beitragen, sodass weitere Forschungsarbeiten auch im quantitativen Bereich möglich werden.

Kapelari, Suzanne (Hg.), Vierte „Tagung der Fachdidaktik“ 2019:

„Interdisziplinäre fachdidaktische Diskurse zur Bildung für nachhaltige Entwicklung“

(C) 2020 innsbruck university press, ISBN 978-3-99106-019-2, DOI 10.15203/99106-019-2 
Darüber hinaus ist eine Adaption des Projekts für verschiedene Bereiche angedacht. Speziell durch die Verwendung der Bilderdatenbank Dollarstreet ist es möglich, junge Lernende schon in der Primarstufe für die Anliegen der BNE zu gewinnen, während die Entwicklung statistischer Ideen über einfache visuellstatistische Arbeitsweisen gefördert wird. Weiters können durch eine Adaption des Projekts auch statistische Inhalte in der Sekundarstufe, wie etwa Verteilungen oder auch weiterführende Hypothesentests, aufgegriffen werden. Auch eine Adaptierung und Vertiefungen in Richtung anderer Schultypen bzw. Schulfächer wäre denkbar, sodass beispielsweise in wirtschaftlichen Schulen die nachhaltige Entwicklung von Betrieben oder in Gastronomiefachschulen Lebensmittel hinsichtlich ihrer nachhaltigen Produktion nach dem Vorbild bekannter Fair-Trade Marken untersucht werden können.

Mittels des Unterrichtsbeispiels kann ein weiterer Baustein für interdisziplinäre bzw. fachübergreifende Ansätze zur Verankerung von BNE im Schulunterricht geliefert werden. BNE als generelles Unterrichtsprinzip soll kritische BürgerInnen bilden, die emanzipiert an der Gestaltung der Zukunft teilhaben können und Handlungswege mitentwickeln, die innerhalb des sicheren und gerechten Raumes für menschliche Entwicklung verlaufen. Das Modell der Donut-Ökonomie kann diesen Prozess einen Analyse-, Interpretations- und Diskussionsrahmen geben, der nicht zuletzt auch auf individueller Ebene eine konstruktive Auseinandersetzung mit Bedürfnissen, Zielkonflikten und Handlungsoptionen möglich macht.

\section{Literatur}

Andre, M., Lavicza, Z., \& Prodromou, T. (2019). Die Relevanz von Armut: Kritisches Denken durch Visualisierung sozial- und wirtschaftspolitischer Daten mit Gapminder entwickeln [The relevance of poverty: A project to develop critical thinking by visualizing social and economic data with Gapminder]. Transfer Forschung <-> Schule, 5, 139-147.

Andre, M., Lavicza, Z., \& Prodromou, T. (Feber, 2019). Formalizing students' informal statistical reasoning on real data: Using Gapminder to follow the cycle of inquiry and visual analyses. Konferenzbeitrag am Eleventh Congress of the European Society for Research in Mathematics Education, Utrecht, Niederlande.

Kapelari, Suzanne (Hg.), Vierte „Tagung der Fachdidaktik“ 2019:

„Interdisziplinäre fachdidaktische Diskurse zur Bildung für nachhaltige Entwicklung“

(C) 2020 innsbruck university press, ISBN 978-3-99106-019-2, DOI 10.15203/99106-019-2 
Bagoly-Simó, P. (2014). Implementierung von BNE am Ende der UN-Dekade. Eine internationale Vergleichsstudie am Beispiel des Fachunterrichts. Zeitschrift für Geographiedidaktik - Journal of Geography Education, 42(4), 221-256.

Barth, M., Godeman, J., Rieckmann, M., \& Stoltenberg, U. (2007). Developing key competencies for sustainable development in higher education. International Journal of Sustainability in Higher Education, 8(4), 416-430. doi:10.1108/14676370710823582

Bedehäsing, J., \& Padberg, S. (2017). Globale Krise, Große Transformation, Change Agents: Heiße Eisen für die Geographiedidaktik? GW-Unterricht, 146, 19-31. doi:10.1553/gw-unterricht146s 19

Ben-Zvi, D., \& Aridor-Berger, K. (2016). Children's Wonder How to Wander Between Data and Context. In D. Ben-Zvi, \& K. Makar (Eds.), The Teaching and Learning of Statistics (pp. 25-36). Cham: Springer. doi:10.1007/978-3-319-23470-0 3

Biehler, R., Ben-Zvi, D., Bakker, A., \& Makar, K. (2012). Technology for Enhancing Statistical Reasoning at the School Level. In M. A. Clements, A. J. Bishop, C. Keitel, J. Kilpatrick, \& F. K. S. Leung (Eds.), Third International Handbook of Mathematics Education (pp. 643-689). New York: Springer. doi:10.1007/978-1-4614-4684-2_21

Biehler, R., Kurtzmann, G., \& Prömmel, A. (Hrsg.). (2019). Stochastik in der Schule, $39(1)$.

Bundesländerkonferenz [BLK]. (2003). Orientierungshilfen für die Erstellung einer Präambel und Empfehlungen/Richtlinien zur "Bildung für nachhaltige Entwicklung" an allgemeinbildenden Schulen. Berlin. Verfügbar unter http://www.transfer-21.de/daten/texte/Praeambel-Richtlinien.pdf

Brockmüller, S., Volz, D., \& Siegmund, A. (2016). Der Einsatz experimenteller Arbeitsweisen zur Förderung geographischen Systemverständnisses bei Schüler/innen und Lehramtsstudierenden. In K.-H. Otto (Hrsg.), Geographiedidaktische Forschungen: Vol. 63. Geographie und naturwissenschaftliche Bildung - Der Beitrag des Faches für Schule, Lernlabor und Hochschule: Dokumentation des 21. HGD-Symposiums im März 2015 in Bochum (S. 104-123). Münster: Verlagshaus Monsenstein und Vannerdat OHG.

Bundesministerium für Bildung und Frauen [BMBF]. (2012). Die kompetenzorientierte Reifeprüfung aus Geographie und Wirtschaftskunde. Richtlinien und Beispiele für Themenpool und Prüfungsaufgaben. Wien. Verfügbar unter http://www.bmukk.gv.at/medienpool/22201/reifepruefung ahs lfgw.pdf

Bundesministerium für Bildung und Frauen [BMBF]. (2014). Grundsatzerlass Umweltbildung für nachhaltige Entwicklung. Wien. Verfügbar unter https://bildung.bmbwf.gv.at/ministerium/rs/2014 20 ge umwelt de.pdf?6cczlv Bundeszentrale für politische Bildung [BPB]. (2016). Ein sicherer und gerechter Handlungsraum für die Menschheit. Verfügbar unter

Kapelari, Suzanne (Hg.), Vierte „Tagung der Fachdidaktik“ 2019:

„Interdisziplinäre fachdidaktische Diskurse zur Bildung für nachhaltige Entwicklung“

(C) 2020 innsbruck university press, ISBN 978-3-99106-019-2, DOI 10.15203/99106-019-2 
http://www.bpb.de/gesellschaft/umwelt/anthropozaen/248875/donut-ein-sichererund-gerechter-handlungsraum

Costanza, R., Fisher, B., Ali, S., Beer, C., Bond, L., Boumans, R., et al. (2007). Quality of life: An approach integrating opportunities, human needs, and subjective wellbeing. Ecological Economics, 61(2-3), 267-276.

doi: $10.1016 /$ j.ecolecon.2006.02.023

de Haan, G. (2010). The development of ESD-related competencies in supportive institutional frameworks. International Review of Education, 56(2-3), 315-328. doi:10.1007/s11159-010-9157-9

Dede, C. (2010). Technological supports for acquiring 21 st century skills. International encyclopedia of education, 3, 158-166.

Deutsche Gesellschaft für Geographie [DGfG] (2014). Bildungsstandards im Fach Geographie für den Mittleren Schulabschluss: mit Aufgabenbeispielen (8., aktualisierte Auflage). Bonn: Selbsterverlag DGFG. Verfügbar unter https://geographie.de/wpcontent/uploads/2014/09/geographie bildungsstandards.pdf

Emmrich, R., \& Melzer, M. (2006). Das integrative Nachhaltigkeitskonzept der HGF als Baustein der Bildung für eine nachhaltige Entwicklung. In J. Kopfmüller (Hrsg.), Ein Konzept auf dem Prüfstand: Das integrative Nachhaltigkeitskonzept in der Forschungspraxis (S. 171-188). Berlin: edition sigma.

Fischbein, E. (1987). Intuition in science and mathematics: An educational approach. Dordrecht: D. Reidel.

Fögele, J. (2016). Entwicklung basiskonzeptionellen Verständnisses in geographischen Lehrerfortbildungen. In Geographiedidaktische Forschungen: vol. 61. Münster: Verlagshaus Monsenstein \& Vannerdat OHG.

Franklin, C., Kader, G., Mewborn, D., Moreno, J., Peck, R., Perry, M., \& Scheaffer, R. (2007). Guidelines for assessment and instruction in statistics education (GAISE) report. Alexandria: American Statistical Association.

Fridrich, C., \& Hofmann-Schneller, M. (2017). Positionspapier „Sozioökonomische Bildung". GW-Unterricht, 145, 54-57. doi:10.1553/gw-unterricht145s54

Gal, I. (2002). Adults' Statistical Literacy: Meanings, Components, Responsibilities. International Statistical Review, 70(1), 1-25. doi:10.1111/j.1751-5823.2002.tb00336.x

Garfield, J. B., \& Ben-Zvi, D. (2008). Developing students' statistical reasoning: Connecting research and teaching practice. New York: Springer.

Getzin, S., \& Singer-Brodowski, M. (2016). Transformatives Lernen in einer DegrowthGesellschaft. Journal of Science-Society Interfaces, 1(1), 33-46.

Griggs, D., Stafford-Smith, M., Gaffney, O., Rockström, J., Ohman, M. C., Shyamsundar, P., et al. (2013). Policy: Sustainable development goals for people and planet. Nature, 495(7441), 305-307. doi:10.1038/495305a

Kapelari, Suzanne (Hg.), Vierte „Tagung der Fachdidaktik“ 2019:

„Interdisziplinäre fachdidaktische Diskurse zur Bildung für nachhaltige Entwicklung“

(C) 2020 innsbruck university press, ISBN 978-3-99106-019-2, DOI 10.15203/99106-019-2 
Grünwald, A., \& Kopfmüller, J. (2012). Nachhaltigkeit: Eine Einführung (2., aktualisierte Auflage). Frankfurt/New York: Campus Verlag.

Gryl, I., \& Jekel, T. (2012). Re-centring Geoinformation in Secondary Education: Toward a Spatial Citizenship Approach. Cartographica, 47(1), 18-28. doi:10.3138/carto.47.1.18

Haubrich, H., Reinfried, S., \& Schleicher, Y. (2008). Lucerne Declaration on Geographical Education for Sustainable Development. Interaction, 36(1), 39-43.

Hemmer, I. (2016). Bildung für nachhaltige Entwicklung: Der Beitrag der Fachdidaktiken. In J. Menthe, D. Höttecke, T. Zabka, M. Hammann, \& M. Rothgangel (Hrsg.), Fachdidaktische Forschungen: Vol. 10. Befähigung zu gesellschaftlicher Teilhabe: Beiträge der fachdidaktischen Forschung (S. 25-40). Münster: Waxmann.

Hodson, D. (2003). Time for action: Science education for an alternative future. International Journal of Science Education, 25(6), 645-670. doi:10.1080/09500690305021

Hoffmann, T. (2018). TERRA Globale Herausforderungen 1. Die Zukunft, die wir wollen: Themenband, Klasse 10-13. Stuttgart, Leipzig: Klett.

Holzinger, H. (2019). Dogma Wachstum. Eine kritische Würdigung der Sustainable Development Goals. Sozialwissenschaftliche Rundschau, 59(1), 6-23.

Jekel, T., \& Pichler, H. (2017). Vom GW-Unterrichten zum Unterrichten mit geographischen und ökonomischen Konzepten. $\mathrm{Zu}$ den neuen Basiskonzepten im österreichischen GW-Lehrplan AHS Sek II. GW-Unterricht, 147, 5-15. doi:10.1553/gw-unterricht147s5

Jekel, T., Gryl, I., \& Oberrauch, A. (2015). Education for Spatial Citizenship: Versuch einer Einordnung. GW-Unterricht, 137, 5-13. doi:10.1553/gw-unterricht147s5

Keil, A. (2019). Agenda 2030 und BNE: Hintergründe, aktuelle Entwicklungen und Perspektiven für das Fach Geographie. Praxis Geographie (6), 4-9.

Kennedy, J., Abichandani, P., \& Fontecchio, A. (2014). Using infographics as a tool for introductory data analytics education in 9-12. IEEE Frontiers in Education Conference (pp. 1-4). Madrid: IEEE.

Klemmer, P., Becker-Soest, D., \& Wink, R. (1998). Leitstrahlen, Leitbilder und Leitplanken: ein Orientierungsfaden für die drei grossen „L“ der Nachhaltigkeitspolitik. In A. Renner (Hrsg.), Zukunftsfähigkeit und Neoliberalismus: zur Vereinbarkeit von Umweltschutz und Wettbewerbswirtschaft (pp. 45-71). Baden-Baden: Nomos.

Knaus, A., \& Renn, O. (1998). Den Gipfel vor Augen: Unterwegs in eine nachhaltige Zukunft. Ökologie und Wirtschaftsforschung: Vol. 29. Marburg: Metropolis.

Künzli David, C., Bertschy, F., Haan, G. de, \& Plesse, M. (2008). Zukunft gestalten lernen durch Bildung für nachhaltige Entwicklung: Didaktischer Leitfaden zur

Kapelari, Suzanne (Hg.), Vierte „Tagung der Fachdidaktik“ 2019:

„Interdisziplinäre fachdidaktische Diskurse zur Bildung für nachhaltige Entwicklung“

(C) 2020 innsbruck university press, ISBN 978-3-99106-019-2, DOI 10.15203/99106-019-2 
Veränderung des Unterrichts in der Primarschule. Berlin: Transfer 21. Verfügbar unter http://www.transfer-21.de/daten/grundschule/Didaktik_Leifaden.pdf

Kultusministerkonferenz [KMK] \& Deutsche UNESCO-Kommission [DUK]. (2007). Empfehlung der Ständigen Konferenz der Kultusminister der Länder in der Bundesrepublik Deutschland (KMK) und der Deutschen UNESCO-Kommission (DUK) vom 15.06.2007 zur „Bildung für nachhaltige Entwicklung in der Schule“. Verfügbar unter

http://nachhaltigkeit.bildung-rp.de/fileadmin/user upload/nachhaltigkeit.bildungrp.de/Downloads/070615 KMK-DUK-Empfehlung BNE.pdf.

Kultusministerkonferenz $[\mathrm{KMK}] \&$ Bundesministerium für wirtschaftliche Zusammenarbeit und Entwicklung [BMZ]. (2016). Orientierungsrahmen für den Lernbereich Globale Entwicklung (2. aktualisierte und erweiterte Auflage). Bonn: Cornelsen.

Makar, K., \& Ben-Zvi, D. (2011). The Role of Context in Developing Reasoning about Informal Statistical Inference. Mathematical Thinking and Learning, 13(1-2), 1-4. doi:10.1080/10986065.2011.538291

Mehren, R., Rempfler, A., Buchholz, J., Hartig, J., \& Ulrich-Riedhammer, E. M. (2018). System competence modelling: Theoretical foundation and empirical validation of a model involving natural, social and human-environment systems. Journal of Research in Science Teaching, 55(5), 685-711. doi:10.1002/tea.21436

Ministry of Education (2007). The New Zealand curriculum. Wellington, N.Z.: Learning Media.

Niebert, K. (2016). Nachhaltigkeit lernen im Anthropozän: Wie Schule und Unterricht $\mathrm{zu}$ einer nachhaltigen Menschenzeit beitragen können. In M. K. W. Schweer (Hrsg.), Psychologie und Gesellschaft: Band 15. Bildung für nachhaltige Entwicklung in pädagogischen Handlungsfeldern: Grundlagen, Verankerung und Methodik in ausgewählten Lehr-Lern-Kontexten (S. 77-94). Frankfurt am Main: PL Academic Research.

O’Neill, D. W., Fanning, A. L., Lamb, W. F., \& Steinberger, J. K. (2018). A good life for all within planetary boundaries. Nature Sustainability, 1(2), 88-95. doi:10.1038/s41893-018-0021-4

Oberrauch, A., Keller, L., Sanin, P., \& Riede, M. (2014). Lebensqualitätsvorstellungen von Jugendlichen im Kontext des Leitbilds nachhaltiger Entwicklung. Mitteilungen der österreichischen geographischen Gesellschaft, 156, 221-248.

Ohl, U. (2013). Komplexität und Kontroversität: Herausforderungen des Geographieunterrichts mit hohem Bildungswert. Praxis Geographie (3), 4-8.

Overwien, B. (2016). Bildung für nachhaltige Entwicklung in der Schule. In M. K. W. Schweer (Hrsg.), Psychologie und Gesellschaft: Band 15. Bildung für nachhaltige Entwicklung in pädagogischen Handlungsfeldern. Grundlagen, Verankerung und

Kapelari, Suzanne (Hg.), Vierte „Tagung der Fachdidaktik“ 2019:

„Interdisziplinäre fachdidaktische Diskurse zur Bildung für nachhaltige Entwicklung“

(C) 2020 innsbruck university press, ISBN 978-3-99106-019-2, DOI 10.15203/99106-019-2 
Methodik in ausgewählten Lehr-Lern-Kontexten (S. 33-47). Frankfurt am Main: PL Academic Research.

Pfannkuch, M. (2018). Reimagining curriculum approaches. In D. Ben-Zvi, K. Makar, \& J. Garfield (Hrsg.), International Handbook of Research in Statistics Education (S. 387-413). Cham: Springer International.

Pratt, D., Davies, N., \& Connor, D. (2011). The role of technology in teaching and learning Statistics. In C. Batanero, G. Burrill, \& C. Reading (Eds.), Teaching statistics in school mathematics (S. 97-107). Dortrecht: Springer.

Prodromou, T. (2014). Drawing Inference from Data Visualisations. International Journal of Secondary Education, 2(4), 66. doi:10.11648/j.ijsedu.20140204.12

Pufé, I. (2017). Nachhaltigkeit (3., überarbeitete und erweiterte Auflage). Konstanz, München: UVK Verlagsgesellschaft $\mathrm{mbH} \& \mathrm{UVK} /$ Lucius.

Raworth, K. (2012). A Safe and Just Space for Humanity: Can we live within the doughnut? Oxfam Discussion Papers. Retrieved from https://www-cdn.oxfam.org/s3fs-public/file attachments/dp-a-safe-and-just-spacefor-humanity-130212-en 5.pdf

Raworth, K. (2018). Die Donut-Ökonomie.: Endlich ein Wirtschaftsmodell, das den Planeten nicht zerstört. München: Carl Hanser Verlag.

Reuschenbach, M., \& Schockemöhle, J. (2011). Bildung für nachhaltige Entwicklung. Leitbilder für den Geographieunterricht. Geographie heute, 295, 2-11.

Rieckmann, M. (2011). Schlüsselkompetenzen für eine nachhaltige Entwicklung der Weltgesellschaft: Ergebnisse einer europäisch-lateinamerikanischen DelphiStudie. GAIA, 48(1), 48-56.

Rieckmann, M. (2018). Learning to transform the world: key competencies in Education for Sustainable Development. In A. Leicht, J. Heiss, \& Won Jung Byun (Eds.), Issues and trends in education for sustainable development (pp. 39-59). Paris: UNESCO.

Rockström, J., Steffen, W., Noone, K., Persson, A., Chapin, F. S., Lambin, E. F., et al. (2009). A safe operating space for humanity. Nature, 461(7263), 472-475. doi:10.1038/461472a

Rosling, H., \& Rosling, O. (2014). [Video] How not to be ignorant about the world. Retrieved from https://www.ted.com/talks/hans and ola rosling how not to be ignorant about the world

Rosling, H., Rosling, O., \& Rosling-Rönnlund, A. (2018). Factfulness: Ten reasons we're wrong about the world and why things are better than you think. London: Sceptre.

Rychen, S., \& Salganik, L. H. (Eds.). (2003). Key competencies for a successful life and well-functioning society. Cambridge: Hogrefe und Huber.

Kapelari, Suzanne (Hg.), Vierte „Tagung der Fachdidaktik“ 2019:

„Interdisziplinäre fachdidaktische Diskurse zur Bildung für nachhaltige Entwicklung“

(C) 2020 innsbruck university press, ISBN 978-3-99106-019-2, DOI 10.15203/99106-019-2 
Schreiner, C., Henriksen, E. K., \& Kirkeby Hansen, Pål J. (2005). Climate Education: Empowering Today's Youth to Meet Tomorrow's Challenges. Studies in Science Education, 41(1), 3-49. doi:10.1080/03057260508560213

Skranefjell, A., \& Tønnessen, M. (2003). Statistical storytelling. Statistical Journal of the United Nations Economic Commission for Europe, 20(1), 51-54.

Steffen, W., Richardson, K., Rockstrom, J., Cornell, S. E., Fetzer, I., Bennett, E. M., et al. (2015). Sustainability. Planetary boundaries: guiding human development on a changing planet. Science, 347(6223), 736-746. doi:10.1126/science.1259855

Stoltenberg, U., \& Fischer, D. (2018). Bildung für eine nachhaltige Entwicklung in Deutschland: vom Projekt zur Struktur? In Forum Umweltbildung (Hrsg.), Perspektive wechseln. Jahrbuch Bildung für nachhaltige Entwicklung (S. 76-89). Wien.

Taspolat, A., Hamza, K., Sami, O., Fezile, B., \& Mobina, F. (2017). An investigation toward advantages, design principles and steps of infographics in education. International Journal of Science and Research, 73(7), 157-166.

Taylor, N., Quinn, F., Jenkins, K., Miller-Brown, H., Rizk, N., Prodromou, T., ... Taylor, S. (2019). Education for Sustainability in the Secondary Sector-A Review. Journal of Education for Sustainable Development, 13(1), 102-122.

Tversky, A., \& Kahneman, D. (1974). Judgment under uncertainty: Heuristics and biases. Science, 185, 1124-1131.

United Nations [UN] (2015). Sustainable Development Goals. Retrieved from https://sustainabledevelopment.un.org/?menu=1300

Vare, P., \& Scott, W. (2007). Learning for a Change: Exploring the Relationship between Education and Sustainable Development. Journal of Education for Sustainable Development, 1(2), 191-198. doi:10.1177/097340820700100209

von Hauff, M. \& Kleine, A. (2009). Nachhaltige Entwicklung. Grundlagen und Umsetzung. München: Oldenbourg Verlag.

Vorage, M. (2019). Nachhaltige Bildung für nachhaltige Entwicklung. GW-Unterricht, 154, 48-56. doi:10.1553/gw-unterricht154s48

Wassong, T., Frischemeier, D., Fischer, P. R., Hochmuth, R., \& Bender, P. (Hrsg.). (2014). Mit Werkzeugen Mathematik und Stochastik lernen - Using Tools for Learning Mathematics and Statistics. Wiesbaden: Springer Spektrum. doi:10.1007/978-3-658-03104-6

Wiek, A., Withycombe, L., \& Redman, C. L. (2011). Key competencies in sustainability: A reference framework for academic program development. Sustainability Science, 6(2), 203-218. doi:10.1007/s11625-011-0132-6

Wild, C. J., \& Pfannkuch, M. (1999). Statistical Thinking in Empirical Enquiry. International Statistical Review, 67(3), 223-248.

doi:10.1111/j.1751-5823.1999.tb00442.x

Kapelari, Suzanne (Hg.), Vierte „Tagung der Fachdidaktik“ 2019:

„Interdisziplinäre fachdidaktische Diskurse zur Bildung für nachhaltige Entwicklung“

(C) 2020 innsbruck university press, ISBN 978-3-99106-019-2, DOI 10.15203/99106-019-2 


\title{
Vom Auswahlmodus zur Bewertungskompetenz
}

\author{
Mag. ${ }^{a}$ Christine Wogowitsch, Universität Innsbruck
}

Mit der im 19. Jahrhundert beginnenden Industrialisierung überstiegen die Auswirkungen anthropogener Einflüsse die Selbstregulierungskräfte der Erde und führten $\mathrm{zu}$ globalen Umweltauswirkungen, die zunehmend Tier- und Pflanzenarten und somit Lebensräume für Menschen und alle anderen Lebewesen gefährden. Mit der Möglichkeit in Abläufe einzugreifen wird zunehmend bewusst wahrgenommen, dass langfristige Auswirkungen auf das Klima unkalkulierbar werden und einer globalen Anstrengung bedürfen (Niebert, 2016). Bereits im Jahr 2002 wurde bei der Konferenz für Umwelt und Entwicklung in Johannesburg festgestellt, dass der Bildung ein viel höheres Gewicht beizumessen ist, um die gesamte Menschheit zur Mitverantwortung zu befähigen (Steiner \& Rauch, 2013).

Vorliegender Beitrag geht der Frage nach, wie Lernende zur Verantwortungsübernahme und zur Entwicklung von Werthaltungen im Sinne einer emanzipatorischen BNE angeleitet werden können, um zu Fragen der Solidarität und Gerechtigkeit Stellung zu beziehen und so zu GestalterInnen einer zukunftsfähigen Entwicklung werden. 


\section{Bildungspolitischer Rahmen}

Österreich bekennt sich mit dem Bundesverfassungsgesetz vom 11. Juli 2013 (BGB1. I Nr. 111/2013) im $\S 1$ zum Prinzip der Nachhaltigkeit bei der Nutzung der natürlichen Ressourcen, um auch zukünftigen Generationen bestmögliche Lebensqualität zu gewährleisten (Republik Österreich, 2013). Mit der Österreichischen Strategie zur Bildung für nachhaltige Entwicklung soll der Bewusstseinswandel in Richtung Nachhaltigkeit bei Lernenden und Lehrenden unterstützt und die AkteurInnen vernetzt werden (Iwaniewicz, Pfaffenwimmer, $\&$ Frei, 2008).

Die Zusammenfassung und der Endbericht des UNECE-Reports 2014 hebt in der Beschreibung von Handlungsfeldern die Rolle der Bildung für eine nachhaltige Entwicklung (BNE) durch die Sicherstellung geeigneter Rahmenbedingungen hervor (Frei, Iwaniewicz, Pfaffenwimmer, Steiner \& Streissler, 2014).

Ausgehend von theoretischen Konstrukten, Projektberichten und gesetzlichen Grundlagen werden von der Autorin Überlegungen für die Integration einer nachhaltigen Bildung im Unterricht an berufsbildenden Schulen angestellt.

\subsection{Gesetzliche Grundlage}

Werden gesetzliche Vorgaben aus fachübergreifender Perspektive betrachtet, so bilden Unterrichtsprinzipien den zentralen Anhaltspunkt. Von den insgesamt 14 Unterrichtsprinzipien, die im Österreichischen Bildungssystem für alle Schulstufen, Schultypen und Schulformen gelten und fächerübergreifend in jedem Unterrichtsgegenstand vermittelt werden sollen, nehmen die beiden Unterrichtsprinzipien ,Politische Bildung' (Grundsatzerlass 2015, BMBF33.466/0029-I/6/2015) und ,Umweltbildung für nachhaltige Entwicklung' (Grundsatzerlass 2014, BMBF-37.888/0062-I/6c/2014) (Bundesministerium für Bildung und Frauen, 2014) eine besondere Stellung ein. SchülerInnen sollen entsprechend dieser beiden Grundsatzerlässe Kompetenzen erwerben, um sich aktiv und kritisch an umwelt- und gesellschaftlichen Herausforderungen im

Kapelari, Suzanne (Hg.), Vierte „Tagung der Fachdidaktik“ 2019:

„Interdisziplinäre fachdidaktische Diskurse zur Bildung für nachhaltige Entwicklung“

(C) 2020 innsbruck university press, ISBN 978-3-99106-019-2, DOI 10.15203/99106-019-2 
Privat- und Berufsleben im Spannungsfeld unterschiedlicher Wertvorstellungen, Bedürfnisse und Interessen beteiligen $\mathrm{zu}$ können. Die inhaltlichen Rahmenvorgaben der Unterrichtsprinzipien ,Politische Bildung' und ,Umweltbildung für nachhaltige Entwicklung' sind in Grundsatzerlässen festgehalten. Der Grundsatzerlass ,Umweltbildung für nachhaltige Entwicklung' präzisiert die schulischen Herausforderungen durch konkrete Ziele, Kompetenzen, Prinzipien, thematische Zugänge und durch methodischdidaktische Grundsätze.

\subsection{Bildung für nachhaltige Entwicklung (BNE)}

Vor dem Hintergrund der weltweiten ökologischen, sozialen, ökonomischen und kulturellen Probleme wurde von der UN-Konferenz für Umwelt und Entwicklung 1992 in Rio de Janeiro das Leitbild einer nachhaltigen Entwicklung skizziert. Dynamische Veränderungen erfordern einen gesellschaftlichen Lern- und Verständigungsprozess, der über traditionelle Wissensbestände und konkrete Verhaltensempfehlungen hinausgeht. Bildung soll Bewusstsein für nachhaltigkeitsrelevante Probleme schaffen und den Erwerb von Wissen über diese Probleme ermöglichen (Rieckmann, 2016, S. 90). Angesichts unklarer Problemlagen müssen AkteurInnen imstande sein, unter sich verändernden Bedingungen flexibel zu reagieren, Entscheidungen abzuwägen und handlungsfähig zu bleiben. Demnach zielt BNE darauf ab, den Erwerb sogenannter Schlüsselkompetenzen $\mathrm{zu}$ fördern, um ein bewusstes Handeln im Sinne einer nachhalten Entwicklung zu unterstützen (Rieckmann, Fischer, \& Richter, 2014).

\subsubsection{Strömungen in der BNE}

BNE kommt demnach eine Schlüsselrolle $\mathrm{zu}$, indem sie ein fundiertes Verständnis der Herausforderungen und einen kritischen Diskurs über mögliche Lösungswege sowie die Entwicklung eines Bewusstseins für nachhaltige Handlungsalternativen forciert. In der BNE werden zwei Strömungen

Kapelari, Suzanne (Hg.), Vierte „Tagung der Fachdidaktik“ 2019:

„Interdisziplinäre fachdidaktische Diskurse zur Bildung für nachhaltige Entwicklung“

(C) 2020 innsbruck university press, ISBN 978-3-99106-019-2, DOI 10.15203/99106-019-2 
unterschieden. BNE 1, „Instrumentelle BNE“ und BNE 2 „Emanzipatorische BNE“. Eine instrumentelle BNE verlangt als normatives Konzept von Bildung jene gesellschaftlichen Transformationsprozesse, Verhaltensweisen und Lebensstile zu erlernen, die für eine positive Zukunft und für eine positive gesellschaftliche Transformation notwendig sind. Zukunftsverantwortung bedeutet in diesem Zusammenhang, dass die Vorsorge-, Verursacher- und Haftungsprinzipien als grundlegende Rahmenbedingungen für ein langfristig tragfähiges wirtschaftliches, ökologisches und gesellschaftliches Handeln und Verhalten auf allen Ebenen zu fördern sind. Eine emanzipatorische BNE geht von der Befähigung von Individuen aus, in kontroversen Diskursen zu partizipieren und gesellschaftliche Leitbilder, Normen und Werte einer kritischen Reflexion zu unterziehen um autonom handeln zu können. Die individuellen umweltrelevanten Einstellungen und Werte sind dabei einer laufenden Analyse zu unterziehen, damit die Handlungskonsequenzen sichtbar werden. Nachhaltige Entwicklung wird damit als regulative Idee verstanden, welche als dauerhafte Aufgabe den gesellschaftlichen Lern- und Gestaltungsprozess inspirieren soll (Rieckmann, 2016, S. 91) und (KyburzGraber, et al., 2006).

\subsubsection{BNE und Werte}

Standop charakterisiert den Begriff ,Wert' als eine grundlegende Vorstellung über erwünschte Endzustände, der für das Bestreben eines Individuums oder einer Gruppe charakteristisch ist und einer kulturellen Prägung unterliegt. Werte zeigen sich im schulischen Kontext durch verschiedene Verhaltensweisen oder Meinungsäußerungen, geben Aufschluss über Ziele oder Motive und entwickeln sich durch Erfahrung (2016, S. 13-17). Die individuellen Werthaltungen nehmen Einfluss auf Motivation und somit über Intensionsbildung auf das Verhalten (Payrhuber, 2013, S. 25). In Bildungsprozessen für eine nachhaltige Entwicklung spielen Werthaltungen sowohl auf der Handlungsebene durch eine ,Instrumentelle BNE' realisiert, als auch auf der reflektierenden Ebene als soziale Bedingungen des Erlebens und

Kapelari, Suzanne (Hg.), Vierte „Tagung der Fachdidaktik“ 2019:

„Interdisziplinäre fachdidaktische Diskurse zur Bildung für nachhaltige Entwicklung“

(C) 2020 innsbruck university press, ISBN 978-3-99106-019-2, DOI 10.15203/99106-019-2 
Handelns umgesetzt durch eine ,Emanzipatorische BNE' eine wichtige Rolle. Über die Auseinandersetzung mit Fragen der Verantwortung für den Erhalt natürlicher Lebensgrundlagen, der Menschenwürde, Offenheit, Solidarität, Gerechtigkeit, Ehrlichkeit, Toleranz oder Respekt können die eigenen Werte reflektiert, geklärt und einem kritischen Diskurs unterzogen werden, um den Wertehorizont der Lernenden $\mathrm{zu}$ erweitern (Frey, 2016). Fragen zu Ressourcenverbrauch, -qualität, und -verteilung verlangen Weitsichtigkeit, Achtung vor der Natur und nach einem Handeln im Bewusstsein globaler Verantwortung.

\subsection{Agenda 2030}

Die UN-Generalversammlung verabschiedete am 25. September 2015 eine Resolution zur Transformation unserer Welt durch die Agenda 2030 für nachhaltige Entwicklung. Die Agenda verfolgt die Absicht, durch einen globalen Aktionsplan, mit den 17 Zielen für eine nachhaltige Entwicklung und den 169 Zielvorgaben einer nachhaltigen Entwicklung auf ausgewogener Weise der wirtschaftlichen, ökologischen und sozialen Dimension Rechnung zu tragen. (Vereinte Nationen, 2015).

Das übergreifende Ziel der Agenda 2030 ist, Aktivitäten auf allen Ebenen und in allen Bereichen anzustoßen und zu intensivieren, um den Prozess hin zu einer nachhaltigen Entwicklung zu beschleunigen. Die Gestaltung der Zukunft im Sinne der Agenda 2030 wird von den wertebasierten Entscheidungen der Menschen geprägt. Eine Sensibilisierung für wertebezogene Ziele und die Verantwortungsübernahme sind deshalb als zentrale Aufgaben schulischer Bildungsarbeit zu verstehen. Dabei werden zwei zentrale Ziele genannt, die in schulischen Bildungsaktivitäten $\mathrm{zu}$ verfolgen sind: (1) „Neuorientierung von Bildung und Lernen, sodass jeder die Möglichkeit hat, sich das Wissen, die Fähigkeiten, Werte und Einstellungen anzueignen, die erforderlich sind, um zu einer nachhaltigen Entwicklung beizutragen. (2) „Stärkung der Rolle von Bildung und Lernen in allen Projekten, Programmen und Aktivitäten, die sich für eine nachhaltige Entwicklung einsetzen“" (Deutsche UNESCO-Kommission,

Kapelari, Suzanne (Hg.), Vierte „Tagung der Fachdidaktik“ 2019:

„Interdisziplinäre fachdidaktische Diskurse zur Bildung für nachhaltige Entwicklung“

(C) 2020 innsbruck university press, ISBN 978-3-99106-019-2, DOI 10.15203/99106-019-2 
2014, S. 14). Das in der Agenda 2030 beschriebene Ziel 12 „für nachhaltige Konsum- und Produktionsmuster sorgen", konkretisiert die Anliegen einer tragfähigen sozialen und wirtschaftlichen Entwicklung, wobei der Reduktion von Lebensmittelabfall eine entscheidende Rolle zukommt (United Nations, 2019). Zur Realisierung dieses Ziels bietet Ernährung ein zentrales Handlungsfeld für eine nachhaltige Entwicklung und kann weitreichende interdisziplinäre Bezüge eröffnen. So stellen die Bekämpfung des Welthungers, eine umweltverträgliche, soziale und faire Gestaltung der Landwirtschaft, eine Verringerung der Menge freigesetzter klimarelevanter Gase, eine Reduzierung der Lebensmittelverschwendung oder die Verbesserung der menschlichen Gesundheit sowohl berufliche Tätigkeitsbereiche als auch Kommunikationsund Reflexionsräume im Sinne von BNE 1 und 2 dar und sind Anliegen, die eng mit der Frage verknüpft sind, wie wir unsere Lebensmittel produzieren und konsumieren (Rieckmann, Fischer, \& Richter, 2014).

Im Dissertationsprojekt wurden die curricularen Ansprüche mit dem Ziel 12 ,Nachhaltige/r Konsum und Produktion" (Ökobüro-Allianz der Umweltbewegung, 2017) zur Entwicklung von Werthaltungen hinsichtlich verantwortungsvoller Konsum- und Produktionsmuster herangezogen.

\subsection{Konzepte einer nachhaltigen Entwicklung}

Johan Rockström vom Stockholm Resilience Centre und internationale WissenschaftlerInnen formulierten in dem 2009 publizierten Fachartikel „A safe operating space for humanity“ planetare Belastbarkeitsgrenzen, welche die ökologischer Belastbarkeitsgrenzen der Erde einerseits auf naturwissenschaftlichen Erkenntnissen, andererseits auf der Anwendung des Vorsorgeprinzips beschreiben. Die vier Kernaussagen des Konzepts beziehen sich auf den Rückgang der biologischen Vielfalt, die Klimaveränderungen und die Reduzierung der Waldflächen, die Nutzung von Süßwasser, den Ozonverlust in der Stratosphäre und die Versauerung der Meere und den Aerosolgehalt der Atmosphäre (Rockstroem, Steffen, Richardson, Cornell, \& Fetzer, 2015). Dieses Theoriemodell bietet einen zentralen Anhaltspunkt für die

Kapelari, Suzanne (Hg.), Vierte „Tagung der Fachdidaktik“ 2019:

„Interdisziplinäre fachdidaktische Diskurse zur Bildung für nachhaltige Entwicklung“

(C) 2020 innsbruck university press, ISBN 978-3-99106-019-2, DOI 10.15203/99106-019-2 
didaktische Erarbeitung von Nachhaltigkeitsthemen und kann mit aktuellen Bezügen zur thematischen Fokussierung beitragen. Berechnungsmodelle wie der ,Happy Planet Index', der ,Human Development Index', der ,ökologische Fußabdruck' oder der ,ökologische Rucksack' berechnen soziale, ökonomische und ökologische Aspekte und zeigen die Auswirkungen menschlichen Handelns. Sie unterscheiden sich jedoch in der verwendeten Methodologie, nach Datenlage und regionaler Prioritätensetzung (Industrie- und Handelskammer Nürnberg für Mittelfranken, 2015). Werden diese Modelle im Unterricht in unterschiedlichen thematischen Kontexten verwendet, so können die von den Lernenden ermittelten Ergebnisse zu mehrperspektivischer Betrachtung und zu Diskussionen anleiten, ihr bisheriges Handeln hinsichtlich der Nachhaltigkeitsziele infrage stellen und Irritationen auslösen.

Die angeführten Dokumente und Quellen bilden den Rahmen für die Beantwortung der Frage, wie im Dissertationsprojekt Nachhaltigkeit als fruchtbares Element im berufsbildenden Unterricht durch kognitive, emotionale und handlungsorientierte Entwicklung zu einem ethisch-verantwortungsvollen Handeln im persönlichen Alltag und im beruflichen Handeln zu einer Stabilisierung des Klimawandels beitragen kann (siehe dazu 4.5: Erste Forschungsergebnisse).

\section{Der curriculare Rahmen für eine Berufsbildung für nachhaltige Entwicklung}

\subsection{Berufsbildung}

Zum System der beruflichen Bildung in Österreich zählen die schulischbetrieblich duale Ausbildung in Form der Lehre, die überbetriebliche Ausbildung sowie die berufsbildenden mittleren und höheren Schulen (Schlögl, Stock, \& Mayerl, 2019). Nach der Internationalen Standardklassifikation im Bildungswesen (ISCED) führen diese zu den Ausbildungsniveaus drei bis fünf (Österreichischer Austauschdienst, 2014). Nach Fischer und Raschpichler

Kapelari, Suzanne (Hg.), Vierte „Tagung der Fachdidaktik“ 2019:

„Interdisziplinäre fachdidaktische Diskurse zur Bildung für nachhaltige Entwicklung“

(C) 2020 innsbruck university press, ISBN 978-3-99106-019-2, DOI 10.15203/99106-019-2 
(2012) verfolgt die Berufsbildung eine doppelte Zielsetzung: einerseits sollen Lernende die Berufsfähigkeit erlangen, welche Fachkompetenz mit sozialen Fähigkeiten verbindet und andererseits soll auf die beruflichen Anforderungen mit Flexibilität reagiert werden. Bei der insbesondere in der Berufsbildung etablierten Gegenstandszentrierung stand das jeweilige Unterrichtsfach lange Zeit alleinig im Zentrum der Bildungsarbeit. Damit einhergehend war es das vorrangige Ziel der Lehrenden, einen inhaltlichen Gegenstandsbereich systematisch aufzubereiten, ihn didaktisch zu rekonstruieren, um diesen dann über den Weg der direkten Instruktion an die Lernenden weiterzugeben (Fritz \& Willenshofer, 2012).

Nachhaltige Bildung in berufsbildenden Schulen wurde je nach Ausbildungszweig und den Interessen der Lehrpersonen an Nachhaltigkeitsthemen sehr unterschiedlich interpretiert und in das Unterrichtsgeschehen integriert. Die OECD Untersuchung zur Berufsbildung in Österreich soll Ländern helfen, ihre Berufsbildungssysteme stärker auf die Arbeitsmarkterfordernisse abzustimmen (OECD, Bildung, Abteilung Bildungsund Ausbildungspolitik, 2019). Eine Recherche dieses Berichts über die Nennung des BNE-Begriffes ergab weder in den dokumentierten Stärken und Herausforderungen noch in den Empfehlungen den gesuchten Hinweis. Lediglich auf die Anwendung zeitgemäßer Methoden wird hingewiesen, woraus Rückschlüsse auf eine nachhaltige Bildung abgeleitet werden könnten (Hoeckel, 2010).

Mit der ab dem Jahr 2012 beginnenden Einführung des kompetenzorientierten Unterrichts wird Nachhaltige Bildung erstmals flächendeckend in die Bildungsstandards aufgenommen und zum integralen Bestandteil der beruflichen Bildung (Bundesministerium für Bildung und Frauen, 2015).

Die Lehrpläne der mittleren und höheren Schulen für wirtschaftliche Berufe oder für Landwirtschaft zeigen umfangreiche Schwerpunktsetzungen für eine Berufsbildung für nachhaltige Entwicklung (Fritz \& Willenshofer, 2012). Exemplarisch sei hier auf die Bildungsstandards der höheren land- und forstwirtschaftlichen Schulen (HLFS)-Forstwirtschaft hingewiesen (Veis, 2017).

Kapelari, Suzanne (Hg.), Vierte „Tagung der Fachdidaktik“ 2019:

„Interdisziplinäre fachdidaktische Diskurse zur Bildung für nachhaltige Entwicklung“

(C) 2020 innsbruck university press, ISBN 978-3-99106-019-2, DOI 10.15203/99106-019-2 
Für die Berufsbildung stellt die Integration nachhaltiger Sichtweisen eine besonders große Herausforderung dar und bietet vor allem in der dualen Ausbildung ein ideales Lernfeld für Aushandlungsprozesse, wo betriebliche Anforderungen auf Umweltanliegen und soziale Anliegen treffen. Eine steigende Anzahl von Unternehmen erkennt die Chancen, die sich aus der nachhaltigen Entwicklung auf ökonomischer, ökologischer und sozialer Basis ergeben (Rieckmann, 2016, S. 26).

\subsection{Fachbereich Naturwissenschaften}

Für das Dissertationsprojekt wurde der kompetenzorientierte Lehrplan der dreijährigen Fachschule für wirtschaftliche Berufe (BGBl. II Nr. 340 vom 17. November 2015, Anlage A3) (Bundesministerium für Bildung, 2015) systematisch nach Andockstellen für BNE analysiert. Diese Untersuchung ergab im Fachbereich ,Naturwissenschaften' in den Modulen drei bis sechs sieben zentrale Themenbereiche, welche in den Dimensionen Ökologie, Ökonomie und Soziales zur Entwicklung der UNESCO Nachhaltigkeitskompetenzen (UNESCO, 2017) einen wesentlichen Beitrag leisten können. In der Abb. 2 wird die Interdependenz von Ökologie, Ökonomie und Gesellschaft mit dem Ergebnis der Curriculaanalyse durch die angeführten Themenbereiche dargestellt. Eine nachhaltigkeitsorientierte Berufsbildung kann durch Lernanlässe mit Berufs- und Lebensweltbezug in gemeinschaftlicher Erarbeitung und Reflexion auf individueller, lokaler, regionaler, nationaler und globaler Ebene zur Lösung gegenwärtig anstehender Probleme und zum Entwurf visionärerer Zukunftsbilder beitragen.

Kapelari, Suzanne (Hg.), Vierte ,Tagung der Fachdidaktik“ 2019:

„Interdisziplinäre fachdidaktische Diskurse zur Bildung für nachhaltige Entwicklung“

(C) 2020 innsbruck university press, ISBN 978-3-99106-019-2, DOI 10.15203/99106-019-2 


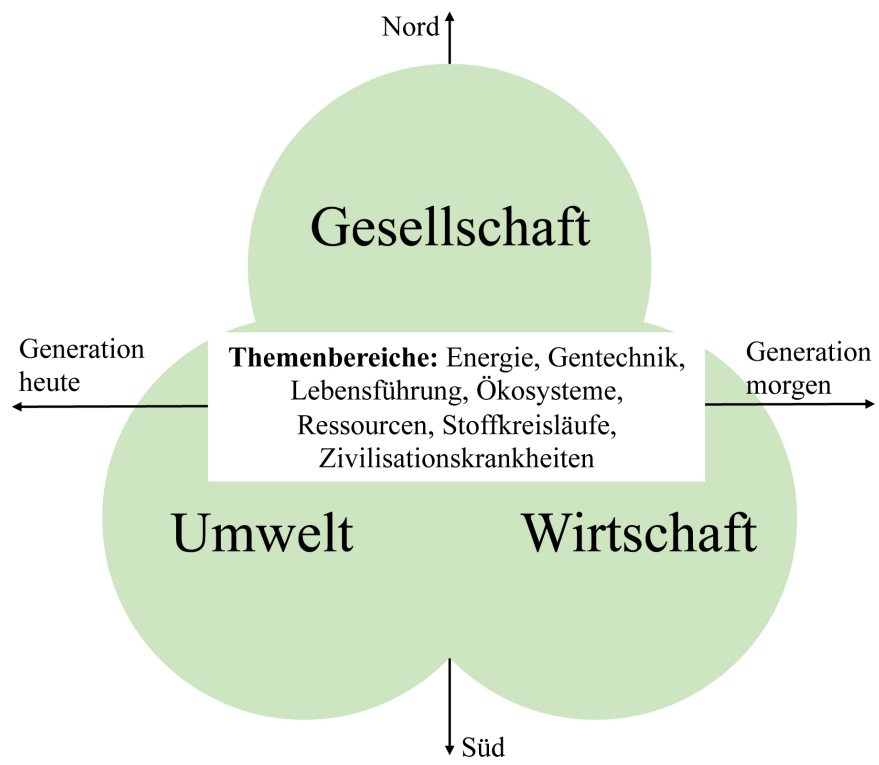

Abb. 1: Dimensionen nachhaltiger Entwicklung und Themenbereiche; eigene Darstellung nach (Kocher, 2016)

Weitere Unterrichtsgegenstände, wie z.B. „Ernährungslehre', der ,fachpraktische Unterricht' ,Küchen- und Restaurantmanagement' und ,Betriebsorganisation mit Übungen' ermöglichen aufgrund im Lehrplan angeführter fachübergreifender Themenbereiche den Transfer in interdisziplinäre Settings. Sie bieten Anknüpfungspunkte zur Bewältigung und Lösung von Problemen mit Lebensweltbezug, eine empathische, tiefgründige und mehrperspektivische Auseinandersetzung und erzeugen eine emotionale Betroffenheit, wie z.B. bei einem Lernprozess über den individuellen und globalen Wasserverbrauch in Verbindung mit der Wassernutzung aus individueller und globaler Perspektive. Einen weiteren thematischen Zugang für eine mehrperspektivische Betrachtung im Sinne der BNE ermöglicht der Themenbereich ,Nachhaltiges Ernährungssystem'. 


\subsection{Fachbereich Naturwissenschaften}

Das Potenzial des Themenbereichs Ernährung mit seiner globalen und zukunftsweisenden Ausrichtung reicht weit über die konkrete Unterrichtsarbeit hinaus und übt durch das Speisenangebot in Mensen und bei Schulbuffets Einfluss auf das Nachhaltigkeitsverständnis an einer Schule sowie auf das individuelle Ernährungsverhalten und -bewusstsein der Lernenden aus (Rieckmann, Fischer, \& Richter, 2014).

Im Unterricht sind gemäß den gesetzlichen Grundlagen (BGBl. II Nr. 340 vom 17. November 2015, Anlage A3) (Bundesministerium für Bildung, 2015) Einsichten in ökonomische, ökologische und soziale Zusammenhänge im Kontext globaler und lokaler Bezüge zu erarbeiten und gerechte, ethisch vertretbare zukunftsorientierte Handlungsoptionen $\mathrm{zu}$ entwickeln. Der enge Zusammenhang zwischen Energie-, Finanz- und Klimafragen, der Erhalt bzw. die Schonung natürlicher Ressourcen und der Wert einer biologischen Vielfalt bilden relevante Anknüpfungspunkte und stellen den Berufsfeldbezug her. In diesem Kontext ermöglichen Ernährungssysteme bei der eigenverantwortlichen Auswahl, Verarbeitung und Qualitätsbeurteilung von Lebensmitteln oder bei anderen Konsumgütern sowohl im beruflichen als auch im privaten Bereich anspruchsvolle Lernanlässe mit hohem Reflexionspotenzial. 


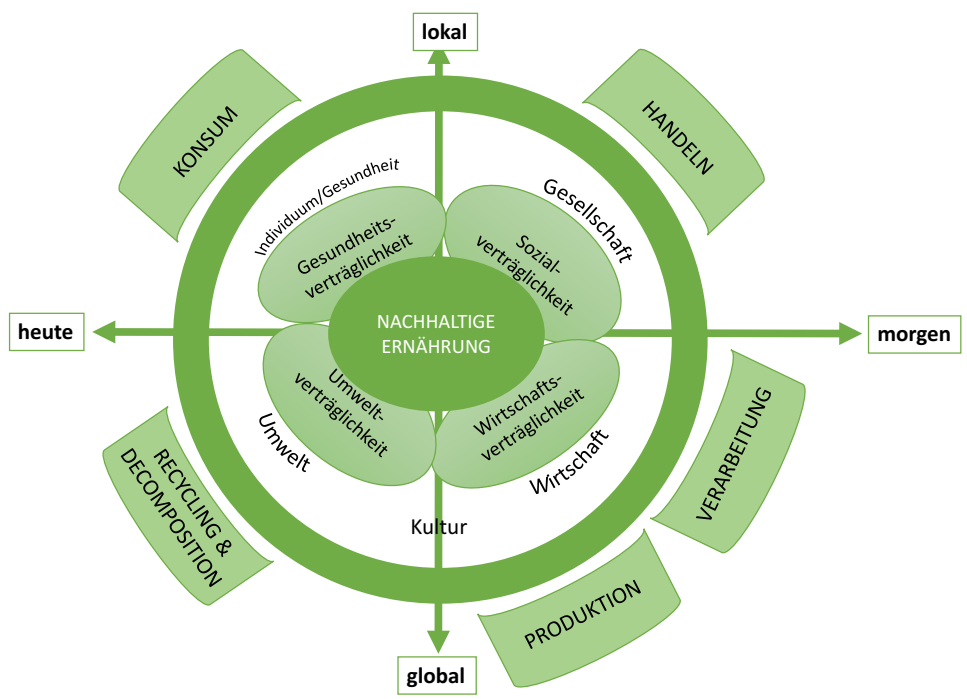

Abb. 2: Modell eines nachhaltigen Ernährungssystems (eigene Darstellung)

In diesem Zusammenhang soll bei Lernenden ein Bewusstsein für die Verflechtung sozialer, kultureller, ökologischer und ökonomischer Aspekte entstehen. Über Erfahrungen aus dem theoretischen und fachpraktischen Unterricht sowie aus der Berufspraxis können Lernende konstruktiv und handlungsorientiert an Themen anknüpfen und den Wissenserwerb mit emotionalen Erlebnissen stützen. Beispielhaft sei hier Produktion, Verarbeitung, Verteilung, Konsum und Entsorgung von Lebensmitteln genannt, wodurch ein forschender, konstruktiv angelegter Prozess entstehen kann. Dieser bietet sich an, persönliche Denk- und Wertekonzepte zu überprüfen, wie z.B. durch ,Reflexion über Themen aus Gesellschaft, Politik und Wirtschaft' im Fach Deutsch und im Fach Englisch durch, mündliche und schriftliche Kommunikation' zu ,Tourismus, Ernährung, Gesundheit, zu Lebenswirklichkeiten Jugendlicher in verschiedenen Ländern, Werbung und Kultur' oder im Fach Biologie über den Lehrstoff ,Konsum und Nachhaltigkeit' oder ,Ursachen und Folgen des Klimawandels' (BGB1. II Nr. 340 vom 17. November 2015, Anlage A3) (Bundesministerium für Bildung, 2015, S. 17, 22, 27). 


\section{Didaktik einer werte- und nachhaltigkeitsorientierten Berufsbildung}

Die Integration von BNE in das berufsbildende Schulwesen erfordert didaktische Strategien (Forstner-Ebhart \& Linder, 2019, S. 10-21). Nach Robinsohn (1971) hat die Schule die Aufgabe zur Bewältigung von Lebenssituationen des Einzelnen beizutragen. Diese Logik findet in Anlehnung an die Ansprüche einer nachhaltigen Bildung ihre Fortsetzung bei Kai Niebert (2016, S. 88-91): „es reicht aus fachdidaktischer Sicht nicht, allgemeine Grundsätze des Erkennens, Bewertens, Reflektierens, der Partizipation und des Handelns in heterogenen Gruppen usw. zu formulieren, die das Individuum befähigen sollen, aktiv, reflektiert und eigenverantwortlich entscheiden und handeln zu können. All diese Entscheidungen, Reflexionen und Handlungen finden immer in konkreten Kontexten statt." Aus diesem Verständnis heraus befähigt BNE bei der Auseinandersetzung mit vorgegebenen Zielen zu einem kritischen Wertediskurs. Die Grundlage für Überlegungen zur Beschreibung eines pädagogischen Ansatzes für eine werte- und nachhaltigkeitsorientierte Berufsbildung bilden die Reflexion fachlicher Basiskonzepte und die MenschUmwelt-Beziehungen von Lernenden zur Erreichung spezifischer kognitiver, sozioemotionaler und verhaltensbezogener Lernergebnisse. Sie sollen dadurch die Fähigkeit erlangen, mit den beruflichen und privaten Herausforderungen so umzugehen, dass sie den gesellschaftlichen, wirtschaftlichen und politischen Wandel im Sinne einer nachhaltigen Entwicklung fördern und ihr eigenes Verhalten dementsprechend ausrichten (Stiftung Zukunftsrat und Baustelle Zukunft, 2018). Dieser Argumentation folgend, gilt es zu erörtern, welche Entscheidungsgrundlage $\mathrm{zu}$ schaffen ist, damit Bestimmungen für die didaktische Gestaltung von Lernsettings in der Berufsbildung formuliert werden können. Im Dissertationsprojekt wurden für die Gestaltung von Lernanlässen im Einklang mit der von de Haan (2002, S. 13-20) beschriebenen Gestaltungskompetenz und den UNESCO-Nachhaltigkeitskompetenzen (OECD, Bildung, Abteilung Bildungs- und Ausbildungspolitik, 2019) mit den beiden Ansätzen der instrumentellen und emanzipatorischen BNE (siehe dazu Abs. 1.2) verknüpft.

Kapelari, Suzanne (Hg.), Vierte „Tagung der Fachdidaktik“ 2019:

„Interdisziplinäre fachdidaktische Diskurse zur Bildung für nachhaltige Entwicklung“

(C) 2020 innsbruck university press, ISBN 978-3-99106-019-2, DOI 10.15203/99106-019-2 
Um den Kompetenzerwerb in der Berufsbildung wirkungsvoll zu gestalten, ist eine partizipative über Fachgrenzen hinausgehende Zusammenarbeit erforderlich, da z.B. der Problematik des globalen Klimawandels nur in interdisziplinärer Gemeinsamkeit begegnet werden kann. Die Flexibilität organisationaler Strukturen erhöht dabei das Potenzial für kollektives Lernen (Wogowitsch, 2016).

Tab. 1: Schlüsselkompetenzen und didaktische Prinzipien einer werte- und nachhaltigkeitsorientierten Bildung; eigene Darstellung nach Rieckmann (2019).

\begin{tabular}{|l|l|}
\hline $\begin{array}{l}\text { UNESCO- } \\
\text { Nachhaltigkeits- } \\
\text { kompetenzen }\end{array}$ & $\begin{array}{l}\text { Didaktische Prinzipien für eine BNE-spezifische } \\
\text { Handlungsstruktur }\end{array}$ \\
\hline Vernetztes Denken & $\begin{array}{l}\text { Vielfältige Phänomene und gesellschaftliche } \\
\text { Herausforderungen aus der Lebens- und Erfahrungswelt } \\
\text { der Lernenden als Lernanlässe aufbereiten um } \\
\text { Zusammenhänge zu erkennen, Verständnis zu entwickeln, } \\
\text { den Umgang mit Unsicherheiten einzuüben und Visionen } \\
\text { zu entwickeln. }\end{array}$ \\
\hline $\begin{array}{l}\text { Vorausschauendes } \\
\text { Denken }\end{array}$ & $\begin{array}{l}\text { Vultiple Zukunftsentwürfe schaffen und dabei das } \\
\text { Handlungen beurteilen, Risiken und Veränderungen } \\
\text { konstruktiv begegnen um Handlungskompetenz zu } \\
\text { erwerben. }\end{array}$ \\
\hline Normative Kompetenz & $\begin{array}{l}\text { Normen und Werte verstehen und reflektieren, dabei } \\
\text { Interessenkonflikten und Widersprüchen in } \\
\text { Dilemmasituationen durch empathisch geführte } \\
\text { Aushandlungsprozesse begegnen. }\end{array}$ \\
\hline Strategische Kompetenz & $\begin{array}{l}\text { Innovative Maßnahmen kollektiv entwickeln und durch } \\
\text { Beteiligung an Nachhaltigkeitsprozessen auf lokaler } \\
\text { Ebene umsetzen. }\end{array}$ \\
\hline Kooperationskompetenz & $\begin{array}{l}\text { Von und mit anderen in kollaborativen und partizipativen } \\
\text { Prozessen Partizipationsorientierung lernen und Probleme } \\
\text { lösen. }\end{array}$ \\
\hline
\end{tabular}

Kapelari, Suzanne (Hg.), Vierte „Tagung der Fachdidaktik“ 2019:

„Interdisziplinäre fachdidaktische Diskurse zur Bildung für nachhaltige Entwicklung“

(C) 2020 innsbruck university press, ISBN 978-3-99106-019-2, DOI 10.15203/99106-019-2 


\begin{tabular}{|l|l|}
\hline Kritisches Denken & $\begin{array}{l}\text { Eigene Praktiken, Meinungen, Werte und Normen kritisch } \\
\text { hinterfragen und die Bereitschaft entwickeln, sich damit } \\
\text { auseinanderzusetzen. }\end{array}$ \\
\hline Selbstkompetenz & $\begin{array}{l}\text { Fähigkeit entfalten, über die eigene Rolle in der lokalen } \\
\text { Gemeinschaft und der globalen Gesellschaft zu reflek- } \\
\text { tieren. Aushandlungsprozesse führen, Entscheidungen } \\
\text { treffen, Positionen beziehen, Verantwortung übernehmen } \\
\text { und Reflexionskompetenz entwickeln. Mit einer prospek- } \\
\text { tiven Haltung den rasanten Entwicklungen und Unsicher- } \\
\text { heiten auf konstruktive Weise begegnen und Selbst- } \\
\text { kompetenz wahrnehmen. }\end{array}$ \\
\hline $\begin{array}{l}\text { Integrierte Problemlöse- } \\
\text { kompetenz }\end{array}$ & $\begin{array}{l}\text { Über unterschiedliche Strategien für komplexe Probleme } \\
\text { verfügen und für Lösungen im Sinne einer nachhaltigen } \\
\text { Entwicklung anwenden um praktische Erfahrungen und } \\
\text { Einsichten zu gewinnen. }\end{array}$ \\
\hline
\end{tabular}

Nach Rost (2002, S. 7-12) besteht die pädagogische Herausforderung darin, die Kompetenzen der Lernenden $\mathrm{zu}$ fördern, ihre eigenen Werte und Wertvorstellungen bewusst in ihr Handeln einfließen zu lassen. Diese Kompetenz wird immer wichtiger, weil Lebens- und Entscheidungssituationen aufgrund globaler Vernetzung möglicherweise von widersprechenden Wertvorstellungen gleichzeitig betroffen sind. Diesem Verlangen folgend, sind individuelle Lernvoraussetzungen mit bildungspolitischen und gesellschaftlichen Ansprüchen zu verweben und in konkreten Lernanlässen so $\mathrm{zu}$ realisieren, dass nicht nur anerkannte gesellschaftliche Normen nachvollzogen werden, sondern durch das Erlernen eines Umgangs mit vielen, auch einander widersprüchlichen Wertvorstellungen erreicht werden kann.

In Tab. 2 werden die Rahmenvorgaben und spezifischen Bedingungen zur Realisierung des Dissertationsprojekts skizziert. 
Tab. 2: Elemente für ein Didaktisches Modell für eine nachhaltige Berufsbildung (eigene Darstellung)

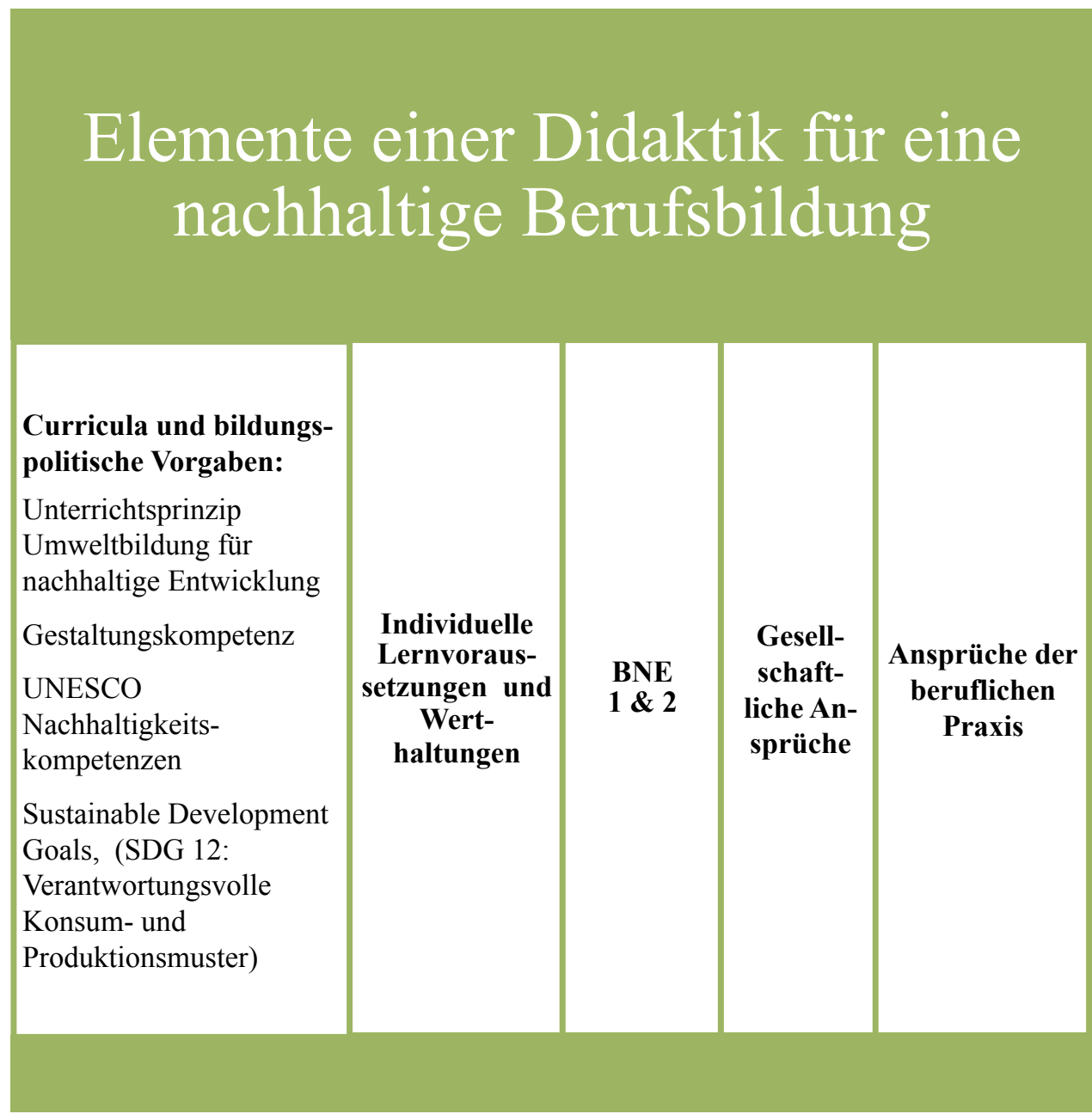

Das didaktische Konzept ,Grüne Pädagogik‘ wurde 2008 an der Hochschule für Agrar- und Umweltpädagogik im Team entwickelt und im Jahr 2013 erstmals öffentlich diskutiert (Wogowitsch, 2013). Durch eine kompetenzorientierte 
Ausrichtung und die Fokussierung auf den Lerntransfer sollen Lernende befähigt werden, ihr Wissen in variablen Situationen anzuwenden.

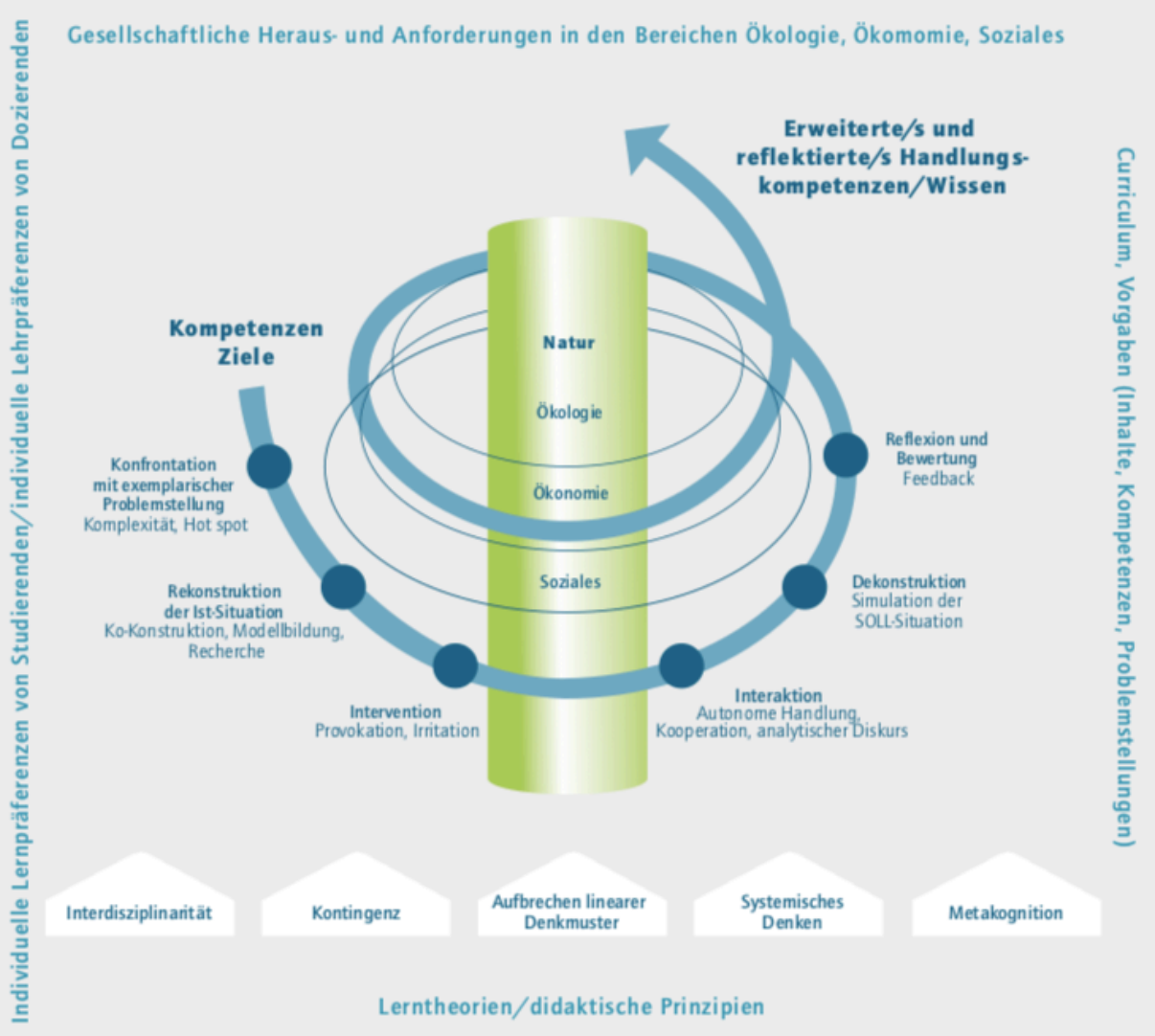

Abb. 3: Die didaktische Leitlinie der Grünen Pädagogik (Forstner-Ebhart \& Haselberger, 2016)

In Anlehnung an Reich (2010) erfolgt der partizipative Aneignungsprozess als Konstruktion, Rekonstruktion und Dekonstruktion: Als Konstruktion durch Bewusstwerdung, Sinnfindung und Selbsterfahrung, als Rekonstruktion durch eine sammelnde und entdeckende Wiedergabe um auf Handlungen bezogene Standards zu erreichen und durch Dekonstruktion um durch zirkuläres und

Kapelari, Suzanne (Hg.), Vierte „,Tagung der Fachdidaktik“ 2019:

„Interdisziplinäre fachdidaktische Diskurse zur Bildung für nachhaltige Entwicklung“

(C) 2020 innsbruck university press, ISBN 978-3-99106-019-2, DOI 10.15203/99106-019-2 
systemisches Vorgehen die Ordnung der Dinge zu hinterfragen und um durch Positionswechsel neue Perspektiven einnehmen $\mathrm{zu}$ können. Eine Auseinandersetzung mit komplexen gesellschaftlich relevanten Themen ist dabei aus unterschiedlichen Perspektiven in einem interdisziplinären Team zu planen und $\mathrm{zu}$ realisieren. Der Wissenserwerb wird als weitgehend selbstorganisierter Aneignungsprozess verstanden, der in hohem Maß systemisch und situativ erfolgt und letztlich von dem kognitiven Vorwissen, den Verarbeitungsprinzipien, Motivationen und Emotionen der Lernenden gesteuert wird. Die Planung von Lernarrangements erfolgt in Bezug auf Ziele, welche kommunikative Kompetenz und in Folge Gestaltungskompetenz determinieren. In einem diskursorientierten Lernsetting werden an sogenannten Hotspots, welche auf Problem-, Konflikt- und Dilemmasituationen abzielen, bestehende Denkkonzepte überprüft und in einem Co-creation Prozess bearbeitet (ForstnerEbhart \& Haselberger, 2016).

Lernprozesse, welche $\mathrm{zu}$ selbstgesteuerten Denk-, Arbeits- und Handlungsweisen verhelfen, analytische Vergleiche und einen Perspektivenwechsel ermöglichen, zum Diskurs und zum Nachdenken anregen, durch pointiert formulierte Aussagen in der Interventionsphase z.B. mittels Storytelling auch einen amüsierenden Charakter annehmen können, die Komplexität reduzieren oder Struktur geben, entsprechen dem Anforderungsprofil einer nachhaltigen Berufsbildung. Im Sinne Luhmanns (2015) wird dadurch ein Kompetenzaneignungsprozess in Gang gesetzt, der über Lernaktivitäten in komplexen Situationen und das Einnehmen unterschiedlicher Rollen auf eine unbekannt bleibende Zukunft vorbereitet und das Nichtwissen bzw. Nichtkönnen als Entwicklungspotenzial begreift (Wogowitsch, 2013). ,Grüne Pädagogik' verlangt nach Methoden für nachhaltig wirksame Bildungsprozesse im Lichte der thematischen Relevanz und der vorhandenen Ressourcen entsprechend der Lernvoraussetzungen und der angestrebten Ziele für die jeweilige Zielgruppe. Für ernährungsbezogene Themen seien beispielhaft Visualisierungen in Form von Produkt- oder Prozesslandkarten, Tagebuchaufzeichnungen (Ernährungstagebuch), Szenarios, diskursive Methoden, Kraftfeldanalysen oder Food Stories erwähnt. Die auf Unterrichtsziele abgestimmte Methodenwahl und Adaption ermöglicht in den

Kapelari, Suzanne (Hg.), Vierte „Tagung der Fachdidaktik“ 2019:

„Interdisziplinäre fachdidaktische Diskurse zur Bildung für nachhaltige Entwicklung“

(C) 2020 innsbruck university press, ISBN 978-3-99106-019-2, DOI 10.15203/99106-019-2 
einzelnen Phasen des didaktischen Handelns Diskurs-, Handlungs-, Entscheidungsräume, wo Lernenden der Zugang zu sozial und kulturell geprägten Werthaltungen und zur realen Lebenswelt eröffnet wird.

Didaktische Leitfragen basierend auf systemisch-konstruktivistischen Kriterien unterstützen die Planung einer den Zielen und den Lernvoraussetzungen der Zielgruppe angemessenen Vorgehensweise in der Planung von Lernsettings (Forstner-Ebhart \& Wogowitsch, 2013) und (Forstner-Ebhart \& Linder, 2019). Daran anknüpfend fragt das Dissertationsprojekt nach den Potenzialen und Schwächen der ,Grünen Pädagogik' im Zusammenhang mit der Entwicklung von Werthaltungen bei SchülerInnen.

\section{Forschung}

\subsection{Forschungsbefunde}

Einleitend werden relevante Forschungsbefunde kurz skizziert, welche Fragen begrenzter Ressourcen zusammenführen. Wie bereits 1987 im Brundtlandbericht (United Nations, 1987) veröffentlicht, wird nach einem Handeln im Bewusstsein globaler Verantwortung verlangt. In der Forschung des berufsbildenden Schulwesens zur BNE gibt es nach Rieckmann (2016) erste Modellversuche $\mathrm{zu}$ den Aspekten nachhaltiger Entwicklung als Wirtschaftsfaktor und Managementstrategie, Energieeffizienz und Ressourcenschonung sowie Nachhaltigkeit in der Berufsbildung, die einer strukturellen Verankerung bedürfen. Im österreichischen Bildungsbericht 2018 (Schlögl, Stock, \& Mayerl, 2019) sind im Kapitel ,Berufliche Erstausbildung' hinsichtlich Zukunftsorientierung lediglich Digitalisierung und Industrie 4.0 erwähnt. Für die Sicherung der Zukunfts- und Innovationsfähigkeit wird jedoch auf eine sektorenübergreifende Zusammenarbeit hingewiesen. Eine Studie (Engweiler, Hasenstab, Schallhart, \& Steinmüller, 2017) zu nachhaltigen und demokratischen Unternehmen ortet umfangreiche Entwicklungsschwerpunkte, wie z.B. in der Fehler- und Lernkultur, in einer partizipativen Entscheidungs-

Kapelari, Suzanne (Hg.), Vierte „Tagung der Fachdidaktik“ 2019:

„Interdisziplinäre fachdidaktische Diskurse zur Bildung für nachhaltige Entwicklung“

(C) 2020 innsbruck university press, ISBN 978-3-99106-019-2, DOI 10.15203/99106-019-2 
kultur oder bei Ressourcen- und Nachhaltigkeitsmanagementsystemen. Grunwald und Kopfmüller (2012) weisen in ihren Ausführungen auf den Gestaltungsanspruch des Nachhaltigkeitsleitbildes in Verbindung mit der Unsicherheit vieler relevanter Wissensbestände hin, der mit dem Erwerb von strategischer und reflexiver Kompetenz einhergeht. Die genannten Forschungsbefunde bilden den Ausgangspunkt für die weitere empirische Arbeit im Dissertationsprojekt.

\subsection{Forschungsinteresse}

Die Idee zu dieser Arbeit entwickelte sich aus der intensiven Mitarbeit bei der Entwicklung der ,Grünen Pädagogik' an der Hochschule für Agrar- und Umweltpädagogik Wien. In der fachdidaktischen Forschung zur Entwicklung von Werthaltungen liegen für die ,Grüne Pädagogik' noch keine Evidenzen vor. Das Forschungsinteresse richtet sich einerseits auf die Beantwortung der Frage, welche Anforderungen an eine nachhaltigkeits- und werteorientierte Didaktik zu stellen sind, um daraus Schlüsse für die Weiterentwicklung zu ziehen und einen Beitrag zur Konkretisierung der Bildung für nachhaltige Entwicklung zu leisten. Es wird untersucht, ob die ,Grüne Pädagogik' bei der Entwicklung von Werthaltungen handlungsleitend wirkt und einen Beitrag zur Erweiterung individueller Handlungsspielräume leistet.

\subsection{Forschungsfragen}

Die Forschungsfragen zielen auf Werthaltungen und Kompetenzen ab, die bei Entscheidungsprozessen zu Nachhaltigkeitsfragen sichtbar werden. Durch die Beantwortung Forschungsfragen wird der Versuch unternommen, Potenzialen und Schwächen der, Grünen Pädagogik' zu beschreiben und Ergebnisse für deren Weiterentwicklung hervorzubringen.

Die zentrale Forschungsfrage bezieht sich auf Werthaltungen der SchülerInnen die in Entscheidungsfindungsprozessen zum Tragen kommen. Es wird untersucht, ob durch eine Unterrichtsgestaltung nach der ,Grünen Pädagogik' 
SchülerInnen die eröffneten Handlungs- und Entscheidungsräume zur Entwicklung von Werthaltungen aufgreifen und welche Werthaltungen SchülerInnen in nachfolgenden Diskussionen zeigen.

Daran schließt die Frage nach den didaktischen Anforderungen an einen Unterricht zur Entwicklung von Werthaltungen.

\subsection{Forschungsmethodik}

Die Untersuchung wurde an einer dreijährigen berufsbildenden Schule in Wien im Zeitraum Oktober 2017 bis Jänner 2019 als ein qualitativ-empirisches Erhebungs- und Auswertungsverfahren durchgeführt. Eingangs wurde der Lehrplan einer systematischen BNE-Kontextanalyse hinsichtlich Themen, Ziele, Kompetenzen und didaktischen Hinweisen unterzogen und mit den Ansprüchen der Sustainable Development Goals in Beziehung gesetzt.

Der Datenerhebung gingen Unterrichtshospitationen, die Teilnahme an einem Schulgartenworkshop mit teilnehmender Beobachtung voraus um Einblicke in den Schulbetrieb zu gewinnen und die SchülerInnen kennen zu lernen. Die dabei gewonnenen Erkenntnisse wurden protokolliert und für die Planung nachfolgender Unterrichtseinheiten nach der didaktischen Leitlinie der , Grünen Pädagogik' im Fachbereich Naturwissenschaften, Betriebsorganisation mit Übungen und in Volkswirtschaft und Wirtschaftsgeografie herangezogen.

Die empirische Untersuchung gliederte sich in zwei Phasen: in der ersten Phase wurden drei Unterrichtsinterventionen im zweiten Jahrgang ( $\mathrm{n}=31 \mathrm{im}$ Alter von 15 bis 16 Jahren, 25 weiblich und 6 männlich) zu Nachhaltigkeitsthemen durchgeführt. Daran anschließend fanden Reflexionsgespräche mit SchülerInnen und Lehrkräften statt. In der zweiten Phase erhielten SchülerInnen eine Einladung zu einer Gruppendiskussion (Billmann-Mahecha \& Gebhard, 2014), wo die Teilnehmenden ( $\mathrm{n}=10,7$ weiblich, 3 männlich) in einer gemeinsam geführten Diskussion in Bezug auf nachhaltige Konsum- und Produktionsmuster verantwortungsvolle Entscheidungen $\mathrm{zu}$ treffen hatten, die über deren gewohnten Verantwortungsbereich hinausgingen. Die den Entscheidungen zugrundeliegenden Werthaltungen wurden mit der

Kapelari, Suzanne (Hg.), Vierte ,Tagung der Fachdidaktik“ 2019:

„Interdisziplinäre fachdidaktische Diskurse zur Bildung für nachhaltige Entwicklung“

(C) 2020 innsbruck university press, ISBN 978-3-99106-019-2, DOI 10.15203/99106-019-2 
Dokumentarischen Methode (Bohnsack, Nentwig-Gesemann, \& Nohl, 2013) computerunterstützt durch das Programm MAXQDA 2018 (Rädiker \& Kuckartz, 2019) analysiert.

Zusätzlich liegen die Ergebnisse einer schriftlichen Befragung der Lehrkräfte $(n=6)$ und die Ergebnisse der Schulwebsite-Recherche hinsichtlich BNERelevanz vor.

Durch die noch ausständige Methodentriangulation nach Flick (2011) soll die Validität der Forschungsergebnisse bestätigt sowie ein vollständigeres Gesamtbild des Forschungsgegenstandes gezeichnet werden. Die Gesamtergebnisse dieser Untersuchung sind zurzeit in Bearbeitung und liegen noch nicht zur Gänze vor.

\subsection{Erste Ergebnisse und Diskussion}

Die Gruppendiskussion der SchülerInnen gewährt Einblicke in Werthaltungen im Kontext der Nachhaltigkeit, die in Aussagen zu Selbstwirksamkeit, Gerechtigkeit, Optimismus, Offenheit, Weitsichtigkeit, Achtsamkeit, Verzicht, Solidarität, Ehrlichkeit und Achtung vor der Natur zum Ausdruck kommen (vgl. dazu unter 1.2, BNE und Werte').

SchülerInnen zeigen in ihren Äußerungen eine patriotische Einstellung gegenüber lokalen, regionalen und nationalen Produkten. Diese Aspekte finden im gesamten Diskussionsverlauf Erwähnung und scheinen eine vertrauensbildende Wirkung bei Konsumentscheidungen auszuüben. Der Agrarsoziologe Markus Schermer beschäftigte sich eingehend mit diesem Phänomen, welches seit dem Jahr 1995, dem EU-Beitritt Österreichs auftritt und als ,Konsumpatriotismus' zum Ausdruck kommt (2015, S. 121-132).

Sie sehen prinzipiell „reiche“ Erwachsene für nachhaltige Konsummuster bzw. den Kauf einheimischer Konsumgüter in der Verantwortung, da durch die begrenzt zur Verfügung stehenden Ressourcen die DiskutantInnen ihren eigenen Handlungsspielraum eingeengt wahrnehmen. Sie sind der Meinung zu wenig Kaufkraft zu besitzen, um einen entscheidenden Beitrag für eine nachhaltige Entwicklung zu leisten, wie z.B. teurere Bioprodukte kaufen „....Menschen mit 
Geld, nicht so wie wir, .... ja weil wir das halt eben nicht haben “. SchülerInnen stellen in allen Passagen der Diskussion Bezüge zwischen sozialem Status, nachhaltigem Handeln, Klimafolgen und globaler Verantwortung her und sind sich der Auswirkungen ihrer Konsumentscheidungen bewusst „... du musst auch irgendwie an andere Menschen denken ... ".

Mit zunehmender Entfernung zwischen Produktions- und Konsumort steigt bei SchülerInnen die Skepsis hinsichtlich Qualität, gerechter Entlohnung oder fairen Arbeitsbedingungen der Mitarbeiter. Gerechtigkeit ist jener Wert, der im Zusammenhang mit internationalem Handel besonders oft erwähnt wird und eine besonderes hohe Zustimmung erfährt ,Sonnentor ist nachhaltig, die müssen fair bezahlen, oder?"

In den Entscheidungsfindungsprozessen werden Argumente aus dem sozialen Umfeld mit im Unterricht behandelten Themenbereichen in Beziehung gesetzt und diskutiert, wie z.B. ,Food Waste', wo Konzepte für die Verwertung von Altbrot mit den im Unterricht diskutierten Wegen zur Reduktion von Lebensmittelabfällen genannt und neu verknüpft wurden. Dem gesamten Lebensmittelhandel wird wegen des wettbewerbsbedingten Überangebots von Lebensmitteln eine geringe Solidarität zugeschrieben „... bis um 20.00 Uhr so eine volle Brottheke sehen mit frisch gebackenem Brot, das ist ja auch wieder abartig! ".

In allen Diskussionsabschnitten wurde der hohe Ressourceneinsatz für den Warentransport kritisiert und Unverständnis gegenüber Warenströmen zum Ausdruck gebracht ,... der Treibstoff der verbraucht wird, wenn man etwas exportiert, das ist ja auch wieder mal nicht nachhaltig ... “, ,,... warum kaufen wir Tomaten aus Spanien, wenn wir selber Tomaten haben... “. SchülerInnen beschreiben eine umweltverträgliche Produktion als lokal unter Achtung der Natur produzierte Ware, welche auf kurzem Weg über den Handel die Endverbraucher erreicht.

Der Kommunikationsprozess dokumentiert die soziale Prägung durch die familiäre Situation und den Einfluss der Schule auf die Entwicklung von Werthaltungen. Erfahrungen mit Alkohol- oder Drogenkonsum, Einkauf am Bauernhof oder Lebensmittelbeschaffung auf Selbsternteflächen lassen auf Erfahrungen aus dem sozialen Umfeld schließen „... Hanftee ist ja nichts

Kapelari, Suzanne (Hg.), Vierte „Tagung der Fachdidaktik“ 2019:

„Interdisziplinäre fachdidaktische Diskurse zur Bildung für nachhaltige Entwicklung“

(C) 2020 innsbruck university press, ISBN 978-3-99106-019-2, DOI 10.15203/99106-019-2 
Schlimmes ..., ... wer geht heutzutage schon wirklich jedes Jahr Erdbeeren pflücken?" Fachliche Kompetenz in Verbindung mit Achtsamkeit beim Umgang mit Lebensmitteln über das Ablaufdatum hinaus kommt in einer Diskussionspassage zum Ausdruck ,...Eier werden wirklich schlecht ... bei Sachen wo man eigentlich schon weiß, dass es lange hält, aber da steht, es hält gar nicht so lange ... könnte man verarbeiten ...", was auf eine hohe fachliche Kompetenz betreffend Lebensmittelkennzeichnung, Haltbarkeit und Achtsamkeit im Umgang mit dem Ablaufdatum schließen lässt. In der Diskussion werden Beispiele für die Verarbeitung von Lebensmitteln, die kurz vor dem Verderb stehen, genannt und beschrieben, wie z.B. Bananenmuffins. Schülerinnen zeigen in der Diskussion Verständnis auch für nicht nachhaltige Konsummuster, wenn diese in geringem Haushaltsbudget begründet liegen. Er zeigt sich in kontroversen Diskurspassagen ,,...die Arbeitslosigkeit steigt immer mehr, es steigt die Armut immer mehr natürlich, dass die Menschen zu billigeren Produkten greifen ... “.

Eine optimistische Haltung für eine nachhaltige Entwicklung kommt durch die Wertschätzung innovativer Ideen bei Recyclingprodukten zum Ausdruck ,,... es ist halt auch wieder eine gute Idee, Sachen nochmal zu bearbeiten anstatt wieder neu zu produzieren ... ", die Gedanken finden eine Fortsetzung, indem in der Diskussionsrunde Ideen für weitere Recyclingprodukte genannt werden „,man könnte es dann ausbauen, auf mehrere Produkte...man kann auch andere Produkte herstellen “.

Generell lässt sich aus allen Äußerungen der SchülerInnen ein hohes Misstrauen gegenüber international agierenden Unternehmen hinsichtlich deren ökonomischen Interessen und der Ausbeutung von MitarbeiterInnen ableiten.

SchülerInnen zeigen sich gegenüber einer Gewinnbeteiligung von Fischern als Zulieferer von Fischernetzen für die Herstellung von Skateboards solidarisch und betrachten es als ihre Verantwortung, mutig für den Schutz der Meere vor Verunreinigung aufzutreten „Ja, ich finde das mit den Skateboards am besten, weil Leute die das Skateboard kaufen wollen aber auch die Fischer bekommen dadurch auch mehr Geld! ".

Kapelari, Suzanne (Hg.), Vierte „Tagung der Fachdidaktik“ 2019:

„Interdisziplinäre fachdidaktische Diskurse zur Bildung für nachhaltige Entwicklung“

(C) 2020 innsbruck university press, ISBN 978-3-99106-019-2, DOI 10.15203/99106-019-2 
Subjektive Theorien, wie z.B. „... alle Äpfel werden einmal ranzig“ führen zu Fehlinterpretationen hinsichtlich der Bewertung von Qualität und Haltbarkeit bei Biolebensmitteln und konventionell erzeugten Produkten. Solche Aussagen stellen einen wertvollen Impuls für eine mehrperspektivische Auseinandersetzung mit dem Themenbereich ,Umweltverträgliche Produktion' dar.

Durchgängig findet der Schulgarten als naturnaher Lebensraum, als Ort, wo man seine Freizeit in angenehmer Atmosphäre verbringen kann und wo in Gemeinschaft gearbeitet bzw. gelernt wird Erwähnung ,,... aber natürlich freut man sich, wenn andere mitspielen und man sich auch mit den Leuten gut versteht... “. Die positive Einstellung der SchülerInnen für den Schulgarten könnte auf die von den Lehrkräften forcierte Nutzung als Outdoorklasse für den Sport- und Biologieunterricht zurückzuführen sein. Pflanz-, Pflege- und Erntearbeiten am Hochbeet und an der Kräuterschnecke sowie die Verarbeitung der Erträge in der Küchenpraxis sind als Begründung für das vorhandene Wissen um Vielfalt, saisonale und regionale Verfügbarkeit und den Wert der selbst erzeugten Lebensmittel naheliegend.

Die vorliegenden Ergebnisse betreffend Unterrichtsorganisation weisen auf einen Optimierungsbedarf hinsichtlich der erforderlichen zeitlichen Ressourcen für die Entwicklung von Werthaltungen hin. Die drei angebotenen Unterrichtssettings nach der, Grünen Pädagogik' gewährten durch den zirkulär angeordneten Lernprozess und durch partizipative Arbeitsformen unter Lehrenden und Lernenden umfangreiche Möglichkeiten, an vorherrschenden Stereotypen zu arbeiten und eine nachhaltige Entwicklung zu fördern.

Die Befragung der Lehrkräfte zeigt ein uneinheitliches Verständnis von Nachhaltiger Bildung, nachhaltigem Handeln und betreffend SchülerInnenpartizipation.

Die Recherche der Schulwebsite ergab ein klares Bekenntnis für eine nachhaltige Bildung und $\mathrm{zu}$ nachhaltigem Handeln. Durch ein auf Nachhaltigkeit ausgerichtetes Leitbild für den Fachbereich ,Ernährungswirtschaft' und durch mehrfache Umweltzertifizierungen der Schule kommt dieses Anliegen zum Ausdruck. In der Schulküchenverpflegung bzw. dem Schulbuffet realisiert sich die im Leitbild beschriebene Haltung und drückt sich sowohl bei Lehrenden als bei SchülerInnen durch die hohe Wertschätzung

Kapelari, Suzanne (Hg.), Vierte „Tagung der Fachdidaktik“ 2019:

„Interdisziplinäre fachdidaktische Diskurse zur Bildung für nachhaltige Entwicklung“

(C) 2020 innsbruck university press, ISBN 978-3-99106-019-2, DOI 10.15203/99106-019-2 
gegenüber dem vorwiegend vegetarischen Angebot aus ,,das Jausenbuffet ist super, weil es da immer frische Sachen gibt “.

\section{Zusammenfassung/Ausblick}

Eine nachhaltig wirksame und werteorientierte Lernkultur kann entstehen, wenn im Sinne der Gestaltungskompetenz Raum für persönliche Entfaltung geschaffen, wahrgenommen und Selbstwirksamkeit erlebt werden.

Eine strukturierte Herangehensweise und systemisches Denken nach dem didaktischen Modell ,Grüne Pädagogik' gewährleisten Reflexions- und Diskurspotenziale für die Mensch-Umwelt-Beziehung und eröffnen Handlungsspielräume für den Aufbau moralisch-ethischer und beruflicher Kompetenzen. Fachwissen im Kontext von BNE und nachhaltiges Handeln im Beruf und im sozialen Umfeld ermöglichen Lernenden zu Fragen der Solidarität und Gerechtigkeit Stellung zu beziehen und so zu GestalterInnen im Sinne einer zukunftsfähigen Entwicklung zu werden.

Eine schulorganisatorische Neuorientierung hinsichtlich Unterrichtsblockung für längerfristige Lern- und Arbeitseinheiten in interdisziplinären Teams scheint für die Umsetzung einer nachhaltigen Bildung unverzichtbar zu sein und bedarf weiterer Forschungen hinsichtlich Struktur und Kooperation sowie Partizipation aller Beteiligten.

Der Entwicklung von Werthaltungen kommt als integraler Bestandteil einer nachhaltigen Bildung eine wichtige Rolle zu. Die ,Grüne Pädagogik' ermöglicht durch den zirkulär angeordneten Prozess des Lernens moralische Haltungen und Überzeugungen bewusst $\mathrm{zu}$ machen, Konflikte früh wahrzunehmen und berufliches wie privates Handeln an ethischen Überzeugungen auszurichten.

Der mit dem Aufsatz eröffnete Diskurs soll dazu beitragen, Werthaltungen, im Kontext einer emanzipatorischen BNE für Lernende, Lehrende systematisch zu beschreiben und darüber den Diskurs zu eröffnen. 


\section{Literatur}

Ökobüro-Allianz der Umweltbewegung. (2017). SDG 12: Nachhaltige/r Konsum und Produktion. SDG Watch Austria. Wien.

Österreichischer Austauschdienst. (2014). Das österreichische Bildungssystem. (Ö. Austauschdienst-GmbH, Herausgeber) Abgerufen am 07. 012020 von Euroguidance Österreich: https://www.bildungssystem.at/footer-boxen/iscedklassifikation/internationale-standardklassifikation-im-bildungswesen/

Billmann-Mahecha, E., \& Gebhard, U. (2014). Die Methode der Gruppendiskussion zur Erfassung von Schülerperspektiven. In D. Krüger, I. Parchmann, \& H. H. Schecker, Methoden in der naturwissenschaftsdidaktischen Forschung (S. 147158). Berlin, Heidelberg: Springer.

Bohnsack, R., Nentwig-Gesemann, I., \& Nohl, A.-M. (2013). Die dokumentarische Methode und ihre Forschungspraxis (Bd. 3.). (A.-M. Nohl, Hrsg.) Wiesbaden: Springer.

Bundesministerium für Bildung und Frauen. (2014). Grundsatzerlass Umweltbildung für nachhaltige Entwicklung. Grundsatzerlass Umweltbildung für nachhaltige Entwicklung. (B. f. Frauen, Hrsg.) Wien: Digitales Druckzentrum Renngasse.

Bundesministerium für Bildung und Frauen. (2015). Bildungsstandards. Abgerufen am 07. 012020 von

https://www.bildungsstandards.berufsbildendeschulen.at/kompetenzorientiertes -unterrichten: www.bildungsstandards.berufsbildendeschulen.at

Bundesministerium für Bildung, W. u. (2015). Berufsbildende Schulen in Österreich, 2019. Abgerufen am Juli 2019 von https://www.abc.berufsbildendeschulen.at/downloads/?kategorie $=10$

De Haan, G. (2002). Die Kernthemen der Bildung für eine nachhaltige Entwicklung. Zeitschrift für internationale Bildungsforschung und Entwicklungspolitik, 25. Nürnberg: Verlag für Interkulturelle Kommunikation.

Deutsche UNESCO-Kommission. (2014). UNESCO Roadmap zur Umsetzung des Weltaktionsprogramms Bildung für nachhaltige Entwicklung". UNESCO, Nachaltige Bildung. Bonn: Brandt.

Deutsche UNESCO-Kommission. (2014). UNESCO Roadmap zur Umsetzung des Weltaktionsprogramms Bildung für nachhaltige Entwicklung". Deutsche UNESCO-Kommission. Bonn: Brandt.

Engweiler, C., Hasenstab, C., Schallhart, A., \& Steinmüller, B. (2017). Studie zu nachhaltigen und demokratischen Unternehmen. Wien.

Flick, U. (2011). Triangulation. Eine Einführung (3. Ausg.). Wiesbaden: VS Verlag Springer.

Kapelari, Suzanne (Hg.), Vierte „Tagung der Fachdidaktik“ 2019:

„Interdisziplinäre fachdidaktische Diskurse zur Bildung für nachhaltige Entwicklung“

(C) 2020 innsbruck university press, ISBN 978-3-99106-019-2, DOI 10.15203/99106-019-2 
Forstner-Ebhart, A., \& Haselberger, W. (2016). Das Theoriefundament der Grünen Pädagogik. In F. u.-u. Vizerektorat für Umweltpädagogik, Grüne Pädagogik Türöffner zu nachhaltigem Lernen. Wien: Janecek.

Forstner-Ebhart, A., \& Linder, W. (2019). Nachhaltigkeitsbildung als Brücke zwischen Allgemeinbildung und Berufsbildung. In L. Kirner, B. Stürmer, \& E. Hainfellner, Einblicke in ausgewählte Forschungsfelder der Agrar- und Umweltpädagogik (Bd. 1). Innsbruck: Studienverlag.

Forstner-Ebhart, A., \& Wogowitsch, C. (2013). Planung von Lernszenarien. In F. u. Vizerektorat für Umweltpädagogik. Wien: AV Astoria.

Frei, E., Iwaniewicz, P., Pfaffenwimmer, G., Steiner, R., \& Streissler, A. (2014). UNECE-Report 2014. Zusammenfassung und Endbericht. Österreichisches Bundesministerium für Wissenschaft, Forschung und Wirtschaft, Bildung für Nachhaltige Entwicklung. Wien: Eigenverlag. Abgerufen am Juli 2019

Frey, D. (2016). Psychologie der Werte. Berlin, Heidelberg: Springer.

Fritz, U., \& Willenshofer, K. (2012). Bundesministerium für Bildung, Wissenschaft und Forschung. Abgerufen am Juli 2019 von

https://www.bildungsstandards.berufsbildendeschulen.at/sites/default/files/files /BBS-Bildungsstandards-KU-Grundlagenpapier-16.07.2012.pdf

Grunwald, A., \& Kopfmüller, J. (2012). Nachhaltigkeit (2. Ausg.). Frankfurt, New York: Campus.

Hoeckel, K. (2010). Learning for Jobs OECD Reviews of Vocational Education and Training. (OECD, Hrsg.) Abgerufen am Juli 2019 von

http://www.oecd.org/education/45407970.pdf

Industrie- und Handelskammer Nürnberg für Mittelfranken. (2015). Lexikon der Nachhaltigkeit. (I. u. Mittelfranken, Produzent, \& Aachener Stiftung Kathy Beys) Abgerufen am 07. 012020 von

https://www.nachhaltigkeit.info/artikel/index of sustainable economic welfar e 1874.htm

Iwaniewicz, P., Pfaffenwimmer, G., \& Frei, E. (2008). Österreichische Strategie zur Bildung für nachhaltige Entwicklung. (U. u. Bundesministerium für Land- und Forstwirtschaft, K. u. Bundesministerium für Unterricht, \& B. f. Forschung, Hrsg.) Wien: Eigenvervielfältigung.

Kocher, B. (2016). Bau-, Verkehrs- und Energiedirektion des Kantons Bern Amt für Umweltkoordination und Energie. Abgerufen am Juli 2019 von https://www.bve.be.ch/bve/de/index/direktion/ueber-diedirektion/dossiers/nachhaltige entwicklungne.assetref/dam/documents/BVE/A UE/de/aue ne praktikumsarbeit kocher ne kantone d.PDF

Kyburz-Graber, R., Kunz, M., Dal Cero, M., Spiess, H., Carabias-Hütter, V., Hohl, U., \& Iseli, P. (2006). Kompetenzen für die Zukunft. Bern: Hep. 
Luhman, N. (2015). Soziale Systeme. GRundriss einer allgemeinen Theorie (16. Ausg.). Frankfurt am Main: Suhrkamp.

Niebert, K. (2016). Nachhaltigkeit lernen im Anthropozän. In M. K. Schweer, \& M. K. Schweer (Hrsg.), Bildung für nachhaltige Entwicklng in pädagogischen Handlungsfeldern (S. 77-94). Frankfurt am Main: Peter Lang.

OECD, Bildung, Abteilung Bildungs- und Ausbildungspolitik. (2019). OECD. Abgerufen am 07. 012020 von https://www.oecd.org/austria/47514978.pdf

Payrhuber, A. (2013). Grüne Pädagogik und Werte. In C. Wogowitsch, C. Wagner-Alt, K. Klement, \& V. f.-u. Wien (Hrsg.), Zugänge. Forschungs- und Entwcklungsbericht der Hochschule für Agrar- und Umweltpädagogik. Wien: $\mathrm{AV}+$ Astoria.

Rädiker, S., \& Kuckartz, U. (2019). Analyse qualitativer Daten mit MAXQDA. Text, Audio und Video. Wiesbaden: Springer.

Reich, K. (2010). Erfinder, Entdecker und Enttarner der Wirklichkeit. Das systemischkonstruktivistische Verständnis von Lernen und Lehren. Pädagogik, S. 42-47.

Republik Österreich. (2013). Rechtsinformationssystem des Bundes. Abgerufen am 07. 012020 von https://www.ris.bka.gv.at/eli/bgbl///2013/111

Rieckmann, M. (2016). Bildung für nachhaltige Entwicklung - Konzeptionelle Grundlagen und Stand der Implementierung. In M. Schweer, \& M. Schweer (Hrsg.), Bildung für nachhaltige Entwicklung in pädagogischen Handlungsfeldern. Frankfurt/Main: Peter Lang.

Rieckmann, M. (2016). Bildung für nachhaltige Entwicklung-Konzeptionelle Grundlagen und Stand der Implementierung. In M. Schweer, Bildung für nachhaltige Entwicklung in pädagogischen Handlungsfeldern. Grundlagen, Verankerung und Methodik in ausgewählten Lehr-Lern-Kontexten (S. 11-32). Frankfurt/Main: Peter Lang.

Rieckmann, M. (2016). Kompetenzentwicklungsprozesse in der Bildung erfassen: Überblick über ein heterogenes Forschungsfeld. In M. Barth, \& $\mathrm{M}$. Rieckmann, Empirische Forschung zur Bildung für nachhaltige Entwicklung Themen, Methoden und Trends. Opladen, Berlin, Toronto: Barbara Budrich .

Rieckmann, M. (2019). Beiträge einer Bildung für nachhaltige Entwicklung zum Erreichen der Sustainable Development Goals-Perspektiven, Lernziele und Forschungsbedarfe. In M. Rieckmann, I. Clemens, S. Hornberg, \& M. Rieckmann (Hrsg.), Bildung und Erziehung im KOtext globaler Transformation. opladen, Berlin, Toronto: Barbara Budrich.

Rieckmann, M. (2019). Kompetenzen für eine nachhaltige Entwicklung - konzeptionelle Präzisierung der Nachhaltigkeitskompetenz über den Leistungsanspruch. In I. Clemens, S. Hornberg, \& M. Rieckmann, Bildung und Erziehung im KOntext globaler Transformationen. Opladen, Berlin, Toronto: Barbara Budrich.

Kapelari, Suzanne (Hg.), Vierte „Tagung der Fachdidaktik“ 2019:

„Interdisziplinäre fachdidaktische Diskurse zur Bildung für nachhaltige Entwicklung“

(C) 2020 innsbruck university press, ISBN 978-3-99106-019-2, DOI 10.15203/99106-019-2 
Rieckmann, M., Fischer, D., \& Richter, S. (2014). Nachhaltige Ernährung im Wertediskurs-Beiträge einer Hochschulbildung für nachhaltige Entwicklung. In C. Schank, K. Vorbohle, \& J. Quandt, Perspektive Nahrungsmittelethik (S. 29-58). München: Rainer Hampp.

Robinsohn, S. B. (1971). Bildungsreform als Revision des Curriculum und ein Strukturkonzept für Curriculumentwicklung. Neuwied: Luchterhand.

Rockstroem, J., Steffen, W., Richardson, K., Cornell, S., \& Fetzer, I. (2015). American Association for the Advancement of Science. Abgerufen am Juli 2019 von https://science.sciencemag.org/content/347/6223/1259855.full

Rost, J. (2002). Umweltbildung - Bildung für nachhaltige Entwicklung. Was macht den Unterschied. Zeitschrift für internationale Bildungsforschung, 25. Nürnberg: Verlag für Interkulturelle Kommunikation.

Schermer, M. (2015). From "Food from Nowhere" to "Food from Here:" changing producer-consumer relations in Austria. . Agriculture and Human Values, 32, S. 121-132.

Schlögl, P., Stock, M., \& Mayerl, M. (2019). Berufliche Erstausbildung: Herausforderungen und Entwicklungsaufgaben in einem bedeutsamen Bildungssegment Österreichs. In S. Breit, F. Eder, K. Krainer, C. Schreiner, A. Seel, \& C. Spiel, Nationaler Bildungsbericht Österreich 2018, Band 2. (Bd. 2, S. 269-305). Graz: Leykam.

Standop, J. (2016). Werte in der Schule (2. Ausg.). Weinheim und Basel: Beltz.

Steiner, R., \& Rauch, F. (2013). Grundsatzpapier zur Bildung für Nachhaltige entwicklung in der PädagogInnenbildung Neu. Bundesministerium für Wissenschaft und Forschung, Nachhaltige Bildung. Wien: Eigenvervielfältigung.

Stiftung Zukunftsrat und Baustelle Zukunft. (2018). Allgemeine Didaktik einer Bildung für Nachhaltige Entwicklung für Lehrerinnen und Lehrer. (S. Z. Zukunft, Hrsg.) Chur: Edition Rüegger im Somedia Buchverlag.

UNESCO. (2017). Education for Sustainable Development Goals. Learning Objectives. Paris. Von https://unesdoc.unesco.org/ark:/48223/pf0000247444 abgerufen

United Nations. (1987). Report of the World Commission on Environment and Development: Our Common Future. Oslo.

United Nations. (2019). Sustainable Development Goals-Knowledge Platform. Abgerufen am Juli 2019 von https://sustainabledevelopment.un.org/sdg12

Veis, I. (2017). Bundesministerium für Bildung, Wissenschaft und Forschung. Abgerufen am Juli 2019 von

https://www.bildungsstandards.berufsbildendeschulen.at/sites/default/files/bros chuere/BBS-Bildungsstandards-Broschuere-Forstwirtschaft-HLFS.pdf

Vereinte Nationen. (2015). United Nations. Abgerufen am 2019 von https://www.un.org/Depts/german/gv-70/band1/ar70001.pdf

Kapelari, Suzanne (Hg.), Vierte „Tagung der Fachdidaktik“ 2019:

„Interdisziplinäre fachdidaktische Diskurse zur Bildung für nachhaltige Entwicklung“

(C) 2020 innsbruck university press, ISBN 978-3-99106-019-2, DOI 10.15203/99106-019-2 
Wogowitsch, C. (2013). Grüne Pädagogik. Vom Theoriefundament bis zu professionsorientierten Lernarrangements. Hochschule für Agrar- und Umweltpädagogik Wien. Wien: AV-Astoria.

Wogowitsch, C. (2013). Methoden für kreative Lernprozesse. In F. u.-u. Vizerektorat für Umweltpädagogik. Wien: AV Astoria.

Wogowitsch, C. (2016). Wie die Grüne Pädagogik laufen lernte. In W. u. Vizerektorat für Umweltpädagogik, Grüne Pädagogik - Türöffner zu nachhaltigem Lernen. Wien: Janetschek. 


\section{Transformative Bildung im naturwissenschaftlichen Unterricht}

Hochschulprof. Dr. Thorsten Kosler, Pädagogische Hochschule Tirol

Im Diskurs um Bildung für nachhaltige Entwicklung wurde immer wieder die Forderung erhoben, Lernende müssten in die Lage versetzt werden, unser Denken in grundlegender Weise zu hinterfragen und zu verändern (Sterling, 2001; 2003; Scott \& Gough 2003; Vare \& Scott 2007; Wals 2010; Wals \& Dillon 2013). Entsprechend wird im Positionspapier zur Umsetzung von SDG $4^{1}$ in Österreich des Fachbeirates „Transformative Bildung/ Global Citizenship Education“ der Österreichischen UNESCO-Kommission (2019) das Ziel 4 der Agenda 2030 als Auftrag für eine transformative Bildung gesehen (ebd., S. 2). Mit transformativer Bildung ist demnach eine Bildung gemeint, die zu einer notwendigen sozial-ökologischen gesellschaftlichen Transformation beiträgt (ebd., S. 14). Eine solche Bildung erfordere eine eigene Pädagogik, die im Anschluss an Singer-Bodrowski (2016a, 2016b) anhand von drei Merkmalen charakterisiert werden könne: Transformative Bildung zielt auf den Wandel von individuellen „Bedeutungsperspektiven“, versteht sich als kollektiver Bewusstwerdungs- und Emanzipationsprozess und fragt auch nach der notwendigen Veränderung von Kulturen und Strukturen (des Bildungswesens), die emanzipatorisches Lernen behindern (ebd., S. 14). Unklar bleibt an dieser

1 Mit ,SDG 4“ ist das Sustainable Development Goal 4 („Inklusive, gleichberechtigte und hochwertige Bildung gewährleisten und Möglichkeiten lebenslangen Lernens für alle fördern") aus der Agenda 2030 (vgl. dazu Rieckmann in diesem Band) gemeint. 
Stelle, wie der Wandel individueller Bedeutungsperspektiven als individueller Lernprozess mit der Transformation kollektiver Denkstrukturen im Kontext einer nachhaltigen Entwicklung zusammenhängt. Aus meiner Sicht liegt hier ein Theoriedefizit in der Debatte um Bildung für nachhaltige Entwicklung vor. Solange unklar ist, inwiefern individuelle Lernprozesse $\mathrm{zu}$ einer gesellschaftlichen Transformation im Sinne einer nachhaltigen Entwicklung beitragen können, bleibt auch unklar, wie und was die einzelnen Unterrichtsfächer zu eben dieser Transformation beitragen können.

Um diesen Zusammenhang näher zu beleuchten, werden im Kapitel 1 drei Theorieansätze transformativer Bildung daraufhin analysiert, welchen Ertrag sie für die Klärung dieses Zusammenhanges bieten. Als Erstes wird der Ansatz Sterlings im Kontext seiner sustainability education analysiert, da Sterling innerhalb der internationalen Debatte um ,Education for Sustainable Development‘ der Einzige ist, der sich dieser Frage detailliert gewidmet hat. In der Regel wird dieser Frage gar nicht im Detail nachgegangen, sondern es wird dazu auf den in der angelsächsischen Erwachsenenbildung verbreiteten Ansatz des transformativen Lernens verwiesen (vgl. Wals \& Dillon, 2013). Daher wird auch dieser Ansatz dargestellt und analysiert. Da beide Ansätze aus meiner Sicht den Zusammenhang zwischen individuellem Lernen und gesellschaftlicher Transformation nur unbefriedigend klären können, wird als drittes der Ansatz transformatorischer Bildung aus der deutschsprachigen Bildungstheorie dargestellt und gezeigt, inwiefern dieser Ansatz weiter führt. In Kapitel 2 wird dann auf dieser Basis beispielhaft eine Möglichkeit erläutert, wie individuelles Lernen im naturwissenschaftlichen Unterricht so gestaltet werden kann, dass es als Beitrag zu einer gesellschaftlichen Transformation im Kontext einer nachhaltigen Entwicklung gesehen werden kann.

\section{Konzeptionen transformativer Bildung}

Die Idee, Bildung müsse zu einer gesellschaftlichen Transformation beitragen, schließt letztlich direkt an eines der Gründungsdokumente der Debatte um

Kapelari, Suzanne (Hg.), Vierte „Tagung der Fachdidaktik“ 2019:

„Interdisziplinäre fachdidaktische Diskurse zur Bildung für nachhaltige Entwicklung“

(C) 2020 innsbruck university press, ISBN 978-3-99106-019-2, DOI 10.15203/99106-019-2 
Bildung für nachhaltige Entwicklung, dem Artikel 36 der Agenda 21, an. Dort wurde die Idee formuliert, dass hinausgehend über Bindestrich-Pädagogiken eine Neuausrichtung des gesamten Bildungssystems nicht mehr an wirtschaftlichem Wachstum, sondern an nachhaltiger Entwicklung notwendig sei (Hopkins, 2012, S. 23ff.). In die drei im Folgenden dargestellten Konzeptionen transformativer Bildung sind jeweils eine Vielzahl von theoretischen Ansätzen eingeflossen, mit denen die jeweiligen Autoren versucht haben, Details ihrer Konzeptionen auszuarbeiten. Zwei Theorieansätze finden sich dabei in allen drei Konzeptionen und bieten bereits eine erste Möglichkeit, den Zusammenhang von individuellem Lernen und der Transformation kollektiver Denkstrukturen zu beleuchten. Das ist zum einen die Idee des Paradigmenwechsels von Kuhn (1976) und zum anderen die Lerntheorie von Bateson (1987). Im Anschluss an diese Ansätze von Kuhn und Bateson (1.1) wird zunächst das transformative Lernen nach Sterling (1.2), das transformative Lernen aus der angelsächsischen Erwachsenenbildung (1.3) und der transformatorische Bildungsbegriff der deutschsprachigen Bildungstheorie (1.4) vorgestellt und im Hinblick auf den Zusammenhang zwischen individuellem Lernen und kollektivem Umdenken analysiert.

\subsection{Kuhns Paradigmenwechsel und Batesons Lerntheorie}

Kuhn (1976) unterscheidet in seinem Modell von der Struktur wissenschaftlicher Revolutionen als Paradigmenwechsel zwei Phasen des Wandels in der Naturwissenschaft. In Phasen der Normalwissenschaft besteht der Wandel darin, neue Gesetze zu entdecken, die das bestehende Wissensgebäude um einen weiteren Baustein ergänzen, ohne dass es dabei zu Veränderungen von Grundbegriffen oder der Art und Weise kommt, wie die Welt betrachtet wird. In Phasen wissenschaftlicher Revolution werden dagegen Entdeckungen gemacht, die mit den traditionellen Begriffen nicht in Einklang zu bringen seien. Kuhn gibt in einer Zusammenfassung seines Modells (Kuhn, 1981, S. 32f.) drei Charakteristika an, die revolutionäre Veränderungen in seinem Sinne miteinander gemein haben: Erstens haben sie eine systemische

Kapelari, Suzanne (Hg.), Vierte „Tagung der Fachdidaktik“ 2019:

„Interdisziplinäre fachdidaktische Diskurse zur Bildung für nachhaltige Entwicklung“

(C) 2020 innsbruck university press, ISBN 978-3-99106-019-2, DOI 10.15203/99106-019-2 
Struktur, insofern sie nicht etappenweise, Schritt für Schritt, vollzogen werden können, da eine Anzahl untereinander verbundener Gesetzmäßigkeiten revidiert wird. Zweitens vollziehe sich dabei ein Bedeutungswandel, insofern die „Art und Weise, wie Worte und Sätze mit den Naturphänomenen verbunden sind“ (ebd., 34), sich verändert. Und drittens vollzieht sich nach Kuhn ein „grundlegender Wandel des Modells, der Metapher oder der Analogie“ (ebd., S. 36), der für die Mitglieder einer Forschungsgemeinschaft leitend ist (vgl. Kosler, 2016, S. 17-20). Kuhns Arbeit hat in der Wissenschaftstheorie eine umfangreiche Kontroverse ausgelöst und auch die Rezeptionsgeschichte in der Naturwissenschaftsdidaktik ist komplex (Matthews, 2004). Aber die Grundidee, die viele ErziehungswissenschaftlerInnen inspiriert hat, ist wohl die folgende: Wenn ausgerechnet in den vermeintlich, exakten' Naturwissenschaften ein revolutionärer Umbruch im Denken einer Forschungsgemeinschaft möglich ist, dann könnte dies bedeuten, dass ein solch plötzlicher Umbruch des Denkens auch im lernenden Individuum möglich ist. Letztlich setzt Kuhns Theorie des kollektiven Umdenkens sogar voraus, dass ein solch plötzliches Umdenken in Individuen möglich ist, da das kollektive Umdenken ja gerade darin besteht, dass in kurzer Zeit ein relevanter Anteil an Mitgliedern einer Forschungsgemeinschaft ein solches Umdenken individuell vollzieht. In der Naturwissenschaftsdidaktik war Kuhns Ansatz die Inspirationsquelle für die Idee des Conceptual Change, also eines ebenso plötzlichen Bedeutungswandels, der sich im Denken von Lernenden vollzieht. Dazu haben Posner, Strike, Hewson und Gertzog (1982) einige der Kriterien, die Kuhn dafür angibt, unter welchen Bedingungen sich ein solcher Umbruch in Forschungsgemeinschaften vollzieht, auf die Situation von lernenden Individuen übertragen. Auf die Frage, wie ein neues Paradigma inhaltlich zustande kommt, sagt Kuhn nichts. Er beschreibt lediglich Bedingungen, die in Forschungsgemeinschaften erfüllt sein müssen, damit sich ein neues Paradigma in einer Forschungsgemeinschaft gegenüber dem bestehenden durchsetzen kann. Damit bleibt auch unklar, wie ein individueller Lernprozess zu einem kollektiven Umdenken beitragen kann.

Bateson (1987) fasst seine Stufen des Lernens zunächst recht abstrakt. Lernen betrachtet er als Veränderung einer Reaktion auf einen Stimulus. Er geht davon

Kapelari, Suzanne (Hg.), Vierte „Tagung der Fachdidaktik“ 2019:

„Interdisziplinäre fachdidaktische Diskurse zur Bildung für nachhaltige Entwicklung“

(C) 2020 innsbruck university press, ISBN 978-3-99106-019-2, DOI 10.15203/99106-019-2 
aus, dass Organismen den Strom an Erfahrungen und Handlungen im Leben in Abschnitte unterteilen, sie miteinander vergleichen und sie als gleichartig oder verschiedenartig klassifizieren. Solche Abschnitte fasst Bateson als Kontexte auf. Zudem unterscheidet Bateson zwei Ordnungen von Fehlern: Ein Organismus kann Informationen korrekt nutzen, die ihm verraten, aus welcher Menge von Handlungsalternativen er wählen muss, dann aber die falsche Alternative innerhalb dieser Menge wählen. Oder der Fehler liegt schon in der Wahl der falschen Menge von Handlungsalternativen.

Lernen I definiert er dann als Änderung der Reaktion innerhalb derselben Menge an Handlungsalternativen.

Lernen II fasst er als Änderung im Prozess des Lernens I und definiert es als Änderung der Wahl der Menge der Handlungsalternativen, aus denen gewählt werden kann.

Lernen III fasst er als Änderung im Prozess des Lernens II und definiert es als korrigierende Veränderung im System von Handlungsalternativen, aus denen gewählt werden kann.

Lernen IV fasst er schließlich als Änderung im Lernen III und gesteht zu, dass dieses wohl nicht bei erwachsenen, lebenden Organismen auf der Erde vorkommt.

Um Batesons Lernstufenmodell zu verstehen, ist es zentral, wie die Mengen von Handlungsalternativen zustande kommen. Eine Möglichkeit, sich dies zu veranschaulichen, besteht darin, diese Mengen von Handlungsalternativen im Sinne der Sprechakttheorie von Austin (1976) und Searle (1969) zu deuten (vgl. Kosler 2016, S. 67). So weist Austin darauf hin, dass mit einem Satz wie ,Ich komme morgen wieder' unterschiedliche Handlungen vollzogen werden können. Er kann als Drohung, Versprechen oder Prognose gemeint sein. Entsprechend kann ein Gesprächspartner den Satz als Handlung richtig oder falsch verstehen. Die richtige Interpretation der Handlungsintention liefert dem Interpreten dann im Sinne Batesons eine sinnvolle Menge von Handlungsalternativen. In Batesons Modell lässt sich verstehen, wie individuelle Lernprozesse mit kollektiven Veränderungsprozessen zusammenhängen. Die Handlungskontexte, die nach Bateson jeweils mit einer Menge an sinnvollen Handlungsalternativen verbunden sind, sind keine rein 
individuellen Angelegenheiten. Dass sich eine Handlung als Drohung, als Versprechen oder auch als Prognose deuten lässt, ist ja gerade eine kollektive Vereinbarung. Zu der Frage, wie es zu neuen kollektiv ausgehandelten Mengen von Handlungsalternativen kommen kann, findet sich bei Bateson allerdings keine Antwort.

\subsection{Transformatives Lernen im Rahmen einer Sustainability Education}

Im Diskurs um Bildung für nachhaltige Entwicklung ist es insbesondere Sterling (2001, 2003, 2008, 2011, 2014) gewesen, der im Rahmen seines Konzeptes einer Sustainability Education die Idee transformativen Lernens in den Mittelpunkt gestellt hat. Dabei geht Sterling davon aus, dass sich aktuell zwei Weltbilder gegenüber stehen: Ein mechanistisches Weltbild der Moderne, das er als mechanistisch, positivistisch und dualistisch (Sterling, 2001, S. 50) charakterisiert und ein entstehendes ökologisches Weltbild im Kontext der Postmoderne (ebd., S. 49f.). Zur Erläuterung verweist er auf AutorInnen, die ausführen, die Moderne sei an einer Maschinen-Metapher orientiert, während das entstehende ökologische Weltbild an der Metapher des Organismus oder des lebenden Systems orientiert sei. Er wirft die Frage auf, inwieweit die bestehenden unterschiedlichen pädagogischen Ansätze der Erziehungswissenschaft nicht letztlich einem mechanistischen Weltbild zugeordnet werden müssen. Seinen Ansatz einer Sustainability Education ordnet er dem entstehenden ökologischen Weltbild zu. Im Mittelpunkt steht dabei die Ausbildung eines ganzheitlichen systemischen Denkens (,whole systems thinking“, ebd., S. 51), das unser Weltbild hin zu einem ökologischen Weltbild und unser pädagogisches Denken sowie unsere pädagogische Praxis verändern soll (ebd., S. 51). Um die individuelle Seite des Lernens näher zu beleuchten, unterscheidet Sterling (ebd., S. 15f.) drei Ordnungen des Lernens: Lernen erster Ordnung findet danach innerhalb akzeptierter Grenzen statt und ist adaptiv, insofern es die zugrundeliegenden Werte unhinterfragt und unverändert lässt. Lernen zweiter Ordnung sei ein kritisches, reflektiertes Lernen, das die Annahmen, die das Lernen erster Ordnung beeinflussen,

Kapelari, Suzanne (Hg.), Vierte „Tagung der Fachdidaktik“ 2019:

„Interdisziplinäre fachdidaktische Diskurse zur Bildung für nachhaltige Entwicklung“

(C) 2020 innsbruck university press, ISBN 978-3-99106-019-2, DOI 10.15203/99106-019-2 
untersucht. Lernen dritter Ordnung schließlich ermögliche es uns, Dinge anders zu sehen. Es sei kreativ, beinhaltet ein tiefes Bewusstsein von alternativen Weltbildern und Wegen, Dinge zu tun. Ein solches Lernen dritter Ordnung versteht Sterling als transformatives Lernen. Die radikale Bewegung hin zu Nachhaltigkeit erfordere individuell und gesellschaftlich ein solches transformatives Lernen.

Um die individuellen und kollektiven Transformationsprozesse genauer $\mathrm{zu}$ fassen, zieht Sterling (2003) eine Vielzahl von Theorieentwürfen heran. Von zentraler Bedeutung ist für ihn dabei der Begriff des Paradigmas, da sein Ansatz der Sustainability Education einerseits darauf zielt, zum gesellschaftlichen Wandel vom mechanistischen hin zu einem ökologischen Weltbild beizutragen, und er seinen pädagogischen Ansatz selbst als Versuch deutet, ein ökologisches Paradigma in der Pädagogik zu entwickeln, das er als Subparadigma des ökologischen Weltbildes versteht. Weltbilder werden bei ihm dabei mit Paradigmen weitestgehend gleichgesetzt (ebd., S. 120). Dazu weitet er Kuhns Modell des Paradigmenwechsels in Forschungsgemeinschaften sowohl auf Individuen als auch auf Gesellschaften aus und kombiniert es mit Batesons Stufenmodell des Lernens, um sein eigenes Modell der Ordnungen des Lernens genauer zu fassen. Das Hinterfragen eines bestehenden Paradigmas deutet er im Sinne Batesons als Lernen II: „In sum, where the whole cultural paradigm is at stage, my understanding is that Learning II is a realization of the limits of a dominant paradigm, and a move towards an alternative, even where the basis for that alternative may not be fully understood" (ebd., S. 136f.). Lernen III deutet er dann entsprechend als Beherrschung der Klasse der Paradigmen. Wer Lernen III vollzogen hat, ist danach in der Lage, zwischen verschiedenen Paradigmen zu wechseln.

Sterling (ebd.) nimmt sich auch eines Problems an, das er in seiner früheren Arbeit (2001, S. 50) nur beiläufig erwähnt. Er sieht in der Postmoderne zwar ein alternatives Weltbild, aufgrund des ihm zufolge darin enthaltenen Relativismus sei dieses aber nicht als ökologisches Weltbild geeignet. Es könne daher nur einen Zwischenschritt auf dem Weg zu einem entstehenden ökologischen 
Weltbild darstellen, das er als revisionäre Postmoderne beschreibt (Sterling 2003, S. 147f.). Dabei würde insbesondere der Dekonstruktivismus den Raum für ein ökologisches Weltbild öffnen, zugleich aber dessen Möglichkeit bestreiten. Dazu bezieht er sich insbesondere auf Lyotard (1986), der ausführt, große Erzählungen hätten in gegenwärtigen Gesellschaften einen Glaubwürdigkeitsverlust erlitten. Solche großen Erzählungen würden zum einen dazu neigen, Sprachspiele zu vereinheitlichen und die tatsächliche Vielfalt bestehender Sprachspiele auszublenden. Zudem würden sie dazu neigen, deskriptive und normative Aussagen zu vermischen (vgl. dazu Koller 2012, S. 88). Sterling unternimmt zwar nicht den Versuch eine ökologische Erzählung zu konstruieren, wie dies andere (z.B. O’Sullivan 1999) versucht haben. Aber er gibt einige Aspekte an, in denen sich ein ökologisches Weltbild vom bestehenden unterscheiden müsste (Sterling 2001, S. 170f.). Darunter eine Veränderung ,of conception of an essentially dead and inert world, to an animate, dynamic and ultimately sacred world" (ebd.). Dabei deutet sich an, dass sich in einem solchen Weltbild wieder Beschreibung und Bewertung vermischen. Angesichts des Einwandes von Lyotard bleibt damit unklar, wie im Modell von Sterling ein Übergang von einem postmodernen $\mathrm{zu}$ einem ökologischen Weltbild möglich ist.

\subsection{Transformatives Lernen in der angelsächsischen Erwachsenenbildung}

Mit der Formulierung, transformative Bildung würde auf einen Wandel individueller Bedeutungsperspektiven zielen, verweist der Fachbeirat ,Transformative Bildung/Global Citizenship Education“ auf die Diskussion um transformatives Lernen in der angelsächsischen Erwachsenenbildung. Dieser Ansatz geht auf Mezirow zurück, der ursprünglich Lernprozesse in Wiedereinstiegsprogrammen für Frauen an community-colleges, die nach einer längeren Unterbrechung ans College oder an den Arbeitsmarkt zurückkehren, untersucht hat (Mezirow 1978a, 1978b; Baumgartner 2012). Nach Mezirow erlangen die Frauen in diesen Kursen im günstigsten Fall ein kritisches Bewusstsein von den kulturellen und psychologischen Annahmen, die die

Kapelari, Suzanne (Hg.), Vierte „Tagung der Fachdidaktik“ 2019:

„Interdisziplinäre fachdidaktische Diskurse zur Bildung für nachhaltige Entwicklung“

(C) 2020 innsbruck university press, ISBN 978-3-99106-019-2, DOI 10.15203/99106-019-2 
Weise, wie wir uns selbst und unsere Beziehungen zu anderen sehen, und die Weise, wie wir unser Leben strukturieren, beeinflussen (Mezirow 1978b, S. 101). Genau dies bezeichnet er als Lernen über Bedeutungsperspektiven (ebd.). Er führt explizit in Analogie zu Kuhns Theorie vom Wechsel wissenschaftlicher Paradigmen aus: „A meaning perspective refers to the structure of cultural assumptions within which new experience is assimilated to - and transformed by - one's past experience. It is a personal paradigm for understanding ourselves and our relationships“ (ebd.). Als mögliche Auslöser für solche Lernprozesse identifiziert er Herausforderungen und Dilemmata des Erwachsenenlebens. Das Ziel eines solchen Prozesses ist für Mezirow 1978 noch ein kohärentes, autonomes Subjekt. Der Prozess wird im Wesentlichen als Rollenwechsel verstanden (ebd.; vgl. Kosler, 2016, S. 71ff.). Zur weiteren Ausgestaltung seines Ansatzes zieht Mezirow ab 1981 dann die Unterscheidung von drei Erkenntnisinteressen, die Habermas (1968) in seiner Frankfurter Antrittsvorlesung getroffen hat, heran (Mezirow 1981, S. 3ff.; vgl. Kosler, 2016, S. 73f.). Den Prozess der Perspektiventransformation deutet er nun in Anlehnung an Habermas als „emancipatoric process of becoming critical aware of how and why the structure of psycho-cultural assumptions come to constrain the way we see ourselves and our relationships, reconstituting this structure to permit a more inclusive and discriminating integration of experience and acting upon these new understandings" (Mezirow, 1981, S. 6). Später beschreibt Mezirow das Werk von Habermas als soziolinguistischen Rahmen seiner Transformationstheorie (Mezirow, 1997, S. 143). Bedeutungsperspektiven sind bei Mezirow nun wiederum aus Bedeutungsschemata zusammengesetzt: „Ein Bedeutungsschema stellt ein bestimmtes Wissen, bestimmte Überzeugungen, Werturteile und Empfindungen dar, die in einer Interpretation ihren Ausdruck finden. Bedeutungsschemata sind die konkreten Manifestationen unserer gewohnheitsmäßigen Orientierung und Erwartungen (Bedeutungsperspektiven) und übertragen diese allgemeinen in besondere Erwartungen, die uns bei unseren Handlungen leiten“ (ebd., S. 36). Als Beispiel gibt er den Ethnozentrismus als Mittelpunkt einer Bedeutungsperspektive an und bestimmt dann rassische und sexuelle Klischees als Bedeutungsschemata innerhalb dieser Perspektive. Auf Basis der oben erläuterten Lerntheorie von Bateson, die er als

Kapelari, Suzanne (Hg.), Vierte „Tagung der Fachdidaktik“ 2019:

„Interdisziplinäre fachdidaktische Diskurse zur Bildung für nachhaltige Entwicklung“

(C) 2020 innsbruck university press, ISBN 978-3-99106-019-2, DOI 10.15203/99106-019-2 
„bahnbrechende Analyse“ charakterisiert (ebd., S. 77), beschreibt er vier Formen des Lernens in der Erwachsenenbildung. Er reformuliert dazu Batesons Stufenmodell mit Hilfe seiner Begriffe des Bedeutungsschemas und der Bedeutungsperspektive. Lernen II ist danach ein Lernen, das Bedeutungsschemata verändert, während Lernen III die Veränderung der Bedeutungsperspektive beinhaltet. Damit unterscheidet Mezirow die folgenden vier Formen des Lernens (ebd., S. 77f.):

1. Lernen durch Bedeutungsschemata, als Differenzierung und Verfeinerung der als gegeben angesehenen Bedeutungsschemata oder durch Lernen innerhalb der Struktur eines Bezugsrahmens.

2. Erlernen neuer Bedeutungsschemata, als Schaffung neuer Bedeutungen, die mit bestehenden Bedeutungsperspektiven vereinbar sind, insofern diese dadurch in ihrem Anwendungsbereich erweitert werden.

3. Lernen durch Transformation von Bedeutungsschemata, die als dysfunktional erkannt wurden.

4. Lernen durch Transformation ganzer Bedeutungsperspektiven.

Damit hat Mezirow ein differenziertes Raster vorgelegt, in dem verschiedene Formen des Lernens unterschieden werden können. Durch die Adaption der Lerntheorie von Bateson liefert Mezirows Ansatz eine analoge Antwort auf die Frage, wie individuelles Lernen mit kollektiven Bewusstwerdungs- und Emanzipationsprozessen zusammenhängt. Die Bedeutungsperspektiven bei Mezirow sind ebenfalls keine rein individuelle Angelegenheit. Bedeutungsperspektiven sind auch bei ihm kollektiv entwickelte Möglichkeiten, unseren Strom an Eindrücken und Erfahrungen zu strukturieren und mit Sinn zu versehen. Offen bleibt bei Mezirow allerdings, wie individuelle und kollektive Veränderungsprozesse ineinandergreifen, um neue Bedeutungsperspektiven hervorzubringen. Genau dies wäre im Hinblick auf das Verhältnis individueller und gesellschaftlicher Transformationsprozesse im Kontext einer nachhaltigen Entwicklung relevant. Dass diese Frage bei Mezirow nicht auftritt, liegt vermutlich daran, dass sein Ansatz daran orientiert ist, wie individuelle Transformationsprozesse im Kontext der feministischen Bewegung unterstützt werden können. Dabei scheint mir eine theoretische Neubestimmung der Rollenverteilung von Frauen und Männern in heterosexuellen Paarbeziehungen

Kapelari, Suzanne (Hg.), Vierte „Tagung der Fachdidaktik“ 2019:

„Interdisziplinäre fachdidaktische Diskurse zur Bildung für nachhaltige Entwicklung“

(C) 2020 innsbruck university press, ISBN 978-3-99106-019-2, DOI 10.15203/99106-019-2 
bereits Teil des Rahmens gewesen zu sein, der durch die Politisierung dieser Thematik durch den Feminismus im Bewusstsein großer Teile der Bevölkerung angekommen war. Das Ziel der von Mezirow beschriebenen Seminare bestand dann darin, Menschen, die für sich bereits beschlossen hatten, aufgrund solcher Einsichten ihr Leben zu ändern, dabei zu unterstützen. Vermutlich sind manche Menschen auch erst in solchen Seminaren $\mathrm{zu}$ entsprechenden Einsichten gekommen. Aber die eigentliche Aufgabe bestand darin, aufgrund solcher Einsichten, das eigene Fühlen und Handeln, insbesondere eingeübte Verhaltensroutinen, zu ändern. Dabei sind neue Handlungsweisen relativ einfach aus der theoretischen Analyse abzuleiten gewesen, z.B. eben nicht mehr jeden Abend dem Ehemann das Essen zuzubereiten. Die Ausgangslage scheint mir im Kontext einer nachhaltigen Entwicklung eine andere zu sein. Neue Bedeutungsperspektiven müssen ja erst entwickelt werden und es stellt sich die Frage, wie transformatives Lernen dazu beitragen kann.

\subsection{Der transformatorische Bildungbegriff der deutschsprachigen Bildungstheorie}

Aus meiner Sicht wäre der in der deutschsprachigen Bildungstheorie insbesondere von Kokemohr (1989, 1992, 2007), Marotzki (1990) und Koller (2012) entwickelte Begriff transformatorischer Bildung als Ansatzpunkt besser geeignet, um im Kontext einer nachhaltigen Entwicklung genauer zu fassen, wie individuelle und gesellschaftliche Transformationsprozesse aufeinander bezogen sind. Ausgangspunkt dieser Konzeption ist zunächst eine Unterscheidung von Lernen und Bildung, derzufolge Lernen als Prozess der Aufnahme, Aneignung und Verarbeitung neuer Informationen zu verstehen ist, während Bildung als Lernprozess höherer Ordnung $\mathrm{zu}$ verstehen ist, bei dem nicht nur neue Informationen angeeignet werden, sondern auch der Modus der Informationsverarbeitung sich grundlegend ändert (Koller, 2012, S. 15). Diese Unterscheidung wurde von Matrotzki (1990) und Kokemohr (1992) auch explizit als bildungstheoretischer Kern des Batesonschen Stufenmodells ausgewiesen. Die Grundidee dieses Ansatzes besteht in dem Vorschlag, Bildung

Kapelari, Suzanne (Hg.), Vierte „Tagung der Fachdidaktik“ 2019:

„Interdisziplinäre fachdidaktische Diskurse zur Bildung für nachhaltige Entwicklung“

(C) 2020 innsbruck university press, ISBN 978-3-99106-019-2, DOI 10.15203/99106-019-2 
als „Prozess der Be- oder Verarbeitung solcher Erfahrungen zu untersuchen, die der Subsumption unter Figuren eines gegebenen Welt- und Selbstentwurfs widerstehen“ (Kokemohr, 2007, 21). Kokemohr sieht es dann als gerechtfertigt an, von Bildung zu sprechen, „wenn der Prozess der Be- oder Verarbeitung subsumptionsresistenter Erfahrungen eine Veränderung von Grund legenden Figuren meines je gegebenen Welt- und Selbstentwurfs einschließt“ (ebd.). Koller hebt zwei Elemente besonders hervor, die aus seiner Sicht neu an Kokemohrs Vorschlag gegenüber Humboldts Bildungsbegriff sind. Zum einen bestimmt Kokemohr den Anlass für einen solchen Bildungsprozess als Krisenerfahrung, die in der Konfrontation mit einer Problemlage besteht, für deren Bewältigung sich das bisherige Welt- und Selbstverhältnis als nicht ausreichend erweist (Koller, 2012, S. 16). Zum anderen hebt er den Anspruch Kokemohrs hervor, seinen Bildungsbegriff als anschlussfähig für qualitativempirische Untersuchungen zu fassen (ebd., S. 16f.). Bildung mit Kokemohr und Koller als Transformation von Figuren des Welt- und Selbstverhältnisses von Menschen angesichts neuer Problemlagen, für deren Bewältigung die Figuren ihres bisherigen Welt- und Selbstverhältnisses nicht mehr ausreichen, zu fassen, kann dabei nicht als Definition, sondern eher als Vorschlag für die Form einer Definition verstanden werden. So benennt Koller vier Dimensionen, in denen eine nähere Bestimmung nötig ist (ebd., S. 17), die sich als Fragen formulieren lassen:

1. Wie lassen sich die Strukturen der Selbst- und Weltverhältnisse beschreiben?

2. Welche Problemlagen oder Krisenerfahrungen können transformatorische Bildungsprozesse auslösen? Und welche tun dies typischerweise?

3. Wie verlaufen solche Prozesse und welche Prozessstrukturen lassen sich dabei identifizieren? Wie kann Neues in einem solchen Prozess entstehen?

4. Auf welche Weise ist ein empirischer Zugang $\mathrm{zu}$ transformatorischen Bildungsprozessen möglich?

Im Kontext nachhaltiger Entwicklung stellt sich darüber hinaus die Frage, wie ein individueller Bildungsprozess als Beitrag $\mathrm{zu}$ einer gesellschaftlichen Transformation verstanden werden kann. Diese Frage stellt Koller selbst zwar nicht, aber es lassen sich mit ihm zumindest Ansatzpunkte für eine Antwort 
finden. So schließt Koller (ebd., S. 88ff.) an Lyotards „Der Widerstreit“ (1989) an, in dem dieser sich mit der Legitimation gesellschaftlicher Ordnung befasst. In der Interpretation Kollers, der ich hier folge, geht Lyotard nicht davon aus, dass Subjekte vor oder unabhängig von der Äußerung von Sätzen bestehen, sondern davon, dass sie in Sätzen als Sender, Empfänger oder Referenten situiert werden. Obwohl jeder Satz als eigenes Universum betrachtet wird, lassen sich Darstellungsarten von Universen und damit Satzfamilien unterscheiden, die verknüpft sind mit Regeln der Satzbildung. Entsprechend lassen sich beispielsweise deskriptive, präskriptive, evaluative, interrogative oder ostensive Sätze unterscheiden. Entscheidend ist nun, dass „Sätze unterschiedlicher Regelsysteme [...] nicht ineinander übersetzt werden“ (Lyotard, 1989, S. 92) können. Sätze können allerdings verkettet werden und solche Verkettungen konstituieren Diskursarten, die Lyotard als Regelsysteme höherer Ordnung betrachtet, die die möglichen Verkettungen unter einen bestimmten Zweck stellt. Als Beispiele zitiert Koller bei Lyotard unter anderem „Wissen, Lehren, Rechthaben, Verführen, Rechtfertigen, Bewerten, Erschüttern, Kontrollieren“ (Koller, 2012, S. 91). Solche Zwecke dürfen dabei nicht als Intentionen von Subjekten, sondern im Sinne von Strategien, die Spielern „auferlegt" (ebd.) werden, verstanden werden. Entsprechend ließen sich schließlich verschiedene Diskursarten wie wissenschaftliche, ökonomische, philosophische und narrative unterscheiden. Der besondere Clou besteht nun darin, dass es beim „Aufeinandertreffen von unterschiedlichen Diskursarten unvermeidlich zu einem Widerstreit (différend) kommt, d.h. zu einem Konflikt, der prinzipiell nicht zu schlichten ist, weil eine übergreifende Urteilsregel fehlt" (ebd., S. 92). Genau hierin besteht die Idee einer radikalen Heterogenität der Diskursarten.

Mit einer solchen Konzeption kann nun Koller im Hinblick auf die ersten beiden der erwähnten vier näheren Bestimmungen des Bildungsbegriffes zumindest Ansatzpunkte beschreiben. Zum einen lässt sich mit Lyotard verstehen, dass die Struktur von Welt- und Selbstverhältnissen als Resultat der Verkettung von Sätzen nach diskursiven Regeln $\mathrm{zu}$ fassen sei und die Positionierung von Subjekten im Verhältnis zur Welt und zu sich selbst damit 
nicht als Ausdruck einer der Sprache vorgängigen Subjektivität zu verstehen ist. Als Problemlagen, die Bildungsprozesse auslösen können, sind so Fälle von Widerstreit zu betrachten. Koller kann aus dem Rekurs auf Lyotard auch eine Minimalethik seiner Bildungstheorie ableiten: Diese besteht in der Anerkennung der radikalen Heterogenität der Diskursarten, aus der er die Forderung ableitet, ein bereits artikulierter Widerstreit müsse offen gehalten werden, um zu verhindern, dass durch Heranziehen einer vermeintlich übergeordneten Regel eine Seite der Möglichkeit, sich zu artikulieren, beraubt würde. Außerdem müsse auch dort, wo kein offener Konflikt zu erkennen sei, „,sondern ein Schweigen oder ein ,Gefühl' anzeigt, dass in der vorherrschenden Diskursart ,etwas“ nicht gesagt werden kann“ (ebd., 94), mit der Möglichkeit des Widerstreits gerechnet werden. Damit versteht Koller Bildung ,als jenen Prozess der Entstehung neuer Sätze und Diskursarten [...], der zur Anerkennung und zum Offenhalten des Widerstreits erforderlich ist“" (ebd., S. 97). So ist klar, dass durch die individuelle Artikulation neuer Sätze und die neuartige Verkettung von Sätzen neue Diskursarten geschaffen werden können.

Im Hinblick auf die Frage, wie individuelles Lernen und die Transformation kollektiver Denkstrukturen zusammenhängen, liefert Koller mit seiner Interpretation Lyotards eine völlig neue Perspektive. Subjekte denkt Lyotard gar nicht als unabhängig von Sätzen und Diskursarten. Subjekte situieren sich erst in der Äußerung von Sätzen und diese sind durch kollektiv ausgehandelte Regelsysteme, die Diskursarten, reguliert. Wenn ein individueller Bildungsprozess über die Artikulation neuer Sätze neue Diskursarten hervorbringt, dann ist Bildung bei Koller so immer ein Akt der Veränderung von Gesellschaft. Was bei Lyotard fehlt, sind allerdings Hinweise darauf, wie die Hervorbringung neuer Diskursarten $\mathrm{zu}$ bewerkstelligen ist. Lyotards Analyse weist bloß darauf hin, dass dies möglich sei.

Für die Beantwortung der Frage, wie im individuellen Bildungsprozess Neues entstehen kann, diskutiert Koller sieben verschiedene Theorieansätze. Darunter auch Kuhns Paradigmenwechsel in der Naturwissenschaft, da Kuhn selbst von einem plötzlichen Umschwung in seinem Denken über die aristotelische Physik

Kapelari, Suzanne (Hg.), Vierte „Tagung der Fachdidaktik“ 2019:

„Interdisziplinäre fachdidaktische Diskurse zur Bildung für nachhaltige Entwicklung“

(C) 2020 innsbruck university press, ISBN 978-3-99106-019-2, DOI 10.15203/99106-019-2 
ausgegangen war und dann nach dem Vorbild dieses individuellen Prozesses seine Theorie eines kollektiven Paradigmenwechsels formuliert hatte. Im Hinblick auf den Zusammenhang von individuellem Lernen und der Transformation kollektiver Denkstrukturen ist dabei insbesondere der von Koller herangezogene Ansatz von Derrida relevant. Dazu schließt Koller an Derridas Aufsatz „Signatur Ereignis Kontext“ (1988) an. Ich fasse dazu Kollers Interpretation zusammen. Während im gewöhnlichen Verständnis Kommunikation als Übermittlung eines Sinns aufgefasst wird (Derrida, 1988, S. 291; Koller, 2012, S. 127), zeigt Derrida, dass dies nur möglich ist, wenn der jeweilige Kontext begrenzt werden kann. Derridas These besteht nun darin, dass ein solcher Kontext nie völlig bestimmt und eingegrenzt werden kann. Dabei geht Derrida von einer bestimmten Form der Kommunikation, nämlich der Schrift aus. Nach dem gängigen Verständnis fassen wir die Schrift als ein Mittel zur Übermittlung von Sinn auf, welches es uns beispielsweise erlaubt, mit Personen zu kommunizieren, die sich nicht zur selben Zeit am selben Ort wie wir befinden. Derrida hebt nun hervor, dass die Schrift vom Empfänger und vom Sender abgelöst ist und dass die sie ausmachenden Schriftzeichen iterierbar, also wiederholbar, sind. Außerdem ist sie von den Intentionen und vom Bewusstsein des Senders losgelöst und damit dessen Kontrolle entzogen. Die Schrift ist abgelöst vom „Horizont des Sinns“ (Derrida, 1988, S. 299; Koller, 2012, S. 128), den Koller als Vorstellung eines geschlossenen und eindeutig feststellbaren Sinns interpretiert, und vom Kontext, wie sich am Beispiel des Zitats sehen lässt, in dem ein neuer Kontext aufgepfropft wird. Entsprechendes gilt für gesprochene Sprache, da auch sie nur funktionieren kann, wenn sie unabhängig von demjenigen, der etwas geäußert hat, und vom Kontext, in dem dies geschah, iterierbar ist. Diese Ablösbarkeit könne man beispielsweise an widersinnigen Äußerungen wie „der Kreis ist viereckig“ oder „das grün ist oder“" sehen. Und schließlich erläutert Koller, dass Derrida die Möglichkeit des Misslingens performativer Sprechakte, anders als Austin, nicht als Anomalie deutet. Ein Beispiel wäre die feierliche Äußerung eines Schauspielers im Rahmen eines Theaterstückes „Ich taufe dieses Schiff auf den Namen Anna“, mit dem das dem Schauspieler gegenüberstehende Objekt eben nicht getauft wird. Derrida vertritt im Gegenteil die Auffassung, das Gelingen

Kapelari, Suzanne (Hg.), Vierte „Tagung der Fachdidaktik“ 2019:

„Interdisziplinäre fachdidaktische Diskurse zur Bildung für nachhaltige Entwicklung“

(C) 2020 innsbruck university press, ISBN 978-3-99106-019-2, DOI 10.15203/99106-019-2 
performativer Sprechakte sei gerade an die Ausnahme gebunden, dass die Intention des sprechenden Subjektes anwesend ist. Daraus folgert Derrida, dass Kommunikation nicht als Übermittlung von Sinn verstanden werden dürfe und auch nicht Gegenstand eines hermeneutischen Verstehens sein könne, das auf die „Entzifferung eines Sinns oder einer Wahrheit“ (Derrida, 1988, S. 299; Koller, 2012, S. 129) ausgerichtet ist. Alternativ schlägt Derrida dann eine Lektüre vor, die der Zerstreuung und Vervielfältigung des Sinns folgt. Damit sei Derridas Konzept einer dekonstruktiven Lektüre zumindest angedeutet. Neue Lesarten würden durch den „Nachvollzug der potentiellen und nie völlig beherrschbaren Vieldeutigkeit eines Textes bzw. - übertragen auf Bildungsprozesse - einer Erfahrung“ (Koller, 2012, S. 129) entstehen. Koller betont dabei, dass damit, wie schon im hermeneutischen Ansatz, die Entstehung des Neuen als Prozess unablässiger Auseinandersetzung mit dem Gegenstand verstanden wird und insofern Derrida am Vorrang der Sache und der Notwendigkeit einer Infragestellung der eigenen Position der Leserin bzw. des Lesers festhalte (ebd.).

\subsection{Bildungstheoretische Schlussfolgerungen für den naturwissenschaft lichen Unterricht}

Kollers transformatorischer Bildungsbegriff eignet sich, um einen Zusammenhang zwischen einem individuellen Bildungsprozess und einem gesellschaftlichen Transformationsprozess herzustellen. Mit Lyotard ist ein transformatorischer Bildungsprozess als Hervorbringung neuer Sätze und Diskursarten zu verstehen, die einen Widerstreit zu bestehenden Diskursarten und Sätzen erzeugt oder einen solchen aufrecht erhält. Mit Derrida ist die dekonstruktive Lektüre eines Textes, die Koller auch auf Erfahrungen erweitert, eine Möglichkeit, um aus der nach Derrida bestehenden potentiellen Vieldeutigkeit neuen Sinn hervorzubringen. Für naturwissenschaftliches Denken bedeutet dies, dass aus einer dekonstruktiven Lektüre der Naturwissenschaft ein neuer Diskurs erzeugt werden kann, der im Widerstreit zum naturwissenschaftlichen Diskurs steht. Eine solche Dekonstruktion der

Kapelari, Suzanne (Hg.), Vierte „Tagung der Fachdidaktik“ 2019:

„Interdisziplinäre fachdidaktische Diskurse zur Bildung für nachhaltige Entwicklung“

(C) 2020 innsbruck university press, ISBN 978-3-99106-019-2, DOI 10.15203/99106-019-2 
Naturwissenschaft sehe ich dabei zunächst nicht als sinnvolles Ziel innerhalb naturwissenschaftlichen Unterrichts. Eine Dekonstruktion der Naturwissenschaft ist ein Weg, um einen widerstreitenden Diskurs über die Natur hervorzubringen und aus meiner Sicht zunächst Aufgabe der Philosophie. Fordert man als Elemente einer Bildung für eine nachhaltige Entwicklung eine transformative Bildung, so wird die Dekonstruktion des naturwissenschaftlichen Denkens auch zu einer Aufgabe für die naturwissenschaftlichen Didaktiken. Ein angemesseneres Ziel für eine an einer nachhaltigen Entwicklung orientierten naturwissenschaftlichen Bildung bestünde aus meiner Sicht darin, SchülerInnen sowohl an den naturwissenschaftlichen Diskurs als auch an einen widerstreitenden Diskurs über Natur heranzuführen. Als Offenhalten eines Widerstreites, der aufgrund der Dominanz des naturwissenschaftlichen Diskurses immer in der Gefahr steht, unartikulierbar zu werden, wäre die Aneignung eines solchen Diskurses selbst als Bildungsprozess im Sinne Kollers zu deuten.

\section{Transformative Bildung im naturwissenschaftlichen Unterricht}

Es stellt sich die Frage, wie sich gegenüber der naturwissenschaftlichen Betrachtung der Natur ein widerstreitender Diskurs bestimmen lässt, der einen alternativen Zugang zur Natur bietet. Eine Möglichkeit dafür bietet der französische Philosoph und Sinologe Jullien (1999, 2004, 2010), der die Grundannahmen des europäischen mit dem chinesischen Denken vergleicht. Ein Vergleich der Grundannahmen europäischen Denkens mit dem chinesischen Denken ist für eine Bestimmung der Eigenheiten naturwissenschaftlichen Denkens vielversprechend: Die neuzeitliche Naturwissenschaft hat sich nur in Europa entwickelt und nicht anderswo. China ist zudem die einzige große Kultur, die, weitestgehend ohne Austausch mit Europa und auch ohne Verwandtschaft der Sprachen, bis zum Beginn des Austausches in der zweiten Hälfte des 16. Jahrhunderts ähnlich entwickelt ist im Bereich der Technik

Kapelari, Suzanne (Hg.), Vierte „Tagung der Fachdidaktik“ 2019:

„Interdisziplinäre fachdidaktische Diskurse zur Bildung für nachhaltige Entwicklung“

(C) 2020 innsbruck university press, ISBN 978-3-99106-019-2, DOI 10.15203/99106-019-2 
(Schiffbau) und gesellschaftlicher Institutionen (Polizei) (Jullien, 2006, S. 7). China ist insofern für Europa die ,fremdeste Kultur“ (Jullien, 2008a, S. 8) und eignet sich damit besonders gut als Kontrastfolie, vor der die unhinterfragten Grundannahmen des europäischen Denkens sichtbar gemacht werden können. Entsprechend bezeichnet Jullien sein eigenes Vorgehen auch als „Dekonstruktion von außen“" (ebd., S. 10). Jullien geht methodisch innerhalb der Sinologie dabei einen Mittelweg. Er geht weder davon aus, dass das chinesische Denken dem europäischen so fremd ist, dass es ohnehin nicht verstanden werden kann, noch davon, dass beide Denkweisen einen gemeinsamen Gegenstand haben (Jullien, 2008b, S. 89f.). Er beschreibt sein methodisches Vorgehen so, dass er im Vergleich der Denktraditionen immer zunächst nach einer beiden Denkweisen gemeinsamen Frage sucht: „Im Rahmen der Frage kann [...] die Spaltung zwischen zwei möglichen Optionen beginnen, die sich nach und nach aus ihr ergeben und allmählich die Alternative bilden, aus der eine Vorstellung der Andersartigkeit hervorgeht" (ebd., S. 94).

Unter den von Jullien herausgearbeiteten Grundannahmen europäischen Denkens lassen sich zwei identifizieren, die in der Naturwissenschaft entfaltet wurden: die Orientierung des Denkens am Modell nach dem Vorbild der euklidischen Geometrie und die Idee, Veränderung in der Natur nach dem Vorbild der Bewegung eines Körpers von einem Anfangspunkt bis zu einem Endpunkt zu betrachten. Beide Grundannahmen wurden unter Rekurs auf die jüngere Wissenschaftsforschung näher untersucht und expliziert (vgl. Kosler, 2016).

Es stellt sich also zunächst die Frage, worin die Orientierung des Denkens am Modell nach dem Vorbild der euklidischen Geometrie besteht. Unter Rekurs auf Netz (1999) konnte gezeigt werden (Kosler, 2016), dass das charakteristische an der euklidischen Geometrie in der Verwendung von Diagrammen besteht. Die Verwendung dieser Diagramme bringt zwei besondere Vorteile mit sich. Zum einen reduziert die Nutzung der Diagramme den Gegenstandsbereich, über den im Zuge der Beweisführung gesprochen wird. Dies geschieht dadurch, dass in den Diagrammen jeweils wenige Punkte durch Buchstaben bezeichnet werden.

Kapelari, Suzanne (Hg.), Vierte „Tagung der Fachdidaktik“ 2019:

„Interdisziplinäre fachdidaktische Diskurse zur Bildung für nachhaltige Entwicklung“

(C) 2020 innsbruck university press, ISBN 978-3-99106-019-2, DOI 10.15203/99106-019-2 
In der Beweisführung wird nur auf eine kleine Anzahl geometrischer Objekte wie Punkte, Linien oder Flächen Bezug genommen. Sie alle können über die ins Diagramm eingezeichneten Buchstaben bezeichnet werden. Zum zweiten steht das jeweilige Diagramm für eine beliebig große Zahl ähnlicher geometrischer Objekte, die mit dem im Diagramm repräsentierten Objekt diejenigen Eigenschaften teilen, die im Rahmen der Beweisführung angenommen werden. Dies ermöglicht es am Ende der Beweisführung der Leserin und dem Leser, sich davon zu überzeugen, dass der am jeweiligen Diagramm geführte Beweis unverändert auch für all diese geometrischen Objekte, für die das Diagramm als Stellvertreterobjekt fungiert, geführt werden könnte, und so der Beweis allgemeingültig ist. Die Verwendung der Diagramme ermöglicht es damit, den Gegenstandsbereich für die Zwecke der Beweisführung zunächst auf sehr wenige Objekte $\mathrm{zu}$ reduzieren und dennoch am Ende allgemeingültige Aussagen $\mathrm{zu}$ erhalten. Jullien zufolge entfaltete sich die Orientierung des Denkens am Modell nach dem Vorbild der Geometrie in der neuzeitlichen Naturwissenschaft, indem Galilei einen Weg fand, Naturwissenschaft nach diesem Vorbild zu betreiben.

Die Idee, Veränderung in der Natur nach dem Vorbild der Bewegung eines Körpers zu betrachten, lokalisiert Jullien bereits in der Physik des Aristoteles. Unter Heranziehung einschlägiger Arbeiten der jüngeren Wissenschaftsphilosophie und -geschichte wurde untersucht, wie Galilei diese beiden Grundannahmen des Denkens aufnahm. Es zeigte sich, dass Galilei gerade den Aristotelischen Begriff der Veränderung am Beispiel der Bewegung eines Körpers weiterentwickeln musste, um die Arbeit mit Diagrammen aus der euklidischen Geometrie in die Naturwissenschaft überführen zu können. Nach Aristoteles lassen sich Veränderungen stets durch die Angabe eines Anfangszustandes, eines Endzustandes und des Objektes, an dem sich die Veränderung vollzieht, charakterisieren (Ackrill, 1985). Entsprechend lässt sich das Herunterfallen eines Körpers durch Angabe des Ortes, an dem der Körper losgelassen wird, des Ortes, an dem der Körper am Boden ankommt, und des Körpers selbst charakterisieren. Galilei weicht nun von diesem Veränderungsverständnis $a b$, indem er den Begriff des Geschwindigkeits- 
momentes einführt. Damit lässt sich das Fallen eines Körpers auch in einem Moment charakterisieren. Es ist damit nicht mehr notwendig, einen Anfangsund einen Endzustand anzugeben. Damit lassen sich nun Veränderungen nicht mehr nur als endliche Bewegungen auffassen, sondern als Bewegungszustände.

Dieser Begriff des Geschwindigkeitsmomentes erlaubt es Galilei, ein euklidisches Diagramm zu zeichnen, um das Fallen eines Körpers zu repräsentieren (Galilei, 2007, S. 158f., vgl. Kosler, 2016, S. 224ff.). Dazu zeichnet Galilei eine senkrechte Linie, die die Zeit repräsentiert, die der Körper fällt. Senkrecht dazu zeichnet Galilei Linien ein, die das Geschwindigkeitsmoment des fallenden Körpers zum jeweiligen Zeitpunkt repräsentieren. Zusätzlich zeichnet Galilei dann noch eine Bewegung mit konstanter Geschwindigkeit ins Diagramm ein und kann so Beziehungen zwischen diesen beiden Bewegungen herstellen und sein zweites Fallgesetz ableiten. Ein Grundproblem bei der Verwendung von Diagrammen in der Naturwissenschaft bestand zunächst darin, dass Naturwissenschaft seit Aristoteles als Analyse der Bewegung von Körpern aufgefasst wurde, sich in den Diagrammen der euklidischen Geometrie jedoch nichts bewegt. Galileis Trick besteht insofern also darin, mit der Zeit und dem Geschwindigkeitsmoment Begriffe heranzuziehen, mit denen sich Bewegung als Aufeinanderfolge von Zuständen beschreiben lässt und dann Diagramme zu finden, deren Elemente einzelne Bewegungszustände repräsentieren.

Naturwissenschaftliches Denken lässt sich nach dieser Analyse als ein Denken charakterisieren, in dem Naturveränderungen nach dem Vorbild der Bewegung von Körpern betrachtet werden und Veränderungsbegriffe verwendet werden, die es erlauben, Veränderungen im Moment und damit auch als Zustand aufzufassen. Damit wird es möglich, endliche Bewegungen als Abfolge von Zuständen aufzufassen und solche Zustände innerhalb einer Bewegung oder zwischen verschiedenen Bewegungen mit Hilfe von Diagrammen zueinander ins Verhältnis $\mathrm{zu}$ setzen und so unbekannte Verhältnisse aus bekannten abzuleiten. Dies ermöglicht es, Naturgesetze aufgrund von plausiblen Annahmen argumentativ herzuleiten. 
Da mit Hilfe von Naturgesetzen Vorhersagen in die Zukunft getroffen werden können und ebensolche Vorhersagen notwendig sind, um Entscheidungen zu treffen, lässt sich nun auch näher beschreiben, in welchem Verhältnis naturwissenschaftliches Denken zur Gestaltung einer nachhaltigen Entwicklung steht. Naturwissenschaftliches Denken ist insofern nützlich für die Gestaltung einer nachhaltigen Entwicklung, als es Vorhersagen in die Zukunft ermöglicht. Und es ist insofern hinderlich für die Gestaltung einer nachhaltigen Entwicklung, als es von einzelnen Objekten und partikularen Zusammenhängen ausgeht und so der Gesamtzusammenhang aller Dinge und Prozesse zunächst ignoriert wird. Diese Analyse ist dabei nicht neu. Neu ist, dass sich mit der zuvor vorgeführten Charakterisierung erklären lässt, warum dies der Fall ist: Es ist die spezifische Verwendung von Diagrammen, die allgemeingültige Argumente und damit die Herleitung von Gesetzen möglich macht und so Aussagen über die Zukunft erlaubt. Und es ist die Verwendung eben dieser Diagramme, die den Gegenstandsbereich des Nachdenkens auf wenige Objekte und Zusammenhänge beschränkt.

Für die Naturwissenschaftsdidaktik stellt sich die Frage, wie es möglich ist, mit SchülerInnen die Vor- und Nachteile naturwissenschaftlichen Denkens auch zu reflektieren. Diese Frage drängt sich angesichts der Abstraktheit meiner Ausführungen zum Wesen naturwissenschaftlichen Denkens besonders auf. Aus meiner Sicht und im Anschluss an Jullien kann das, wie schon die Analyse selbst, in Form einer Kontrastierung passieren. Dazu müsste man Beispiele nach dem Vorbild der Bewegung von Körpern auswählen, SchülerInnen dazu anregen, mit Skizzen zu argumentieren, Veränderungsraten einführen und das eigene Denken immer wieder durch Versuche überprüfen. Dass das Argumentieren mit Skizzen schon in der Primarstufe prinzipiell möglich ist, haben Lehrer und Schauble von der Vanderbilt University in den letzten 20 Jahren immer wieder mit Klassen der Jahrgangsstufen zwei, drei und vier exemplarisch vorgeführt (Lehrer et al. 2000; Lehrer \& Schauble, 2012). Neu zu konzipieren wäre eine Kontrastierung mit einem chinesischen Herangehen für das Nachdenken über Naturveränderungen. Charakteristisch für das traditionelle chinesische Denken ist, dass der Gegenstand des Nachdenkens ganzheitliche 
stille Wandlungsprozesse sind (vgl. Kosler 2016). Das paradigmatische Beispiel dafür sind die Jahreszeiten. Damit sind Veränderungen nicht als Veränderung einer Eigenschaft eines Objektes gefasst, sondern als Veränderung an einer Gesamtheit, die sich an vielen einzelnen Veränderungen manifestiert. Außerdem treten typische Veränderungen zyklisch immer wieder auf. Die Veränderung der Jahreszeiten ist also nicht etwas, das einen definierten Anfangs- und Endzustand hat, sondern etwas, das ohne Anfang und Ende und damit endlos ist. Für die Beschreibung werden zwei miteinander zusammenhängende Faktoren postuliert, die abwechselnd in den Vordergrund und in den Hintergrund treten und sich in den vielen einzelnen Veränderungen manifestieren. Im Falle der Jahreszeiten sind dies die Wärme des Sommers und die Kälte des Winters, die einander abwechseln und die die betrachtete Gesamtheit jeweils auf spezifische Weise umwandeln. Veränderung wird dabei nicht im Sinne einer zeitlichen Ordnung eines Nacheinanders von Zuständen gedacht, sondern ein zeitlicher Aspekt kommt dadurch ins Spiel, dass jeder Moment der Veränderung so verstanden wird, dass es etwas gibt, das geht, und etwas, das kommt. Das Ziel eines solchen Denkens besteht nicht darin, Zusammenhänge zwischen einzelnen Aspekten zu finden und Vorhersagen zu machen, sondern darin, einen solchen Wandlungsprozess wahrzunehmen, die ihn bestimmenden Faktoren zu erfassen und Gelegenheiten aufzuspüren, in denen der Prozess durch geeignete Maßnahmen so beeinflusst werden kann, dass er sich von selbst in eine gewünschte Richtung entwickelt.

\section{Literatur}

Ackrill, J. L. (1985). Aristoteles. Berlin: Gruyter.

Austin, J. L. (1976). How to do Things with Words. Oxford: Oxford University Press.

Bateson, G. (1987). The logical categories of learning and communication. In G. Bateson, Steps to an Ecology of Mind. Collected Essays in Anthropology, Psychiatry, Evolution, and Epistemology (S. 279-308). Northvale: Aronson.

Baumgartner, L. M. (2012). Mezirow's theory of transformative learning from 1975 to present. In E. W. Taylor, P. Cranton and Associates (Ed.), The Handbook of

Kapelari, Suzanne (Hg.), Vierte „Tagung der Fachdidaktik“ 2019:

„Interdisziplinäre fachdidaktische Diskurse zur Bildung für nachhaltige Entwicklung“

(C) 2020 innsbruck university press, ISBN 978-3-99106-019-2, DOI 10.15203/99106-019-2 
Transformative Learning. Theory, Research, and Practice (S. 99-115). San Francisco: Jossey-Bass.

Derrida, J. (1988). Signatur Ereignis Kontext, In J. Derrida, Randgänge der Philosophie (S. 291-314). Wien: Passagen.

Galilei, G. (2007 [1638]). Unterredungen und mathematische Demonstrationen über zwei neue Wissenszweige, die Mechanik und die Fallgesetze betreffend. Ostwalds Klassiker der exakten Wissenschaften, Reprint der Bände 11, 24 und 25, aus dem Italienischen und Lateinischen übers. u. hrsg. v. A. v. Oettingen. Frankfurt a.M.: Deutsch.

Habermas, J. (1968), Erkenntnis und Interesse, In J. Habermas, Technik und Wissenschaft als ,Ideologie“ (S. 146-168). Frankfurt a.M.: Suhrkamp.

Hopkins, C. (2012). Reflections on 20+ Years of ESD, Journal for Education for Sustainable Development 6, 21-34.

Jullien, F. (1999). Über die Wirksamkeit. Berlin: Merve.

Jullien, F. (2004). Über die »Zeit«. Elemente einer Philosophie des Lebens. Zürich: diaphanes.

Jullien, F. (2006). Vortrag vor Managern über Wirksamkeit und Effizienz in China und im Westen. Berlin: Merve.

Jullien, F. (2008a). Umweg über China, In D. Baecker, F. Jullien, P. Jousset, W. Kubin \& P. Pörtner, Kontroverse über China. Sino-Philosophie (S. 7-30). Berlin: Merve.

Jullien, F. (2008b). Eine Dekonstruktion von außen. In D. Baecker, F. Jullien, P. Jousset, W. Kubin \& P. Pörtner. Kontroverse über China. Sino-Philosophie (S. 133159). Berlin: Merve.

Jullien, F. (2010). Die stillen Wandlungen. Berlin: Merve.

Kokemohr, R. (1989). Bildung als Begegnung? Logische und kommunikationstheoretische Aspekte der Bildungstheorie Erich Wenigers und ihre Bedeutung für biographische Bildungsprozesse in der Gegenwart, In O. Hansmann \& W. Marotzki (Hrsg.), Diskurs Bildungstheorie II: Problemgeschichtliche Orientierungen (S. 327-373). Weinheim: Deutscher Studienverlag.

Kokemohr, R. (1992). Zur Bildungsfunktion rhetorischer Figuren. Sprachgebrauch und Verstehen als didaktisches Problem, In Hartmut Entrich \& Lothar Staeck (Hrsg.), Sprache und Verstehen im Biologieunterricht (S. 16-30). Alsbach/Bergstraße: Leuchtturm.

Kokemohr, R. (2007). Bildung als Welt- und Selbstentwurf im Anspruch des Fremden. Eine theoretisch-empirische Annäherung an eine Bildungstheorie, In H.-C. Koller, W. Marotzki \& O. Sanders (Hrsg.), Bildungsprozesse und Fremdheitserfahrung. Beiträge zu einer Theorie transformatorischer Bildungsprozesse (S. 13-68). Bielefeld: Transkript.

Koller, H.-C. (2012). Bildung anders denken. Einführung in die Theorie transformatorischer Bildungsprozesse. Stuttgart: Kohlhammer.

Kapelari, Suzanne (Hg.), Vierte „Tagung der Fachdidaktik“ 2019:

„Interdisziplinäre fachdidaktische Diskurse zur Bildung für nachhaltige Entwicklung“

(C) 2020 innsbruck university press, ISBN 978-3-99106-019-2, DOI 10.15203/99106-019-2 
Kosler, T. (2016). Naturwissenschaftliche Bildung im Elementar- und Primarbereich. Zum naturwissenschaftlichen Denken mit Kindern im Kontext einer nachhaltigen Entwicklung. Bad Heilbrunn: Klinkhardt.

Kosler, T. (2018a). Repräsentieren als naturwissenschaftliche Handlungsweise. Potentiale für den Sachunterricht, In U. Franz, H. Giest, A. Hartinger, A. HeinrichDönges \& B. Reinhoffer (Hrsg.), Handeln im Sachunterricht - konzeptionelle Begründungen und empirische Befunde (S. 85-92). Bad Heilbrunn: Klinkhardt.

Kosler, T. (2018b). Naturwissenschaftliches Denken im Kontrast zu chinesischem Naturdenken, In C. Maurer (Hrsg.), Qualitätsvoller Chemie- und Physikunterricht normative und empirische Dimensionen (S. 332-335). Gesellschaft für Didaktik der Chemie und Physik. Jahrestagung in Regensburg. http://www.gdcp.de/images/tagungsbaende/GDCP Band38.pdf

Kuhn, T. (1976). Die Struktur wissenschaftlicher Revolutionen. Frankfurt a. M.: Suhrkamp.

Kuhn, T. (1981). Was sind wissenschaftliche Revolutionen? München: Carl-Friedrichvon-Siemens-Stiftung.

Lyotard, J.-F. (1986). Das postmoderne Wissen. Ein Bericht. Graz: Böhlau.

Lyotard, J.-F. (1989). Der Widerstreit. München: Fink.

Marotzki, W. (1990). Entwurf einer strukturalen Bildungstheorie. Biographietheoretische Auslegung von Bildungsprozessen in hochkomplexen Gesellschaften. Weinheim: Deutscher Studien Verlag.

Matthews, M. (2004), Thomas Kuhn's impact on science education: What lessons can be learned? Science Education, 88 (1), 90-118.

Mezirow, J. (1978a). Education for Perspective Transformation: Women's Re-entry Programs in Community Colleges. New York: Teachers College, Columbia University.

Mezirow, J. (1978b). Perspective transformation. Adult Education, 28(2), 100-110.

Mezirow, J. (1981). A critial theory of adult learning and education. Adult Education, 32(1), 3-24.

Mezirow, J. (1997). Transformative Erwachsenenbildung. Baltmannsweiler: Schneider.

Netz, R. (1999). The Shaping of Deduction in Greek Mathematics. A Study in Cognitive History. Cambridge: Cambridge University Press.

Österreichische UNESCO-Kommission (2019). Positionspapier zur Umsetzung von SDG 4 in Österreich. https://www.unesco.at/fileadmin/Redaktion/Publikationen/PublikationsDokumente/2019 Positionspapier OEUK Fachbeirat Transformative Bildung.pdf

O'Sullivan, E. (1999). Transformative Learning. Educational Vision for the 21. Century. London: Zed books.

Scott, W., \& Gough, S. (2003). Sustainable Development and Learning. Framing the issues. London: RoutledgeFalmer.

Kapelari, Suzanne (Hg.), Vierte „Tagung der Fachdidaktik“ 2019:

„Interdisziplinäre fachdidaktische Diskurse zur Bildung für nachhaltige Entwicklung“

(C) 2020 innsbruck university press, ISBN 978-3-99106-019-2, DOI 10.15203/99106-019-2 
Searle, J. (1969). Speech Acts: An Essay in the Philosophy of Language. London: Cambridge University Press.

Singer-Brodowski, M. (2016a). Transformative Bildung durch transformatives Lernen. Zur Notwendigkeit der erziehungswissenschaftlichen Fundierung einer neuen Idee. Zeitschrift für internationale Bildungsforschung und Entwicklungspädagogik, 1/2016, 13-17.

Singer-Brodowski, M. (2016b). Transformatives Lernen als neue Theorie-Perspektive in der BNE. In Umweltdachverband GmbH (Hrsg.), Jahrbuch Bildung für nachhaltige Entwicklung - Im Wandel (S. 140-139). Wien: Forum Umweltbildung im Umweltdachverband.

Sterling, S. (2001). Sustainable Education. Re-visioning Learning and Change. Cambridge: Green Books.

Sterling, S. (2003). Whole Systems Thinking as a Basis for Paradigm Change in Ecuation: Explorations in the Eontext of Sustainability. http://www.bath.ac.uk/cree/sterling/sterlingthesis.pdf

Sterling, S. (2008). Sustainable Education - Towards a deep learning response to unsustainability. Policy \& Practice: Education for Sustainable Development, 63-68.

Sterling, S. (2011). Transformative Learning and Sustainability: sketching the conceptual ground. Learning and Teaching in Higher Education, 5, 17-33.

Sterling, S. (2014). Separate Tracks or Real Synergy? Achieving a Closer Relationship between Education and SD, Post-2015. Journal of Education for Sustainable Development, 8(2), 89-112.

Vare, P., \& Scott, W. (2007). Learning for a Change: Exploring the Relationship Between Education and Sustainable Development. Journal of Education for Sustainable Development, 1(2), 191-198.

Wals, A. (2010). Mirroring, Gestaltswitching and transformative social learning. Stepping stones for developing sustainability competence, International Journal of Sustainability in Higher Education, 11(4), 380-390.

Wals, A., \& Dillon, J. (2013). Conventional and Emerging Learning Theories. Implications and Choices for Educational Researchers With a Planetary Consciousness, In R.B. Stevenson, M. Brody, J. Dillon, \& A.E.J. Wals (Eds.), Interantional Handbook of Research on Environmental Education (S. 253-161). New York: Routledge.

Kapelari, Suzanne (Hg.), Vierte „Tagung der Fachdidaktik“ 2019:

„Interdisziplinäre fachdidaktische Diskurse zur Bildung für nachhaltige Entwicklung“

(C) 2020 innsbruck university press, ISBN 978-3-99106-019-2, DOI 10.15203/99106-019-2 


\title{
Eine interdisziplinäre Studie zum Einfluss von TBLT ${ }^{1}$ auf die Scientific Interlanguage von SchülerInnen im Biologie- und Physikunterricht
}

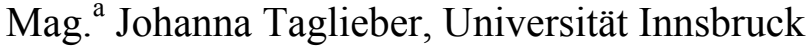 \\ Mag. ${ }^{\text {a }}$ Corinna Pieber, Universität Innsbruck \\ Univ.-Prof. ${ }^{\text {in }}$ Mag. ${ }^{\text {a }}$ Dr. ${ }^{\text {in }}$ Suzanne Kapelari, MA, Universität Innsbruck \\ Assoz.-Prof. Mag. Dr. Wolfgang Dür, Universität Innsbruck \\ Univ.-Prof. ${ }^{\text {in }}$ Mag. ${ }^{a}$ Dr. ${ }^{\text {in }}$ Barbara Hinger, MA, Universität Innsbruck
}

\section{Einleitung}

Im Jahr 2015 haben sich die Mitgliedstaaten der United Nations (UN) auf 17 Sustainable Development Goals (SDGs) verständigt. Mit der Formulierung des vierten Ziels ,Quality Education “ haben sich die UN dabei auch eine ,inklusive, gleichberechtigte und hochwertige Bildung“ zum Ziel gesetzt, die „Möglichkeiten lebenslangen Lernens“ fördern soll. Durch das Unterziel 4.7 soll sichergestellt werden, „dass alle Lernenden die notwendigen Kenntnisse und Qualifikationen zur Förderung nachhaltiger Entwicklung erwerben“ (Bundeskanzleramt Österreich, 2019).

Für den vorliegenden Beitrag ergibt sich daraus die allgemeine Frage, welche Kenntnisse und Qualifikationen für eine Bildung für nachhaltige Entwicklung

1 Task-based Language Teaching

Kapelari, Suzanne (Hg.), Vierte „Tagung der Fachdidaktik“ 2019:

„Interdisziplinäre fachdidaktische Diskurse zur Bildung für nachhaltige Entwicklung“

(C) 2020 innsbruck university press, ISBN 978-3-99106-019-2, DOI 10.15203/99106-019-2 
(BNE) grundlegend sind. Außerdem gilt es zu klären, welchen Beitrag das hier vorgestellte Dissertationsprojekt zum Erwerb dieser Kenntnisse und Qualifikationen leisten kann. Zur Beantwortung dieser Fragen wird zu Beginn des Beitrags erörtert, welche Schlüsselkompetenzen SchülerInnen als Voraussetzung für nachhaltiges Handeln benötigen. Dabei wird argumentiert, dass der Erwerb einer fachspezifischen Diskurskompetenz unter Berücksichtigung der naturwissenschaftlichen Fachsprachen nicht nur eine der Voraussetzungen für den Erwerb nachhaltigkeitsbezogener Schlüsselkompetenzen ist, sondern SchülerInnen auch zur Teilhabe an der Diskussion gesellschaftlich relevanter naturwissenschaftlicher Themen (Socioscientific Issues) befähigt. Darauf aufbauend wird ein interdisziplinäres Dissertationsprojekt vorgestellt, welches die Förderung der naturwissenschaftlichen Fachsprachen zum Ziel hat. Dafür überträgt das Projekt Task-based Language Teaching (TBLT) einen bewährten Ansatz aus der Fremdsprachendidaktik auf den Biologie- und Physikunterricht. Der Beitrag stellt die ersten Ergebnisse dieses Dissertationsprojekts vor und setzt sie zu den eingangs beschriebenen Kenntnissen und Qualifikationen in Beziehung.

\section{Beitrag einer Fachsprachenförderung zur BNE}

Wie einführend erläutert, ergibt sich aus dem SDG 4.7 die grundlegende Frage, welche Kenntnisse und Qualifikationen SchülerInnen im Sinne einer BNE erwerben müssen. Nicht erst seit der Formulierung der 17 nachhaltigen Entwicklungsziele (für eine ausführliche Beschreibung siehe Rieckmann in diesem Band) sind zahlreiche Publikationen dieser Frage auf den Grund gegangen. Dabei wurden Sammelbegriffe wie die ,Gestaltungskompetenz' nach de Haan (2002) eingeführt, um jene Kompetenz zu beschreiben, die es Lernenden ermöglicht „die Zukunft von Sozietäten, in denen man lebt, in aktiver Teilhabe im Sinne nachhaltiger Entwicklung modifizieren und modellieren zu können“ (de Haan \& Harenberg, 1999, p. 62). Auch der Frage nach Teilkompetenzen dieser übergeordneten Kompetenz wurde daraufhin bereits hinlänglich nachgegangen.

Kapelari, Suzanne (Hg.), Vierte „Tagung der Fachdidaktik“ 2019:

„Interdisziplinäre fachdidaktische Diskurse zur Bildung für nachhaltige Entwicklung“

(C) 2020 innsbruck university press, ISBN 978-3-99106-019-2, DOI 10.15203/99106-019-2 
Auf Basis einer fundierten Literaturrecherche führen Wiek, Withycombe \& Redman (2011) diese früheren Publikationen und Diskussionen zusammen und modellieren dazu eine theoretische Rahmung, welche fünf Schlüsselkompetenzen beschreibt und im sustainability research and problemsolving framework verortet: systemisches Denken, vorausschauendes Denken, normative Kompetenz, strategische Kompetenz und soziale interpersonale Kompetenz (Wiek et al., 2011, p. 205). Die AutorInnen grenzen diese Schlüsselkompetenzen in weiterer Folge zunächst von regulären Basiskompetenzen, die traditionell im (naturwissenschaftlichen) Unterricht gefördert werden sollen, $a b$. Dabei betonen sie jedoch, dass diese Basiskompetenzen - wie beispielsweise Kommunikation oder kritisches Denken - als Teil einer sog. academic sustainability education eine fundamentale Voraussetzung für den Erwerb der fünf nachhaltigkeitsbezogenen Schlüsselkompetenzen darstellen. Wiek et al. (2011) schlagen damit eine Brücke zwischen diesen und dem Kompetenzbereich ,Kommunikation“. Letzterer gewinnt vor allem in den vergangenen Jahren zunehmend an unterrichtlicher Relevanz. Gründe dafür sind nicht nur die zunehmende kulturelle und sprachliche Heterogenität der Gesellschaft (siehe Peskoller in diesem Band), sondern auch bildungspolitische Bestimmungen wie die deutschen Bildungsstandards (KMK, 2004) sowie die Prüfungsordnung der Vorwissenschaftlichen Arbeit (VWA) in Österreich. Im Rahmen der VWA müssen SchülerInnen u.a. „Kommunikations- und Diskursfähigkeit unter Beweis“ stellen (Bundesgesetzblatt II, 2012/174). Im Sinne eines Science across the Curriculum (SAC)-Ansatzes sind für diesen sprachlichen Kompetenzaufbau nicht nur der erst- und fremdsprachliche Sprachenunterricht, sondern auch andere ,nicht-sprachliche' Fächer verantwortlich (Vollmer, 2006). Dazu müssen laut Vollmer (2006) vor allem fachspezifische Wege des Denkens und Kommunizierens gefördert werden. Da diese fachspezifische Kommunikation in den Naturwissenschaften unter Verwendung von Fachsprachen stattfindet (Hoffmann, 1985), rückt der vorliegende Beitrag die fachsprachliche Kompetenz der SchülerInnen in den Vordergrund.

Kapelari, Suzanne (Hg.), Vierte „Tagung der Fachdidaktik“ 2019:

„Interdisziplinäre fachdidaktische Diskurse zur Bildung für nachhaltige Entwicklung“

(C) 2020 innsbruck university press, ISBN 978-3-99106-019-2, DOI 10.15203/99106-019-2 
Rincke (2010) bezeichnet die fachsprachliche Kompetenz von SchülerInnen als Scientific Interlanguage und beschreibt sie, Selinker (1972) folgend, als individuelles Sprachsystem zwischen Erstsprache (hier Alltagssprache) und Zielsprache (hier Fachsprachen). Das hier im dritten Abschnitt vorgestellte interdisziplinäre Dissertationsprojekt setzt sich zum Ziel die Scientific Interlanguage - im Sinne des oben beschriebenen SAC-Ansatzes - im Biologieund Physikunterricht zu fördern.

Die gezielte Förderung der Scientific Interlanguage soll einen Beitrag dazu leisten, dass SchülerInnen sowohl eine Conceptual Literacy als auch eine fachspezifische Diskurskompetenz erwerben. Vollmer (2006, p. 7) definiert Conceptual Literacy als ,the ability to think clearly with the help of language" und fachspezifische Diskurskompetenz als die Fähigkeit „to apply linguistic abilities acquired for the purpose of communicating clearly about relevant topics and thematic structures". Damit unterstreicht er die Relevanz von Fachsprachenförderung auf kognitiver (inhaltlicher) wie auch kommunikativer (sprachlicher) Ebene. SchülerInnen sollen ihre Scientific Interlanguage demnach so (weiter-)entwickeln, dass eine fachlich und sprachlich adäquate Auseinandersetzung mit gesellschaftlich relevanten naturwissenschaftlichen Themen möglich wird. Der Erwerb von Conceptual Literacy und fachspezifischer Diskurskompetenz sei laut Vollmer (2006, p. 5) daher nicht als Luxus, sondern als Türöffner zur Teilhabe an der Diskussion gesellschaftlich relevanter Themen zu betrachten. Anton (2010, p. 64) sieht darin auch eine demokratiepolitische Relevanz: Eine ausreichende fachsprachliche Kompetenz der SchulabgängerInnen verhindere eine sprachliche Abschottung naturwissenschaftlicher Fachkulturen, was der Verbreitung naturwissenschaftlicher Erkenntnisse in der Gesellschaft zugute kommen könnte. Das Verstehen naturwissenschaftlicher Fachsprachen sei damit eine Voraussetzung für ,eine demokratische Selektion dessen, was Wissenschaften ermitteln, bezüglich dessen, was die Gesellschaft benötigt und was sie weiterbringt.“

Vollmer (2006, pp. 5-6) argumentiert weiter, dass der Erwerb von Conceptual Literay und fachspezifischer Diskurskompetenz einen Beitrag zur 
Mehrsprachigkeit [im Sinne einer inneren Mehrsprachigkeit nach Wandruszka (1975)] leiste, indem die sprachlichen Varietäten innerhalb der gleichen Sprache (hier Deutsch) erweitert werden. Durch ihre Mehrsprachigkeit (innerhalb und außerhalb ihrer Erstsprache) würden SchülerInnen eine inter- und intrakulturelle Sensibilisierung erfahren, die sie wiederum $\mathrm{zu}$ einer demokratischen und aktiven Mitgestaltung Europas befähige.

Kugelmeyer \& Schecker (2013, p. 238) weisen darauf hin, dass die Vermittlung und das Erlernen von Fachinhalten nicht automatisch dazu befähigen, Fachinhalte angemessen $\mathrm{zu}$ kommunizieren. Im Kontext der VWAVorbereitung wird die Notwendigkeit der fachspezifischen (fach-)sprachlichen Vorbereitung besonders deutlich. Die VWA stellt „die umfangreichste und komplexeste Schreibaufgabe [dar], die SchülerInnen während ihrer Schullaufbahn bewältigen müssen“ (Kalcher, 2017, p. 8). Schreilechner (2012, S. 164) empfiehlt daher, im Zuge der gymnasialen Oberstufe, einen „systematischen Kompetenzaufbau” zu unterstützen. Fächerübergreifende Vorbereitungsmaßnahmen für die VWA, welche nicht auf fachspezifische Inhalte und Ausdrucksweisen eingehen, greifen dabei zu kurz (Kniffka \& Roelcke, 2016). Auch Bushati, Ebner, Niederdorfer \& Schmölzer-Eibinger (2018, p. 113) stellen fest, dass die Qualität sprachlicher VWAVorbereitungsmaßnahmen durch die Ergänzung einer intensiven fachlichen Auseinandersetzung gesteigert werden kann.

\section{Dissertationsprojekt}

Der fachsprachliche Kompetenzaufbau muss also in einem systematischen Prozess auch im naturwissenschaftlichen Fachunterricht der gymnasialen Oberstufe erfolgen. Das hier vorgestellte interdisziplinäre Dissertationsprojekt beschäftigt sich mit der Entwicklung, Implementierung und fachdidaktischen Evaluierung von Unterrichtsangeboten zur Förderung der Scientific Interlanguage im Biologie- und Physikunterricht und bettet diese in den Kontext der (fach-)sprachlichen VWA-Vorbereitung ein. Es wird davon

Kapelari, Suzanne (Hg.), Vierte „Tagung der Fachdidaktik“ 2019:

„Interdisziplinäre fachdidaktische Diskurse zur Bildung für nachhaltige Entwicklung“

(C) 2020 innsbruck university press, ISBN 978-3-99106-019-2, DOI 10.15203/99106-019-2 
ausgegangen, dass der Fachsprachenerwerb mit dem Fremdsprachenerwerb vergleichbar ist (Rincke, 2010). Dies führt dazu, einen bewährten Ansatz aus der Fremdsprachendidaktik - das Task Based Language Teaching (TBLT) - auf den Biologie- und Physikunterricht zu übertragen. Dabei soll untersucht werden, wie sich Aufgaben, die den TBLT-Prinzipien folgen, auf die Scientific Interlanguage von SchülerInnen auswirken. Ein wesentliches Prinzip des TBLT ist es, dass sich die Lernenden mit authentischen Aufgabenstellungen befassen und durch den Einsatz und Erwerb rezeptiver und/oder produktiver Sprachkompetenzen inhaltlich engagiert ein kommunikatives Ziel erreichen (Ellis, 2003). Um die Grundlage für die Entwicklung authentischer Unterrichtsangebote zu schaffen und auf reale (fach-)sprachliche Bedürfnisse der Lernenden eingehen zu können, wurde zu Beginn des Projekts eine taskbased Needs Analysis (NA) durchgeführt.

\section{Needs Analysis}

\subsection{Forschungsinteresse}

Ziel der vorliegenden NA ist es, (fach-)sprachliche Bedürfnisse von SchülerInnen im naturwissenschaftlichen Unterricht $\mathrm{zu}$ identifizieren. Dabei wird der Fokus auf die derzeitige Umsetzung der VWA in Tiroler Allgemeinbildenden Höheren Schulen (AHS) gelegt. Die folgenden Forschungsfragen leiten die NA:

F1 Welche (fach-)sprachlichen Aufgaben bearbeiten SchülerInnen beim Erstellen der VWA?

F2 Welche (fach-)sprachlichen Herausforderungen ergeben sich beim Erstellen der VWA?

F3 Wie werden SchülerInnen aktuell (fach-)sprachlich auf die VWAErstellung vorbereitet? 


\subsection{Untersuchungsdesign}

Die vorliegende NA orientiert sich methodisch an Serafini, Lake \& Long (2015). Diese empfehlen, unterschiedliche Quellen unter Anwendung verschiedener Methoden zu konsultieren, um eine Triangulierung der Daten und somit eine fundierte Bedarfsanalyse zu ermöglichen. Die Verwendung des Tasks als Analyseeinheit gewährleistet eine kohärente Vorgehensweise von der NA über die Task-Erstellung bis zur Implementierung.

Konkret erhebt die vorliegende Untersuchung die Perspektive von SchülerInnen sowie Lehrpersonen auf (fach-)sprachliche Aufgaben und Herausforderungen der VWA im naturwissenschaftlichen Bereich sowie deren Wahrnehmung der derzeitigen Vorbereitungsmaßnahmen für die VWA inner- und außerhalb des naturwissenschaftlichen Fachunterrichts. Da hierzu keine Forschungsergebnisse spezifisch für den österreichischen Kontext vorliegen, wurde ein exploratives mixed methods Design nach Kuckartz (2014, p. 81) gewählt. Leitfadeninterviews mit SchülerInnen und Lehrpersonen liefern dabei die Grundlage zur Erstellung von Onlinefragebögen zur Beantwortung der Forschungsfragen.

\subsection{Qualitative Leitfadeninterviews}

Die Interviewleitfäden für SchülerInnen und Lehrpersonen wurden im Forschungsteam entwickelt und nach einer Pilotierungsphase $\left(\mathrm{N}_{\mathrm{SuS}}=2, \mathrm{~N}_{\mathrm{LP}}=3\right)$ überarbeitet. Die Interviews können nach Bortz \& Döring (2002, p. 238) als halb-strukturierte direkte Einzelinterviews mit ermittelnder Funktion in einem neutralen Setting charakterisiert werden.

Die Interviews wurden in den Schuljahren 2016/17, 2017/18 und 2018/19 durchgeführt. In diesem Zeitraum nahmen insgesamt 15 SchülerInnen an einer Tiroler AHS teil, welche ihre VWA im naturwissenschaftlichen Bereich verfasst hatten. Darüber hinaus wurden 11 Lehrpersonen naturwissenschaftlicher Fächer

Kapelari, Suzanne (Hg.), Vierte ,Tagung der Fachdidaktik“ 2019:

„Interdisziplinäre fachdidaktische Diskurse zur Bildung für nachhaltige Entwicklung“

(C) 2020 innsbruck university press, ISBN 978-3-99106-019-2, DOI 10.15203/99106-019-2 
an sechs Tiroler Schulen (alle AHS) befragt. SchülerInnen und Lehrpersonen wurden über persönliche Kontakte zu den Schulen rekrutiert. Darüber hinaus wurden die LeiterInnen der Arbeitsgemeinschaften für die Fächer Biologie und Physik gebeten, eine Einladung zur Teilnahme an alle Tiroler Lehrpersonen weiterzuleiten.

Alle Interviews wurden digital aufgezeichnet, anschließend nach dem einfachen Transkriptionssystem nach Dresing \& Pehl (2015) transkribiert und mit Hilfe des Computerprogramms MAXQDA unter Heranziehung der qualitativen Inhaltsanalyse nach Mayring (2015) ausgewertet. Die Analysekategorien wurden in Kodierkonferenzen im Forschungsteam induktiv gebildet und diskutiert, um die Intercoderreliabilität der Analyse zu erhöhen.

\subsection{Onlinefragebogen}

Auf Basis der Ergebnisse der Leitfadeninterviews wurden zwei Onlinefragebögen entwickelt (Bühner, 2011): ein SchülerInnen- und ein LehrerInnenfragebogen. Dabei wurde die Onlineplattform https://www.soscisurvey.de/ verwendet. In der Pilotierungsphase $\left(\mathrm{N}_{\mathrm{SuS}}=23\right.$, $\mathrm{N}_{\mathrm{LP}}=3$ ) wurde die Formulierung und Auswahl der Items durch Think-Aloud Protokolle (Collins, 2003) und statistische Testanalysen überarbeitet. Der Onlinelink zu den Fragebögen wurde mit der Bitte um Weiterleitung an die DirektorInnen aller Tiroler AHSen geschickt, woraufhin die SchülerInnen und Lehrpersonen freiwillig an der Studie teilnehmen konnten. An der Fragebogenuntersuchung im Schuljahr 2018/19 nahmen 119 SchülerInnen und 45 Lehrpersonen in Tirol teil.

Die teilnehmenden SchülerInnen (71 weiblich, 42 männlich) waren zum Zeitpunkt der Befragung 17 bis19 Jahre alt und hatten den schriftlichen Teil ihrer VWA bereits abgeschlossen und eingereicht, jedoch noch keine finale Beurteilung erhalten. Die Auswahl dieses Befragungszeitraums minimiert die Beeinflussung der Selbsteinschätzung der SchülerInnen durch die erhaltene 
Note. Bezüglich der eigenen Sprachlernbiographie gab der Großteil $(92,4 \%)$ der SchülerInnen an, monolingual aufgewachsen zu sein. 115 SchülerInnen nannten Deutsch, sechs Türkisch, vier Kroatisch und sechs ,Sonstige' als (eine) ihre(r) Erstsprache(n).

Die befragten Lehrpersonen ( 26 weiblich, 16 männlich, 3 keine Angabe) waren überwiegend erfahrene Lehrkräfte mit mehr als 20 Dienstjahren (53,3\%). 31,1\% waren zum Zeitpunkt der Befragung 11 bis 20 Dienstjahre tätig und 15,6\% unter zehn Jahre. Alle Lehrpersonen hatten zum Zeitpunkt der Befragung bereits mindestens eine naturwissenschaftliche VWA betreut, 44 von ihnen unterrichten mindestens ein naturwissenschaftliches Fach. Die Lehrpersonen waren alle monolingual mit Deutsch als Erstsprache aufgewachsen und hatten durch zusätzlichen Fremdsprachenunterricht in Englisch (100\%), Französisch (17,9\%), Latein (51,3\%), Italienisch (17,9\%) bzw. Spanisch (10,3\%) selbst Erfahrungen beim Erlernen einer Fremdsprache gewonnen.

\subsection{Ergebnisse und Diskussion}

Im Anschluss werden einige aus den Daten gewonnene Erkenntnisse näher beschrieben. Dabei werden sowohl quantitative als auch qualitative Ergebnisse dargestellt und diskutiert.

\subsection{1 (Fach-)sprachliche Aufgaben}

Während die Lehrpersonen den Fokus in den Interviews vor allem auf (fach-)sprachliche Herausforderungen einer (naturwissenschaftlichen) VWA legen, beschreiben die SchülerInnen zum Teil sehr detailliert, wie sie bei der VWA-Erstellung vorgegangen sind und welche (fach-)sprachlichen Aufgaben sie dabei bearbeitet haben. Diese (fach-)sprachlichen Arbeitsschritte (hier Target Tasks) wurden in der Analyse übergeordneten Arbeitsphasen (hier Target Task Types) zugeordnet. Dabei wurde deutlich, dass die SchülerInnen im Zuge der VWA-Erstellung den folgenden (vor-)wissenschaftlichen

Kapelari, Suzanne (Hg.), Vierte „Tagung der Fachdidaktik“ 2019:

„Interdisziplinäre fachdidaktische Diskurse zur Bildung für nachhaltige Entwicklung“

(C) 2020 innsbruck university press, ISBN 978-3-99106-019-2, DOI 10.15203/99106-019-2 
Arbeitsprozess durchlaufen: Themenfindung - Literaturrecherche - ev. eigene Forschungsarbeit - Schreibprozess - Überarbeitung. Tab. 1 zeigt ausgewählte Target Tasks, die SchülerInnen nach eigener Angabe im Zuge dessen durchführen:

Tab. 1: Ausgewählte Ergebnisse der SchülerInneninterviews zu Target Tasks und Target Task Types

\begin{tabular}{|l|l|}
\hline Target Task Types & Target Tasks \\
\hline Themenfindung & $\begin{array}{l}\text { Forschungsfragen formulieren } \\
\text { den Erwartungshorizont verfassen }\end{array}$ \\
\hline Literaturrecherche & $\begin{array}{l}\text { die Verlässlichkeit von Quellen beurteilen } \\
\text { Zusammenhänge zwischen verschiedenen Quellen herstellen }\end{array}$ \\
\hline Schreibprozess & $\begin{array}{l}\text { naturwissenschaftliche Sachverhalte schriftlich erklären } \\
\text { Wissenschaftssprache verwenden }\end{array}$ \\
\hline
\end{tabular}

\subsection{2 (Fach-)sprachliche Herausforderungen}

In den Fragebögen wurden sowohl SchülerInnen als auch Lehrpersonen gebeten, die Schwierigkeit der Target Tasks und Target Task Types auf einer vierstufigen Likert-Skala zu bewerten (vgl. Abb. 1). 


\section{Kreuze an, inwieweit folgende Aussagen auf dich zutreffen.}

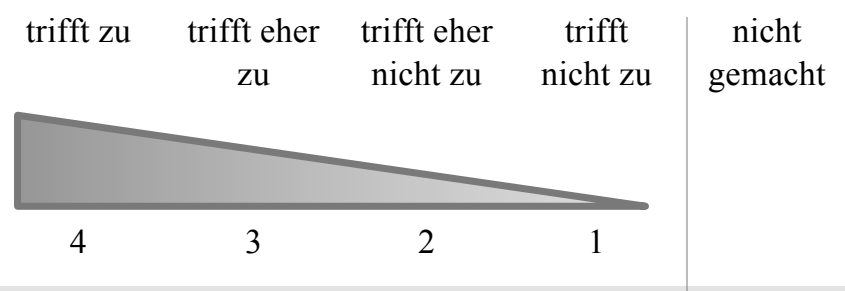

\section{Es war einfach für} mich...

... Leitfragen zu

formulieren

Abb. 1: Beispiel SchülerInnenfragebogen - Aufgaben (Eigene Darstellung J.T.)

Die Mittelwerte $(\overline{\mathrm{x}})$ aller Items liegen über der Mitte der Ratingskala $(\mu=2,5)$ (vgl. Tab. 2). Alle Target Tasks und Target Task Types werden somit von SchülerInnen und Lehrpersonen durchschnittlich als ,einfach' oder ,eher einfach` eingestuft. Die Häufigkeit der einzelnen Antworten zeigt jedoch, dass es bei vielen Items einen relativ hohen Anteil an SchülerInnen gibt, die die genannten Aufgaben als ,eher schwierig' oder ,schwierig' einstufen (vgl. Tab. 2). Die Aussage ,Der Schreibprozess war einfach für mich` trifft beispielsweise auf über $40 \%$ der SchülerInnen, eher nicht` oder, nicht` zu. 
Tab. 2: Ausgewählte Ergebnisse der Fragebogenuntersuchung zur Schwierigkeit von Target Task Types

\begin{tabular}{|c|c|c|c|c|c|c|c|c|c|c|}
\hline \multirow{3}{*}{$\begin{array}{c}\text { Item im } \\
\text { Fragebogen }\end{array}$} & \multirow{2}{*}{\multicolumn{2}{|c|}{ Mittelwert }} & \multicolumn{8}{|c|}{ Antworthäufigkeit in \% } \\
\hline & & & \multicolumn{2}{|c|}{$\begin{array}{c}4 \\
\text { trifft nicht } \\
z u\end{array}$} & \multicolumn{2}{|c|}{$\begin{array}{c}3 \\
\text { trifft eher } \\
\text { nicht } z u\end{array}$} & \multicolumn{2}{|c|}{$\begin{array}{c}2 \\
\text { trifft eher } \\
z u\end{array}$} & \multicolumn{2}{|c|}{$\begin{array}{c}1 \\
\text { trifft } z u\end{array}$} \\
\hline & $\mathrm{SuS}$ & LP & $\mathrm{SuS}$ & LP & $\mathrm{SuS}$ & LP & $\mathrm{SuS}$ & LP & $\mathrm{SuS}$ & LP \\
\hline $\begin{array}{l}\text { Die } \\
\text { Themenfindung } \\
\text { war einfach. }\end{array}$ & 2,82 & 3,53 & 11,8 & 2,2 & 27,7 & 2,2 & 27,7 & 35,6 & 32,8 & 60,0 \\
\hline $\begin{array}{l}\text { Die Literatur- } \\
\text { recherche war } \\
\text { einfach. }\end{array}$ & 2,86 & 2,96 & 8,4 & 2,2 & 26,1 & 26,7 & 37,0 & 44,4 & 28,6 & 26,7 \\
\hline $\begin{array}{l}\text { Der } \\
\text { Schreibprozess } \\
\text { war einfach. }\end{array}$ & 2,66 & 2,80 & 10,1 & 6,7 & 31,1 & 24,4 & 42,0 & 48,9 & 16,8 & 17,8 \\
\hline
\end{tabular}

Im Vergleich mit den Leitfadeninterviews wird deutlich, dass Herausforderungen u.a. durch die den SchülerInnen unbekannten Fachsprachen entstehen. Beispielsweise werden Schwierigkeiten beim Verstehen naturwissenschaftlicher Fachliteratur genannt:

„[E]s ist ja Fachliteratur, wo verschiedene Begriffe sind, die man eigentlich nicht kennt und es ist ganz eine andere Sprache. Man liest solche Bücher nicht in der Schule. Das habe ich schwierig gefunden, weil es ist ein WISSENSCHAFTLICHES Buch und nicht eines mit Einleitung, Hauptteil, Schluss.“ (S1)

Die naturwissenschaftlichen Fachsprachen scheinen somit eine ,ganz andere Sprache', in anderen Worten, wie eine Fremdsprache' zu sein. Ursachen für diese Schwierigkeit der Rezeption wissenschaftlicher Texte werden in der Fachliteratur (z.B. Fang, 2006) vor allem durch die Besonderheiten naturwissenschaftlicher Fachsprachen auf Wort-, Satz- und Textebene erklärt. S1 scheint in der Schule nicht hinreichend auf diese Besonderheiten vorbereitet 
worden zu sein. Auch andere SchülerInnen sprechen diese mangelnde Vorbereitung an:

„Vom Sprachlichen her, dass man es richtig formuliert, dass es schon richtig Deutsch ist, aber auch wissenschaftlich klingt und nicht nur so dahergeredet. Das war noch eine große Umstellung, weil man in der Schule vorher nie solche Texte geschrieben hat.“ $\left(\mathrm{S}_{\mathrm{P}} 2\right)$

Auch die interviewten Lehrpersonen berichten von zahlreichen (fach-) sprachlichen Herausforderungen, die sich im naturwissenschaftlichen Kontext vor allem durch die enge Verbindung von Sprache und Inhalt ergeben. Trotzdem sei ein gutes inhaltliches Verstehen keine automatische Voraussetzung für eine gute sprachliche Kompetenz:

„Ich habe Schüler ${ }^{2}$, die [...] inhaltlich [...] sehr gut sind, das Thema verstanden haben, durchgekaut haben, aber Probleme haben, das einfach gescheit zu Papier zu bringen.“(LP9)

Dieser Befund deckt sich mit den Ergebnissen einer Studie von Kugelmeyer \& Schecker (2013) zur Kommunikationskompetenz in Physik. Sie zeigt, dass „Fachwissen nur bis zu einem gewissen Grad einen förderlichen Einfluss auf Kommunikationskompetenz hat" (p. 235) und sehr hohes Fachwissen somit kein Garant für eine hohe Kommunikationskompetenz zu sein scheint.

Es zeigt sich außerdem, dass sich eher SchülerInnen, die eine hohe Selbstwirksamkeitserwartung haben, dafür entscheiden, eine naturwissenschaftliche VWA zu schreiben. 70,6\% der befragten SchülerInnen würden ihre VWA mit ,Sehr gut ${ }^{\star}$ oder ,Gut' beurteilen und 80,6\% der SchülerInnen schätzen ihre schriftlichen Fähigkeiten in Deutsch als ,Sehr gut' oder ,Gut" ein. Die qualitativen und quantitativen Ergebnisse der Befragung von Lehrpersonen stützen diese Annahme. LP5 meint beispielsweise, dass ,gerade in Physik das

2 Die Interviewausschnitte wurden wörtlich und somit ungeachtet der Verwendung einer geschlechtergerechten Sprache übernommen.

Kapelari, Suzanne (Hg.), Vierte „Tagung der Fachdidaktik“ 2019:

„Interdisziplinäre fachdidaktische Diskurse zur Bildung für nachhaltige Entwicklung“

(C) 2020 innsbruck university press, ISBN 978-3-99106-019-2, DOI 10.15203/99106-019-2 
eigentlich ein sehr überschaubarer Kreis ist und die Schüler, die in Physik [...] eine VWA schreiben wollen, zum Glück so gut wie immer herausragende Voraussetzungen mitbringen“. Eine kontinuierliche (fach-)sprachliche Vorbereitung auf die VWA im naturwissenschaftlichen Unterricht wäre daher nicht nur, aber vor allem für jene SchülerInnen wichtig, die keine ,herausragenden Voraussetzungen' mitbringen.

\subsection{3 (Fach-)sprachliche Vorbereitungsmaßnahmen}

Sowohl die Interviews als auch die Fragebogenuntersuchung zeigen, dass SchülerInnen aktuell vor allem durch fächerübergreifende Angebote (z.B. VWA-Workshops) auf die VWA vorbereitet werden. Dort werden vor allem grundsätzliche wissenschaftliche Arbeitstechniken, Zitieren und Formatieren besprochen. Im Fragebogen geben $89,1 \%$ der SchülerInnen an, dass es an ihrer Schule derartige fächerübergreifende Vorbereitungsangebote gibt.

Eine Vorbereitung im Regelunterricht der Oberstufe scheint laut Interviewdaten vor allem im Deutschunterricht und nur vereinzelt in anderen Fächern wie Informatik oder Mathematik stattzufinden. 23,5\% der SchülerInnen geben in der Fragebogenuntersuchung an, im naturwissenschaftlichen Regelunterricht der Oberstufe auf die VWA vorbereitet worden zu sein. Im Gegenschluss ist es daher nicht verwunderlich, dass sich 42,9\% der SchülerInnen nicht ausreichend auf die VWA vorbereitet fühlen.

Die Vorbereitung auf die VWA im naturwissenschaftlichen Regelunterricht scheint bei den interviewten Lehrpersonen unterschiedlichen Schwerpunktsetzungen $\mathrm{zu}$ unterliegen. Allgemein dominieren die inhaltliche und methodische Vorbereitung. Die Lehrpersonen nennen in den Interviews jedoch auch einige sprachliche Vorbereitungsmaßnahmen; darunter vor allem das Protokollieren und Auswerten von Experimenten, die Arbeit mit Fachtexten, das Beschreiben und Interpretieren von Abbildungen und Diagrammen sowie das explizite Beschreiben der Charakteristika naturwissenschaftlicher 
Erkenntnisprozesse (Nature of Science). Häufig weisen die Lehrenden darauf hin, dass sie selbst als sprachliches Vorbild dienen, eine korrekte Sprache bei der Stundenwiederholung einfordern oder auf schriftliche Tests auch fachsprachliches Feedback (ohne Einfluss auf die Note) geben. Sprachliches Aushandeln fachlicher Inhalte in mündlicher und schriftlicher Form außerhalb von Prüfungssituationen wird ebenfalls, wenn auch seltener, genannt.

In den Fragebögen wurden SchülerInnen und Lehrpersonen wiederum gebeten, die Häufigkeit einzelner Vorbereitungsmaßnahmen auf einer vierstufigen Likert-Skala zu bewerten (vgl. Abb. 2).

\section{Kreuze an, wie oft du folgende Teilaufgaben während der gesamten Oberstufe im naturwissenschaftlichen Unterricht (Biologie, Chemie, Physik) machen musstest.}

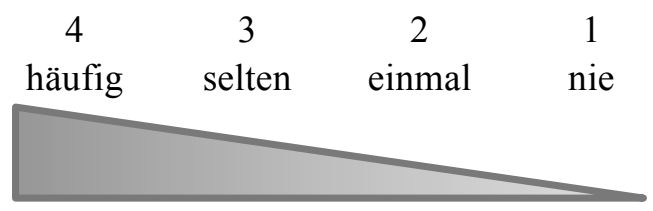

\section{Wie oft musstest du...}

... selbstständige eine

Forschungsfrage formulieren?

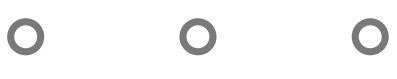

Abb. 2: Beispiel SchülerInnenfragebogen - Vorbereitung (Eigene Darstellung J.T.)

$\mathrm{Da}$ es sich hier um ordinalskalierte Werte handelt, wurden anstatt des Mittelwerts die Lageparameter Modus und Median berechnet. Tab. 3 zeigt 
ausgewählte Ergebnisse der Fragebögen (SuS und $\mathrm{LP}^{3}$ ) zur Häufigkeit spezifischer Vorbereitungsmaßnahmen.

Tab. 3: Ausgewählte Ergebnisse der Fragebogenuntersuchung zu Vorbereitungsmaßnahmen im natwi Fachunterricht

\begin{tabular}{|l|l|c|c|c|c|}
\hline Target Task Type & Vorbereitungsmaßnahme & \multicolumn{2}{|c|}{ Median } & \multicolumn{2}{|c|}{ Modus } \\
\hline \multirow{5}{*}{ Themenfindung } & $\begin{array}{l}\text { SuS } \\
\text { selbstständig eine Forschungsfrage } \\
\text { formulieren }\end{array}$ & 2 & 3 & 1 & 3 \\
\cline { 2 - 7 } & $\begin{array}{l}\text { selbstständig einen Titel für einen Text } \\
\text { finden }\end{array}$ & 3 & 2 & 1 & 1 \\
\hline Literaturrecherche & selbständig Fachliteratur recherchieren & 3 & 3 & 3 & 3 \\
\cline { 2 - 7 } & Fachliteratur lesen & 3 & 3 & 4 & 3 \\
\hline \multirow{5}{*}{ Forschungsarbeit } & $\begin{array}{l}\text { eigene Forschung mit Hilfe von } \\
\text { Fachliteratur planen }\end{array}$ & 1 & 1 & 1 & 1 \\
\cline { 2 - 7 } & $\begin{array}{l}\text { eigene Forschung in einem Text } \\
\text { beschreiben }\end{array}$ & 2 & 2 & 1 & 1 \\
\hline \multirow{3}{*}{ Schreibprozess } & in einem Text Fachbegriffe verwenden & 3 & 3 & 4 & 3 \\
\cline { 2 - 7 } & eine Abbildung beschreiben & 4 & 4 & 4 & 4 \\
\cline { 2 - 7 } & selbstständig eine Abbildung erstellen & 3 & 3 & 1 & 3 \\
\hline
\end{tabular}

Insgesamt weisen die Ergebnisse der vorliegenden NA also darauf hin, dass im naturwissenschaftlichen Fachunterricht noch keine ausreichende (fach-) sprachliche Vorbereitung auf die VWA erfolgt. Als Gründe dafür werden in den Interviews u.a. die fehlenden zeitlichen Ressourcen genannt und auf die Vielzahl der im Lehrplan vorgeschriebenen Fachinhalte verwiesen. Die sprachlich heterogene Zusammensetzung der SchülerInnen im Unterricht wird ebenfalls erwähnt. Markic (2013) sieht einen weiteren Grund darin, dass Lehrpersonen naturwissenschaftlicher Fächer oft über kein ausreichendes Wissen über die Eigenschaften der Fachsprachen bzw. methodische Kompetenz zur Förderung von Fachsprachen verfügen. Diese Variablen wurden in der vorliegenden NA nicht explizit untersucht.

3 Bei den Lehrpersonen wurden 38 Fälle berücksichtigt.

Kapelari, Suzanne (Hg.), Vierte „Tagung der Fachdidaktik“ 2019:

„Interdisziplinäre fachdidaktische Diskurse zur Bildung für nachhaltige Entwicklung“

(C) 2020 innsbruck university press, ISBN 978-3-99106-019-2, DOI 10.15203/99106-019-2 


\section{Zusammenfassung und Ausblick}

Im vorliegenden Beitrag wurde eine explorative NA zu (fach-)sprachlichen Aufgaben, Herausforderung und Vorbereitungsmaßnahmen für die VWAErstellung im naturwissenschaftlichen Kontext vorgestellt. Ausgehend von der Analyse qualitativer Leitfadeninterviews wurde eine Liste an (fach-) sprachlichen Target Tasks und Target Task Types erstellt, welche für SchülerInnen beim Erstellen einer naturwissenschaftlichen VWA relevant sind. Viele der identifizierten Target Task Types erfordern jedoch nicht nur (fach-)sprachliche Kompetenzen, sondern u.a. auch ein fundiertes Fachwissen (z.B. naturwissenschaftliche Sachverhalte schriftlich erklären) oder die Fähigkeit des Kritischen Denkens (z.B. Verlässlichkeit von Quellen beurteilen). Aufgrund der Komplexität dieser Handlungen ist zu erwarten, dass einige dieser Target Task Types für SchülerInnen eine Herausforderung darstellen. Auch wenn die Triangulierung der qualitativen und quantitativen Befragung keine eindeutige Aussage über (fach-)sprachliche Herausforderungen zulässt, zeigt die vorliegende Untersuchung, dass SchülerInnen im naturwissenschaftlichen Regelunterricht der Oberstufe keine ausreichende (fach-)sprachliche Vorbereitung erfahren. Diese Vorbereitung ist jedoch notwendig, um es auch jenen SchülerInnen die keine herausragenden fachlichen und sprachlichen Voraussetzungen mitbringen, $\mathrm{zu}$ ermöglichen, eine VWA im naturwissenschaftlichen Bereich so gut wie möglich umzusetzen. Darüber hinaus kann eine Förderung der naturwissenschaftlichen Fachsprachen zum Erwerb von Conceptual Literacy sowie fachspezifischer Diskurskompetenz beitragen. Diese bilden als Teil der Kommunikationskompetenz, gemeinsam mit anderen Basiskompetenzen, ein Fundament für die Entwicklung der fünf nachhaltigkeitsbezogenen Schlüsselkompetenzen. Sind SchülerInnen in der Lage, am fachspezifischen Diskurs teilzuhaben, können sie sich aktiv an der Diskussion gesellschaftlich relevanter Themen beteiligen und demokratische Prozesse der europäischen und globalen Zukunft mitgestalten.

In der nächsten Phase des Dissertationsprojekts werden Tasks nach TBLT für die VWA-Vorbereitung im naturwissenschaftlichen Unterricht erstellt. Dabei

Kapelari, Suzanne (Hg.), Vierte „Tagung der Fachdidaktik“ 2019:

„Interdisziplinäre fachdidaktische Diskurse zur Bildung für nachhaltige Entwicklung“

(C) 2020 innsbruck university press, ISBN 978-3-99106-019-2, DOI 10.15203/99106-019-2 
wird zu beachten sein, dass die oben beschriebenen Target Task Types nur durch ein komplexes Zusammenspiel vieler verschiedener Voraussetzungen und Kompetenzen (darunter (fach-)sprachliche Kompetenzen, Fachwissen, Kritisches Denken) bewältigt werden können. Bei der Task-Erstellung wird auf eine konsequente Verknüpfung von Sprache und Inhalt Wert gelegt, die den Sprachgebrauch in einen authentischen naturwissenschaftlichen Kontext einbettet. Anknüpfungspunkte $\mathrm{zu}$ anderen (naturwissenschaftlichen) Basiskompetenzen, wie beispielsweise dem Kritischen Denken (Rafolt, Kapelari, \& Kremer, 2019), werden gesucht. So sollen fachspezifische Wege des Denkens und Kommunizierens gefördert werden. Auch wenn das Dissertationsprojekt keinen Anspruch auf eine spezifische Förderung der eingangs beschriebenen nachhaltigkeitsbezogenen Schlüsselkompetenzen erhebt, wird durch die Förderung fundamentaler Basiskompetenzen ein Beitrag zur BNE geleistet.

\section{Literatur}

Anton, M. A. (2010). "Wie heißt das auf Chemisch?": Sprachebenen der Kommunikation im und nach dem Chemieunterricht. In G. Fenkart, A. Lembens, \& E. Erlacher-Zeitlinger (Eds.), Sprache, Mathematik und Naturwissenschaften (pp. 63-83). Innsbruck, Wien, Bozen: Studien Verlag.

Bundesgesetzblatt II (2012/174).

Bortz, J., \& Döring, N. (2002). Forschungsmethoden und Evaluation: Für Human- und Sozialwissenschaftler (3rd ed.). Heidelberg: Springer.

Bühner, M. (2011). Einführung in die Test- und Fragebogenkonstruktion (3rd ed.). Hallbergmoos: Pearson.

Bundeskanzleramt Österreich (2019). Ziele der Agenda 2030. Retrieved from https://www.bundeskanzleramt.gv.at/entwicklungsziele-agenda-2030

Bushati, B., Ebner, C., Niederdorfer, L., \& Schmölzer-Eibinger, S. (2018). Wissenschaftlich schreiben lernen in der Schule. Baltmannsweiler: Schneider Verlag Hohengehren GmbH.

Collins, D. (2003). Pretesting survey instruments: An overview of cognitive methods. Quality of Life Research, 12, 229-238.

Dresing, T., \& Pehl, T. (2015). Praxisbuch Interview, Transkription \& Analyse: Anleitungen und Regelsysteme für qualitativ Forschende (6.). Marburg: Eigenverlag. 
Ellis, R. (2003). Task-based language learning and teaching. Oxford, New York: Oxford University Press.

Fang, Z. (2006). The Language Demands of Science Reading in Middle School. International Journal of Science Education, 28(5), 491-520. Retrieved from https:/www.tandfonline.com/doi/full/10.1080/09500690500339092

Haan, G. d. (2002). Die Kernthemen der Bildung für eine nachhaltige Entwicklung. ZEP: Zeitschrift für internationale Bildungsforschung und Entwicklungspädagogik, 25(1), 13-20.

Haan, G. d., \& Harenberg, D. (1999). Bildung für eine nachhaltige Entwicklung: Gutachten zum Programm. Materialien zur Bildungsplanung und zur Forschungsförderung: Heft 72. Bonn: Bund-Länder-Kommission für Bildungsplanung und Forschungsförderung (BLK) Geschäftsstelle. Retrieved from http://www.pedocs.de/frontdoor.php?source opus $=218$

Hoffmann, L. (1985). Kommunikationsmittel Fachsprache: Eine Einführung (2nd ed.). Tübingen: Narr.

Kalcher, N. (2017). Argumentative Textprozeduren in Vorwissenschaftlichen Arbeiten (Diplomarbeit). Universität Graz.

Kniffka, G., \& Roelcke, T. (2016). Fachsprachenvermittlung im Unterricht. utb; StandardWissen Lehramt. Stuttgart, Paderborn: Ferdinand Schöningh. Retrieved from http://www.utb-studi-e-book.de/9783838540948

Kuckartz, U. (2014). Mixed Methods: Methodologie, Forschungsdesigns und Analyseverfahren. Wiesbaden: Springer VS.

Kugelmeyer, C., \& Schecker, H. (2013). Schülerinnen und Schüler erklären Physik Modellierung, Diagnostik und Förderung von Kommunikationskompetenz im Physikunterricht. In M. Becker-Mrotzek, K. Schramm, E. Thürmann, \& H. J. Vollmer (Eds.), Fachdidaktische Forschungen: Vol. 3. Sprache im Fach: Sprachlichkeit und fachliches Lernen (pp. 225-240). Münster: Waxmann.

Bildungsstandards im Fach Biologie für den Mittleren Schulabschluss (Jahrgangsstufe 10) (2004).

Markic, S. (2013). Science teachers' PCK about teaching and learning scientific language in science classes. In C. P. Constantinou, Papadouris N., \& A. Hadjigeorgious (Eds.), Science Education Research For Evidence-based Teaching and Coherence in Learning - Strand 12 (pp. 2181-2186). Nicosia, Cyprus: ESERA.

Mayring, P. (2015). Qualitative Inhaltsanalyse: Grundlagen und Techniken (12th ed.). Beltz Pädagogik. Weinheim: Beltz.

Rafolt, S., Kapelari, S., \& Kremer, K. (2019). Kritisches Denken im naturwissenschaftlichen Unterricht - Synergiemodell, Problemlage und Desiderata. Zeitschrift für Didaktik der Naturwissenschaften, 85(2), 1-13. https://doi.org/10.1007/s40573-019-00092-9

Kapelari, Suzanne (Hg.), Vierte „Tagung der Fachdidaktik“ 2019:

„Interdisziplinäre fachdidaktische Diskurse zur Bildung für nachhaltige Entwicklung“

(C) 2020 innsbruck university press, ISBN 978-3-99106-019-2, DOI 10.15203/99106-019-2 
Rincke, K. (2010). Alltagssprache, Fachsprache und ihre besonderen Bedeutungen für das Lernen. Zeitschrift für Didaktik der Naturwissenschaften, 16, 235-260.

Selinker, L. (1972). Interlanguage. International Review of Applied Linguistics in Language, 10(3), 209-231.

Serafini, E. J., Lake, J. B., \& Long, M. H. (2015). Needs analysis for specialized learner populations: Essential methodological improvements. English for Specific Purposes, $40,11-26$.

Vollmer, H. J. (2006). Language Across the Curriculum. In Europäischer Rat (Chair), Intergovernmental Conference: Languages of Schooling: towards a Framework for Europe. Symposium conducted at the meeting of Europäischer Rat, Strasbourg.

Wandruszka, M. (1975). Mehrsprachigkeit: Öffentlicher Vortrag. In H. Moser (Ed.), Sprache der Gegenwart. Schriften des Instituts für deutsche Sprache: Vol. 36. Sprachwissenschaft und Sprachdidaktik: Jahrbuch 1974 (pp. 321-350). Düsseldorf: Pädagogischer Verlag Schwann.

Wiek, A., Withycombe, L., \& Redman, C. L. (2011). Key competencies in sustainability: a reference framework for academic program development. Sustainability Science, 6(2), 203-218. https://doi.org/10.1007/s11625-011-0132-6 


\title{
Literaturadaptionen in der Graphic Novel Eine weitere Axt für das gefrorene Meer in uns?
}

\author{
Mag. . Caroline Bader, Universität Innsbruck
}

\begin{abstract}
Als Franz Kafka 1904 an Oskar Pollak schrieb, ein Buch müsse wie „die Axt sein für das gefrorene Meer in uns" ${ }^{\text {1 }}$, bezog er sich dabei auf jene Werke, denen er ein hohes Potenzial zuschrieb, etwas tief im Inneren der Menschen zu berühren. Literatur, die eine solch brachiale Wirkung auf uns ausübt, bricht etwas auf, dringt (pathetisch gesprochen) zu etwas in unserem Inneren vor, hält uns zu intensiver Lektüre an und bewirkt nachhaltiges literarisches Lesen; nur diese Werke waren es Kafka wert, gelesen zu werden.

Als Klassiker der ,Weltliteratur' genießen Kafkas Erzählungen kanonischen Status an Österreichs Schulen. Dass jedes seiner Werke $\mathrm{zu}$ intensivem, nachhaltigem literarischen Lernen anhalten kann und so gewissermaßen eine ,Axt' darstellt, die das Meer in uns aufzubrechen vermag, dürfte insofern heute kaum noch jemand bezweifeln. Für Literaturcomics und -Graphic Novels gilt dies in dieser Form noch nicht. Diese Formate leiden nach wie vor unter dem Makel des Trivialliterarischen, wodurch es in den häufigsten Fällen nicht zu einer schüler- und lehrerseitigen Auseinandersetzung mit dem Inhalt kommt, weil der Einbezug in den Unterricht schon am ,Trägermedium“ scheitert. Darüber hinaus fühlen sich eine große Anzahl von Lehrenden mit dem
\end{abstract}

1 Brief an Oskar Pollak vom 27. Jänner 1904, in: Briefe 1902-1924, Hrsg. von Max Brod. Frankfurt a.M.: 1966.

Kapelari, Suzanne (Hg.), Vierte „Tagung der Fachdidaktik“ 2019:

„Interdisziplinäre fachdidaktische Diskurse zur Bildung für nachhaltige Entwicklung“

(C) 2020 innsbruck university press, ISBN 978-3-99106-019-2, DOI 10.15203/99106-019-2 
Medienformat überfordert und zweifeln an ihrer eigenen Kompetenz hinsichtlich einer Auseinandersetzung mit diesen Transformationen, was womöglich ebenfalls eine Hürde darstellt, ihnen im Unterricht zu begegnen. Solchen Annahmen soll dieser Beitrag entgegenwirken, indem er das Potenzial der Transformation von Franz Kafkas Die Verwandlung auslotet, um damit ein Urteil über die Axt-Kompetenz zumindest einer Adaption fällen zu können.

\section{Comics und Graphic Novels}

Im Grunde handelt es sich bei Comics und Graphic Novels um Formate moderner Bildgeschichten, die weder einem narrativen Einzelbild noch einem illustrierten Verbaltext entsprechen. Durch den Einsatz unbewegter, sich aufeinander beziehender Einzelbilder in Form eines Printmediums grenzt sich dieses Format ferner von Zeichentrickfilmen oder den neuesten digitalen Medien ab. Der Comic als Terminus schließt dabei in den meisten Fällen die spezifischen Spielarten Graphic Novel und Manga mit ein und besteht (zumeist) aus einem verbalen und piktoralen Zeichensystem, in deren funktionalem Zusammenspiel eine zentrale Eigenart dieses Formats $\mathrm{zu}$ finden ist (DolleWeinkauff, 2014). Obgleich diese Text-Bild-Kombinationen ein essenzielles Mittel der Narration darstellen, sind sie keineswegs ein obligatorisches; Charakteristisch für diese Formate ist die fakultative Möglichkeit dieser Interdependenzen (Palmier, 2015). Der sequentielle, an die Leserichtung der deutschen Schriftsprache orientierte Aufbau ist dabei gleichsam konstituierend wie die relativ unselbstständigen Einzelbilder (Dolle-Weinkauff, 2014).

Neben der Ausdifferenzierung zahlreicher Stoffe und Themen für den Comic sind auch neue Spielarten eingeführt worden, welche das Feld des Comics weiter ausbauen. Neben dem ,Manga' auch die seit den 80er-Jahren relative gebräuchliche ,Graphic Novel`. Dolle-Weinkauff zufolge beschreibt letztere jedoch nicht eine eigenständige Gattungsbezeichnung, sondern vielmehr einen literaturkritischen Wertungsbegriff, der literarisch und künstlerisch ,wertvollere' Bildgeschichten als solche hervorheben soll (ebd.). Auch Eders (2016) Auffassung ähnelt dieser. Letztendlich sprächen diese Argumente bei ihr

Kapelari, Suzanne (Hg.), Vierte „Tagung der Fachdidaktik“ 2019:

„Interdisziplinäre fachdidaktische Diskurse zur Bildung für nachhaltige Entwicklung“

(C) 2020 innsbruck university press, ISBN 978-3-99106-019-2, DOI 10.15203/99106-019-2 
allerdings für eine Genrebezeichnung, denn (bloß) für einen Wertungsbegriff. Die Uneinigkeit in der konkreten Klassifizierung findet sich auch in der Einund Abgrenzung dieses Mediums. Es handelt sich bei einer Graphic Novel nicht immer, wie die deutsche Übersetzung vermuten lässt, um einen Roman (oder eine romanhafte Erzählung) (Dolle-Weinkauff, 2012).

Der Begriff ,Graphic Novel' entstand in den 70er-Jahren, der Durchbruch gelang dem Begriff jedoch mit Will Eisner 1978, der sich mit seinem Werk $A$ Contract with God bewusst gegen die ,Profanität‘ des Comics wenden wollte, um aufzuzeigen, dass es sich bei seinem Werk um ein, wahres' Comicbuch handle, welches ,ernsthafte' Literatur an ein adoleszentes oder gar erwachsenes Publikum richte und zwar mit einer erzählerischen Tiefe, die Subtilität und Komplexität bietet (Eder, 2016.).

Merkmale zu finden, die für dieses Format konstituierend sind, gestaltet sich mitunter schwierig. Einige Charakteristika treffen auf Comics genauso zu, andere wiederum finden sich nicht in allen Graphic Novels wieder. Als Beispiel sei hier zunächst die serielle Erscheinungsform angeführt: Selten werden Graphic Novels in serieller Form publiziert (vgl. hierbei das Lustige Taschenbuch mit seiner mittlerweile 500. Ausgabe), doch existieren einige Graphic Novels, die sehr wohl als Serie verfasst und publiziert worden sind (vgl. Spiegelmanns Maus, Sartrapis Persepolis u.e.w.). Vielfach wird den Graphic Novels darüber hinaus ein literarischer Anspruch, den sie mitunter auch explizit erheben, zugeschrieben. Diese Zuweisung erheben allerdings auch eine Vielzahl von Comics (vgl. Das Urteil von Moritz Stetter, das er wiederum ganz bewusst nicht als eine Graphic Novel bezeichnete). Merkmale zu finden, die ausschließlich für Graphic Novels gelten, erschöpfen sich rasch in der hier kurz aufgezeigten verworrenen Beziehung zum Comic. Ähnlich verhält es sich mit der Themenwahl: Die gewählten Motive für Graphic Novels sind ebenso zahlreich wie die ästhetischen Innovationen, mit denen diese dargestellt werden können - auch dieser Umstand kann im gleichen Maße für Comics gelten. Es bleibt daher festzuhalten, dass nach wie vor keine klare Typologie der Graphic Novel existiert und es nur Ansätze einer Klassifikation (vgl. hierzu Baetens \& Frey, 2015) gibt (Eder, 2016.).

Kapelari, Suzanne (Hg.), Vierte „Tagung der Fachdidaktik“ 2019:

„Interdisziplinäre fachdidaktische Diskurse zur Bildung für nachhaltige Entwicklung“

(C) 2020 innsbruck university press, ISBN 978-3-99106-019-2, DOI 10.15203/99106-019-2 


\subsection{Literaturadaptionen in Form von Comics und Graphic Novels}

Schließlich ist es die Etablierung der Graphic Novels, mit der nicht bloß eine allmähliche Anerkennung der Formate insgesamt als Kunstform einhergeht, sondern die auch die Entwicklung der (wie Schmitz-Emans sie nennt) Literaturcomics impulsgebend mitverantwortet (Schmitz-Emans, 2016).

Literaturcomics bezeichnen jene Comics und Graphic Novels, „denen ein literarischer Text zugrunde liegt" (ebd.). Grundsätzlich unterscheiden sich diese Erscheinungsformen durch ihre Nähe zum Ausgangstext sowie durch die Motive für die Wahl der literarischen Vorlage. Dieser Prätext ist zumeist ein weltliterarischer Klassiker (Goethes Faust, Melvilles Moby Dick, Erzähltexte Kafkas u.v.a.), kann aber ebenso einem bestimmten Genre zugeordnet werden, wie die Vorliebe zur Adaption von Schauer- oder Fantasyliteratur (Strokers Dracula u.v.w.) beweist. Als Prätexte fungieren zumeist erzählende und dramatische Werke, während intermediale Übersetzungen von lyrischen Werken eher selten vorzufinden sind (ebd.). Solche Literaturadaptionen in Form von Comics und Graphic Novels sind letztlich das Ergebnis des Transfers eines literarischen Textes, welcher in das ,neue“ Medium übersetzt wird. Dadurch sind diese Transformationen nicht bloß intertextuell, weil sie auf die schriftlichen Bestandteile des Prätextes verweisen, sondern sie sind überdies intermedial, weil ,sie sich aus mindestens zwei verschiedenen medialen Quellen speisen“ (Blank, 2015). Diese Quellen sind verbaler und visueller Natur (hier: Text und Bild) und fallen laut Werner Wolf unter den Punkt der Plurimedialität. Das Wolf'sche System der intermedialen Relationen bietet überdies weitere intermediale Perspektiven auf Literaturadaptionen in Form von Comics und Graphic Novels. So wird die Literaturadaption selbst als intermediale Transposition bezeichnet und umfasst sowohl den Transfer von inhaltlichen als auch formalen Bestandteilen des literarischen Textes (Medium A) in den Comic oder die Graphic Novel (Medium B). Ebenso ist das Medienprodukt häufig durch intermediale Referenzen gekennzeichnet. Diese beschreiben die Übernahme von erzählerischen oder visuellen Mitteln anderer Medienprodukte wie Film oder Werke aus der bildenden Kunst (z.B. Montage, Kameraeinstellungen, Holzschnitt etc.) (Wolf, 2002). Bei Literaturcomics und

Kapelari, Suzanne (Hg.), Vierte „Tagung der Fachdidaktik“ 2019:

„Interdisziplinäre fachdidaktische Diskurse zur Bildung für nachhaltige Entwicklung“

(C) 2020 innsbruck university press, ISBN 978-3-99106-019-2, DOI 10.15203/99106-019-2 
-Graphic Novels treten zumeist alle drei Erscheinungsformen (Plurimedialität, intermediale Transposition, intermediale Referenz) zusammen auf.

\section{Analyse der Literaturadaption}

In der folgenden Analyse beziehe ich mich auf die Literaturadaption Die Verwandlung von Franz Kafka ${ }^{2}$ des französischen Szenaristen Eric Corbeyran sowie des britischen Zeichners Richard Horne. In den Fokus geraten dabei Elemente der Narration und Ästhetik von Comics und Graphic Novels, die insbesondere für Literaturadaptionen gelten, wobei stets auf den Prätext rekurriert wird. Eingeflochten werden Darstellungsmittel, Verfahren und Funktionen dieser Transformation, um das Phänomen der Literaturadaption zu erschließen. Die vorgestellten Kategorien geben einen Einblick in eine größere Analysearbeit und beziehen sich vornehmlich auf jene Module, die Blank 2015 für Literaturadaptionen im Comic vorstellte.

\subsection{Verhältnis zum Prätext}

Die Verwandlung ist nicht bloß eines der Werke Kafkas, mit dem sich Forschende am häufigsten auseinandergesetzt haben, der Großteil der zu Kafkas Oeuvre geführten Kontroversen, beschäftigt sich mit dieser Erzählung. Zahlreiche Deutungsaspekte sind bislang diskutiert worden, der Prätext dieser Graphic Novel bietet für sich betrachtet schon einen reichen Fundus an Interpretationsmöglichkeiten. Auch im schulischen Kontext genießt diese Erzählung kanonischen Status. Corbeyran und Horne haben sich für ihre Adaption demnach eines Textes angenommen, der den Status eines Klassikers innehat und sich damit in der typischen Riege jener intermedialen Übersetzer platziert, die sich mit Transformationen sogenannter ,Weltliteratur beschäftigen. Für dieses Kapitel sind nun jene Deutungsaspekte relevant, bei

2 La Métamorphose im Original. Übersetzung ins Deutsche von Kai Wilksen.

Kapelari, Suzanne (Hg.), Vierte „Tagung der Fachdidaktik“ 2019:

„Interdisziplinäre fachdidaktische Diskurse zur Bildung für nachhaltige Entwicklung“

(C) 2020 innsbruck university press, ISBN 978-3-99106-019-2, DOI 10.15203/99106-019-2 
denen zwischen der Adaption und dem Original die größte Diskrepanz vermutet werden kann.

Das Insekt selbst kann nicht gezeichnet werden. Es kann aber nicht einmal von der Ferne aus gezeigt werden. [...] Wenn ich für eine Illustration selbst Vorschläge machen dürfte, würde ich Szenen wählen, wie: die Eltern und der Prokurist vor der geschlossenen Tür oder noch besser die Eltern und die Schwester im beleuchteten Zimmer, während die Tür zum ganz finsteren Nebenzimmer offen steht (An G.H. Meyer [Kurt Wolff Verlag] 25.10. 1915; B14-17 145).

Kafka wehrte sich stets entschieden gegen eine Illustration des Ungeziefers, wohl um die Unbestimmtheit dieses Wesens bewahrt zu wissen - auch sprach er niemals von einem Käfer oder einer Schabe, sondern von einem Insekt oder Ungeziefer. Dadurch blieben dem Text Unbestimmtheitsgrade erhalten, die zu vielseitigen Interpretationen seitens der Forschung geführt haben (Poppe, 2010) unter anderem auch zu der Frage, ob die Verwandlung in ein Ungeziefer überhaupt stattgefunden hat oder der gesamte Prozess als metaphorisch gelesen werden kann (vgl. hierzu die Ausführungen Fingerhuts, 1994.)

Die Literaturadaption als Graphic Novel hingegen schlägt einen anderen Weg ein, indem sie bereits vor Beginn der Erzählung eine Schabe als Titelbild zeigt (Abb. 1.1) sowie als Einleitung eine Vielzahl von Informationen über das Insekt, die Schabe, die Kakerlake bietet und so das Rätsel um die Gestalt Samsas auflöst, um die absurde Gesamtsituation detailliert erfassen zu können (Blank 2011). 


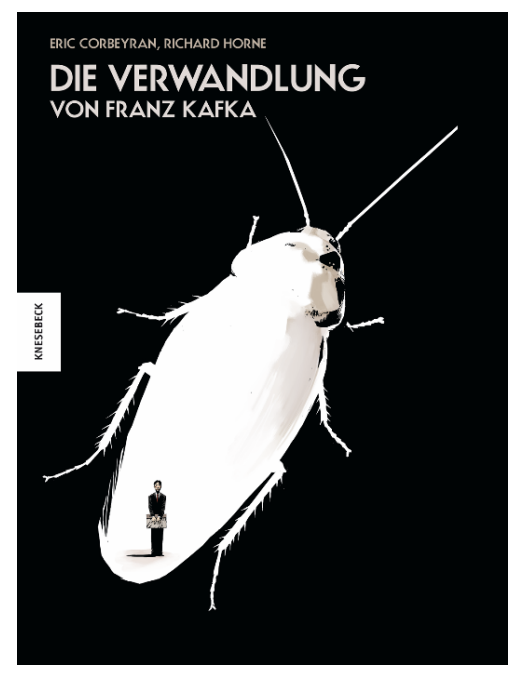

Abb. 1.1 Titelbild der Graphic Novel: Die Verwandlung von Franz Kafka

An dieser Stelle drängt sich nun die Frage auf, wie man diese Adaption noch weiterlesen soll, die sich derartig gegen den Wunsch des Autors wendet. Schließlich bezweckte Kafka mit diesem Verzicht eine gewisse Wirkung, die er beim Lesenden erzeugen wollte. Dass diese auf die Rezipierenden dieser Graphic Novel nun eine vollständig andere ist, wird kaum jemand bezweifeln können. Andererseits macht genau dieser Umstand die Literaturadaption in der Graphic Novel schlussendlich $\mathrm{zu}$ dem, was sie ist und was sie darstellen möchte: etwas Eigenständiges. Der Begriff der Werktreue ist für Adaptionen jedweder Art (sei es die Verfilmung oder der Literatur-Comic) längst überholt (Bohnenkamp, 2005). Eine große Nähe zum Prätext ist nicht automatisch literarisch ,wertvoller' oder, ernster zu nehmen“ als eine Abweichung. Comics und Graphic Novels arbeiten mit eigenen Darstellungsmitteln, die eine explizite Analyse verlangen und die sowohl auf bestehende Deutungen zurückgreifen, als eben auch, so wie beispielsweise durch die explizite Bebilderung des Ungeziefers, neue Aspekte hinzufügen. 
Inwiefern es der Graphic Novel nun weiters gelingt, zentrale Aspekte des Prätextes aufzugreifen und sie in Bild und Text zu übertragen, soll das folgende Beispiel aufzeigen. Trotz der Tragik, welche die Handlung zumeist durchzieht, ist Kafkas Text auf eigentümliche Art und Weise komisch, was unter anderem slapstickartig anmutet (Schmitz-Emans, 2010). So erscheinen die Gedanken Samsas bezüglich seiner bevorstehenden Arbeit und der Probleme aufgrund seiner Verspätung geradezu unsinnig und lustig, bedenkt man die Situation, in der er sich befindet und die im Grunde seine vollständige Aufmerksamkeit verdienen würde. Berechtigt stellt man hier die Frage, wie es der Graphic Novel gelingen kann, solche Elemente der Komik in Bild und Text aufzugreifen. Die Forschung sieht eine solche Möglichkeit der Übertragung durch die Verwendung sogenannter kontrapunktischer Bilder (Zöhrer, 2008). Dabei liefern Text und Bild völlig konträre Informationen zur Narration, wodurch Komik erzeugt werden kann. Die oben zitierte Szene wurde in der Adaption durch kontrapunktische Bild-Text-Kombinationen wiedergegeben. Samsas Gedanken drehen sich um den Chef und den erbarmungslosen Krankenkassenarzt, während die Bilder seinen ekelerregenden Körper zeigen. Die Diskrepanz zwischen dem Gezeigten und dem Beschriebenen soll auch hier Komik erzeugen. Offen bleibt, inwiefern diese ,komische" Intention der Autoren von den Lesenden rezipiert wird.

\subsection{Oberfläche}

Unterschieden wird hier grob zwischen der visuellen und der verbalen Oberfläche der Adaption. Bezüglich der verbalen Gestaltung interessiert vor allem, inwiefern sich die Autoren am Original orientierten oder in Abgrenzung dazu den Prätext modernisierten, abänderten etc. Corbeyran und Horne war offenbar sehr daran gelegen, Kafkas Erzählung textgetreu in das intermediale Format zu übersetzen, wie noch bei vielen anderen Analysekategorien deutlich wird. Ihrer Adaption haftet damit einhergehend etwas Anspruchsvolles an, da sie die Sprachgestalt des Prätextes weitestgehend (abgesehen von Verkürzungen und syntaktischen Angleichungen) bewahrt haben (Blank, 2015). 
Im Hinblick auf die visuelle Oberflächengestaltung sind Fragen der Farbgestaltung und Linienführung relevant (ebd.). Die Bilder selbst sind realistisch gezeichnet, wirken beinahe wie Photographien, wodurch das Geschehen auf den Rezipierenden unmittelbarer wirkt. Typische Oberflächenmerkmale von (klassischen) Comics, wie Onomatopoetika oder verschiedenartige Sprechblasenformen werden so eingesetzt, dass sie Narration und Ästhetik maßgeblich mittragen, was zu einer Darstellung führt, die insgesamt wirklichkeitsnaher wirkt und damit die Ernsthaftigkeit der Erzählung aber auch des gesamten Transformationsprozesses unterstreicht. Das Einfühlen in den Protagonisten wird mit dieser Art der Darstellung, welche die Lesenden nah an das Geschehen ,bindet', trotz seiner Gestalt, die mitunter ekelerregend ist, verstärkt. Die vorherrschende Farbe in der behandelten Literaturadaption ist schwarz, wodurch ein düsteres, zeitgleich aber auch trauriges Bild der Geschehnisse gezeichnet wird. Auch die Schattensetzung ist in dieser Transformation ein bewusstes Mittel der Narration. Die Gesichter der Figuren sind durch tiefe Schatten gekennzeichnet, wodurch die Beschwerlichkeit ihrer Lage hervorgehoben wird. Sie wirken hierdurch traurig, verzweifelt, mitunter auch müde.

Auch Rahmung und Anordnung der Panels können als Träger für Narration und Ästhetik fungieren. Die weiß gezeichneten, geraden Rahmen verleihen der Erzählung eine Unmittelbarkeit und weisen zeitgleich auf die Gegenwärtigkeit der Geschichte hin, da dem Panel-Rahmen die Funktion obliegt, die Erzählzeit zu bestimmen (Schmitz-Emans, 2012). Ein weiterer wichtiger Anhaltspunkt für die Narration ist die Anordnung der Panels. Die Familie selbst sowie der noch nicht verwandelte Gregor sind in geraden, sorgfältig angeordneten Panels zu sehen. Das Ungeziefer hingegen findet sich in schiefen Panels wieder, wodurch der Eindruck verstärkt wird, Samsas Leben sei vollkommen , aus den Fugen geraten'. Auch das störende Moment des Protagonisten wird durch die Panelanordnung deutlich. Die sogenannte Intrusion (Mälzer, 2015) beschreibt das Eindringen von Einzelbildern Samsas in jene Panels, in denen die Familie zu sehen ist - das Ungeziefer stört das normale Familienleben sowohl auf der verbalen als auch auf der visuellen Ebene. Von besonderem Interesse sind

Kapelari, Suzanne (Hg.), Vierte ,Tagung der Fachdidaktik“ 2019:

„Interdisziplinäre fachdidaktische Diskurse zur Bildung für nachhaltige Entwicklung“

(C) 2020 innsbruck university press, ISBN 978-3-99106-019-2, DOI 10.15203/99106-019-2 
überdies die Panels der letzten Seite. Die Familie selbst ist (wie üblich) in geraden Panels gezeichnet, bloß sind diese insgesamt nicht länger lotrecht im Vergleich zur Seite, was die Frage aufwirft, ob hier ein moralisches Urteil gefällt wurde. Die Familie ist zwar offenbar mit sich im Reinen, das tote Ungeziefer schließlich wie Müll entsorgt, doch die Szenerie erscheint bizarr, beinahe sarkastisch, schließlich ist keines der Familienmitglieder über den Verlust des Sohnes sonderlich traurig gestimmt und diesem Umstand wird durch die schiefe Anordnung der in sich geraden Panels Rechnung getragen. Auch das in der Forschung diskutierte sarkastische Moment dieser Szenerie wurde hier aufgegriffen (Poppe, 2010).

Ein für das Modul der Oberfläche ebenso zentrales Darstellungsmittel sind visuelle Leitmotive. Insbesondere die Farbe Rot stellt ein solches Leitmotiv dar und steht in dieser Adaption vor allem für Erotik und Begehren. Die ,Frau im Pelzmantel' als analeptischer panelübergreifender Verweis trägt rot, signalisiert für Samsa als Mensch etwas Begehrenswertes, gleichzeitig aber etwas Unerreichbares; auch als Ungeziefer bleibt die Frau für ihn eine unerfüllbare Wunschvorstellung (schließlich ist er nun als Ungeziefer überhaupt nicht mehr in der Lage, eine Beziehung mit einer ,menschlichen“ Frau einzugehen); die Äpfel, mit denen Samsa grob beworfen wird, sind ebenfalls tiefrot - der Apfel steht hier als Symbol für Versuchung, aber auch Vertreibung. Als Samsa überdies seine Schwester Violine spielen hört, will er sie für sich ganz alleine haben, will sich mit ihr in seinem Zimmer verschließen und sie dabei keineswegs mit den anderen teilen. Diese Begierde wird vor allem dadurch deutlich, dass sich die Farbe des Kleides von beige/braun in den vorangehenden und nachstehenden Panels zu weinrot verwandelt und Samsa an diesem Kleid zu seiner Schwester hochklettert. Auch mit der oben bereits beschriebenen Schlussszene geht ein gewisses Gefühl der Erotik einher. Die sich lasziv in einem purpurroten Kleid streckende Schwester, signalisiert damit ein Stück weit ihre, Geschlechtsreife‘. 


\subsection{Figurendarstellung}

In enger Verbindung zur Handlung steht die Darstellung der Figuren in einer Literaturadaption in Form von Comics und Graphic Novels. Die Festlegung auf eine bestimmte Gestaltung der Akteure ist bereits eine Interpretation, da sie den rein verbalen Text hier visuell konkretisiert (Blank, 2015).

Vergleicht man die Figuren der Graphic Novel von Corbeyran und Horne mit jenen von The Trial (dt. Der Process von Franz Kafka) von Montellier und Mairowitz, so fällt auf, dass sich letztere bei der Figurenzeichnung des Protagonisten stark an Kafka selbst anlehnten, wodurch eine vollkommen andere Interpretation zum Tragen kommt, als in der hier behandelten Literaturadaption von Die Verwandlung (Abb. 1.2-1.3) Nur in wenigen Panels ist Gregor in Menschengestalt zu sehen und hier lässt sich zwar eine gewisse Ähnlichkeit zu Kafka erkennen, doch in weitaus geringerem Ausmaß, sodass diese Adaption nicht automatisch in eine autobiographische Interpretation der Ereignisse überleitet und damit den Deutungshorizont möglicherweise etwas mehr offen lässt, als es Montellier und Mairowitz handhaben, die sich sogar direkt an einem Bild Kafkas (Abb. 1.3) orientierten.

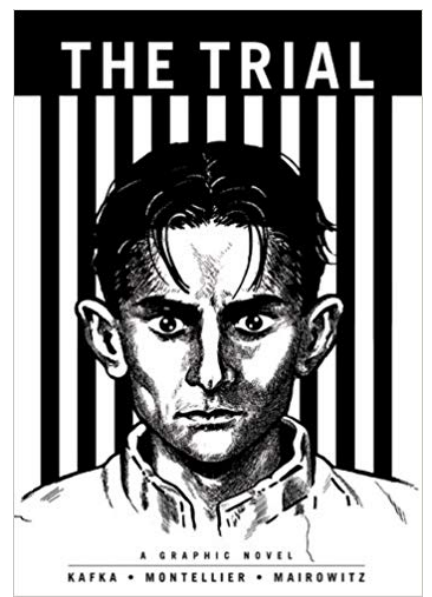

Abb. 1.2 Titelbild des Comics The Trial

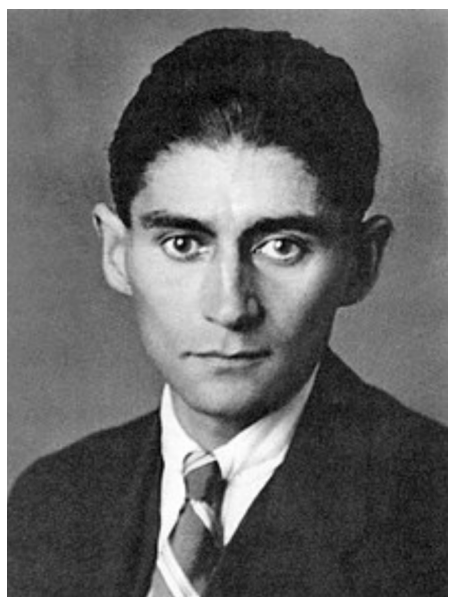

Abb. 1.3 Franz Kafka

Kapelari, Suzanne (Hg.), Vierte „Tagung der Fachdidaktik“ 2019:

„Interdisziplinäre fachdidaktische Diskurse zur Bildung für nachhaltige Entwicklung“

(C) 2020 innsbruck university press, ISBN 978-3-99106-019-2, DOI 10.15203/99106-019-2 
Wie sich Figuren mimisch und gestisch zeigen, kann ebenso Informationen für die Narration liefern. Während der Protagonist, ob seiner Verwandlung in eine Schabe, nur schwer bis gar nicht mimisch oder gestisch wahrnehmbar ist und in diesem Zusammenhang selbst mit seinem Menschenverstand wohl keine sichtbaren menschlichen Attribute mehr aufweist, sind die Gesichter der Familienmitglieder weitaus emotionaler gestaltet. Bis zum Tode Gregors hängen nicht bloß, wie oben bereits beschrieben, tiefe Schatten in den Gesichtern der Familie, sie sind überdies traurig, wütend und schockiert, beinahe jedoch nie fröhlich, solange Samsa noch am Leben ist, was die Tragik der Erzählung weiter verstärkt. Der Trübsinn, den die gesamte Familie ausstrahlt, wird ferner durch die Gestik greifbar. Zumeist sitzen sie gebückt, halten den Kopf gesenkt und wirken ausgelaugt und unverkennbar traurig. Einzig energisch bleibt zuweilen die Schwester, die ihre Wut häufig durch ausladende Gesten mit ihrer Hand zum Ausdruck bringen mag.

Besonders hervorzuheben sind außerdem die Formen der Sprechblasen. Während die Aussagen der übrigen Familienmitglieder in einer runden, abgeflachten Sprechblase platziert wurden, sind Gregors Sprechblasen unruhig und verzerrt. Diese Präzisierung des Prätextes auf visueller Ebene macht deutlich, dass Gregors Stimme sich bis ins Unverständliche verändert hatte:

Gregor erschrak, als er seine antwortende Stimme hörte, die wohl unverkennbar seine frühere war, in die sich aber, wie von unten her, ein nicht zu unterdrückendes, schmerzliches Piepsen mischte, das die Worte förmlich nur im ersten Augenblick in ihrer Deutlichkeit beließ, um sie im Nachklang derart zu zerstören, daß man nicht wußte, ob man recht gehört hatte (Die Verwandlung, 72).

Hierbei nimmt jedoch diese Sprechblasengestaltung der Graphic Novel möglicherweise etwas vorweg, was für den Text eine entscheidende Rolle spielt. Während Gregor nämlich seinen Monolog gegenüber dem Prokuristen hält und ihm versichert, bald wieder auf Reisen zu sein, weiß der/die Lesende des Prätextes noch nicht mit Sicherheit, dass er von Menschen nicht mehr 
verstanden wird, dies löst sich erst am Ende des Monologes auf, als der Prokurist die Flucht ergreift. Durch die Gestaltung der Sprechblasen, die sich doch deutlich von den übrigen Figuren unterscheidet, wird jedoch im Vorfeld schon ersichtlich, dass sich Gregors Stimme verändert hat und nicht mehr menschlich - und damit womöglich nicht länger verständlich - ist.

\subsection{Perspektive}

Wie diese Figuren nun im Raum wahrgenommen werden, ist eine Frage der Perspektive. Obgleich sich dieser Analysepunkt aus mehreren einzelnen Faktoren zusammensetzt, die einer Betrachtung wert wären, ist für diesen Beitrag die Fokalisierung ausschlaggebend. Laut Genette unterscheidet man drei Arten einer möglichen Perspektivierung: die Nullfokalisierung, die das Geschehen ohne perspektivische Einschränkung wiedergibt und somit am ehesten Stanzels auktorialer Erzählsituation entspricht, die interne Fokalisierung (Stanzel spricht hier von der personalen Erzählperspektive), welche nur die Sicht des Protagonisten zeigt, somit zwar Einblick in die Gefühlswelt dieser Figur bieten kann, nicht jedoch alles, was um diese Figur herum geschieht, adäquat erfasst und schlussendlich die externe Fokalisierung, bei der die Sicht auf die Handlung der Erzählung wiedergegeben wird, man jedoch nicht Einblick in die Gefühlswelt der Figuren erhält, was Stanzels neutraler Erzählperspektive am ehesten gleichzusetzen ist (Genette, 1994). Kafkas Die Verwandlung ist weitestgehend (bis auf den Schluss) intern fokalisiert. Die Lesenden sehen das Geschehen aus Gregors Augen und wissen daher nur, was auch Gregor weiß. Das Konzept der internen Fokalisierung lässt sich jedoch nicht ohne weiteres auf den Comic übertragen, schließlich ist es schwierig, einem Comic-Panel tatsächliches Wissen zu unterstellen. Daher fragt man bei Comics und Graphic Novels nach dem ,Wer sieht` nicht auf der verbalen, sondern auf der rein visuellen Ebene (Blank, 2015). Die Literaturadaption von Corbeyran und Horne arbeitet, um es mit Schüwer (2008) auszudrücken, durchaus mit einem halbsubjektiven Bild, d.h. wir sehen dem Protagonisten über die Schulter (in der Filmsprache der, over-the-shoulder-shot ${ }^{`}$ ) und erleben

Kapelari, Suzanne (Hg.), Vierte „Tagung der Fachdidaktik“ 2019:

„Interdisziplinäre fachdidaktische Diskurse zur Bildung für nachhaltige Entwicklung“

(C) 2020 innsbruck university press, ISBN 978-3-99106-019-2, DOI 10.15203/99106-019-2 
dadurch, wie der Protagonist das Geschehen wahrnimmt, sehen (und lesen) die Erzählung aus seinen Augen. Das halbsubjektive Bild ist daher am ehesten mit Genettes interner Fokalisierung gleichzusetzen. Die beiden Abbildungen zeigen die Perspektive Gregors, zumeist die filmtypische Froschperspektive, was einerseits durch die geringe Größe des Ungeziefers erklärbar ist, andererseits auch den Eindruck des Parasiten in der Familie verstärkt, wie er, klein und unnütz geworden, zur Familie aufblicken muss.

Häufig schwenkt das Panel in die extreme Außensicht - bei Genette die Nullfokalisierung - und fokussiert dabei die Familie und ihre Erlebnisse mit dem verwandelten Sohn oder das Insekt selbst in seinem „Menschenzimmer“ (zumeist aus verzerrter Perspektive). Damit wird ersichtlich, wie ,verrückt ${ }^{6}$ das Geschehen ist. Ohne erkennbaren Beobachter wird das verwandelte Insekt gezeigt, sein Zimmer ist schief, seine Fühler überlang, die Decke des Raumes nicht ersichtlich, das Groteske der Verwandlung wird durch die Perspektive besonders betont. Obgleich der Prätext aufgrund seiner rein internen Fokalisierung diese Sicht auf das Geschehen nicht einnehmen kann, so bietet er doch Potenzial für diese Art der Darstellung. Der (berühmte) erste Satz der Erzählung lautet nämlich: „Als Gregor Samsa eines Morgens aus unruhigen Träumen erwachte, fand er sich in seinem Bett zu einem ungeheuren Ungeziefer verwandelt" (Die Verwandlung, 69). Durch die durchwegs eigenartige Formulierung „fand er sich“ ist auch diese Art der bildlichen Darstellung nicht ganz von der Hand zu weisen. Wie eine außerkörperliche Erfahrung „fand“ Gregor sich verwandelt vor.

Ein anderer Blick von außen befindet sich außerhalb der diegetischen Welt der Adaption auf der Innenseite des Einbandes. Den Rezipierenden wird schon vor der Lektüre die Gestalt Gregors mitgeteilt, eine Information, die aus Gregors Sicht nicht möglich ist und die auch bei Kafka selbst stets ein Rätsel geblieben ist. D.h. die Lesenden wissen bereits zu Beginn der Erzählung, mehr ‘̈ über die Verwandlung als der Protagonist, was bei der rein internen Fokalisierung und dem expliziten Verzicht auf Illustrationen im Prätext nicht möglich ist. Des Weiteren ist auch dann eine Nullfokalisierung gegeben, wenn Ausschnitte aus

Kapelari, Suzanne (Hg.), Vierte „Tagung der Fachdidaktik“ 2019:

„Interdisziplinäre fachdidaktische Diskurse zur Bildung für nachhaltige Entwicklung“

(C) 2020 innsbruck university press, ISBN 978-3-99106-019-2, DOI 10.15203/99106-019-2 
Prag gezeigt werden. Diese dienen einerseits dazu, das Geschehen zu kontextualisieren (Blank, 2015), also in Prag zu verorten, andererseits auch dazu, die von Kafka vorgegebene Einteilung in drei Kapitel deutlich zu machen. Das zweite Kapitel beginnt mit der Sicht auf die Dächer Prags, in einer Nacht, der Mond scheint nur hie und da durch die dichte Wolkendecke hindurch. Mit Beginn des dritten Kapitels ist ein anderer Stadtteil Prags zu sehen und die Dämmerung hat bereits eingesetzt, der Himmel ist rötlich-orange, im rechten Eck kann sogar das Licht der Sonne entdeckt werden. Die letzten Seiten sind schlussendlich bei Tageslicht dargestellt, vor allem als Gregor stirbt, ist die Nacht ,endgültig' vorüber und mit ihr das Problem der übrigen Familie Samsa, die glücklich in der Straßenbahn sitzt. Die durchbrochene Nacht bringt auch (endlich) wieder Sonne und damit Freude in das Leben der übrigen Familie Samsa.

Ist der Blick auf die übrigen Familienmitglieder gerichtet und Gregor kann als Betrachter ausgeschlossen werden, spricht man laut Genette von externer Fokalisierung. Dies dient unter anderem dazu, das Verhalten und die Gefühle der übrigen Figuren $\mathrm{zu}$ registrieren. Die Bilder zeigen, dass hier nicht ,nullfokalisiert` wird, sondern vielmehr aus Sicht eines Beobachters gezeigt wird, der sich nicht sichtbar im Raum mit den übrigen Figuren aufhält und so den Rezipierenden einen Blick auf die Familie bietet, der sich dem Protagonisten verschließt. 


\section{Zur geplanten empirischen Studie und deren didaktischem Horizont}

Da der Fokus dieses Beitrags vermehrt auf literaturwissenschaftlicher Analyse lag, könnte die Frage aufkommen, worauf ich damit in didaktischer Hinsicht verweisen möchte. Da in empirischer Hinsicht dem Comic und der Graphic Novel noch verhältnismäßig wenig Beachtung geschenkt wurde, möchte ich mit meiner qualitativen Studie diesem Missstand entgegentreten. Ob und inwiefern sich nämlich die Rezeption von Literaturadaptionen und deren Prätexten unterscheidet, und wie Phänomene der Narration in den jeweiligen Medien wahrgenommen werden, wird diese Studie offen legen. Mithilfe der Methode des Lauten Denkens rezipiert eine Gruppe von SchülerInnen der Sekundarstufe II einen Ausschnitt aus dem Erzähltext Kafkas, die andere Gruppe einen Ausschnitt aus der Adaption. Die daraus abzuleitenden Erkenntnisse sollen Aufschluss darüber geben, wie die beiden rezipiert werden und wie ein entsprechend informierter Unterricht auf Hilfen beim jeweiligen Textverständnis reagieren kann. Wie kann das eine Medium das Verständnis des anderen erhellen und vice versa? Wie kann durch den Einsatz des jeweiligen Mediums die Lesemotivation gesteigert werden? Dabei steht nicht bloß der Vergleich im Mittelpunkt der Analyse, sondern die relativ offen formulierte Frage, wie gewisse literarische Phänomene, die auch dieser Beitrag ausgelotet hat, in den jeweiligen Medien wahrgenommen werden. Das Ziel der Studie ist es nicht, eine ,bessere' Herangehensweise zu Literatur zu finden, sondern eine gegenseitige Ergänzung und Befruchtung $\mathrm{zu}$ finden - eine , $\mathrm{Axt}^{6}$, die durch verschiedene Verwendungsmöglichkeiten einen nachhaltigen Literaturunterricht erzielt. 


\section{Resümee}

Nachhaltiges literarisches Lernen kommt nicht an der sich stetig ändernden außerschulischen literarischen Wirklichkeit vorbei. Comics und Graphic Novels sind bereits in vielerlei Hinsicht Teil der literarischen Kultur. Der Buchhandel und die Bibliothek bieten eigene Abteilungen an, viele Buchmessen integrieren diese Art der Literatur bereitwillig in ihr Programm. Der Literaturunterricht tut sich bislang jedoch schwer damit, einen sinnvollen Zugang zu diesen Medienformaten zu finden, was auch an den Lehrbüchern liegt, die Comics und Graphic Novels nur marginal bis gar nicht aufgreifen. Nichtsdestotrotz sollte hier freilich nicht ein blindes Aufnehmen sämtlicher Adaptionen in den Vordergrund gerückt werden, sondern vielmehr ist es mir ein Anliegen, die vielseitigen Möglichkeiten aufzuzeigen, wie man in den Genuss von Literatur kommen kann und wie fruchtbar sich eine Analyse und damit einhergehend eine Deutung gestalten kann.

Mit eigenen Darstellungsmitteln und zahlreichen Möglichkeiten, Narration und Ästhetik mithilfe von Text und Bild zu gestalten, gelingt es vielen Comics und Graphic Novels, Prätexte geschickt zu adaptieren. Dies führt mitunter auch zur unangebrachten Hoffnung, solche Adaptionen würden die Lesenden , an die Originalwerke" heranführen, wofür jedoch keine empirischen Beweise vorliegen. Darüber hinaus gilt, unterhaltende" Literatur dann als ,fertig gelesen“, wenn die Handlung weitestgehend nachvollzogen wurde. Die Ansicht, eine Literaturadaption könnte als „Einstiegsmedium“ (Blank, 2015) fungieren, kann in Bezug auf diese Transformation nicht gelten, schließlich kann die Handlung von Die Verwandlung durch die Adaption gut verstanden werden. Es bleibt der Appell, Literaturadaptionen in Form von Comics und Graphic Novels als eigenständige Kunstwerke zu betrachten, die weder dem Prätext genauestens entsprechen, noch als sein Sprungbett fungieren müssen.

Bildung für nachhaltige Entwicklung bedeutet für den Literaturunterricht einerseits das Aufgreifen aktueller Themen wie Toleranz, Offenheit, Akzeptanz u.v.w. Andererseits kann Literaturunterricht auch auf einer tiefergreifenden Ebene für Nachhaltigkeit sorgen, indem die außerschulische literarische Realität in den Unterricht integriert wird, um so ein nachhaltiges literarisches Lernen zu

Kapelari, Suzanne (Hg.), Vierte ,Tagung der Fachdidaktik“ 2019:

„Interdisziplinäre fachdidaktische Diskurse zur Bildung für nachhaltige Entwicklung“

(C) 2020 innsbruck university press, ISBN 978-3-99106-019-2, DOI 10.15203/99106-019-2 
ermöglichen. Diese Form der Nachhaltigkeit bezieht sich dabei eben nicht auf explizite Themen der BNE, sondern auf das Herstellen einer inneren, bewussten Form der Nachhaltigkeit, die sich durch intensive, subjektorientierte Lektüre definiert und damit schlussendlich Nachhaltigkeit herstellt.

\section{Literatur}

Primärliteratur

Kafka, F. (2017). Die Verwandlung. In M. Müller (Eds.). Franz Kafka. Erzählungen (pp. 67-132). Stuttgart: Reclam. (=Universalbibliothek, 9426).

Corbeyran, E., Horne, R. (2010). Die Verwandlung. Von Franz Kafka. Übersetz. von Kai Wilksen. München: Knesebeck.

Sekundärliteratur

Baetens J., Frey, H. (2015). The Graphic Novel. An Introduction. Cambridge: Cambridge University Press.

Blank, J. (2015). Literaturadaptionen im Comic. Ein modulares Analysemodell. In M. Schmitz-Emans \& D. Grünewald (Eds.), Bildnarrative, Studien zu Comics in Bilderzählungen. Berlin: Bachmann.

Blank, J. (2011). Erzählperspektive im Medienwechsel. Visuelle Fokalisierung in Comic-Adaptionen von Texten Franz Kafkas. https://edoc.huberlin.de/bitstream/handle/18452/8106/blank.pdf?sequence=1\&isAllowed=y

Bohnenkamp, A. (2005). Literaturverfilmungen als intermediale Herausforderung. Ein hybrides Genre. In A. Bohnenkamp (Eds.), Literaturverfilmungen (pp. 9-40). Stuttgart: Reclam. (=Universal-Bibliothek, 17536).

Dolle-Weinkauff, B. (2014). Comic, Manga, Graphic Novel. In A. Tillmann et al. (Eds.), Handbuch Kinder und Medien (pp. 457-468). Wiesbaden: Springer Fachmedien. (=Digitale Kultur und Kommunikation, 1).

Eder, B. (2016). Graphic Novels. In J. Abel \& C. Klein (Eds.), Comics und Graphic Novels. Eine Einführung (pp. 156-168). Stuttgart: Metzler.

Fingerhut, K. (1994). Die Verwandlung. In M. Müller (Eds.), Interpretationen. Franz Kafka: Romane und Erzählungen (pp. 42-74). Stuttgart: Reclam. (=UniversalBibliothek 17521).

Genette, G. (1994). Die Erzählung. Übersetz. v. Andreas Knop. München: UTB. (=UniTaschenbücher, 8083). 
Kafka, F. (1966). Briefe 1902-1924. In M. Brod (Eds.). Frankfurt a.M.: Fischer.

Mälzer, N. (2015). Taxonomien von Bild-Text-Beziehungen im Comic. In N. Mälzer (Eds.), Comics - Übersetzungen und Adaptionen (pp. 47-64). Berlin: Frank \& Timme.

Montellier, C.; Mairowitz, R. Z. (2008). The Trial. A Graphic Novel. New York, London: Sparknotes.

Palmier, J.P. (2015). Strukturverwandte. Zur Adaptierbarkeit von Erzählungen in Literatur, Comic und Film. In N. Mälzer (Eds.), Comics - Übersetzungen und Adaptionen (pp. 81-96). Berlin: Frank \& Timme.

Poppe, S. (2010). Die Verwandlung. In M. Engel \& B. Auerochs (Eds.), KafkaHandbuch. Leben - Werk - Wirkung (pp. 164-174). Stuttgart, Weimar: J.B. Metzler.

Schmitz-Emans, M. (2016). Literaturcomics. In J. Abel \& C. Klein: Comics und Graphic Novels. Eine Einführung (pp. 276-290). Stuttgart: Metzler.

Schmitz-Emans, M. (2012). Literatur-Comics. Adaptationen und Transformationen der Weltiteratur. Berlin. Walter de Gruyter.

Schmitz-Emans, M. (2010). Franz Kafka. Epoche - Werk-Wirkung. In W. Barner \& G. E. Grimm (Eds.). München: C.H.Beck.

Schüwer, M. (2008). Wie Comics erzählen. Grundriss einer intermedialen Erzähltheorie der grafischen Literatur. Trier: WVT.

Stanzel, F. K. (1993). Typische Formen des Romans. Göttingen: Vandenhoeck u. Ruprecht.

Wolf, W. (2002). Intermediality Revisited. Reflections on Word and Music Relations in the Context of a General Typology of Intermediality. In S. M. Lodato et al. (Eds.), Word and Music Studies. Essays in Honor of Steven Paul Scher and on Cultural Identity and the Musical Stage (pp. 13-34). Amsterdam: Rodopi.

Zöhrer, M. (2008). Weltliteratur im Bilderbuch. In E. Seibert und H. Lexe (Eds). Wien: praesens.

Abbildungsverzeichnis

Abb. 1.1 Corbeyran, Eric; Horne, Richard (2010). Die Verwandlung. Von Franz Kafka. Übersetz. von Kai Wilksen. München: Knesebeck.

Abb. 1.2 Montellier, Chantal; MAIRowitz, Robert Z. (2008): The Trial. A Graphic Novel. New York, London: Sparknotes.

Abb. 1.3; Porträt Franz Kafkas aus dem Jahre 1923. URL:

https://de.wikipedia.org/wiki/Franz Kafka [zuletzt aufgerufen am 10. April 2019].

Kapelari, Suzanne (Hg.), Vierte ,Tagung der Fachdidaktik“ 2019:

„Interdisziplinäre fachdidaktische Diskurse zur Bildung für nachhaltige Entwicklung“

(C) 2020 innsbruck university press, ISBN 978-3-99106-019-2, DOI 10.15203/99106-019-2 



\title{
Implizite Bedeutung in sozialen Netzwerken Stärkung des gesellschaftlich-demokratischen Bewusst- seins im Französisch als Fremdspracheunterricht
}

\author{
Dr. Nicola Brocca, Universität Innsbruck
}

\section{Einleitung}

Am Anfang der 2010er Jahre habe ich in einer Gemeinschaftsschule in BerlinKreuzberg Französisch unterrichtet. Smartphones verschafften sich in dieser Zeit den Weg in die Tasche der SchülerInnen und aus den Schulranzen piepste es während des Unterrichts immer häufiger. Da die Smartphones nicht mehr mit Verboten einzudämmen waren, beschlossen wir Lehrkräfte ihre Nutzung zumindest zu regulieren. Bei einer Schulkonferenz entschied das Kollegium einen Raum für freie Smartphonenutzung zu schaffen und die Anwendung von internetfähigen Endgeräten bei Bedarf im Unterricht $\mathrm{zu}$ erlauben. Die Smartphone Ecke wurde von SchülerInnen sehr positiv begrüßt: In den Pausen versammelten sich viele SchülerInnen dort auf einigen Sitzsteinen im Schulhof. Einige SchülerInnen spielten ,Grand Theft Auto' und holten sich auf YouTube Tipps, um sich zu verbessern. Andere nahmen Videoclips mit musical.ly auf und teilten sie auf Facebook. Jedoch blieb manchmal das, was die SchülerInnen mit ihren Geräten machten, uns Lehrkräften verborgen. Dies änderte sich, als einige Wochen später wiederholt Meldungen bezüglich Konflikten und sogar Straftaten, die auf den ungeregelten und unkritischen Umgang mit Social Network Sites (SNSs) zurückzuführen waren, unsere Schule erreichten. Seitdem

Kapelari, Suzanne (Hg.), Vierte ,Tagung der Fachdidaktik“ 2019:

„Interdisziplinäre fachdidaktische Diskurse zur Bildung für nachhaltige Entwicklung“

(C) 2020 innsbruck university press, ISBN 978-3-99106-019-2, DOI 10.15203/99106-019-2 
wurde die handyfreie Zone abgeschafft und ein gänzliches Verbot von Mobiltelefonen wiedereingeführt. Damit spielte sich die Mediennutzung nicht mehr unter den Augen der Lehrkräfte ab, und die schulische Bildung legte ihren Einfluss auf was die SchülerInnen in SNSs lasen, lernten und weiterleiteten ab.

Die Reaktion dieser Berliner Schule war kein Einzelfall; allgemein zeigen sich Schulen sehr zurückhaltend gegenüber internetfähigen Mobilgeräten: Laut der JIM-Studie (2016) darf nur ein Drittel der befragten deutschen SchülerInnen das Handy in der Schule ausschließlich in den Pausen nutzen. Die Tendenz, internetfähige mobile Endgeräte im Unterricht einzusetzen, ist jedoch rasch gestiegen. 2015 durften nur 7\% der SchülerInnen ihr Handy gezielt im Schulunterricht einsetzen. 2016 belief sich dieser Anteil bereits auf 22\% (JIMStudie, 2015, 2016). Besser erscheint die Haltung gegenüber Smartphones oder Tablets in Österreich: Nach Angabe des Bundesministeriums für Bildung, Wissenschaft und Forschung (BBWF) wird an rund zwei Dritteln der NMS, AHS und BMHS ${ }^{1}$ mit eigenen Geräten (Smartphones, Tablets oder Notebooks) bei Bedarf im Unterricht gearbeitet (Bundesministerium für Bildung Wissenschaft und Forschung, 2019, S. 1). Die Praxis außerhalb der Schule sieht demgegenüber jedoch anders aus: Über drei Stunden verbringen deutsche Jugendliche durchschnittlich jeden Tag nur mit SNS (DAK-Gesundheit \& UKE, 2018; JIM, 2017). Neben der Befriedigung von sozialen Bedürfnissen ist das Interesse für aktuelle politische Themen etwas, das die Jugendlichen in SNS bewegt (JIM, 2017): 34\% der 18-19-Jährigen geben an, primär in Facebook Nachrichten zum aktuellen Weltgeschehen zu suchen (ebd.).

Das zeigt, dass SNS sich als Online-Nachrichtenmedien etabliert haben. Die Auswirkungen dieser Entwicklung sind noch nicht vollständig verstanden worden, insbesondere wenn es um die Rolle der SNS bei der Gestaltung der Überzeugungen der Menschen in einem zunehmend polarisierten Europa geht. SNS sind für PolitikerInnen von besonderem Wert, da sie direkter und in allgegenwärtigen Situationen mit ihren AnhängerInnen kommunizieren können als zuvor.

1 NMS steht für Neue Mittelschule, AHS für allgemein bildende höhere Schule und BMHS für Berufsbildende mittlere und höhere Schule.

Kapelari, Suzanne (Hg.), Vierte „Tagung der Fachdidaktik“ 2019:

„Interdisziplinäre fachdidaktische Diskurse zur Bildung für nachhaltige Entwicklung“

(C) 2020 innsbruck university press, ISBN 978-3-99106-019-2, DOI 10.15203/99106-019-2 
Das Verstehen von politischen Botschaften in SNS und die kritische Auseinandersetzung mit ihnen kann als übergreifende Kompetenz für die Realisierung der Ziele für die Nachhaltige Entwicklung (SDGs) gesehen werden. Dabei findet sich diese Kompetenz in erster Linie im Ziel der hochwertigen Bildung (Ziel 4 SDGs). Darüberhinausgehend kann sie auch der Förderung von friedlichen und gerechten Institutionen (Ziel 16 SDGs) und inklusiven Gesellschaften (Ziel 10 SDGs) zugeordnet werden (siehe Rieckmann in diesem Band).

Die SDGs und die auf ihnen basierenden Ziele der Agenda 2030 (Vereinte Nationen, 2015) überlassen den nationalen Staaten die Umsetzung der Ziele. Da in Europa demokratische Institutionen sowie der gesellschaftliche Zusammenhalt in den letzten Jahren immer wieder gefährdet $\operatorname{sind}^{2}$, sollte ihre Verstärkung und ihr Schutz auch im Rahmen der SDGs von prioritärer Wichtigkeit werden.

Eine Bildungsstrategie, die die SchülerInnen im Sinne der Ziele der SDGs bilden möchte, muss die Förderung der Demokratie und einer integrativen und kohäsiven Gesellschaft berücksichtigen. Dazu gehört auch die Kompetenz jüngerer Menschen, in der Lage zu sein, sich aus Fakten ein Meinungsbild zu verschaffen und Manipulationsversuche zu erkennen. Wenn sich junge Menschen immer öfter auf Basis von sozialen Medien ihre Meinung bilden, kann das Bildungssystem sich zukünftigen Herausforderungen der Kommunikation online nicht verschließen. Das Strategiepapier der ständigen Konferenz der Kultusminister der Länder in der Bundesrepublik Deutschland zur „Bildung in der digitalen Welt“ (KMK, 2017) sieht vor, den Bildungserfolg noch stärker darauf auszurichten, „Fakten, Prozesse, Entwicklungen einerseits einzuordnen und zu verknüpfen, andererseits zu bewerten und dazu Stellung zu nehmen“ (S.13). Hierzu kann eine bewusste und kritische Anwendung von Internetressourcen einen wesentlichen Beitrag leisten. Auch im österreichischen bildungspolitischen Diskurs wird die Verschränkung zwischen Informations-

2 Z.B. der Aufstieg von EU- und minderheitsfeindlichen Parteien oder der Brexit.

Kapelari, Suzanne (Hg.), Vierte „Tagung der Fachdidaktik“ 2019:

„Interdisziplinäre fachdidaktische Diskurse zur Bildung für nachhaltige Entwicklung“

(C) 2020 innsbruck university press, ISBN 978-3-99106-019-2, DOI 10.15203/99106-019-2 
kompetenzen und sozial-politischem Zusammenhalt betont: Der ,produktive Umgang mit Medien für eine kritische, bewusste [...] staatsbürgerliche Teilhabe“ (S. 97) wird als Ziel der Medienerziehung im Nationalen Bildungsbericht Österreich definiert (Baumgartner, Brandhofer, Ebner, Gradinger \& Korte, 2015).

Dieser Aufsatz beschäftigt sich mit der Frage, welchen Beitrag die Sprachwissenschaft und die Fremdsprachendidaktik leisten können, um Ziele in der Medienbildung zu verfolgen, die eine demokratische und integrative Gesellschaft fördern. Dabei wird auf theoretische Erkenntnisse der Grice'schen Pragmalinguistik (Grice, 1975) zurückgegriffen und daraus eine Lerneinheit mit Content and Language Integrated Learning (CLIL) Ansatz skizziert. Dieser Beitrag argumentiert, warum - trotz gewisser Hürden - die Fähigkeit, soziale Netzwerke kritisch zu lesen, eine grundlegende Kompetenz für eine Bildung, die die SDG fördert, darstellt und warum es daher sinnvoll ist, diese Kompetenzen (auch) im Fremdsprachenunterricht zu vermitteln. Der Beitrag ist folgendermaßen aufgebaut: In Abschnitt zwei wird die Präsenz der kritischen Medienbildung in Lehrplänen untersucht. Abschnitt drei behandelt die Rolle der Pragmalinguistik als Mittel des kritischen Textverständnisses, während der vierte Abschnitt die medialen und sprachlichen Kompetenzen zusammenführt. Abschießend wird in Abschnitt fünf eine Zwischenbilanz zur Validität und Durchführbarkeit der vorgestellten Materialien gezogen. Im Anhang finden sich Unterrichtsmaterialien, die exemplarisch zeigen sollen, wie diese Überlegungen in die Praxis des Fremdsprachunterrichts übertragen werden können.

\section{Medienbildung und gesellschaftliche Teilhabe im Lehrplan für Fremdsprachen}

$\mathrm{Zu}$ den Zielen der Vereinten Nationen für eine Nachhaltige Entwicklung zählt die Förderung friedlicher und integrativer Gesellschaften und der Aufbau effektiver, rechenschaftspflichtiger und integrativer Institutionen (Ziel 16 SDGs). $\mathrm{Zu}$ diesem Ziel gehören die uneingeschränkte gesellschaftliche 
Teilhabe, die Entwicklung effektiver, rechenschaftspflichtiger und transparenter Institutionen (Ziel 16.6 SDGs) sowie die Sicherstellung einer reaktionsschnellen, integrativen, partizipativen und repräsentativen Entscheidungsfindung (Ziel 16.7 SDGs) (Vereinte Nationen, 2015; siehe auch Rieckmann in diesem Band).

Ein Echo dieser Einstellung ist auch in den Lehrplänen der österreichischen allgemeinbildenden höheren Schulen zu finden: Zu den Aufgabenbereichen des schulischen Fremdsprachenunterrichts gehört demnach, das

wertorientierte Denken und Handeln im politischen, sozialen, wirtschaftlichen, kulturellen und weltanschaulichen Umfeld zu fördern. [...] Der Erwerb einer Fremdsprache dient u.a. [...] der bewussten Auseinandersetzung mit [...] Wahrnehmungen und Wertungen [und soll] zum [...] friedlichen Zusammenleben beitragen. (Bundesministerium für Unterricht und Kunst, 1985: Fassung vom 01.09.2018) (Hervorhebungen durch den Verfasser)

Die Lehrpläne nehmen keine Einschränkung der Medienarten vor, die in den jeweiligen Fächern zum Erreichen der Lernziele eingesetzt werden können:

das selbstständige Erschließen und Erfassen schriftlicher fremdsprachlicher Texte verschiedener Art mit Hilfe angemessener Lesestrategien [...] die produktive schriftliche Anwendung der erworbenen Sprachmittel in adressatenadäquater und mediengerechter, d.h. der jeweiligen Textsorte entsprechender, Form. (Bundesministerium für Unterricht und Kunst, 1985: Fassung vom 01.09.2018) (Hervorhebungen durch den Verfasser)

Wie in anderen EU Ländern (Brocca, Borowiec, \& Masia, 2020: S.83) wird die Einführung neuer Technologien als didaktische Medien explizit aufgrund der anzustrebenden Aktualität und Anschaulichkeit empfohlen:

Kapelari, Suzanne (Hg.), Vierte „Tagung der Fachdidaktik“ 2019:

„Interdisziplinäre fachdidaktische Diskurse zur Bildung für nachhaltige Entwicklung“

(C) 2020 innsbruck university press, ISBN 978-3-99106-019-2, DOI 10.15203/99106-019-2 
Die Materialien und Medien, die im Unterricht eingesetzt werden, haben möglichst aktuell und anschaulich zu sein, um die Schülerinnen und Schüler zu aktiver Mitarbeit anzuregen [...]. Den neuen Technologien kommt verstärkt Bedeutung zu. (Herstellen von Bezügen zur Lebenswelt). (Bundesministerium für Unterricht und Kunst, 1985: Fassung vom 01.09.2018)

Zur Arbeit mit neuen Technologien wird nicht nur geraten, um rezeptive Kompetenzen $\mathrm{zu}$ fördern, sondern auch, um produktive Kompetenzen und Lernautonomie zu stimulieren: „Die Erstellung eigenständiger Arbeiten mit Mitteln der Informationstechnologie ist anzuregen“ (Bundesministerium für Unterricht und Kunst, 1985 Fassung vom 01.09.2018). Die Einführung von digitalen Medien beschränkt sich nicht auf deren Rolle als didaktische Mittel. Laut Desiderata des österreichischen Bundesministeriums für Unterricht und Kunst können digitale Medien (und somit auch SNS) selbst Thema des Unterrichts werden, wie im folgenden Zitat deutlich wird:

Zur Erlangung eines möglichst umfassenden lexikalischen Repertoires (Handlungsfähigkeit/ Kommunikationsfähigkeit) sind verschiedenste Themenbereiche zu bearbeiten [...]; Rolle der Medien; Einstellungen und Werte; Zusammenleben; aktuelle soziale, wirtschaftliche, technische und politische Entwicklungen. (Bundesministerium für Unterricht und Kunst, 1985: Fassung vom 01.09.2018) (Hervorhebungen durch den Verfasser)

Obwohl die Förderung der Nutzung von digitalen Medien und OnlineInformationsdiensten im Unterricht an zahlreichen Stellen betont wird, bleiben die Ziele, die Inhalte und die didaktischen Formen der digitalen Medien meist nur vage formuliert (Bundesministerium für Bildung, Wissenschaft und Forschung, 2019: 2). Das Potential der Nutzung von digitalen Technologien, um das politische und soziale Umfeld der SchülerInnen zu stärken und zudem (meta)sprachliche Kompetenzen der SchülerInnen zu fördern, wird ebenso nicht ausgeschöpft. 
Schließlich ist auch die Notwendigkeit einer kritischen Auseinandersetzung mit den digitalen Medien aus den Lehrplänen nicht ersichtlich. Es werden zwar indirekt pädagogische, inhaltliche und technische Bereiche angesprochen (Mishra \& Koehler, 2006), nicht aber übergeordnete Kompetenzen für eine digitalisierte Gesellschaft ins Auge gefasst (Baumgartner et al., 2016; KMK, 2017). Damit geht ein Teil der Sinnhaftigkeit eines Medienbezugs verloren und der Fokus auf digitale Technologien läuft Gefahr, als Modeerscheinung abgetan zu werden. Daten bezüglich der Nutzung von (sozialen) Medien seitens der neuen Generation belegen das Gegenteil. Ein Blick auf den stetig wachsenden Gebrauch von SNS seitens Jugendlicher (Bitkom, 2019) spricht für die Nachhaltigkeit des Themas und ruft zum nötigen Einsatz der Bildungsbeauftragten auf. Der Zusammenhang von Gebrauch von SNS seitens Jugendlicher und ihrer Meinungsbildung ist ebenso deutlich: Neben der Lösung persönlicher Probleme (62\%) geben Jugendliche an, über SNS am meisten auf Informationen zum aktuellen Weltgeschehen (56\%) zuzugreifen. Auch Umweltthemen (40\%) sowie Bundes- und Weltpolitik (39\%) sind unter den meistgesuchten Bereichen (JIM-Studie, 2017). Wenn Jugendliche explizit gefragt werden, beurteilen sie die Glaubwürdigkeit der Informationen online konservativer als jene in Print-Medien oder TV: Nur 16\% würden im Falle widersprüchlicher Berichte der Berichterstattung im Internet eher Glauben schenken, und das trotz Steigerung der Nutzung digitaler Medien (JIM-Studie, 2005-2014). Informationen in SNS werden aber selten als ,Berichte“ wahrgenommen, da sie aus vermeintlich vertrauten und glaubwürdigen Quellen stammen und deswegen häufig Freiraum für die Zirkulation von ungenauen oder schlicht nicht fundierten Informationen lassen, die ohne kritisches Filtern angenommen werden.

Der divulgative technische Diskurs spricht von Echo Chambers (DiFonzo, 2011) oder von Filter Bubbles (Pariser, 2011) und deutet darauf hin, dass Algorithmen, personalisierte Suchergebnisse bei der Internetrecherche, sowie die ideologische Segregation in SNS die Meinungsbildung der Jugendlichen beschränken bzw. radikalisieren und die demokratische Gesellschaft polarisieren (Sunstein, 2017). Selbst wenn diese Hypothesen sich als überschätzt nachweisen lassen (Barberá, Jost, Nagler, Tucker, \& Bonneau,

Kapelari, Suzanne (Hg.), Vierte „Tagung der Fachdidaktik“ 2019:

„Interdisziplinäre fachdidaktische Diskurse zur Bildung für nachhaltige Entwicklung“

(C) 2020 innsbruck university press, ISBN 978-3-99106-019-2, DOI 10.15203/99106-019-2 
2015; Dubois \& Blank, 2018), und die genauen Einflüsse der SNS auf Jugendliche noch nicht quantitativ belegt sind (Mills, 2016), ist die Gefahr einer einfachen Manipulation (Vergeer, 2013) durch unbewusste (und daher unkritische) Informationsselektion in SNS zu groß, um von Bildungsträgern ignoriert zu werden.

Der bewusste Einsatz von pragmalinguistisch-basierten Lesekompetenzen kann die Einflüsse einer persuasiven Kommunikation minimieren und es damit Jugendlichen ermöglichen, Informationsquellen auf der Basis der eigenen Kritikfähigkeit zu lesen (Brocca \& Masia, in Vorbereitung). Somit könnten Lehrkräfte SchülerInnen dabei fördern, sich reflektiert zu informieren und mündige BürgerInnen in einer digitalisierten Welt zu werden.

\section{Die Rolle der Pragmalinguistik für das Verständnis von Überzeugungsstrategien}

Der Informationsfluss in SNS unterliegt vielfältigen Faktoren wie starkem sozialen Druck, Knappheit, Multimodalität, Anonymität der Sender und Empfänger, etc. Um das Spektrum der Variablen zu reduzieren, wird dieser Beitrag sich ausschließlich mit Nachrichten über Twitter als prototypische SNS auseinandersetzen. Twitter stellt zwar bei Jugendlichen keine so wichtige Informationsquelle wie andere SNS (z.B. WhatsApp oder Instagram) dar (vgl. JIM-Studie, 2015: 32; Jugend-Internet-Monitor, 2019), trotzdem erweist sich dieses Medium für didaktische Zwecke als besonders geeignet: In erster Linie ist Twitter motivierend, da viele Prominente (oder deren KommunikationsberaterInnen) mit ihren Accounts präsent sind. Wenn es darum geht, den SchülerInnen Einblick in das politische Spektrum zu ermöglichen, sind PolitikerInnen auf keiner anderen SNS so gut vertreten wie auf Twitter. Zudem sind im Fall von Twitter die lexikalische, syntaktische und vor allem textuelle Komplexität durch die Prägnanz und Simplifizierung der Nachrichten (die Länge der Tweets ist auf 240 Zeichen begrenzt) sehr gering gehalten. Anders als ausführlichere Nachrichtenformate politischer Kommunikation (Zeitungen,

Kapelari, Suzanne (Hg.), Vierte „Tagung der Fachdidaktik“ 2019:

„Interdisziplinäre fachdidaktische Diskurse zur Bildung für nachhaltige Entwicklung“

(C) 2020 innsbruck university press, ISBN 978-3-99106-019-2, DOI 10.15203/99106-019-2 
Blogbeiträge, usw.) ist das Leseverständnis der meisten Tweets bereits ab dem Niveau „selbständige Sprachanwendung“ (B1-B2 des Gemeinsamen Europäischen Referenzrahmen für Sprachen (GER) (Trim et al., 2001) möglich. Allerdings sind sehr hohe kulturelle Kompetenzen erforderlich, da die Bedeutung von Tweets von medialen und politischen Kontexten, sowie von der kommunikativen Situation (Stalnaker, 2002) abhängig ist.

Wie in anderen Medien auch ist die politische Kommunikation über Twitter von impliziten Diskursstrategien geprägt (Garassino, Masia, \& Brocca, 2019; Lombardi-Vallauri \& Masia, 2014a). Implizite Kommunikation ist zwar aufgrund der Sprachökonomie für die tägliche Kommunikation notwendig, weil der Sender davon ausgehen kann, dass dem Empfänger gewisse Inhalte (wie die kommunikative Situation, die Beziehung zwischen Sender und Empfänger, die Informationen, die sie teilen und ihre kommunikative Intention) bereits bekannt oder zumindest nicht diskussionsbedürftig sind. Allerdings kann implizite Kommunikation auch angewendet werden, wenn ein Inhalt diskussionsbedürftig oder weniger bekannt ist und der Sender eine gewisse Botschaft vermitteln möchte, ohne der Kritik der Empfänger ausgesetzt zu sein (Lombardi-Vallauri \& Masia, 2014b). Wenn Informationen implizit übermittelt werden, minimiert der Sender zudem seine Verantwortung für den Wahrheitswert der Information, weil er für nichts zur Verantwortung gezogen werden kann, worüber er nichts explizit gesagt hat (Sbisà, 2007). Von diesen letzten Merkmalen der impliziten Kommunikation profitiert die Sprache der Politik, wenn implizite Kommunikationsstrategien angewendet werden, um den Empfänger zu manipulieren: Der Sender strebt nicht nach einem tieferen und genaueren Verständnis der Situation, sondern er möchte andere Menschen davon überzeugen, einen anderen Standpunkt einzunehmen, der für ihn selbst vorteilhaft ist, oder sie daran zu hindern, eine gewisse Meinung zu vertreten. Die wahren Absichten des Senders bleiben dem Empfänger verborgen (Buzărnescu, 2005: 154).

Das Risiko besteht nun darin, dass gewisse Inhalte vom Empfänger unbewusst angenommen werden und unterschwellig einen Weg in sein Hintergrundwissen

Kapelari, Suzanne (Hg.), Vierte „Tagung der Fachdidaktik“ 2019:

„Interdisziplinäre fachdidaktische Diskurse zur Bildung für nachhaltige Entwicklung“

(C) 2020 innsbruck university press, ISBN 978-3-99106-019-2, DOI 10.15203/99106-019-2 
finden. Studien zur impliziten Kommunikation (Drai \& de Saussure, 2016) haben gezeigt, dass implizit übermittelte Inhalte sich leicht ins Gedächtnis einprägen, weil sie weniger bewusst und eher unterschwellig verarbeitet werden (Lombardi Vallauri \& Masia, 2014a; Sbisà, 2007). Mehrere Autoren (de Saussure, 2013; Kerbrat-Orecchioni, 1986; Pinker, Nowak, \& Lee, 2008) haben hierbei argumentiert, dass Empfänger weniger dazu neigen, den Wahrheitsgehalt von impliziten Inhalten zu bewerten, als wenn diese explizit vermittelt werden.

Implizite Kommunikation kann mittels unterschiedlicher Formen und Strategien geschehen. In diesem Beitrag wird die Untersuchung impliziter Kommunikation auf zwei kommunikative Mittel beschränkt, die besonders relevant sind und bereits ausführlich erforscht wurden (de Saussure, 2013; Ducrot, 1972; KerbratOrecchioni, 1986; Lombardi Vallauri, 2002; Reboul, 2011; Sbisà, 2007; Stalnaker, 1973). Diese Mittel sind Präsuppositionen und Implikaturen.

Nach Stalnaker (1973) ist eine Präsupposition die Information, die als gemeinsame Grundlage für Sender und Empfänger vor einem kommunikativen Akt angenommen wird. Das passiert typischerweise, wenn Sender und Empfänger gemeinsames Vorwissen teilen. Vorausgesetzte Informationen werden durch Präsuppositionen übermittelt. Präsuppositionen bedienen sich typischer lexikalischer Ausdrücke oder syntaktischer Konstruktionen als Präsuppositionsauslöser. Typische Präsuppositionsauslöser sind z.B. definite Kennzeichnungen, iterative Verben, additive Adverbien, Zustandsveränderungsverben, faktive Verben und adverbiale Nebensätze. Ein Beispiel zu jedem dieser Präsuppositionsauslöser wird hier gegeben (Levinson, 1983, deutsche Fassung 2000,S. 197-201):

(1) Definite Kennzeichnungen: Die Schokoladenschachtel ist in der Küche.

(2) Iterative Adverbien: Dein Hund hat meine Vase wieder zerbrochen.

(3) Additive Adverbien: Jane trinkt auch Milch zum Frühstück.

(4) Zustandsveränderungsverben: Mark fängt wieder an, nachts Geräusche zu machen. 
(5) Faktive Verben: Mark erkannte, dass er hoch verschuldet war.

(6) Adverbiale Nebensätze: Als meine Tochter ihren Abschluss machte, kauften wir ihr ein neues Auto.

Ein weiteres Mittel, um implizite Informationen $\mathrm{zu}$ übermitteln, ist die Implikatur. Nach Grice (1975) werden Implikaturen als beabsichtigte Bedeutungen definiert, die nicht direkt aus der Oberflächenstruktur einer Äußerung ableitbar sind, sondern nur indirekt auf der Grundlage von Kontextinformationen und Annahmen des Weltwissens abgeleitet werden können, wie im folgenden Beispiel deutlich wird:

(7) Emmanuel Macron, 28.02. 2017 über Twitter: „Dans une économie de la transformation radicale que nous sommes en train de vivre, il faut prendre des risques."

[dt.: „In einer von radikalen Veränderungen geprägten Wirtschaft, die wir gerade erleben, müssen wir Risiken eingehen." ]

Die Bedeutung dieses Tweets (also, von welchen Risiken die Rede ist und wer sie eingehen sollte) ist den heutigen LeserInnen nicht so offensichtlich wie den Followers von Macron während des Wahlkampfs 2017, da sich in der Äußerung „il faut prendre des risques“ eine Implikatur befindet. Nur mit adäquaten Kontextinformationen kann der Empfänger verstehen, dass Macrons Botschaft impliziert, dass die damalige Regierung von François Hollande ${ }^{3}$ kein Risiko eingegangen ist, um von den wirtschaftlichen Veränderungen $\mathrm{zu}$ profitieren, bzw. dass François Hollande nicht mutig genug war.

Traditionell (Levinson, 1983: 183) werden zwei Arten von Implikaturen unterschieden: konversationelle und konventionelle Implikaturen. Konventionelle Implikaturen basieren auf der logisch-semantischen Bedeutung einer Äußerung: Ein typisches Beispiel für ihren Auslöser ist die Konjunktion , aber' wie in der folgenden Äußerung:

3 Staatspräsident der Französischen Republik vor Manuel Macron.

Kapelari, Suzanne (Hg.), Vierte „Tagung der Fachdidaktik“ 2019:

„Interdisziplinäre fachdidaktische Diskurse zur Bildung für nachhaltige Entwicklung“

(C) 2020 innsbruck university press, ISBN 978-3-99106-019-2, DOI 10.15203/99106-019-2 
(8) Johannes ist reich, aber sympathisch.

Durch die adversative Bedeutung der Konjunktion ,aber' wird unterstellt, dass alle reichen Menschen unsympathisch sind. Konventionelle Implikaturen sind Inferenzen aus der Bedeutung bestimmter lexikalischer Ausdrücke (Levinson, 1983). Im Gegensatz zu konversationellen Implikaturen erfordert ihr Verständnis lediglich die Sprachkompetenz des Empfängers, ein tieferes situatives Hintergrundwissen ist nicht nötig. Bei konversationellen Implikaturen hingegen kann die kommunikative Intention des Senders nur durch die richtige Einschätzung der kommunikativen Situation verstanden werden: Dafür müssen Sender und Empfänger Vorwissen und informative Ziele teilen. Konversationelle Implikaturen können z.B. aus indirekten Aussagen oder Antworten entstehen:

(9) A: Möchtest du einen Kaffee haben?

B: Ich wollte gleich ins Bett gehen.

In diesem Fall kann A verstehen, dass B keinen Kaffee möchte, selbst wenn seine Frage nicht direkt verneint wurde, weil die Gesprächspartner das Wissen teilen, dass Kaffee das Einschlafen erschwert. Das Beispiel zeigt, wie man in einer konversationellen Implikatur „mehr meinen kann, als tatsächlich [wörtlich] gesagt wurde“ (Levinson 1983, Dt. 2000: 107).

Wie auch in anderen Bereiche der Pragmatik (Brocca, Borowiec, \& Masia, 2020: S.84; Nuzzo, 2013) wird die implizite Kommunikation kaum in sprachdidaktischen Werken behandelt und es bleibt so an der Intuition der Lernenden, pragmatische Strategien richtig zu erschließen und zu verwenden. Dies kann jedoch sprachspezifisch sehr unterschiedlich zum Ausdruck kommen. Das Erkennen und Verstehen impliziter Informationen ist in österreichischen Lehrplänen ausdrücklich festgehalten, allerdings nur in Bezug auf die muttersprachliche Bildung: 
Textkompetenz: Texte reflektieren und bewerten: durch das Erkennen von Strategien der Beeinflussung politische Mündigkeit erlangen; die Fähigkeit der Perspektivenübernahme und der kritischen Betrachtung von Perspektiven fördern. (Lehrpläne für Deutsch) (Bundesministerium für Unterricht und Kunst, 1985)

Auch im GER (Gemeinsamem europäischen Referenzrahmen für Sprachen) wird implizite Kommunikation kaum behandelt (Trim et al., 2001). Erst ab dem Niveau C1 und bei „langen und komplexen“ Texten wird die Kompetenz, implizite Inhalte zu erkennen, berücksichtigt.

Argumentation Verstehen: C1: [der Lernende] kann ein weites Spektrum langer, komplexer Texte, denen man im gesellschaftlichen, beruflichen Leben oder in der Ausbildung begegnet, verstehen und dabei feinere Nuancen auch von explizit oder implizit angesprochenen Einstellungen und Meinungen erfassen. (Trim et al., 2001)

Da kaum Erfahrungswerte vorhanden sind, wie die Didaktisierung impliziter Kommunikationsstrategien umzusetzen ist, lohnt es sich, einen Blick auf die spontane Erkennung und Behandlung von Implizitem im Alltag zu werfen. Tatsächlich werden auf Implizitem aufgebaute Argumentationen von EmpfängerInnen oft abgewehrt, indem das Implizite offengelegt wird. Im folgenden Beispiel aus der Sprache der Politik attackierte der damaliger Innenminister Matteo Salvini einen Journalisten, der den sechzehnjährigen Sohn Salvinis im Urlaub auf einem Wasserscooter der Polizei entdeckte.

(10) Matteo Salvini 01.08. 2019, Interview in Milano Marittima: "Vada a riprendere i bambini, lei che è specializzato e le piace tanto.“ [dt.: „Gehen Sie Kinder filmen, da Sie darin spezialisiert sind und es Sie so sehr freut."]

In dieser Aussage wird durch eine konversationelle Implikatur (die auf einer Übergeneralisierung aufbaut) der Journalist diskreditiert: Der Journalist hat 
einen Minderjährigen gefilmt. Durch eine explizite Inferenz wird behauptet, dass er generell Minderjährige filmen würde, was unterschwellig den Vorwurf der Pädophilie vermittelt. Für diesen implizit ausgedrückten Inhalt - die ein unkritischer Empfänger leicht als wahr akzeptieren könnte - kann der Minister nicht verantwortlich gemacht werden. Würde der Minister diesen Inhalt hingegen durch eine Assertion ausdrücken, würde die Übertreibung direkt deutlich und die ZuhörerInnen würden den Inhalt der Aussage als solche ablehnen. Der Journalist offenbart die Implikatur:

(11) Journalist: "Mi sta dando del pedofilo?”
[dt.: „Unterstellen Sie mir, pädophil zu sein?“]

Der Innenminister muss nun die Assertion ,Der Journalist ist pädophil verneinen, da die Äußerung offenbar falsch ist und er für diese Äußerung verantwortlich (und ggf. strafbar) wäre.

(12) Matteo Salvini: "No, dico solo [...]" [dt.: "Nein, ich sage nur..."]

Der Journalist hat die Implikatur seines Angreifers paraphrasiert und dabei die implizit mitvermittelte Bedeutung explizit gemacht, was eine grundlegende Veränderung der Gesprächssituation provoziert.

Ein ähnlicher Prozess kann auch in didaktischen Kontexten angewendet werden. Die Fachdidaktik nutzt bereits Paraphrasierungsaufgaben, um Lernprozesse nachzuverfolgen und Diagnosen über das Textverständnis zu treffen. Sbisà (2007) berichtet von positiven Erfahrungen bei der Erkennung von impliziten Informationen durch das Paraphrasieren von Texten, vorausgesetzt, dass in der Paraphrase die impliziten Informationen explizit wiedergegeben werden. Die o.a. Beispiele (8) und (9) könnten folgendermaßen paraphrasiert werden:

(13) Meistens sind reiche Leute unsympathisch. Aber Johannes ist reich und sympathisch. 
(14) A: Möchtest du einen Kaffee haben?

B: Nein, ich sollte und werde keinen Kaffee trinken, weil ich gleich ins Bett gehen will, und wenn ich Kaffee trinke, kann ich schlecht einschlafen.

Natürlich sind die Paraphrasierungen (13) und (14) im Alltag zwecklos und verstoßen gegen pragmalinguistische Regeln; hier werden sie lediglich zwecks der Übersichtlichkeit angeführt. Hingegen ist die Paraphrasierung von Informationen mit manipulativer Intention, wie im Dialog (10-12), der bewährteste Weg, um eine kritische Position gegenüber Informationen aufzubauen. Nur wenn eine Information asseriert wird, ist es möglich die kommunikative Intention des Senders $\mathrm{zu}$ verstehen (Sbisà, 2007) und entsprechend zu reagieren.

\section{Darstellung didaktischer Materialien}

In diesem Abschnitt werden nun didaktische Materialien präsentiert, die als Beispiel für eine kritische Medienbildung im Rahmen des Fremdsprachunterrichts Französisch eingesetzt werden können. Die Materialien decken die Themenbereiche des aktuellen Zeitgeschehens ab und ermöglichen einen Blick auf die politische Landschaft Frankreichs. Sie dienen auch dem bewussten Umgang mit impliziten Kommunikationsstrategien in digitalen und analogen Medien und erhöhen damit die rezeptive und produktive pragmalinguistische Kompetenz. Am Ende der Einheit sollten die SchülerInnen in der Lage sein, implizite Informationen zu erkennen und umzudeuten, sowie eine entsprechende Reaktion zu formulieren. Mit dem Erkennen und Verstehen von impliziten Bedeutungen erlangen die Lernenden höhere textuelle Kompetenzen in der Fremdsprache. Eine Sensibilisierung für persuasive Kommunikation in anderen Sprachen und Textsorten ist ebenfalls zu erwarten.

Die rezeptiven Kompetenzen setzen sprachlich B2-Niveau nach GER voraus, während in der Produktionsphase sprachliche Kompetenzen des Niveaus B1 die

Kapelari, Suzanne (Hg.), Vierte ,Tagung der Fachdidaktik“ 2019:

„Interdisziplinäre fachdidaktische Diskurse zur Bildung für nachhaltige Entwicklung“

(C) 2020 innsbruck university press, ISBN 978-3-99106-019-2, DOI 10.15203/99106-019-2 
Mindestanforderung sind. Damit eignen sich die Materialien thematisch und sprachlich für die Sekundarstufe II (z.B. in der 12. Schulstufe) nach mindestens vier oder fünf Jahren Fremdsprachunterricht. Die Materialien können auch in einem fachübergreifenden Unterrichtssetting oder im bilingualen Sachfachunterricht im Kontext politischer Bildung, in Deutsch oder in einem Workshop im Bereich der digitalen Medien verwendet werden. Die angewendete Methode folgt dem CLIL Ansatz (Leisen, 2015), da das Material in einem Medien- und Politik-Workshop in der Fremdsprache eingesetzt wird. Sowohl die Bereitstellung authentischer Materialien als auch die Relevanz der behandelten Inhalte regen die Produktionsarbeit auf natürliche Weise an. Neben Authentizität und Aktualität besteht der hohe Wert von CLIL-Unterricht darin, dass durch ihn eine besondere Förderung von Textkompetenz ermöglicht wird (Mentz, 2004), da eine ständige Verknüpfung von Textrezeption und -produktion stattfindet.

Der vorgeschlagene Unterricht teilt sich in drei aufeinander aufbauende Phasen: Einstieg, Erarbeitung und Sicherung. In der Einstiegsphase wird den SchülerInnen ein Text präsentiert, in dem implizit übertragene Informationen enthalten sind. Durch ein induktives Verfahren werden die SchülerInnen angeleitet die impliziten Aussagen herauszuarbeiten. Als Textgrundlage wurde eine Rede von Emmanuel Macron gewählt (s. Materialien im Anhang 1a). Der französische Präsident wandte sich in einer Fernsehansprache am 18. Dezember 2018 an die französischen BürgerInnen, um die Maßnahmen nach den Demonstrationen der Gelbwestenbewegung (Fr. mouvement des Gilets jaunes) anzukündigen. ${ }^{4}$ Nach der Aktivierung von Vorwissen werden die SchülerInnen mit einer ausführlicheren Analyse konfrontiert. Es gilt herauszufinden, ob gewisse Informationen (s. Anhang 1b) im Text erhalten sind. Dafür können die SchülerInnen die schriftliche Version des Textes bearbeiten. Abgesehen von einem Distraktor (einer Aussage, die nicht im Text vorhanden ist), sind alle

4 Das gesamte Video kann unter folgendem Link angesehen werden: https:/www.elysee.fr/emmanuel-macron/2018/12/10/adresse-du-president-de-la-republiquedu-lundi-10-decembre-2018 (12.06.2019)

Kapelari, Suzanne (Hg.), Vierte „Tagung der Fachdidaktik“ 2019:

„Interdisziplinäre fachdidaktische Diskurse zur Bildung für nachhaltige Entwicklung“

(C) 2020 innsbruck university press, ISBN 978-3-99106-019-2, DOI 10.15203/99106-019-2 
erfragten Items durch Implikaturen oder Präsuppositionen übermittelt. Nach dem Lösen der Aufgabe werden die SchülerInnen gefragt, ob die gefundenen Informationen direkt vermittelt oder im Text, versteckt ${ }^{\star}$ sind. Das Beantworten dieser Fragen führt in eine kurze, induktive Informationsphase im Plenum, in der die SchülerInnen Metakompetenzen zu impliziten Kommunikationsstrategien anwenden und ein Bewusstsein für implizite Kommunikation entwickeln. Die SchülerInnen sollten danach in der Lage sein, zu erkennen, dass das Verständnis von impliziten Kommunikationsstrategien die Grundlage für gelungene Kommunikation ist (Sbisà, 2007, S. 6). In dieser Phase können z.B. typische Präsuppositionsauslöser erkannt und aufgelistet werden.

In der Erarbeitungsphase werden die SchülerInnen aufgefordert, selbst implizite Botschaften zu assertiven Äußerungen umzuwandeln und dazu eine kritische Stellungnahme zu formulieren. Der Bezug zur Lebenswelt kann hier durch Verweis auf die Interviewvorbereitung kritischer JournalistInnen oder politischer BeraterInnen oder der Einrichtung eines Fact-Checking Blogs (Brocca, Masia \& Will, in Vorbereitung) hergestellt werden. SchülerInnen werden ausgewählte Tweets vorgelegt, die Implikaturen oder Präsuppositionen beinhalten. Durch die Paraphrasierung der Tweets sollte die implizite Botschaft anfechtbar werden, wie in folgendem Beispiel verdeutlicht wird:
(15)
Jean-Luc Mèlenchon 03.05. 2017 über Twitter: „Il faut une éthique républicaine sans faille dans la police!“
[dt.: „Man braucht einwandfreie Verfassungsloyalität in der Polizei“]

Die Äußerung beinhaltet eine Implikatur, die durch ein verbe impersonnel ausgelöst wird. „Il faut“ (dt. es ist notwendig, man braucht) impliziert, dass das Objekt „une éthique républicaine sans faille“ nicht vorhanden ist. Eine mögliche Paraphrasierung durch eine Assertion könnte folgendermaßen lauten: 


\section{(16) Die Verfassungstreue der Polizei hat Lücken.}

Je nach Reife des didaktischen Kontexts sind mehrere kritische Auseinandersetzungen möglich wie z.B. ,Waren einige Polizisten nicht loyal oder sind sie generell nicht verfassungsloyal gewesen?' Weitere Beispiele werden tabellarisch in Anhang 2 dargestellt.

In der Sicherungsphase sollen die SchülerInnen das Erlernte in einem freieren Kontext anwenden können. Mithilfe von SNS mit pädagogischem Hintergrund und höheren Sicherheitsstandards, wie z.B. Fakebook oder Twiducate ${ }^{5}$ (Alvermann, 2017), könnten die SchülerInnen in einer geschlossenen Gruppe die Erfahrung von politischen Diskussionen in SNS erleben. Den SchülerInnen können hier auch durch Eckdaten von fiktiven BürgerInnen Rollen zugeteilt werden. Sie können dann ihr fiktives Profil in der Zielsprache ausführlicher bearbeiten und online mit PartnerInnen teilen. Anschließend könnten sie in einem Rollenspiel gewisse Positionen vertreten. Dadurch können die Lernenden auf die sprachliche Produktion fokussieren, ohne ihre eigene Idee vertreten zu müssen, und sie werden nicht gezwungen, ihre politische Orientierung zu offenbaren (bzw. eine eigene zu übernehmen). Die SchülerInnen werden in dieser Phase dazu angeregt, keine impliziten Äußerungen in ihre Argumente einzubauen bzw. die impliziten Botschaften anderer MitschülerInnen zu dementieren oder zu hinterfragen. Das lexikalische und textuelle Material der vorherigen Phasen kann und soll in der Textproduktion (Posts oder Tweets) wiederverwendet werden.

Der in dieser Phase mögliche thematische Anknüpfungspunkt an das kommunikative Verhalten in Debatten und Konfliktgesprächen on- und offline (Bundeszentrale für die politische Bildung, 2018; Fluter, 2017) bietet Raum für Aufklärung über die potentielle Aggressivität von Äußerungen mit impliziten Diskursmitteln (Janetzko, 2017; Piskorska, 2017). Es sollte dabei klar gestellt

5 Fakebook und Twiducate sind jeweils unter folgenden Adressen aufzurufen https://www.classtools.net/FB/home-page und https://www.livelingua.com/twiducate/.

Kapelari, Suzanne (Hg.), Vierte „Tagung der Fachdidaktik“ 2019:

„Interdisziplinäre fachdidaktische Diskurse zur Bildung für nachhaltige Entwicklung“

(C) 2020 innsbruck university press, ISBN 978-3-99106-019-2, DOI 10.15203/99106-019-2 
werden, dass, selbst wenn Meinungen mit Fakten belegt sind oder direkt asseriert werden, Meinungsdivergenzen unvermeidlich sind. Die Abschaffung impliziter Kommunikation soll nicht zum Ziel werden, weil (i) implizite Kommunikation unvermeidbar bzw. notwendig ist und (ii) explizite Kommunikation nicht immer das geeignete Instrument ist, konfliktfreie Kommunikation zu etablieren. Die Lernenden sollten ,lediglich“ befähigt werden, implizite Kommunikationsmittel zu erkennen, vor allem, wenn diese manipulatorische Zwecke verfolgen, und eine Abwehrstrategie (z.B. das Paraphrasieren und Hinterfragen von Aussagen) entwickeln, um andere Menschen und deren persönliche Meinung besser zu verstehen (Sbisà, 2007, S. 201). Somit soll es den SchülerInnen letztlich leichter fallen, ihre eigene Meinung zu bilden und diese respektvoll zu äußern.

\section{Fazit}

Die Rolle von SNS im fachdidaktischen Diskurs zur Bildung für nachhaltige Entwicklung ist erheblich. SNS stellen eine Chance für die heutigen Generationen dar, weil sie den Kommunikationsfluss beschleunigen und neue Formen der Kommunikation einführen. Sie finden auch in der politischen Kommunikation zunehmend Anwendung und haben einen starken Einfluss auf die junge Generation (Stodt, Wegmann, \& Brand, 2015). Trotzdem können die vom Medium bediente Geschwindigkeit und Knappheit die Komplexitätsreduzierung legitimieren; diese kann wiederum mit Manipulationsabsichten angewendet werden. Die Bewusstmachung von Manipulationstechniken sowie der Erwerb hinterfragender Lesestrategien verbessern die Rezeption von SNS und stärken gleichzeitig die demokratische Teilhabe im Sinne der SDGs. Die Frage, ob und wie ihre Rolle in der Unterrichtspraxis thematisiert werden soll, ist zugleich eine didaktische und eine gesellschaftliche Frage.

Ziel der allgemeinbildenden höheren Schulen Österreichs ist laut den Lehrplänen, junge Menschen zu befähigen, mündige Bürger eines demokratischen und von Digitalisierung beeinflussten Systems zu werden:

Kapelari, Suzanne (Hg.), Vierte „Tagung der Fachdidaktik“ 2019:

„Interdisziplinäre fachdidaktische Diskurse zur Bildung für nachhaltige Entwicklung“

(C) 2020 innsbruck university press, ISBN 978-3-99106-019-2, DOI 10.15203/99106-019-2 
Digitale Kompetenz, Medienkompetenz sowie politische Kompetenzen [...] haben das Ziel eines informierten, souveränen und verantwortlichen Umgangs mit Medien und Technik durch mündige Bürgerinnen und Bürger in der Demokratie und einer zunehmend von Digitalisierung beeinflussten Gesellschaft. Im Mittelpunkt steht dabei die reflektierte Verwendung von Medien [...]. (Bundesministerium für Unterricht und Kunst, 1985: Fassung vom 01.09.2018)

$\mathrm{Zu}$ den Kompetenzen, die Jugendliche auf eine digitale Gesellschaft vorbereiten, gehört die Fähigkeit, Nachrichten aus SNS kritisch zu lesen und vertrauenswürdige bzw. wahre Informationen aus dem Netz zu filtern. Der Fremdsprachenunterricht mit CLIL-Ansatz kann einen günstigen Bezugsrahmen für die Förderung der reflektierten Medienbildung darstellen, u.a. weil der Fremdsprachenunterricht eine emotionale Distanz $\mathrm{zu}$ politischen Themen ermöglicht, die im muttersprachlichen Unterricht ggf. schwieriger aufrecht zu erhalten wäre. In der kritischen Auseinandersetzung mit Medien soll die Analyse der impliziten Kommunikation eine wichtige Rolle übernehmen, weil diese es ermöglicht, die SchülerInnen zu befähigen, in SNS implizit vermittelte Informationen $\mathrm{zu}$ entschlüsseln und damit Inhalte tiefgreifender $\mathrm{zu}$ verstehen, logisch nachzuvollziehen oder kritisch zurückzuweisen. Die Analyse der impliziten Kommunikation ist wissenschaftlich auf einer soliden pragmalinguistischen Basis gegründet und gleichzeitig praxisorientiert und kann in unterschiedlichen Stufen und Fächern geübt und regelmäßig eingesetzt werden.

Die Beispiele in Abschnitt 4 haben im Französischunterricht für die Sekundarstufe II auf das Verstehen von politischen Botschaften fokussiert, indem ein altersangemessenes Thema (politische Kommunikation) mit den nötigen fremdsprachlichen Kompetenzen in Übereinstimmung gebracht ist. Neben sprachlichen und (inter-)kulturellen Zielen versucht dieser Beitrag auch ein fächerübergreifendes Ziel in Anlehnung an die Lehrpläne (s. Zitat oben) zu erreichen: Die aktive politische Teilhabe der SchülerInnen nachhaltig zu fördern und es ihnen zu ermöglichen, verantwortungsbewusste BürgerInnen in der 
digitalen Demokratie zu werden. Des Weiteren versucht dieser Aufsatz einen Beitrag dazu zu leisten, diese Generation zukunftsfähig zu machen und sie zu befähigen, Manipulation online zu erkennen und abzuwehren.

Die Kompetenzen im Umgang mit impliziten Kommunikationsstrategien können auch im früheren Alter Anwendung finden, wenn die fremdsprachlichen Herausforderungen reduziert werden und die Themen altersgerecht gewählt sind. Die Themen können weitere Bereiche über das Verständnis von politischer Kommunikation hinaus abdecken, von der impliziten Kommunikation in der Werbung bis hin zu Konflikten in privaten Gesprächen. Positive Wirkungen können so im Vermeiden von verbaler Aggression oder Cyber-Mobbing erwartet werden, da das Erkennen von impliziter Kommunikation helfen kann, andere $\mathrm{zu}$ verstehen und $\mathrm{zu}$ respektieren, sowie seine eigene Meinung zum Ausdruck zu bringen und diese selbstbewusst zu vertreten. Das ist nicht nur ein Ziel für eine nachhaltige Bildung, sondern auch für eine nachhaltige gesellschaftliche Entwicklung. ${ }^{6}$

\section{Anhang}

Anhang 1: Beispiel einer Didaktisierung einer politischen Rede. Mögliche Antworten der SchülerInnen sind kursiv geschrieben. Die Auslöser von impliziten Inhalten sind zwecks Übersichtlichkeit für Lehrkräfte fett markiert.

6 Das dargestellte Beispiel wird künftig an konkrete Klassensituationen adaptiert und getestet. Interessiertes Lehrpersonal wird herzlich eingeladen die Materialien mit zu entwickeln und zu erproben. Der Autor freut sich auf Kommunikation unter

Mail: nicola.brocca@uibk.ac.at

Twitter: @nicola_brocca

https://uibk.academia.edu/nicolabrocca

Kapelari, Suzanne (Hg.), Vierte „Tagung der Fachdidaktik“ 2019:

„Interdisziplinäre fachdidaktische Diskurse zur Bildung für nachhaltige Entwicklung“

(C) 2020 innsbruck university press, ISBN 978-3-99106-019-2, DOI 10.15203/99106-019-2 
a) Le discours d'Emmanuel Macron face aux gilets jaunes. 10 décembre 2018

"Françaises, Français,

[...] Nous avons tous vu le jeu des opportunistes (definite Kennzeichnung $\rightarrow>$ Präsupposition) qui ont essayé de profiter des colères sincères pour les dévoyer. Nous avons tous vu les irresponsables politiques (definite Kennzeichnung $\rightarrow>$ Präsupposition) dont le seul projet était de bousculer la République, cherchant le désordre et l'anarchie. Aucune colère ne justifie qu'on s'attaque à un policier (konversationelle Implikatur), à un gendarme, qu'on dégrade un commerce ou des bâtiments publics. Notre liberté n'existe que parce que chacun peut exprimer ses opinions, que d'autres peuvent ne pas les partager sans que personne n'ait à avoir peur de ces désaccords (konversationelle Implikatur). Quand la violence se déchaîne, la liberté cesse (Zustandsveränderungsverb $\rightarrow$ Präsupposition). C'est donc désormais le calme et l'ordre républicain qui doivent régner (Faktiver Verb und Zeitabverb -> Präsupposition) pourçarien ne se construira de durable tant qu'on aura des craintes pour la paix civile.

Ce sont quarante années de malaise qui ressurgissent: malaise des travailleurs qui ne s'y retrouvent plus (Zustandsveränderungsadverb à Präsupposition); malaise des territoires, villages comme quartiers où on voit les services publics se réduire (Zustandsveränderungsverb $\rightarrow$ Präsupposition) [...]. Nous voulons bâtir (Zustandsveränderungsverb $\rightarrow$ Präsupposition) une France du mérite, du travail, une France où nos enfants vivront mieux que nous. Cela ne peut se faire que par une meilleure école, des universités, de l'apprentissage et des formations qui apprennent aux plus jeunes et aux moins jeunes ce qu'il faut pour vivre libre et travailler. Nous voulons une France où l'on peut vivre dignement de son travail (konversationelle Implikatur).

Je reviendrai m'exprimer devant vous pour vous rendre compte. Mon seul souci, c'est vous; mon seul combat, c'est pour vous. Notre seule bataille, c'est pour la France.

Vive la République, vive la France!"

Textauszug aus:

https://www.elysee.fr/emmanuel-macron/2018/12/10/adresse-du-president-de-la-republique-du-lundi-10decembre-2018

Kapelari, Suzanne (Hg.), Vierte „Tagung der Fachdidaktik“ 2019:

„Interdisziplinäre fachdidaktische Diskurse zur Bildung für nachhaltige Entwicklung“

(C) 2020 innsbruck university press, ISBN 978-3-99106-019-2, DOI 10.15203/99106-019-2 
b) Après avoir lu le discours du President Macron, décide quell message n'est pas transmis par le texte:

1. Il y a des opportunistes.

....Nous avons tous vu le jeu des opportunistes .....

2. Des manifestants en colère ont attaqué un policier.

.... Aucune colère ne justifie qu'on s'attaque à un policier .....

3. Pour le moment, il n'y a pas de calme.

... C'est donc désormais le calme que doit régner ...

4. Pour le moment, il y a des craintes pour la paix civile.

...rien ne se construira de durable tant qu'on aura des craintes pour la paix civile...

5. Les milieux sociaux se sont unis.

Nicht vorhanden / pas trasmis

6. Actuellement, la France n'est pas un pays méritocratique.

...Nous voulons bâtir une France du mérite...

c) Comment ces informations sont-elles transmises? 
Anhang 2: Beispiele von Tweets, die implizite Informationen beinhalten, mögliche Paraphrasierung und mögliche kritische Nachfragen.

\begin{tabular}{|c|c|c|c|}
\hline Originaler Tweet & $\begin{array}{l}\text { Finden der } \\
\text { Implizite }\end{array}$ & Paraphrasierung & $\begin{array}{l}\text { Kritische } \\
\text { Nachfragen }\end{array}$ \\
\hline $\begin{array}{l}\text { “L'agriculture bio } \\
\text { est plus chère. Si } \\
\text { on augmente les } \\
\text { salaires, les gens } \\
\text { pourront acheter } \\
\text { de meilleurs } \\
\text { produits. “Jean- } \\
\text { Luc Mèlenchon } \\
03.15 .2017\end{array}$ & $\begin{array}{l}\text { „L'agriculture bio } \\
\text { est plus chère. Si } \\
\text { on augmente les } \\
\text { salaires, les gens } \\
\text { pourront acheter } \\
\text { de meilleurs } \\
\text { produits.“ } \\
\text { Durch eine } \\
\text { konversationelle } \\
\text { Implikatur wird } \\
\text { implizit die } \\
\text { Information } \\
\text { übertragen, dass } \\
\text { Bioprodukte für } \\
\text { die Konsumenten } \\
\text { besser sind als } \\
\text { konventionelle } \\
\text { Erzeugnisse. Die } \\
\text { bessere Qualität } \\
\text { der Bioprodukte } \\
\text { sowie die } \\
\text { Korrelation von } \\
\text { Einkommen und } \\
\text { Konsum von Bio- } \\
\text { Produkten wird als } \\
\text { selbstverständlich } \\
\text { unterstellt. }\end{array}$ & $\begin{array}{l}\text { „Wenn wir die } \\
\text { Löhne erhöhen, } \\
\text { können sich die } \\
\text { Menschen Bio- } \\
\text { Produkte leisten. } \\
\text { Bio-Produkte sind } \\
\text { besser. " }\end{array}$ & $\begin{array}{l}\text { Sind Bio-Produkte } \\
\text { wirklich besser? } \\
\text { Wie genau? Sind } \\
\text { sie nachhaltiger? } \\
\text { Gesünder? } \\
\text { Warum? } \\
\text { Konsumieren die } \\
\text { Menschen mehr } \\
\text { Bio-Produkte, } \\
\text { wenn sie mehr } \\
\text { verdienen? }\end{array}$ \\
\hline
\end{tabular}




\begin{tabular}{|c|c|c|c|}
\hline $\begin{array}{l}\text { "Le patriotisme } \\
\text { économique } \\
\text { protègera nos } \\
\text { entreprises } \\
\text { stratégiques, et } \\
\text { celles qui } \\
\text { subissent une } \\
\text { concurrence } \\
\text { déloyale!" Marin } \\
\text { e Le Pen } \\
03.13 .2017\end{array}$ & $\begin{array}{l}\text { „Le patriotisme } \\
\text { économique } \\
\text { protègera nos } \\
\text { entreprises } \\
\text { stratégiques, et } \\
\text { celles qui } \\
\text { subissent une } \\
\text { concurrence } \\
\text { déloyale! '.. } \\
\text { Durch einen } \\
\text { restriktiven } \\
\text { Relativsatzt wird } \\
\text { eine } \\
\text { Präsupposition } \\
\text { ausgelöst. Es wird } \\
\text { implizit die } \\
\text { Information } \\
\text { vermittelt, dass } \\
\text { ausländische } \\
\text { Unternehmen } \\
\text { unlauteren } \\
\text { Wettbewerb } \\
\text { betreiben. }\end{array}$ & $\begin{array}{l}\text { „Es gibt } \\
\text { ausländische } \\
\text { Unternehmen, die } \\
\text { unlauteren } \\
\text { Wettbewerb } \\
\text { betreiben.“ }\end{array}$ & $\begin{array}{l}\text { Was ist } \\
\text { unlauterer } \\
\text { Wettbewerb? } \\
\text { Wer macht es? } \\
\text { Betreibt } \\
\text { Frankreich auch } \\
\text { unlauteren } \\
\text { Wettbewerb im } \\
\text { Ausland? Würde } \\
\text { ein europaweiter } \\
\text { Mindestlohn den } \\
\text { Wettbewerb } \\
\text { reduzieren? }\end{array}$ \\
\hline $\begin{array}{l}\text { „Le vrai } \\
\text { patriotisme } \\
\text { économique, c'est } \\
\text { d'abord d'être } \\
\text { compétitif“' } \\
\text { Macron } \\
03.13 .2017 \\
\text { Als Antwort auf } \\
\text { den vorherigen } \\
\text { Le Pens Tweet. }\end{array}$ & $\begin{array}{l}\text { „Le vrai } \\
\text { patriotisme } \\
\text { économique, c'est } \\
\text { d'abord d'être } \\
\text { compétitif. “ } \\
\text { Der Modifikator } \\
\text { „le vrai“ impliziert } \\
\text { (konventionelle } \\
\text { Implikatur), dass } \\
\text { es auch falschen } \\
\text { Patriotismus gibt. }\end{array}$ & $\begin{array}{l}\text { „Es gibt falschen } \\
\text { ökonomischen } \\
\text { Patriotismus (den } \\
\text { von Le Pen). “ }\end{array}$ & $\begin{array}{l}\text { Warum ist } \\
\text { Kompetitiv-sein } \\
\text { patriotisch? } \\
\text { Warum ist der } \\
\text { andere } \\
\text { ökonomische } \\
\text { Patriotismus } \\
\text { falsch? Was } \\
\text { bedeutet } \\
\text { ökonomischer } \\
\text { Patriotismus? }\end{array}$ \\
\hline
\end{tabular}

Kapelari, Suzanne (Hg.), Vierte „Tagung der Fachdidaktik“ 2019:

„Interdisziplinäre fachdidaktische Diskurse zur Bildung für nachhaltige Entwicklung“

(C) 2020 innsbruck university press, ISBN 978-3-99106-019-2, DOI 10.15203/99106-019-2 


\section{Literatur}

BBWF, Bundesministerium für Bildung, Wissenschaft und Forschung (2019). Masterplan Digitalisierung. Abgerufen am 12.12.2019

https://bildung.bmbwf.gv.at/schulen/schule40/masterplan_digitalisierung_pi.pd f? $6 \mathrm{ma} 2 \mathrm{fg}$

Bundesministerium für Unterricht und Kunst, 1985. Gesamte Rechtsvorschrift für Lehrpläne - allgemeinbildende höhere Schulen, Fassung vom 01.09.2018. Verordnung des Bundesministers für Unterricht und Kunst vom 14. November 1984 über die Lehrpläne der allgemeinbildenden höheren Schulen;

https://www.ris.bka.gv.at/GeltendeFassung.wxe?Abfrage=Bundesnormen\&Ge setzesnummer $=10008568 \&$ FassungVom $=2018-09-01$

Alvermann, D. E. (2017). Social Media Texts and Critical Inquiry in a Post Factual Era. Journal of Adolescent \& Adult Literacy, 61, 338. doi:10.1002/jaal.694

Barberá, P., Jost, J. T., Nagler, J., Tucker, J. A., \& Bonneau, R. (2015). Tweeting From Left to Right: Is Online Political Communication More Than an Echo Chamber? Spychological Science, 26(10), 1531-1542. doi: https://doi.org/10.1177/0956797615594620

Baumgartner, P., Brandhofer, G., Ebner, M., Gradinger, P., \& Korte, M. (2016). Medienkompetenz fördern - Lehren und Lernen im digitalen Zeitalter. In M. Bruneforth, F. Eder, K. Krainer, C. Schreiner, A. Seel, \& C. Spiel (Eds.), Nationaler Bildungsbericht Österreich 2015 (Vol. Band 2 - Alle Analysen im Detail pp. 95-132). Graz: Leykam.

Bundesministerium für Bildung, Wissenschaft und Forschung (2019). Masterplan Digitalisierung. Abgerufen am 12.12.2019

https://bildung.bmbwf.gv.at/schulen/schule40/masterplan digitalisierung pi.pd f? $6 \mathrm{ma} 2 \mathrm{fg}$

Bundeszentrale für die politische Bildung (2018). Hate Speech. Abgerufen am 12.12.2019

http://www.bpb.de/lernen/digitale-bildung/medienpaedagogik/231236/hatespeech

Bitkom (2019). Mit 10 Jahren haben die meisten Kinder ein eigenes Smartphone. Abgerufen am 12.12.2019 von

https://www.bitkom.org/Presse/Presseinformation/Mit-10-Jahren-haben-diemeisten-Kinder-ein-eigenes-Smartphone

Brocca, N., \& Masia, V. (in Vorbereitung). Supporting reading-understanding of implicit communication. Experimental Evidences

Kapelari, Suzanne (Hg.), Vierte „Tagung der Fachdidaktik“ 2019:

„Interdisziplinäre fachdidaktische Diskurse zur Bildung für nachhaltige Entwicklung“

(C) 2020 innsbruck university press, ISBN 978-3-99106-019-2, DOI 10.15203/99106-019-2 
Brocca, Nicola; Borowiec, Ewa und Masia, Viviana (2020). Didactics of pragmatics as a way to improve social media liter-acy. An experiment proposal with Polish and Italian students in L2. In: heiEDUCATION Journal 5 |2010, S. 81-107

Brocca, N., Masia, V., \& Will, L. (in Vorbereitung). Make pragmatics great again! Teaching implicatures and presuppositions for democracy education in the age of Twitter and Facebook. Educating the Global Citizen: International Perspectives on Foreign Language Teaching in the Digital Age 28. März 2019, Ludwig-Maximilians-Universität München.

Buzărnescu, Ş. (2005). Public sociology - conceptual system and research methodology.

DAK-Gesundheit, \& UKE, Deutsches Zentrum für Suchtfragen am Universitätsklinikum Hamburg-Eppendorf (2018). WhatsApp, Instagram und Co. - so süchtig macht Social Media. DAK-Studie: Befragung von Kindern und Jugendlichen zwischen 12 und 17 Jahren. Befragung der forsa Gesellschaft für Sozialforschung und statistische Analysen GmbH, https://www.saferinternet.at/fileadmin/redakteure/Footer/Studien/dak-studiesocial-media-nutzung-1968596.pdf.

de Saussure, L. (2013). Background relevance. Journal of Pragmatics, 59, 189. doi:10.1016/j.pragma.2013.08.009

DiFonzo, N. (2011). The Echo-Chamber Effect. The New York Times. Abgerufen am 12.12.2019 von https://www.nytimes.com/roomfordebate/2011/04/21/barack-obama-and-thepsychology-of-the-birther-myth/the-echo-chamber-effect

Drai, N., \& de Saussure, L. (2016). Quand l'implicite devient explicite: d'un accident expérimental à une étude pilote. Syntaxe \& Sémantique, 17, 115-133.

Dubois, E., \& Blank, G. (2018). The echo chamber is overstated: the moderating effect of political interest and diverse media. Information, Communication \& Society, 1-17. doi:10.1080/1369118X.2018.1428656

Ducrot, O. (1972). Dire et ne pas dire : principes de sémantique linguistique. Paris: Hermann.

Fluter-Nr.63. (2017). Propaganda. Bonn: Juni 2017.

Garassino, D., Masia, V., \& Brocca, N. (2019). Tweet as you speech. The role of implicit strategies and pragmatic functions in political communication: Data from a diamesic comparison. In: RILA, Rassegna Italiana di Linguistica Applicata, Nr. 2-3-2019 - anno LI.

Grice, P. H. (1975). Logic and conversation. In P. Cole \& J. Morgan (Eds.), Syntax and Semantics 3: Speech Acts (pp. 41-58). New York: Academic Press.

Janetzko, D. (2017). Manipulation in sozialen Netzwerken. Deutschland und Europa, Zeitschrift für Gemeinschaftskunde, Geschichte und Wirtschaft 74-2017. Landeszentrale für Politische Bildung. Baden-Württemberg. (Die neuen

Kapelari, Suzanne (Hg.), Vierte „Tagung der Fachdidaktik“ 2019:

„Interdisziplinäre fachdidaktische Diskurse zur Bildung für nachhaltige Entwicklung“

(C) 2020 innsbruck university press, ISBN 978-3-99106-019-2, DOI 10.15203/99106-019-2 
Medien und die politische Meinungsbildung. »Fake News« - ein Produkt der neuen Medien?), 26-36. Abgerufen am 12.12.2019 von www.deutschlandundeuropa.de

JIM-Studie (2015). Jugend-Information-(Multi-)Media. Basisstudie zum Medienumgang 12- bis 19-Jähriger in Deutschland. Abgerufen am 12.12.2019 https://www.mpfs.de/studien/jim-studie/2015/

JIM-Studie (2016). Jugend-Information-(Multi-)Media. Basisstudie zum Medienumgang 12- bis 19-Jähriger in Deutschland. Abgerufen am 12.12.2019 https://www.mpfs.de/studien/jim-studie/2016/

JIM-Studie (2017). Jugend, Information, Medien. Basisuntersuchung zum Medienumgang 12- bis 19-Jährigen. Abgerufen am 12.12.2019 https://www.mpfs.de/studien/jim-studie/2017/

Jugend-Internet-Monitor (2019). Welche Sozialen Netzwerke nutzen Österreichs Jugendliche? . Saferinternet.at. Abgerufen am 12.12.2019 https://www.saferinternet.at/services/jugend-internet-monitor/

Kerbrat-Orecchioni, C. (1986). L' implicite: Paris : Colin.

KMK (2017). Bildung in der digitalen Welt. Strategie der Kultusministerkonferenz.

Leisen, J. (2015). Lehrmaterialien im CLIL-Unterricht. Zeitschrift für Interkulturellen Fremdsprachenunterricht, $20(<\mathrm{b}>\mathrm{E}-\mathrm{ISSN}:</ \mathrm{b}>1205-6545)$.

Levinson, S. C. (1983). Pragmatics (1. publ.. ed.): Cambridge [u.a.] : Cambridge Univ. Press.

Lombardi Vallauri, E. (2002). La struttura informativa dell'enunciato (1. ed.. ed.): [Firenze] : La Nuova Italia Ed.

Lombardi Vallauri, E., \& Masia, V. (2014a). Implicitness impact: measuring texts. Journal of Pragmatics, 61, 161-184.

Lombardi Vallauri, E., \& Masia, V. (2014b). Misurare l'informazione implicita nella propaganda politica italiana. In R. Librandi \& R. Piro (Eds.), L'italiano della politica e la politica per l'italiano. Atti del XI Convegno ASLI Associazione per la Storia della Lingua Italiana (Napoli, 20-22 novembre 2014). Firenze: Franco Cesati Editore.

Mentz, O. (2004). Bilingualer Unterricht mit der Zielsprache Französisch. Eine Untersuchung über die aktuelle Situation in Deutschland. In F.-J. Meißner, J. Mertens, \& M. Reinfried (Eds.), Französisch heute 35/2 (pp. 122-133).

Mills, K. L. (2016). Possible Effects of Internet Use on Cognitive Development in Adolescence. Media and Communication, 4(3), 4-12.

Mishra, P., \& Koehler, M. J. (2006). Technological Pedagogical Content Knowledge: A new framework for teacher knowledge. Teachers College Record, 108 (6), 1017-1054. 
Nuzzo, E. (2013). La pragmatica nei manuali d'italiano L2: una prima indagine sull'atto linguistico del ringraziare. Revista De Italianística, 2 (26) 5-29. Abgerufen am 12.12.2019 von https://doi.org/10.11606/issn.2238-8281.v2i26p5-29

Pariser, E. (2011). The filter bubble: what the Internet is hiding from you (1. publ.. ed.): London : Viking.

Pinker, S., Nowak, M. A., \& Lee, J. J. (2008). The logic of indirect speech. Proceedings of the National Academy of Sciences of the United States of America, 105(<b $>$ E-ISSN $:</ \mathrm{b}>1091-6490)$. doi:10.1073/pnas.0707192105

Piskorska, A. (2017). On the strength of explicit and implicit verbal offences: A relevance-theoretic view. In S. Bonacchi (Ed.), Verbale Aggression: Multidisziplinäre Zugänge zur verletzenden Macht der Sprache (pp. 51-72). Berlin, Boston: De Gruyter.

Reboul, A. (2011). A relevance-theoretic account of the evolution of implicit communication. Studies in Pragmatics, 13 1-19.

Sbisà, M. (2007). Detto non detto: le forme della comunicazione implicita (1. ed. ed.). Roma [etc.]: GLF editori Laterza.

Stalnaker, R. (1973). Presuppositions. Published under the Auspices of the Association for Symbolic Logic, $2(<\mathrm{b}>$ ISSN: $\quad</ \mathrm{b}>0022-3611), \quad 457$. doi:10.1007/BF00262951

Stalnaker, R. (2002). Common ground. Linguistics and Philosophy, 25, 701-721

Stodt, B., Wegmann, E., \& Brand, M. (2015). Geschickt geklickt?! Zum Zusammenhang von Internetnutzungskompetenzen, Internetsucht und Cybermobbing bei Jugendlichen und jungen Erwachsenen. Leipzig: VISTAS Verlag.

Sunstein, C. R. (2017). \#Republic: divided democracy in the age of social media. Princeton, Oxford: Princeton University Press.

Trim, J., North, B., \& Coste D. (2001). Gemeinsamer europäischer Referenzrahmen für Sprachen: lernen, lehren, beurteilen. Langenscheidt, 2001, ISBN 978-3-468-49469-7, goethe.de [abgerufen am 20. Dezember 2019]

Vereinigte Nationen (2015) Transformation unserer Welt: die Agenda 2030 für nachhaltige Entwicklung. Resolution 69/315, UN-Generalversammlung, 10. September 2015

Vergeer, M. (2013). From echo chamber to persuasive device? Rethinking the role of the Internet in campaigns. New media \& society., 15 (, 1461-7315), 127.

Kapelari, Suzanne (Hg.), Vierte „Tagung der Fachdidaktik“ 2019:

„Interdisziplinäre fachdidaktische Diskurse zur Bildung für nachhaltige Entwicklung“

(C) 2020 innsbruck university press, ISBN 978-3-99106-019-2, DOI 10.15203/99106-019-2 


\section{Kulturelle Vielfalt in Österreichs Klassenzimmern: Welchen Beitrag kann eine Didaktik der Mehrkulturalität zur langfristig lernwirksamen Förderung von Lernenden im Englischunterricht der österreichischen Sekundarstufe I leisten?}

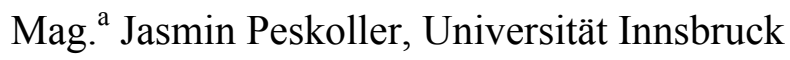

\section{Einleitung}

In diesem Beitrag wird ein Forschungsprojekt vorgestellt, das sich zum Ziel setzt, ein innovatives Unterrichtsdesign zu entwickeln, das auf die zunehmende kulturelle Vielfalt der Lernenden im Schulalltag eingeht und die Chancen nützt, die in dieser steigenden Mehrkulturalität stecken. Zunächst werden der Hintergrund der Studie sowie die schulischen Gegebenheiten mit Fokus auf den österreichischen Bildungskontext dargelegt. Im Anschluss wird der Stand der Forschung sowie Desiderata mit Schwerpunkt auf den Bereich der Sprachdidaktik angerissen und folglich ein Bezug $\mathrm{zu}$ den Sustainable Development Goals (SDGs) (siehe Rieckmann in diesem Band) hergestellt. Schließlich wird das Forschungsprojekt in seiner Konzeption präsentiert sowie dessen Zielsetzung der Anbahnung von mehrkulturellen Lernprozessen im Englischunterricht der Sekundarstufe I dargelegt. 


\section{Hintergrund}

In Zeiten von Globalisierung, Migration und Internationalisierung tritt täglich eine Vielzahl an Sprachen und Kulturen miteinander in Kontakt. Seit 2010 leben im deutschsprachigen Europa Menschen aus beinahe allen Regionen der Welt (Gogolin, 2016, S. 61). Mit dieser Entwicklung geht eine Zunahme der sprachlichen, kulturellen und sozialen Heterogenität der Bevölkerung einher (Sturm, 2016), die dazu führt, dass die Schule zu einem Ort der Mehrsprachigkeit und Mehrkulturalität wird (Bierwirth, Blell, \& Fuchs, 2017). In Bezug auf die weltweit zunehmende Vielfalt und Heterogenität im Bildungsbereich bekräftigt der australische Sprachdidaktiker Joseph Lo Bianco: "[T]he strongest indicator of the transformed realities of contemporary education in a globalised world is the depth of cultural, racial and linguistic diversity in schools" (Lo Bianco, 2009, S. 113).

Diese steigende Diversität in Gesellschaft und Schule spiegelt sich auch in Österreichs Klassenzimmern wider. Statistische Kennzahlen zeigen, dass in den Jahren 2011 bis 2016 der Prozentsatz an Menschen, die in Österreich leben, aber außerhalb Österreichs geboren wurden, von 15,7\% auf 18,8\% gestiegen ist (Statistik Austria, 2018a). Außerdem gaben in der letzten Volkszählung insgesamt 2,02 Millionen Menschen in Österreich an, einen Migrationshintergrund zu haben, was 23,3\% der Gesamtbevölkerung Österreichs entspricht (Statistik Austria, 2019). Die folgenden Abbildungen visualisieren das Ausmaß und die Entwicklung von Mehrsprachigkeit und Mehrkulturalität im österreichischen Bildungssystem anhand von statistischen Daten aus den Schuljahren 2017/18 sowie 2007/08 (Statistik Austria, 2018b). An dieser Stelle ist anzumerken, dass sich aus dem in den Abbildungen verwendeten Begriff ,Erstsprache nicht Deutsch` eine Form von Migrationsgeschichte deduzieren lässt und somit sprachliche Vielfalt als Indikator für kulturelle Vielfalt dient.

Kapelari, Suzanne (Hg.), Vierte „Tagung der Fachdidaktik“ 2019:

„Interdisziplinäre fachdidaktische Diskurse zur Bildung für nachhaltige Entwicklung“

(C) 2020 innsbruck university press, ISBN 978-3-99106-019-2, DOI 10.15203/99106-019-2 


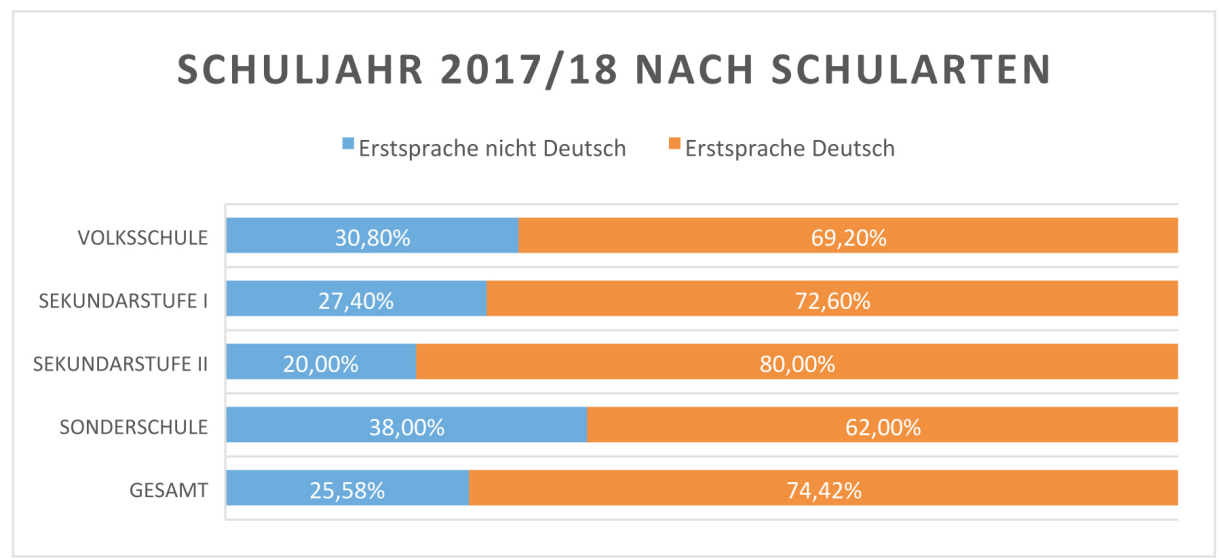

Abb. 1: Erstsprachen der Lernenden im Schuljahr 2017/18 nach Schularten (Eigene Darstellung J.P.)

Abbildung 1 verdeutlicht, dass im Schuljahr 2017/18 exakt ein Fünftel der SchülerInnen in der Sekundarstufe II angaben, eine nicht deutsche Erstsprache zu sprechen. Dieser Wert lag im Vergleich dazu in der Sekundarstufe I bei 27,4\%. Aufbauend auf diese Visualisierung bietet Abbildung 2 einen genaueren Einblick in die sprachlichen Gegebenheiten der SchülerInnen in der Sekundarstufe I, indem diese nach Schultypen aufgespaltet betrachtet wird. 


\section{SCHULJAHR 2017/18 SEKUNDARSTUFE I}

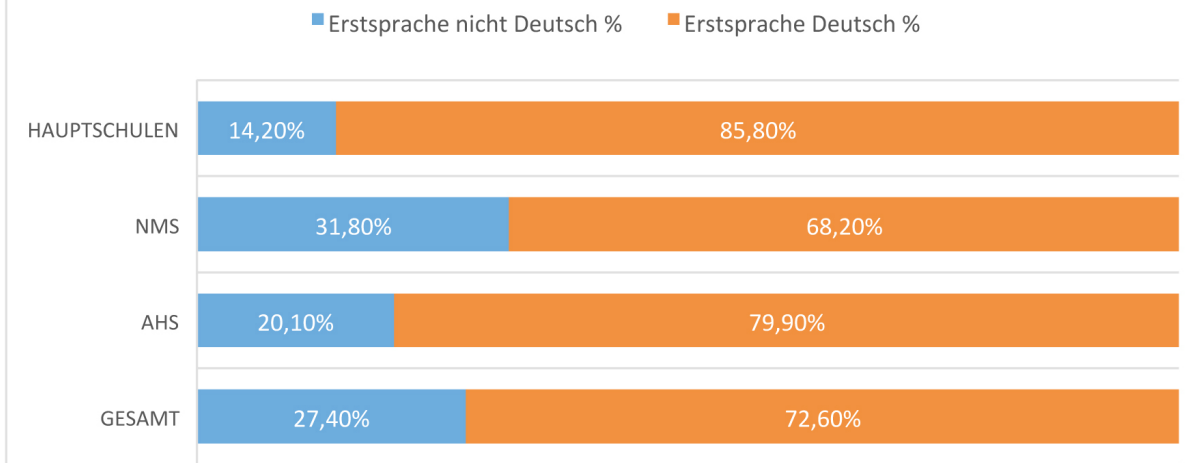

Abb. 2: Erstsprachen der Lernenden im Schuljahr 2017/18 in der Sekundarstufe I (Eigene Darstellung J.P.)

An Abbildung 2 lässt sich erkennen, dass im Schuljahr 2017/18 an Neuen Mittelschulen (NMS) der Anteil an Lernenden mit anderen Erstsprachen als Deutsch mit 31,8\% deutlich höher lag als an AHS-Schulen mit 20,1\% (Statistik Austria, 2018b). Abbildung 3 bietet nun einen 10-Jahres-Vergleich zum Anteil der Lernenden mit anderer Erstsprache als Deutsch, indem die Schuljahre 2017/18 und 2007/08 kontrastiert werden.

Es ist erkenntlich, dass im Schuljahr 2017/18 insgesamt mehr als ein Viertel aller Lernenden in Österreich angaben, eine andere Erstsprache als Deutsch zu sprechen. 10 Jahre zuvor lag dieser Prozentsatz erst bei rund 15,9\%, was einer Zunahme von über $60 \%$ entspricht. 


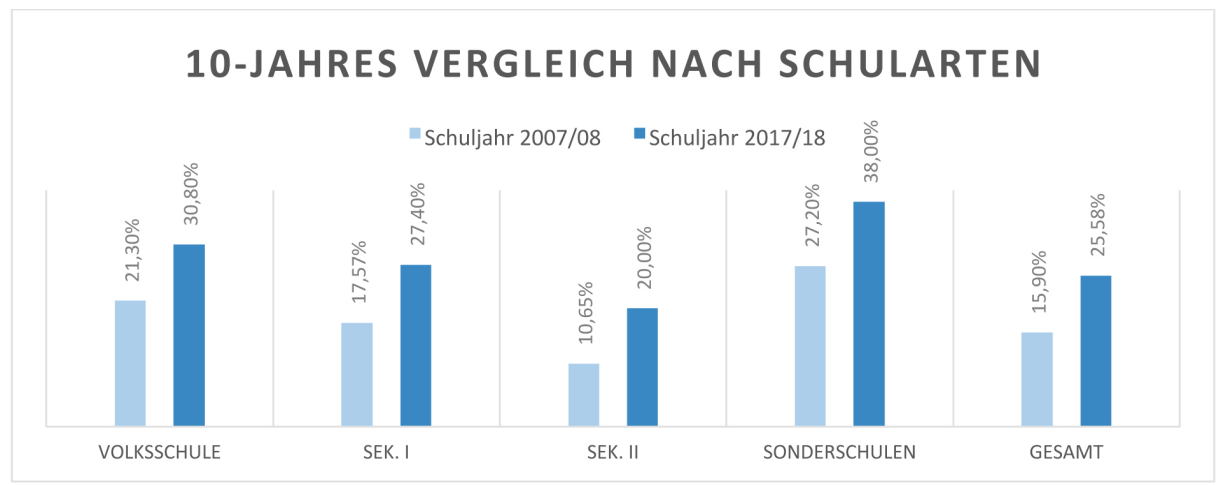

Abb. 3: Anteil Lernender mit Erstsprache nicht Deutsch im Schuljahr 2007/2008 und 2017/2018 (Eigene Darstellung J.P.)

\section{Forschungsstand und Desiderata}

In diesem Abschnitt soll nun der Stand der Forschung sowie Problemstellungen und Desiderata im Forschungsfeld der kulturellen Diversität im (Sprach-) Unterricht mit Fokus auf das österreichische Schulsystem beleuchtet werden.

Aufgrund der in Abschnitt 2 dargestellten Veränderungen in der SchülerInnenpopulation ergibt sich die Notwendigkeit, existierende Lehr- und Lernansätze entsprechend anzupassen und effektive Unterrichtsdesigns zu entwickeln, die die LernerInnen auf ein Leben in einer mehrsprachigen und mehrkulturellen Gesellschaft vorbereiten (Anton, 2017; Brunsmeier, 2016; Dausend, 2014; Delanoy, 2014a). Da Sprache und Kultur eng miteinander verzahnt sind (Freitag-Hild, 2018) und jeder Sprachunterricht gleichzeitig auch Kulturunterricht ist (Kramsch, 2006, S. 8), kommt der Sprachbildung in Bezug auf kulturelles Lernen eine besondere Rolle zu (Hu \& Byram, 2009). Da Sprache nicht nur Teil von Kultur ist, sondern diese gleichzeitig auch widerspiegelt und begründet, muss für erfolgreiches Kommunizieren Sprachverwendung eng mit kulturell angemessenem Verhalten gedacht werden (Blell \& Doff, 2014a, S. 78). Aufgrund ihrer Funktion als lingua franca, deren 
Reichweite historisch einzigartig ist (Baker, 2015, S. 9), hat die englische Sprache in diesem Zusammenhang einen speziellen Stellenwert, da sie die Grundlage des internationalen Austauschs zwischen Menschen unterschiedlicher kultureller Hintergründe darstellt (Brunsmeier, 2016, S. 13). Folglich wird das Einbeziehen einer Vielzahl an Kulturen in Lehr-Lernprozesse propagiert (Schlaak, 2015), da auf diese Weise die gesellschaftliche Realität im Klassenzimmer authentisch abgebildet und der Unterricht somit der zunehmenden sprachlichen und kulturellen Vielfalt der Lernenden gerecht werden kann (Burwitz-Melzer, 2017; Reimann, 2017; Schäfer, 2016, 2017a). In diesem Zusammenhang bekräftigt Doff (2016, S. 4), dass die Konzeption des Fremdsprachenunterrichts im Allgemeinen am stärksten durch sprachliche und kulturelle Heterogenität beeinflusst werden.

Als Folge dieser Entwicklungen und dem besonderen Stellenwert von Englisch als lingua franca in der interkulturellen Kommunikation (Baker, 2015) hat sich die Förderung der sogenannten interkulturellen (kommunikativen) Kompetenz (IKK) als übergeordnetes Ziel des Fremdsprachenunterrichts etabliert (Blell \& Doff, 2014b). Die Entwicklung von IKK wurde auch im grundlegenden Bildungsdokument des europäischen Sprachunterrichts, dem Gemeinsamen Europäischen Referenzrahmen für Sprachen (GERS), manifestiert (Council of Europe, 2001). Zudem stellt interkulturelles Lernen ein grundlegendes Bildungsziel und didaktisches Prinzip in den österreichischen Lehrplänen dar (BMBWK / Bundesministerium für Bildung, Wissenschaft und Kunst: Österreichischer Lehrplan AHS Unterstufe Lebende Fremdsprachen, 2006), dessen Bedeutsamkeit für schulische Lernprozesse in einem Grundsatzerlass im Jahr 2017 erneut bekräftigt wurde (BMB / Bundesministerium für Bildung: Interkulturelle Bildung: Grundsatzerlass, 2017).

Die für diesen Beitrag grundlegenden Begriffe des inter-, trans- und mehrkulturellen Lernens sollen nun skizziert werden. Vereinfacht dargestellt bezieht sich der Begriff interkulturell auf Gegebenheiten, in welchen Aspekte oder Menschen kulturell getrennt und verglichen werden und eine Gegenüberstellung zwischen „Selbst“ und „Anderem“ bzw. „Fremdem“

Kapelari, Suzanne (Hg.), Vierte „Tagung der Fachdidaktik“ 2019:

„Interdisziplinäre fachdidaktische Diskurse zur Bildung für nachhaltige Entwicklung“

(C) 2020 innsbruck university press, ISBN 978-3-99106-019-2, DOI 10.15203/99106-019-2 
stattfindet (Fäcke \& Meißner, 2019, S. 5). Im Sprachunterricht wird damit die Kompetenz verstanden, die Erstkultur mit der Zielkultur in Zusammenhang zu bringen und über Unterschiede zu reflektieren (Council of Europe, 2001, S. 104-105). Im Gegensatz dazu versucht das seit der Jahrtausendwende bestehende Konzept des transkulturellen Lernens (Welsch, 1999) diese Dichotomie aufzuheben und den Fokus auf vereinende, gemeinsame Aspekte sowie Hybridisierung zu legen (Fäcke \& Meißner, 2019, S. 5). Um kulturelle Lernprozesse im Sprachunterricht zu untersuchen entwickelte Michael Byram mit seinem Modell der IKK (Byram, 1997) eine Operationalisierung des interkulturellen Lernens. Obwohl das Modell bis heute als Grundlage zahlreicher Studien dient, haben konzeptuelle und terminologische Debatten zur Erweiterung bzw. Ablöse des Konstrukts des interkulturellen Lernens durch das transkulturelle Lernen zugenommen (Delanoy, 2014b; Freitag-Hild, 2018; Reimann, 2015). Folglich stellt der Begriff der Mehrkulturalität eine Klammer für inter- und transkulturelle Lernprozesse dar (Blell \& Doff, 2014b, S. 2) und setzt sich zum Ziel, kulturelle Hintergründe der SchülerInnen stärker in das Unterrichtsgeschehen miteinzubinden, wie es von zahlreichen AutorInnen gefordert wird (Bierwirth et al., 2017; Burwitz-Melzer, 2017; Doff, 2016; Reimann, 2017; Schlaak, 2015).

Neben einigen Handlungsempfehlungen zur Anbahnung von inter- und transkulturellen, demnach mehrkulturellen, Ansätzen im Sprachunterricht und trotz dessen mehrfach akzentuierter Bedeutung für Lernprozessen, lässt sich ein deutlicher Mangel an empirischer Forschung konstatieren (Anton, 2017; Brunsmeier, 2016; Schäfer, 2016; Trautmann, 2010). Eine der wenigen kürzlichen Forschungsarbeiten, die sich um die Schließung der Lücke an Empirie in Zusammenhang mit kulturellen Lernprozessen im Englischunterricht bemüht, sind Brunsmeier (2016) und Schäfer (2016). In ihrer Studie entwickelte Brunsmeier (2016) Aufgaben anhand von theoriegeleiteten Könnensbeschreibungen für den Englischunterricht der Primarstufe und erprobte empirisch, ob diese zur Förderung der interkulturellen kommunikativen Kompetenz der Lernenden beitragen. Im Englischunterricht der Sekundarstufe I beleuchtete Schäfer (2016) hingegen Möglichkeiten der Anbahnung kulturellen Lernens im

Kapelari, Suzanne (Hg.), Vierte „Tagung der Fachdidaktik“ 2019:

„Interdisziplinäre fachdidaktische Diskurse zur Bildung für nachhaltige Entwicklung“

(C) 2020 innsbruck university press, ISBN 978-3-99106-019-2, DOI 10.15203/99106-019-2 
Kontext heterogener Lerngruppen. Sie folgert aus ihrer design-based research Studie, dass die kulturelle Vielfalt der SchülerInnen über die Interaktion von und über verschiedene Sichtweisen und Deutungsmuster in den Englischunterricht miteinbezogen werden kann.

Neben diesen vereinzelten Forschungsarbeiten beklagt Doff (2016, S. 4) ein besonderes Defizit an Forschung im Englischunterricht der Sekundarstufe I. Des Weiteren konnte eine vorherrschende Unsicherheit der Lehrenden in Bezug auf den Umgang mit kultureller Vielfalt im Unterricht (Chilla \& Vogt, 2017) sowie bezüglich der Implementierung von inter- und transkulturellen Lernprozessen festgestellt werden (Brunsmeier, 2016). Obwohl das Bewusstsein der Lehrpersonen über das Bildungsziel der IKK nachgewiesen werden konnte (Brunsmeier, 2016, S. 90), führt diese Unsicherheit häufig zu einer Reduzierung der komplexen Konstrukte auf die Vermittlung von Landeskunde (Grünewald, 2012, S. 55),

Schließlich wird die Notwendigkeit deutlich, geeignete Aufgabenstellungen für den Sprachunterricht zu entwickeln, deren Potenzial zur Anbahnung mehrkultureller Lernprozesse zu untersuchen und Implikationen für schulische Lehr-Lernprozesse abzuleiten, durch die Lehrpersonen in der Implementierung des Konstrukts unterstützt werden können (Anton, 2017; Bär, 2017; Brunsmeier, 2016; Göbel, Lewandowska, \& Diehr, 2017; Schäfer, 2017b).

\section{Mehrkulturalität und Bildung für nachhaltige Entwicklung}

In diesem Abschnitt soll der Zusammenhang sowie mögliche Anknüpfungspunkte zwischen dem Forschungsfeld der kulturellen Vielfalt im (Sprach)Unterricht sowie der Bildung für nachhaltige Entwicklung herausgearbeitet werden.

In Bezug auf kulturelle Vielfalt und Bildungserfolg hält die Erziehungswissenschaftlerin Ingrid Gogolin (2016, S. 62) fest, dass die Forschung zeigt, dass

Kapelari, Suzanne (Hg.), Vierte „Tagung der Fachdidaktik“ 2019:

„Interdisziplinäre fachdidaktische Diskurse zur Bildung für nachhaltige Entwicklung“

(C) 2020 innsbruck university press, ISBN 978-3-99106-019-2, DOI 10.15203/99106-019-2 
Lernende mit Migrationshintergrund die Schullaufbahn durchschnittlich deutlich weniger erfolgreich durchlaufen als Gleichaltrige ohne Migrationshintergrund. Sie kritisiert zudem den Mangel an empirischer Forschung, die die Bedeutung der Unterrichtsgestaltung zum potenziellen Entgegenwirken dieser Differenzen untersucht. Diesbezüglich wird von Bierwirth, Blell und Fuchs argumentiert:

„Das Primat, allen Schüler*innen gleiche Chancen auf Bildung bzw. eine maximale Bildungsteilhabe zu gewähren, muss stärker in den Blick schulischen Alltags und sämtlicher Reformbemühungen im Bildungsbereich genommen werden. Eine Pädagogik der Vielfalt, oder auch der sogenannte diversity-Ansatz (Anerkennung der Vielfalt), wird auf vielen gesellschaftlichen Ebenen diskutiert - darunter auch der Bildungsbereich und sollte ein wesentliches Merkmal unserer Gesellschaft sein.“ (Bierwirth et al., 2017)

Diese Forderung nach Chancengleichheit in der Bildung findet sich auch in den von den Vereinten Nationen im Jahr 2015 formulierten politischen Zielen für nachhaltige Entwicklung, den so genannten Sustainable Development Goals (SDGs, siehe Rieckmann in diesem Band), wieder. Der Bildungsbereich soll hierbei bestmöglich einen Beitrag zu jedem formulierten Ziel leisten (United Nations, 2019a).

Schnittstellen zwischen den SDGs und dem Forschungskontext der kulturellen Vielfalt im (Sprach-)Unterricht sind der Bereich 4 (Quality Education) sowie SDG 10 (Reduced Inequalities). Letzteres beschreibt, dass es grundsätzlich universeller politischer Bestimmungen bedarf, um verstärkt auf die Bedürfnisse der benachteiligten und marginalisierten Bevölkerung $\mathrm{zu}$ achten (United Nations, 2019b). Weiters führt der Bereich Nummer 4, der sich konkret auf den Bildungsbereich bezieht, an, dass eine Verbesserung der Bildungsgegebenheiten durch eine adäquate Ausbildung der Lehrkräfte, vor allem im Umgang mit Minderheiten und Heterogenität, erreicht werden kann (United Nations, 2019c). Die internationale Bildungskampagne ,Bildung für nachhaltige Entwicklung hält folglich fest: 
„Kulturkonflikte treten in verschiedenen Gesellschaftsbereichen auf, so auch in Kontexten der Schule. Die Bereitschaft zum Dialog ist eine wichtige Voraussetzung, um den Mehrwert kultureller Diversität erfahren zu können. In Schulen bietet sich insbesondere die Gelegenheit - etwa durch Gruppenarbeit im Besonderen und den Klassenverband im Allgemeinen soziale Kompetenzen zu erlernen.“(UNESCO-Weltaktionsprogramm, 2019)

In ähnlicher Formulierung findet sich die obige Aussage auch in dem (Sprach-) Unterricht zugrunde liegenden, bildungspolitischen Dokumenten wie dem GERS (Council of Europe, 2001, 2018) oder den österreichischen Lehrplänen wieder. In den Schulcurricula ist interkulturelles Lernen als ein Bildungsprinzip verankert (BMB / Bundesministerium für Bildung: Interkulturelle Bildung: Grundsatzerlass, 2017) und $\mathrm{zu}$ vermittelnde Werte wie Gleichberechtigung, Offenheit und Respekt werden besonders hervorgehoben (BMBWK / Bundesministerium für Bildung, Wissenschaft und Kunst: Österreichischer Lehrplan AHS Unterstufe Lebende Fremdsprachen, 2006). Somit wird deutlich, dass eine erhöhte Chancengleichheit im Bildungserfolg (SDG 10) und eine gesteigerte Qualität des Lehrens und Lernens (SDG 4) vor allem durch eine verstärkte Schulung der Lehrpersonen, speziell in Hinblick auf den effizienten und sensiblen Umgang mit kultureller Vielfalt, erreicht werden kann.

Es ist deshalb essentiell zu erkennen, dass Bildung niemals als kulturneutral gedacht werden darf, Lernprozesse nicht in einem Vakuum stattfinden und weder Lehrende noch Lernende frei von kulturellen Einflüssen sind. Es muss Ziel nachhaltiger Bildung sein, ein Bewusstsein und eine Wertschätzung für kulturelle Vielfalt zu schaffen und dadurch Lernende in der Entwicklung ihrer kulturell verankerten Fähigkeiten zu unterstützen (UNESCO, 2009, S. 97).

Eine weitere Schnittstelle zwischen Bildung für nachhaltige Entwicklung und Mehrkulturalität im (Sprach-)Unterricht stellt der Kompetenzbegriff dar. Wie in Abschnitt 3 erläutert bietet Byrams 5-dimensionales Modell der interkulturellen kommunikativen Kompetenz (Byram, 1997), welche die Fähigkeit meint, „relevante Einstellungen, Fertigkeiten, Wissen und kritisches Verständnis zu nutzen, um angemessen auf Herausforderungen und Gelegenheiten in der

Kapelari, Suzanne (Hg.), Vierte „Tagung der Fachdidaktik“ 2019:

„Interdisziplinäre fachdidaktische Diskurse zur Bildung für nachhaltige Entwicklung“

(C) 2020 innsbruck university press, ISBN 978-3-99106-019-2, DOI 10.15203/99106-019-2 
Interaktion mit Vertretern einer anderen kulturellen Gruppe zu reagieren“ (Byram, 2019, S. 252), eine Grundlage für Forschungsarbeiten in der Sprachendidaktik. Auf dem Gebiet der Nachhaltigkeitsforschung wird in ähnlicher Form die so genannte interpersonal competence als eine von fünf definierten Kernkompetenzen von sustainability genannt. Interpersonal competence beschreibt die Fähigkeit, die Vielfalt an Kulturen, sozialen Gruppen, Gemeinschaften und Individuen zu verstehen, aufzugreifen und $\mathrm{zu}$ fördern. Da für diesen Kompetenzbereich Aspekte wie pluralistisches und transkulturelles Denken relevant sind (Wiek, Withycombe, \& Redman, 2011, S. 211), ergeben sich hier Synergien zwischen dem Forschungsfeld der Mehrkulturalität und dem der Nachhaltigkeit, deren Potenzial in künftigen Studien vermehrt ausgeschöpft werden kann. An dieser Stelle ist positiv zu vermerken, dass der Begriff des transkulturellen Lernens bereits Einzug in den Kompetenzdiskurs findet.

In Bezug auf Bildungsgerechtigkeit und Mehrkulturalität plädiert Doff (2016, S. 4) für die Generierung von sowohl fachspezifischen als auch interdisziplinär ausgerichteten, empirisch überprüften Methoden und Ansätzen, die das Potenzial der lebensweltlichen Mehrkulturalität der Lernenden in der Schule nutzen und aktiv in das Unterrichtsgeschehen einbinden. Gogolin (2016, S. 64) vermerkt diesbezüglich in letzter Zeit eine Zunahme der Forschungsaktivität in Hinblick auf die Gestaltung von Lehr-Lernprozessen im Bildungsbereich zur Nutzung der Chancen von sprachlicher und kultureller Heterogenität für das schulische Lernen.

\section{Forschungsprojekt}

In Anbetracht der zunehmenden kulturellen Vielfalt im österreichischen Bildungssystem (Statistik Austria, 2018b) und des bestehenden Mangels an Forschung und empirischen Daten zur Implementierung von mehrkulturellen Lernprozessen setzt sich die Forschungsarbeit zum Ziel, an einige der aufgezeigten Desiderate anzuknüpfen. Folglich ist eine empirische Studie in 
Planung, in welcher ein theoriebasiertes und praxisrelevantes methodisches Design für den Englischunterricht entwickelt, implementiert und empirisch evaluiert wird. Dieses Unterrichtsdesign bezieht im Sinne der Didaktik der Mehrkulturalität (Reimann, 2017) die kulturellen Gegebenheiten der Lernenden mit ein und leistet somit einen Beitrag in der Anbahnung von SDG 4 und 10 (United Nations, 2019a). Die Studie ist in der österreichischen Sekundarstufe I, konkret in Englischklassen der Neuen Mittelschule (NMS), verortet, da in diesem Schultyp das Ausmaß an sprachlicher und somit kultureller Vielfalt der Lernenden im Schuljahr 2017/18 besonders hoch war (siehe Abschnitt 2).

Die folgenden Fragestellungen sind für die Forschungsarbeit wegweisend:

Forschungsfrage 1: Wie werden mehrkulturelle Lernprozesse von Lehrpersonen im Englischunterricht der österreichischen Sekundarstufe I angebahnt?

Forschungsfrage 2: Welche Herausforderungen entstehen in Verbindung mit der Anbahnung von mehrkulturellen Lernprozessen im Englischunterricht der österreichischen Sekundarstufe I?

Forschungsfrage 3: Auf welche Art und in welchem Umfang werden mehrkulturelle Lernprozesse in den Aufgabenstellungen der Lehrwerke für den Englischunterricht der österreichischen Sekundarstufe I angebahnt?

Forschungsfrage 4: Welche Dimensionen des mehrkulturellen Lernens können durch Aufgabenorientierung im Englischunterricht der österreichischen Sekundarstufe I angebahnt werden?

Abbildung 4 visualisiert die Struktur des Forschungsprojekts, das durch Anwendung von sowohl qualitativen als auch quantitativen Elementen als

Kapelari, Suzanne (Hg.), Vierte „Tagung der Fachdidaktik“ 2019:

„Interdisziplinäre fachdidaktische Diskurse zur Bildung für nachhaltige Entwicklung“

(C) 2020 innsbruck university press, ISBN 978-3-99106-019-2, DOI 10.15203/99106-019-2 
mixed-methods Design (Kuckartz, 2014) konzipiert ist. Pro Abschnitt gibt die Abbildung zudem Aufschluss über die jeweilige Erhebungsart und das Sample und zeigt die Verbindung zu den obigen Forschungsfragen.

\section{TeIL 1: Bedarfsanalyse \& STATUS-Quo ERHEbung}

\subsection{ONLINE BEFRAGUNG (FORSCHUNGSFRAGE 1, 2)}

$\checkmark$ Quantitativ

$\checkmark$ Sample: 100 TeilnehmerInnen; Englisch Lehrpersonen in der österreichischen

Sekundarstufe I

\subsection{LEHRWERKSANALYSE (FORSCHUNGSFRAGE 3)}

$\checkmark$ Qualitativ \& Quantitativ

$\checkmark$ Korpus: 12 Englisch Lehrwerke für die österreichische Sekundarstufe I

(student's books, Arbeitsbücher und Lehrerhandbücher)

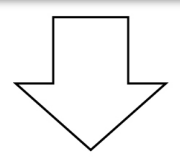

TEIL 2: DESIGN-BASED RESEARCH STUDIE (DBR)

Iterativ: Design, Versuch, Evaluierung, Re-design (FORSCHUNGSFRAGE 4)

\subsection{DBR-ZYKLUS I}

$\checkmark$ Qualitativ \& Quantitativ

$\checkmark$ Sample: Zwei Englischklassen der neuen Mittelschule (NMS), vier Lehrpersonen, ca. 35 Lernende

\subsection{DBR-ZYKLUS II}

$\checkmark$ Qualitativ \& Quantitativ

$\checkmark$ Sample: Zwei Englischklassen der neuen Mittelschule (NMS), vier Lehrpersonen, ca. 35 Lernende

Abb. 4: Forschungsdesign (Eigene Darstellung J.P.) 
Wie in Abbildung 4 ersichtlich ist, wird im ersten Teil der Studie eine StatusQuo Erhebung zum Status und zur Umsetzung des Bildungsziels des interkulturellen Lernens im Englischunterricht der Sekundarstufe I durchgeführt. Anhand dieser sollen Herausforderungen und Förderbedarf in Zusammenhang mit der Anbahnung von inter-, trans- und somit mehrkulturellen Lernprozessen aufgezeigt werden. Im anschließenden zweiten Teil der Forschungsarbeit wird eine design-based research Studie (DBR) (Euler, 2014) durchgeführt. Diese setzt sich zum Ziel, ein theoriebasiertes, praxisrelevantes Unterrichtsdesign nach Vorbild des aufgabenorientierten Sprachunterrichts iterativ zu entwickeln, zu erproben und schließlich zu evaluieren, inwieweit mehrkulturelle Lernprozesse im Englischunterricht der Sekundarstufe I angebahnt werden können. Durch das dabei entstehende, empirisch fundierte Unterrichtsmodell wird ein erster methodischer Rahmen zur Anbahnung von mehrkulturellen Lernprozessen im Englischunterricht geschaffen, der eine wertvolle Unterstützung für Lehrpersonen im Umgang mit kultureller Vielfalt im Unterricht darstellen kann.

\section{Zusammenfassung und Ausblick}

In diesem Beitrag wurde ein Forschungsprojekt vorgestellt, welches durch die Anbahnung von mehrkulturellen Lernprozessen im Englischunterricht der österreichischen Sekundarstufe I versucht, die Chancen der zunehmenden kulturellen Vielfalt der Lernenden aktiv in das Unterrichtsgeschehen miteinzubinden. Durch das entwickelte Unterrichtsdesign können Lehrpersonen sowohl in der Umsetzung von verankerten Bildungszielen als auch im Umgang mit kultureller Diversität im Klassenzimmer unterstützt werden. Es wurde erläutert, dass hier Überschneidungen mit den Grundanliegen der Bildung für Nachhaltige Entwicklung sowie den Sustainable Development Goals vorliegen. Ebenso zeigen sich in verwendeten Kompetenzmodellen der Sprach- sowie der Nachhaltigkeitsforschung Synergien und mögliche Ausrichtungen für künftige Forschungsarbeiten.

Kapelari, Suzanne (Hg.), Vierte ,Tagung der Fachdidaktik“ 2019:

„Interdisziplinäre fachdidaktische Diskurse zur Bildung für nachhaltige Entwicklung“

(C) 2020 innsbruck university press, ISBN 978-3-99106-019-2, DOI 10.15203/99106-019-2 
Abschließend ist festzuhalten, dass sprachliche und kulturelle Diversität in Gesellschaft und Bildung schon längst kein Novum mehr darstellen und aufgrund von Globalisierung und Migrationsbewegungen weiter zunehmen werden (Gogolin, 2016). Es ist deshalb von entscheidender Bedeutung, auf diese sich verändernden Gegebenheiten auch im Bildungskontext zu reagieren und folglich bestehende Unterrichtsansätze und -methoden zu überdenken und entsprechend zu adaptieren. Eine zeitgemäße Pädagogik und Didaktik muss sich zunehmend in Bezug auf mehrkulturelles Lernen reflektieren und hinterfragen, ob möglichst alle Lernenden von den Unterrichtsinhalten und -methoden angesprochen werden. Die kulturelle Vielfalt wird jedoch im Unterricht vermehrt als Herausforderung anstatt als Chance für das Lehren und Lernen wahrgenommen. Forschungsarbeiten könnten anhand entsprechender Erkenntnisse die Bedeutsamkeit der Thematik hervorheben und Lehrpersonen in Bezug auf den effektiven Umgang mit kultureller Vielfalt sensibilisieren. Die vorgestellte Studie stellt eine Möglichkeit dar, anhand von mehrkulturellen Lernprozessen der kulturellen Vielfalt der Lernenden gerecht zu werden und gleichzeitig alle SchülerInnen auf ein Leben in einer mehrsprachigen und mehrkulturellen Gesellschaft vorzubereiten.

\section{Literatur}

Anton, D. (2017). Inter- und transkulturelles Lernen im Englischunterricht: Eine didaktische Analyse einschlägiger Lehrbücher. Heidelberg: Universitätsverlag Winter.

Baker, W. (2015). Culture and identity through English as a Lingua Franca: Rethinking concepts and goals in intercultural communication. Developments in English as a Lingua Franca: Vol. 8. Berlin, Boston: De Gruyter Mouton. https://doi.org/10.1515/9781501502149

Bär, M. (2017). Auf dem Weg zur inklusiven Schule: Mögliche Implikationen aus fremdsprachendidaktischer Perspektive. In E. Burwitz-Melzer, F. G. Königs, C. Riemer, \& L. Schmelter (Eds.), Giessener Beiträge zur Fremdsprachendidaktik. Inklusion, Diversität und das Lehren und Lernen fremder Sprachen: Arbeitspapiere der 37. Frühjahrskonferenz zur Erforschung des Fremdsprachenunterrichts (pp. 10-20). Tübingen: Narr Francke Attempto.

Kapelari, Suzanne (Hg.), Vierte „Tagung der Fachdidaktik“ 2019:

„Interdisziplinäre fachdidaktische Diskurse zur Bildung für nachhaltige Entwicklung“

(C) 2020 innsbruck university press, ISBN 978-3-99106-019-2, DOI 10.15203/99106-019-2 
Bierwirth, A., Blell, G., \& Fuchs, S. (2017). Wie divers ist Englischlernen?: Konzepte in Forschung und Praxis zum inklusiven Englischunterricht. Zeitschrift Für Inklusion. (3). Retrieved from https://www.inklusion-online.net/index.php/inklusion-online/article/view/447

Blell, G., \& Doff, S. (2014a). It takes more than two for this tango: Moving beyond the self/other-binary in teaching about culture in the global EFL-classroom. Zeitschrift Für Interkulturellen Fremdsprachenunterricht, 19(1), 77-96.

Blell, G., \& Doff, S. (2014b). Mehrsprachigkeit und Mehrkulturalität: Einführung in das Thema. Zeitschrift Für Interkulturellen Fremdsprachenunterricht, 19(1), 1-7.

BMB / Bundesministerium für Bildung: Interkulturelle Bildung: Grundsatzerlass (2017).

BMBWK / Bundesministerium für Bildung, Wissenschaft und Kunst: Österreichischer Lehrplan AHS Unterstufe Lebende Fremdsprachen (2006).

Brunsmeier, S. (2016). Interkulturelle Kommunikative Kompetenz im Englischunterricht der Grundschule: Grundlagen, Erfahrungen, Perspektiven. Tübingen: Narr.

Burwitz-Melzer, E. (2017),Same same but different': Inklusion, Heterogenität und Diversität im Englischunterricht. In E. Burwitz-Melzer, F. G. Königs, C. Riemer, \& L. Schmelter (Eds.), Giessener Beiträge zur Fremdsprachendidaktik. Inklusion, Diversität und das Lehren und Lernen fremder Sprachen: Arbeitspapiere der 37. Frühjahrskonferenz zur Erforschung des Fremdsprachenunterrichts (pp. 31-42). Tübingen: Narr Francke Attempto.

Byram, M. (1997). Teaching and Assessing Intercultural Communicative Competence. Clevedon: Multilingual Matters.

Byram, M. (2019). Evaluation/Assessment und Selbstevaluation/Assessment interkultureller Kompetenzen. In C. Fäcke \& F.-J. Meißner (Eds.), Handbuch Mehrsprachigkeits- und Mehrkulturalitätsdidaktik (pp. 251-256).

Chilla, S., \& Vogt, K. (2017). Einleitung. In S. Chilla \& K. Vogt (Eds.), Heterogenität und Diversität im Englischunterricht: Fachdidaktische Perspektiven (pp.712). Frankfurt am Main: Peter Lang.

Council of Europe (2001). Common European framework of reference for languages: Learning, teaching, assessment. Retrieved from https://rm.coe.int/1680459f97

Council of Europe (2018). Common European framework of reference for languages. Learning, teaching, assessment: Companion volume with new descriptors. Retrieved from https://rm.coe.int/1680787989

Dausend, H. (2014). Mit Street Art transkulturelle Lernprozesse intiieren. In F. Matz, M. Rogge, \& S. Siepmann (Eds.), Transkulturelles Lernen im Fremdsprachenunterricht: Theorie und Praxis (1st ed., pp. 89-100). Frankfurt a.M: Peter Lang $\mathrm{GmbH}$ Internationaler Verlag der Wissenschaften.

Delanoy, W. (2014a). Mehrsprachigkeit, Englisch und Literatur(unterricht). Zeitschrift Für Interkulturellen Fremdsprachenunterricht, 19(1), 63-76.

Kapelari, Suzanne (Hg.), Vierte „Tagung der Fachdidaktik“ 2019:

„Interdisziplinäre fachdidaktische Diskurse zur Bildung für nachhaltige Entwicklung“

(C) 2020 innsbruck university press, ISBN 978-3-99106-019-2, DOI 10.15203/99106-019-2 
Delanoy, W. (2014b). Transkulturalität als begriffliche und konzeptuelle Herausforderung an die Fremdsprachendidaktik. In F. Matz, M. Rogge, \& S. Siepmann (Eds.), Transkulturelles Lernen im Fremdsprachenunterricht: Theorie und Praxis (1st ed., pp. 19-35). Frankfurt a.M: Peter Lang GmbH Internationaler Verlag der Wissenschaften.

Doff, S. (2016). Heterogenität im Fremdsprachenuntericht: Kontext - Aufbau und Inhalt - Ausgangs- und Kristallisationsounkte. In S. Doff (Ed.), Heterogenität im Fremdsprachenunterricht: Impulse-Rahmenbedingungen-Kernfragen-Perspektiven (pp. 1-5). Tübingen: Narr.

Euler, D. (2014). Design Research - a paradigm under development. In D. Euler \& S. F.E. Sloane (Eds.), Design-Based Research (pp. 15-44). Stuttgart: Franz Steiner Verlag.

Fäcke, C., \& Meißner, F. J. (2019). Einleitung. In C. Fäcke \& F.-J. Meißner (Eds.), Handbuch Mehrsprachigkeits- und Mehrkulturalitätsdidaktik (pp. 1-16).

Freitag-Hild, B. (2018). Teaching Culture - Intercultural Competence, Transcultural Learning, Global Education. In C. Surkamp \& B. Viebrock (Eds.), Teaching English as a Foreign Language: An Introduction (pp. 159-175). Stuttgart: J.B. Metzler.

Göbel, K., Lewandowska, Z. M.， \& Diehr, B. (2017). Lernziel interkulturelle Kompetenz - Lernangebote im Englischunterricht der Klassenstufe 9 - Eine Reanalyse der Unterrichtsvideos der DESI-Studie. Zeitschrift Für Interkulturellen Fremdsprachenunterricht, 22(1), 107-121.

Gogolin, I. (2016). Folgen der Migration für Bildung und Erziehung. In E. BurwitzMelzer, G. Mehlhorn, C. Riemer, K.-R. Bausch, \& H.-J. Krumm (Eds.), UTB: Vol. 8043. Handbuch Fremdsprachenunterricht (6th ed., pp.60-64). Tübingen: A. Francke Verlag.

Grünewald, A. (2012). Förderung interkultureller Kompetenz durch Lernaufgaben. Fremdsprachen Lehren Und Lernen, 41(1), 54-71.

Hu, A., \& Byram, M. (2009). Introduction. In A. Hu \& M. Byram (Eds.), Interkulturelle Kompetenz und fremdsprachliches Lernen: Modelle, Empirie, Evaluation = Intercultural competence and foreign language learning; models, empiricism, assessment (pp. VII-XXV). Tübingen: Gunter Narr Verlag.

Kramsch, C. (2006). Context and culture in language teaching ([Nachdr.]). Oxford applied linguistics. Oxford: Oxford Univ. Press.

Kuckartz, U. (2014). Mixed Methods: Methodologie, Forschungsdesigns und Analyseverfahren. Wiesbaden: Springer VS. https://doi.org/10.1007/978-3-531-93267-5 
Lo Bianco, J. (2009). Dilemmas of efficiency, identity and worldmindedness. In J. Miller, A. Kostogriz, \& M. Gearon (Eds.), New perspectives on language and education. Culturally and linguistically diverse classrooms: new dilemmas for teachers (pp. 113-131). Bristol, UK: Multilingual Matters.

Reimann, D. (2015). Inter- und transkulturelle kommunikative Kompetenz.

Reimann, D. (2017). Interkulturelle Kompetenz. Narr Starter. Tübingen: Narr Francke Attempto.

Schäfer, L. (2016). Wie kann kulturelles Lernen im Englischunterricht der Sekundarstufe I in heterogenen Lerngruppen angebahnt werden?: Erste Ergebnisse einer Design-Based Research-Studie. In S. Doff (Ed.), Heterogenität im Fremdsprachenunterricht: Impulse-Rahmenbedingungen-Kernfragen-Perspektiven (pp. 91-106). Tübingen: Narr.

Schäfer, L. (2017a). Förderung kulturell-visueller Kompetenzen mit Street Art im Englischunterricht. In S. Doff \& R. Komoss (Eds.), Making Change Happen: Wandel im Fachunterricht analysieren und gestalten (pp. 69-74). Wiesbaden: Springer VS.

Schäfer, L. (2017b). Von bunten Wänden und blumenwerfenden Demonstranten - Die Design-basierte Entwicklung und Erprobung einer Unterrichtssequenz zu Street Art im inklusiven Englischunterricht der Sekundarstufe I. In C. Schlaak \& S. Thiele (Eds.), Romanische Sprachen und ihre Didaktik: Band 62. Migration, Mehrsprachigkeit und Inklusion: Strategien für den schulischen Unterricht und die Hochschullehre (pp. 141-157). Stuttgart: ibidem-Verlag.

Schlaak, C. (2015). Fremdsprachendidaktik und Inklusionspädagogik: Herausforderungen im Kontext von Migration und Mehrsprachigkeit. Stuttgart: ibidem.

Statistik Austria (2018a). Bevölkerung 1951 bis 2016 nach demographischen Merkmalen. Retrieved from https://www.statistik.at/web de/statistiken/menschen und gesellschaft/bevoel kerung/volkszaehlungen registerzaehlungen abgestimmte erwerbsstatistik/be voelkerung nach demographischen merkmalen/078573.html

Statistik Austria (2018b). Schülerinnen und Schüler mit nicht-deutscher Umgangssprache im Schuljahr 2017/18. Retrieved from https://www.statistik.at/web de/statistiken/menschen und gesellschaft/bildung und kultur/formales bildungswesen/schulen schulbesuch/index.html

Statistik Austria (2019). Bevölkerung mit Migrationshintergrund seit 2008. Retrieved from https:/www.statistik.at/web de/statistiken/menschen und gesellschaft/bevoel kerung/bevoelkerungsstruktur/bevoelkerung_nach_migrationshintergrund/0694 43.html

Sturm, T. (2016). Lehrbuch Heterogenität in der Schule (2nd ed.). München: Ernst Reinhardt Verlag.

Kapelari, Suzanne (Hg.), Vierte „Tagung der Fachdidaktik“ 2019:

„Interdisziplinäre fachdidaktische Diskurse zur Bildung für nachhaltige Entwicklung“

(C) 2020 innsbruck university press, ISBN 978-3-99106-019-2, DOI 10.15203/99106-019-2 
Trautmann, M. (2010). Heterogenität. (k)ein Thema der Fremdsprachendidaktik? In A. Köker, S. Romahn, \& A. Textor (Eds.), Herausforderung Heterogenität: Ansätze und Weichenstellungen (pp. 52-64). Bad Heilbrunn: Klinkhardt.

UNESCO (2009). UNESCO world report: Investing in cultural diversity and intercultural dialogue. World reports series. Paris: Unesco.

UNESCO-Weltaktionsprogramm (2019). Bildung für nachhaltige Entwicklung: Bildung für nachhaltige Entwicklung, Inklusion, Migration und Demokratie-Lernen. Retrieved from https://www.bne-portal.de/de/einstieg/bildungsbereiche/schule\#

United Nations (2019a). Sustainable Development Goals. Retrieved from https://www.un.org/sustainabledevelopment/sustainable-development-goals/

United Nations (2019b). Sustainable Development Goals: Goal 10: Reduce inequality within and among countries. Retrieved from https://www.un.org/sustainabledevelopment/inequality/

United Nations (2019c). Sustainable Development Goals: Goal 4: Quality Education. Retrieved from https://www.un.org/sustainabledevelopment/education/

Welsch, W. (1999). Transculturality: The Puzzling form of Cultures Today. In M. Featherstone \& S. Lash (Eds.), Theory, culture \& society. Spaces of culture: City, nation, world (pp. 195-213). London, Thousand Oaks, Calif: Sage. https://doi.org/10.4135/9781446218723.n11

Wiek, A., Withycombe, L., \& Redman, C. L. (2011). Key competencies in sustainability: a reference framework for academic program development. Integrated Research System for Sustainability Science, 6(2), 203-218. https://doi.org/10.1007/s11625-011-0132-6 
Der vorliegende Band umfasst Forschungsbeiträge, die im Rahmen der "Vierten Tagung der Fachdidaktik: Interdisziplinäre fachdidaktische Diskurse zur Bildung für nachhaltige Entwicklung" an der Leopold-Franzens-Universität Innsbruck und der Pädagogischen Hochschule Tirol im Mai 2019 präsentiert wurden. Er dokumentiert die breite Fülle an Ansätzen, Fragestellungen und Methoden, mit der sich fachdidaktische Forschung am Hochschulstandort Innsbruck beschäftigt. Die vierte Tagung der Fachdidaktik diente dem interdisziplinären Diskurs und enthält neben der Verschriftlichung des einleitenden Plenarvortrages sieben Vorträge, die beispielgebend aufzeigen, welche Beiträge die einzelnen Unterrichtsfächer zum Bildungskanon einer nachhaltigen Entwicklung leisten können.

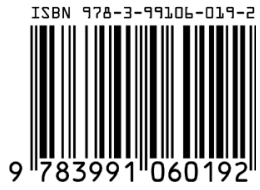

